Issued by Sandia National Laboratories, operated for the United States Department of Energy by Sandia Corporation.

NOTICE: This report was prepared as an account of work sponsored by an agency of the United States Government. Neither the United States Government nor any agency thereof, nor any of their employees, nor any of their contractors, subcontractors, or their employees, makes any warranty, express or implied, or assumes any legal liability or responsibility for the accuracy, completeness, or usefulness of any information, apparatus, product or process disclosed, or represents that its use would not infringe privately owned rights. Reference herein to any specific commercial product, process, or service by trade name, trademark, manufacturer, or otherwise, does not necessarily constitute or imply its endorsement, recommendation, or favoring by the United States Government, any agency thereof or any of their contractors or subcontractors. The views and opinions expressed herein do not necessarily state or reflect those of the United States Government, any agency thereof or any of their contractors.

Printed in the United States of America

Available from

National Technical Information Service

U.S. Department of Commerce

5285 Port Royal Road

Springfield, VA 22161

NTIS price codes

Printed copy: A07

Microfiche copy: A01 


\section{DISCLAIMER}

This report was prepared as an account of work sponsored by an agency of the United States Government. Neither the United States Government nor any agency Thereof, nor any of their employees, makes any warranty, express or implied, or assumes any legal liability or responsibility for the accuracy, completeness, or usefulness of any information, apparatus, product, or process disclosed, or represents that its use would not infringe privately owned rights. Reference herein to any specific commercial product, process, or service by trade name, trademark, manufacturer, or otherwise does not necessarily constitute or imply its endorsement, recommendation, or favoring by the United States Government or any agency thereof. The views and opinions of authors expressed herein do not necessarily state or reflect those of the United States Government or any agency thereof. 


\section{DISCLAIMER}

Portions of this document may be illegible in electronic image products. Images are produced from the best available original document. 
SAND87-0070

Unlimited Release

Printed August 1988
Distribution

Categorv UC 700

SAND $-87-0070$

DE88 015366

\title{
Analytical Approximations for X-Ray Cross Sections III
}

\author{
Frank Biggs \\ Test Planning and Diagnostics Division \\ Ruth Lighthill \\ Radiation Applications Division \\ Sandia National Laboratories \\ Albuquerque, NM 87185
}

\begin{abstract}
This report updates our previous work that provided analytical approximations to cross sections for both photoelectric absorption of photons by atoms and incoherent scattering of photons by atoms. This representation is convenient for use in programmable calculators and in computer programs to evaluate these cross sections numerically. The results apply to atoms of atomic numbers between 1 and 100 and for photon energies $\geq 10 \mathrm{eV}$. The photoelectric cross sections are again approximated by four-term polynomials in reciprocal powers of the photon energy. There are now more fitting intervals, however, than were used previously. The incoherent-scattering cross sections are based on the Klein-Nishina relation, but use simpler approximate equations for efficient computer evaluation. We describe the averaging scheme for applying these atomic results to any composite material. The fitting coefficients are included in tables, and the cross sections are shown graphically.
\end{abstract}

\section{DISCLAIMER}

This report was prepared as an account of work sponsored by an agency of the United States Government. Neither the United States Government nor any agency thereof, nor any of their employees, makes any warranty, express or implied, or assumes any legal liability or responsibility for the accuracy, completeness, or usefulness of any information, apparatus, product, or process disclosed, or represents that its use would not infringe privately owned rights. Reference herein to any specific commercial product, process, or service by trade name, trademark, manufacturer, or otherwise does not necessarily constitute or imply its endorsement, recommendation, or favoring by the United States Government or any agency thereof. The views and opinions of authors expressed herein do not necessarily state or reflect those of the United States Government or any agency thereof.

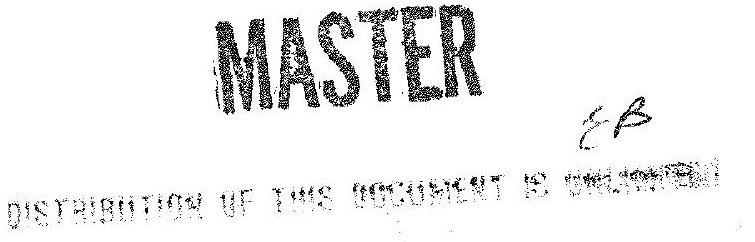




\section{Acknowledgments}

We take this opportunity to thank all the people who have given us helpful suggestions over the years. This will be the last revision by this combination of authors because Ruth Lighthill has retired. Frank Biggs joins the rest of the user community in thanking Ruth for her valuable efforts in this activity and in wishing her a happy retirement. 


\section{Preface}

The authors have enjoyed the interactions with many users of this representation of photon cross sections, starting with the original publication in February of 1967 and continuing through a revision in December 1971. As always, we sincerely appreciate user feedback. 


\section{Contents}

1. Introduction ....................................................................................................................................................... 9

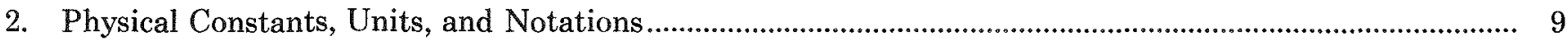

2.1 Fundamental Constants ..................................................................................................................... 9

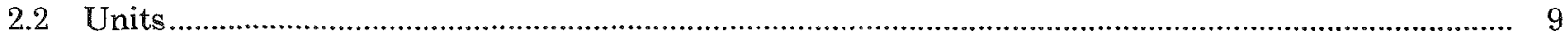

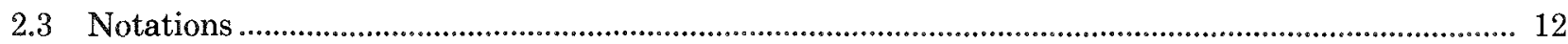

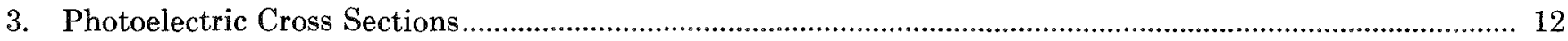

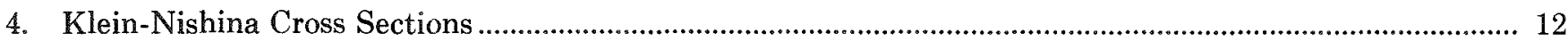

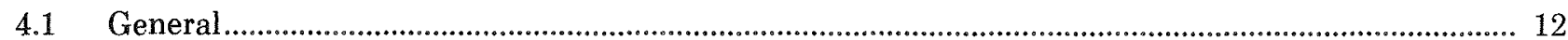

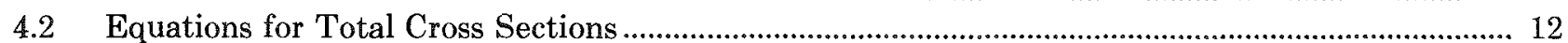

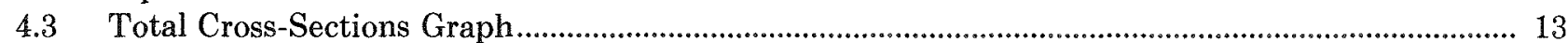

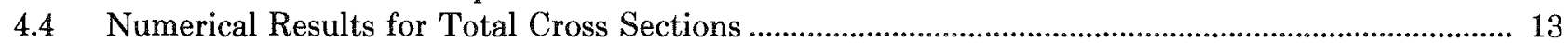

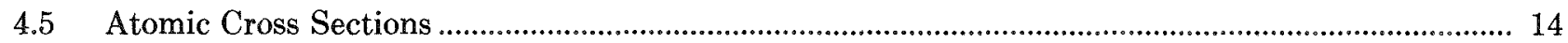

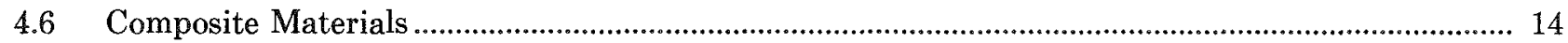

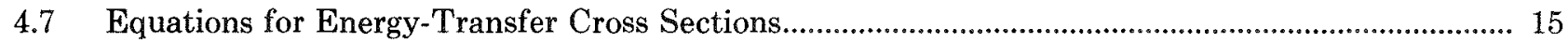

4.8 Graphs of Energy-Transfer Approximations............................................................................. 15

4.9 Numerical Results for Energy-Transfer Approximations ............................................................. 16

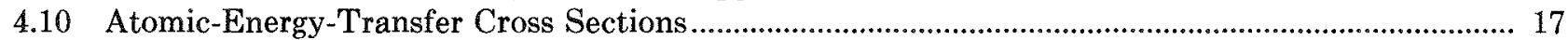

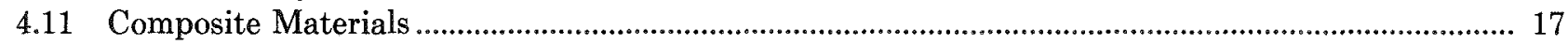

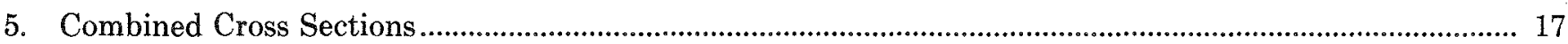

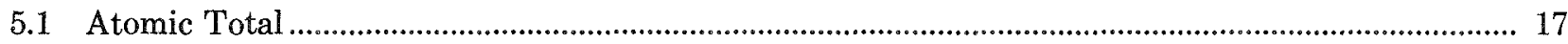

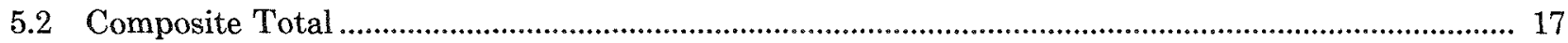

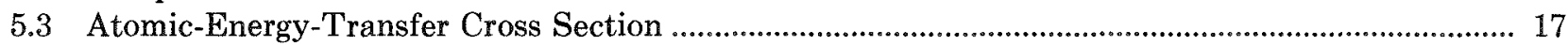

5.4 Composite-Energy-Transfer Cross Section.................................................................................... 17

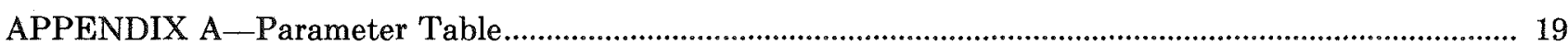

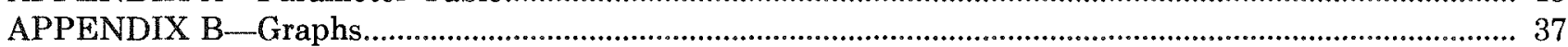

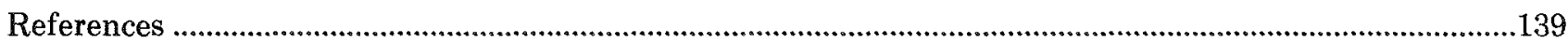




\section{Analytical Approximations for X-Ray Cross Sections III}

\section{Introduction}

This report updates our earlier analytical representation of cross sections for both photoelectric absorption of photons by atoms and Klein-Nishina scattering of photons from atoms. ${ }^{1}$ We updated the photoelectric cross sections to include new data that have become available in the last 15 years. The largest changes occur at photon energies below $1 \mathrm{keV}$.

There is now greater interest in the user community for convenient computer approximations for cross sections at higher photon energies than existed before. Therefore, we have added analytical representations for the Klein-Nishina cross sections that apply at higher photon energies than we included in our earlier report.

We tried to keep this document reasonably selfcontained. This means that some material is reproduced here that also appears in our earlier publications. ${ }^{1-2}$

This document is more likely to be used as a reference that is referred to periodically than read only one time from cover to cover. We constructed the Contents with this in mind so it can easily be used to locate topics of interest. The graphs are expected to get the most use; they are at the end of the document, with the element name and corresponding atomic number across the top of the page for easy reference. As in previous revisions, the values of the absorption edges are printed on the graphs.

This new compilation has been used here at Sandia for over a year to help check it for errors. Therefore, any new results that became available during this time were not included in the fitting.

The report is organized as follows. Section 2 gives the physical constants used in the report, defines units, provides some definitions, and gives a few notations. In Section 3 we discuss the form of the analytical representation for the photoelectric cross sections. Section 4 discusses the Klein-Nishina relations and our approximation to them. In Section 5 the combined cross sections show the result of adding the incoherent cross sections to the photoelectric cross sections, and we describe the application to composite materials. The parameter table in Appendix A gives the fitting parameters used in the analytical approximations for the photoelectric cross sections. Finally, the cross-section plots are provided in Appendix B. References are provided after the appendixes.

\section{Physical Constants, Units, and Notations}

\subsection{Fundamental Constants}

We provide the values of the physical constants to be used in this document here for convenient reference.

$$
\begin{aligned}
\mathrm{N}_{0} & =6.02205 \times 10^{23} / \mathrm{mole} \text { (Avogadro's number) } \\
\mathrm{r} & =2.81794 \times 10^{-13} \mathrm{~cm} \text { (electron radius) } \\
\mathrm{m} & =9.10953 \times 10^{-28} \mathrm{~g} \text { (electron mass) } \\
\mathrm{c} & =2.99792 \times 10^{10} \mathrm{~cm} / \mathrm{s} \text { (speed of light) }
\end{aligned}
$$

The numerical values of these constants are taken from Reference 3. Not all of these constants are used here to as many significant places as they are provided in the reference, but they are adequate for their use here.

A quantity that is used in the Klein-Nishina equations is

$\mathrm{L}=\frac{8}{3} \pi \mathrm{r}^{2} \mathrm{~N}_{0}=0.40061 \mathrm{~cm}^{2} /$ mole

\subsection{Units}

We will express atomic and composite-material cross sections in units of centimeters squared per gram. This is consistent with our earlier versions of this representation and is convenient for compatibility with existing computer codes that use this representation.

Although our final cross sections for any element or composite material are in units of centimeters squared per gram, it is convenient to first express the Klein-Nishina cross sections in units of centimeters squared per mole of electrons. Then, to get the Klein-Nishina approximation to the scattering cross section for an element of atomic number $\mathrm{Z}$ and atomic 
weight $\mathrm{A}$, we simply multiply by the ratio $\mathrm{Z} / \mathrm{A}$. This provides the corresponding atomic cross section in units of centimeters squared per gram.

In the Klein-Nishina equations and related results, the following substitution is used:

$\mathrm{X}=\frac{\mathrm{E}(\mathrm{keV})}{511.004}$ (photon energy in $\mathrm{mc}^{2}$ units).

Further substitutions used in developing the Klein-Nishina results include

$\omega=\frac{1}{(1+2 \mathrm{X})}$

and

$\eta=\frac{1}{2 \mathrm{X}}$
There are some constants appearing in this document that depend on the atomic weights of the elements. We tabulate these in Table 1 . Note that this table is divided into two sides, with 50 elements in each part. The atomic number $Z$ appears in the first column, the element symbol is given next in the El column, and the atomic weights are in the A column. The quantities in the Conv column are the multiplication factors needed for changing the units of photon cross sections from centimeters squared per gram to barns/atom $\left[10^{24} /\left(\mathrm{N}_{0} / \mathrm{A}\right)=\mathrm{A} / 0.602205\right]$. These conversions are also given for convenience below the plots of the photoelectric cross sections. Finally, the ratios of atomic number $(\mathrm{Z})$ to the atomic weight (A) are listed in the $\mathrm{Z} / \mathrm{A}$ columns. These numbers also appear in the fitting parameters table. The atomic weights given in Table 1 are from page B-3 of Reference 3 . There have been improvements of a few percent in the accuracy of some of these atomic weights since our last revision was published in 1971 . 
Table 1. Atomic Weights, Unit Conversions, and Z/A Ratios for the Elements

\begin{tabular}{|c|c|c|c|c|c|c|c|c|c|}
\hline $\mathrm{Z}$ & $\mathrm{EI}$ & A & Conv & $\mathrm{Z} / \mathrm{A}$ & $\mathrm{Z}$ & $\mathrm{El}$ & A & Conv & $\mathrm{Z} / \mathrm{A}$ \\
\hline 1 & $\mathrm{H}$ & 1.00794 & 1.67 & 0.9921 & 51 & $\mathrm{Sb}$ & 121.75 & 202.17 & 0.4189 \\
\hline 2 & $\mathrm{He}$ & 4.00260 & 6.65 & 0.4997 & 52 & $\mathrm{Te}$ & 127.60 & 211.89 & 0.4075 \\
\hline 3 & $\mathrm{Li}$ & 6.941 & 11.53 & 0.4322 & 53 & I & 126.9045 & 210.73 & 0.4176 \\
\hline 4 & $\mathrm{Be}$ & 9.01218 & 14.97 & 0.4438 & 54 & $\mathrm{Xe}$ & 131.29 & 218.02 & 0.4113 \\
\hline 5 & B & 10.81 & 17.95 & 0.4625 & 55 & $\mathrm{Cs}$ & 132.9054 & 220.70 & 0.4138 \\
\hline 6 & $\mathrm{C}$ & 12.011 & 19.95 & 0.4995 & 56 & $\mathrm{Ba}$ & 137.33 & 228.05 & 0.4078 \\
\hline 7 & $\mathrm{~N}$ & 14.0067 & 23.26 & 0.4998 & 57 & La & 138.9055 & 230.66 & 0.4104 \\
\hline 8 & 0 & 15.9994 & 26.57 & 0.5000 & 58 & $\mathrm{Ce}$ & 140.12 & 232.68 & 0.4139 \\
\hline 9 & $\mathbf{F}$ & 18.99840 & 31.55 & 0.4737 & 59 & $\operatorname{Pr}$ & 140.9077 & 233.99 & 0.4187 \\
\hline 10 & $\mathrm{Ne}$ & 20.179 & 33.51 & 0.4956 & 60 & $\mathrm{Nd}$ & 144.24 & 239.52 & 0.4160 \\
\hline 11 & $\mathrm{Na}$ & 22.98977 & 38.18 & 0.4785 & 61 & $\mathrm{Pm}$ & 145. & 240.78 & 0.4207 \\
\hline 12 & $\mathrm{Mg}$ & 24.305 & 40.36 & 0.4937 & 62 & $\mathrm{Sm}$ & 150.36 & 249.68 & 0.4123 \\
\hline 13 & $\mathrm{Al}$ & 26.98154 & 44.80 & 0.4818 & 63 & $\mathrm{Eu}$ & 151.96 & 252.34 & 0.4146 \\
\hline 14 & $\mathrm{Si}$ & 28.0855 & 46.64 & 0.4985 & 64 & $\mathrm{Gd}$ & 157.25 & 261.12 & 0.4070 \\
\hline 15 & $\mathrm{P}$ & 30.97376 & 51.43 & 0.4843 & 65 & $\mathrm{~Tb}$ & 158.9254 & 263.91 & 0.4090 \\
\hline 16 & $\mathrm{~S}$ & 32.06 & 53.24 & 0.4991 & 66 & Dy & 162.50 & 269.84 & 0.4062 \\
\hline 17 & $\mathrm{Cl}$ & 35.453 & 58.87 & 0.4795 & 67 & Ho & 164.9304 & 273.88 & 0.4062 \\
\hline 18 & $\mathrm{Ar}$ & 39.948 & 66.34 & 0.4506 & 68 & $\mathrm{Er}$ & 167.26 & 277.75 & 0.4066 \\
\hline 19 & $\mathrm{~K}$ & 39.0983 & 64.93 & 0.4860 & 69 & $\mathrm{Tm}$ & 168.9342 & 280.53 & 0.4084 \\
\hline 20 & $\mathrm{Ca}$ & 40.08 & 66.56 & 0.4990 & 70 & $\mathrm{Yb}$ & 173.04 & 287.34 & 0.4045 \\
\hline 21 & $\mathrm{Sc}$ & 44.9559 & 74.65 & 0.4671 & 71 & $\mathrm{Lu}$ & 174.967 & 290.54 & 0.4058 \\
\hline 22 & $\mathrm{Ti}$ & 47.88 & 79.51 & 0.4595 & 72 & Hf & 178.49 & 296.39 & 0.4034 \\
\hline 23 & V & 50.9415 & 84.59 & 0.4515 & 73 & $\mathrm{Ta}$ & 180.9479 & 300.48 & 0.4034 \\
\hline 24 & $\mathrm{Cr}$ & 51.996 & 86.34 & 0.4616 & 74 & W & 183.85 & 305.29 & 0.4025 \\
\hline 25 & $\mathrm{Mn}$ & 54.9380 & 91.23 & 0.4551 & 75 & $\operatorname{Re}$ & 186.207 & 309.21 & 0.4028 \\
\hline 26 & $\mathrm{Fe}$ & 55.847 & 92.74 & 0.4656 & 76 & Os & 190.2 & 315.84 & 0.3996 \\
\hline 27 & $\mathrm{Co}$ & 58.9332 & 97.86 & 0.4581 & 77 & Ir & 192.22 & 319.19 & 0.4006 \\
\hline 28 & $\mathrm{Ni}$ & 58.69 & 97.46 & 0.4771 & 78 & $\mathrm{Pt}$ & 195.08 & 323.94 & 0.3998 \\
\hline 29 & $\mathrm{Cu}$ & 63.546 & 105.52 & 0.4564 & 79 & $\mathrm{Au}$ & 196.9665 & 327.08 & 0.4011 \\
\hline 30 & $\mathrm{Zn}$ & 65.38 & 108.57 & 0.4589 & 80 & $\mathrm{Hg}$ & 200.59 & 333.09 & 0.3988 \\
\hline 31 & $\mathrm{Ga}$ & 69.72 & 115.77 & 0.4446 & 81 & $\mathrm{Tl}$ & 204.383 & 339.39 & 0.3963 \\
\hline 32 & $\mathrm{Ge}$ & 72.59 & 120.54 & 0.4408 & 82 & $\mathrm{~Pb}$ & 207.2 & 344.07 & 0.3958 \\
\hline 33 & As & 74.9216 & 124.41 & 0.4405 & 83 & $\mathrm{Bi}$ & 208.9804 & 347.03 & 0.3972 \\
\hline 34 & $\mathrm{Se}$ & 78.96 & 131.12 & 0.4306 & 84 & Po & 209. & 347.06 & 0.4019 \\
\hline 35 & $\mathrm{Br}$ & 79.904 & 132.69 & 0.4380 & 85 & At & 210. & 348.72 & 0.4048 \\
\hline 36 & $\mathrm{Kr}$ & 83.80 & 139.16 & 0.4296 & 86 & $\mathrm{Rn}$ & 222. & 368.65 & 0.3874 \\
\hline 37 & $\mathrm{Rb}$ & 85.4678 & 141.92 & 0.4329 & 87 & $\mathrm{Fr}$ & 223. & 370.31 & 0.3901 \\
\hline 38 & $\mathrm{Sr}$ & 87.62 & 145.50 & 0.4337 & 88 & $\mathrm{Ra}$ & 226.0254 & 375.33 & 0.3893 \\
\hline 39 & $\mathrm{Y}$ & 88.9059 & 147.63 & 0.4387 & 89 & Ac & 227.0278 & 376.99 & 0.3920 \\
\hline 40 & $\mathrm{Zr}$ & 91.22 & 151.48 & 0.4385 & 90 & $\mathrm{Th}$ & 232.0381 & 385.31 & 0.3879 \\
\hline 41 & $\mathrm{Nb}$ & 92.9064 & 154.28 & 0.4413 & 91 & $\mathrm{~Pa}$ & 231.0359 & 383.65 & 0.3939 \\
\hline 42 & Mo & 95.94 & 159.31 & 0.4378 & 92 & $\mathrm{U}$ & 238.0289 & 395.26 & 0.3865 \\
\hline 43 & $\mathrm{Tc}$ & 98. & 162.74 & 0.4388 & 93 & $\mathrm{~Np}$ & 237.0482 & 393.6 & 0.3923 \\
\hline 44 & $\mathrm{Ru}$ & 101.07 & 167.83 & 0.4353 & 94 & $\mathrm{Pu}$ & 244. & 405.18 & 0.3852 \\
\hline 45 & $\mathrm{Rh}$ & 102.9055 & 170.88 & 0.4373 & 95 & $\mathrm{Am}$ & 243. & 403.52 & 0.3909 \\
\hline 46 & $\mathrm{Pd}$ & 106.42 & 176.72 & 0.4322 & 96 & $\mathrm{Cm}$ & 247. & 410.16 & 0.3887 \\
\hline 47 & $\mathrm{Ag}$ & 107.8682 & 179.12 & 0.4357 & 97 & $\mathrm{Bk}$ & 247. & 410.16 & 0.3927 \\
\hline 48 & $\mathrm{Cd}$ & 112.41 & 186.66 & 0.4270 & 98 & $\mathrm{Cf}$ & 251. & 416.80 & 0.3904 \\
\hline 49 & In & 114.82 & 190.67 & 0.4268 & 99 & $\mathrm{Es}$ & 252 & 418.46 & 0.3929 \\
\hline 50 & $\mathrm{Sn}$ & 118.69 & 197.09 & 0.4213 & 100 & $\mathrm{Fm}$ & 257. & 426.77 & 0.3891 \\
\hline
\end{tabular}




\subsection{Notations}

We use the subscript $i$ to denote atomic number and $f_{i}$ to denote the mass fraction of element $i$ in $a$ composite material. The index $\mathrm{j}$ is used to denote the $j$-th energy interval for fitting the photoelectric cross sections. We use a subscript $\mathrm{c}$ to denote a composite material.

\section{Photoelectric Cross Sections}

The representation for the photoelectric cross section in interval $j$ of element $i$ is the linear combination of reciprocal powers of the photon energy $E(E$ in $\mathrm{keV}$ ).

$\mu_{i j}=\frac{A_{i j 1}}{E}+\frac{A_{i j 2}}{E^{2}}+\frac{A_{i j 3}}{E^{3}}+\frac{A_{i j 4}}{E^{4}}$,

$i=1, \ldots, 100 ; j=1, \ldots, m_{i}$

where $m_{i}$ is the number of fitting intervals used for element $i$. The units are centimeters squared per gram for the photoelectric cross section $\mu$. The fitting parameters $\mathrm{A}_{\mathrm{ijk}}$ are tabulated in Appendix A.3.

The fitting of these parameters to source data is discussed in Appendix B, where the cross-section plots are presented.

\section{Klein-Nishina Cross Sections}

\subsection{General}

The Klein-Nishina relations are used in this representation to estimate incoherent scattering. The equations used here are based on the theory developed in Evans. ${ }^{4}$

The Klein-Nishina model applies to free stationary electrons. Of course, atomic electrons are neither free nor stationary. However, the corrections for these effects are small compared to the sum of the photoelectric plus incoherent cross sections. For example, at small photon energies where electron binding effects are important, photoelectric effects dominate the total photon cross section. On the other hand, at high energies where the photoeffect is a small part of the total, the atomic binding effects become negligible compared to the energy of the incident photon.
In this section we first treat the total KleinNishina cross sections; then we discuss the KleinNishina energy-transfer cross sections. In each case the equations are given first, followed by graphs of the equations and a table of numerical results.

When discussing Klein-Nishina cross sections as applied to electrons, we use units of centimeters squared per mole $\left(6.02205 \times 10^{23}\right)$ of electrons. Constants and variable changes $\mathrm{X}, \omega$, and $\eta$ are defined in Section 2.2 by Eqs (2), (3), and (4).

\subsection{Equations for Total Cross Sections}

The theoretical result for the total Klein-Nishina cross section is

$$
\begin{aligned}
R= & \frac{3}{4} L\left[\left(\frac{2+2 X-X^{2}}{2 X^{3}}\right) \ln \omega\right. \\
& \left.+2 \omega \frac{(1+X)^{2}}{X^{2}}-\omega^{2}(1+3 X)\right] .
\end{aligned}
$$

The power-series expansion of this is sometimes a useful approximation at small photon energies and shows the form of the low-energy limit of the equation

$\mathrm{R} \approx \mathrm{L}\left(1-2 \mathrm{X}+\frac{26}{5} \mathrm{X}^{2}-\frac{133}{10} \mathrm{X}^{3}+\frac{1144}{35} \mathrm{X}^{4}-\ldots\right)$

It is convenient to have a simple approximation to $\mathrm{R}$ that can be evaluated adequately in single precision on a 32-bit computer and that will use less machine time than the full equation for $\mathrm{R}$. One such approximation for $\mathrm{R}$ is the following rational function that was used in our earlier compilations.

$\mathrm{R} \approx \mathrm{L}\left(\frac{1+1.148 \mathrm{X}+0.06141 \mathrm{X}^{2}}{1+3.171 \mathrm{X}+0.9328 \mathrm{X}^{2}+0.02572 \mathrm{X}^{3}}\right)$

This is a good approximation to the KleinNishina equation for photon energies below $10 \mathrm{MeV}$.

An approximation for $R$ valid at large values of $X$ is

$$
\begin{aligned}
\mathrm{R} \approx & \frac{3}{8} \mathrm{~L} \eta\left[2\left(1-4 \eta-8 \eta^{2}\right) \ln (1+2 \mathrm{X})\right. \\
& \left.+1+16 \eta-\eta^{2}\right] .
\end{aligned}
$$


We recommend using this approximation to evaluate scattering cross sections for photons with energies above $10 \mathrm{MeV}$.

\subsection{Total Cross-Sections Graph}

Figure 1 shows a graph of the total Klein-Nishina cross section in units of centimeters squared per mole of electrons versus photon energy in kiloelectronvolts. The solid curve shows $\mathrm{R}$ of $\mathrm{Eq}(6)$, the rational fraction of $\mathrm{Eq}(8)$ by the short-dashed curve, the highenergy approximation of Eq (9) by the long-dashed curve, and the power series expansion of $\mathrm{Eq}(7)$ by the dash-dot-dot-dot curve as indicated in the legend of the figure. Note that the rational function approximation falls below the true value above about 10 $\mathrm{MeV}$. However, the high-energy approximation is very good in this interval.

Of course, one could just use the complete KleinNishina equation at all energies, but it requires multiple precision word-length evaluation at low values of photon energies and is slower to evaluate than are the approximations. We recommend using the rational function ( $\mathrm{Eq}(8)$ ) for photon energies up to $10 \mathrm{MeV}$ and the high-energy approximation ( $\mathrm{Eq}(9))$ for photon energies above $10 \mathrm{MeV}$.

\subsection{Numerical Results for Total Cross Sections}

The purpose of Table 2 is to give values of the total Klein-Nishina cross sections more accurately than can be read from a graph and to extend the evaluations to higher photon energies. Also, the relative errors of the corresponding approximations are provided. The values of the Klein-Nishina cross sections in units of centimeters squared per mole of electrons are given versus photon energy in kiloelectronvolts in the $\mathrm{R}$ column of Table 2 as determined by the multiple-precision evaluation of $\mathrm{Eq}(6)$. The Rea column gives the relative (fractional) error of the rational function approximation of $R$ by $\mathrm{Eq}$ (8), and the Rel column gives the relative errors for the large energy approximation of $\mathrm{R}$ by $\mathrm{Eq}$ (9). The relative errors in the series expansion using the number of terms shown in $\mathrm{Eq}(7)$ are given in the last column.

Note that when a relative error exceeds $100 \%$, we simply designate this by the entry "large," and when it is $<10^{-6}$ we indicate it by the entry "small." The exact values of the errors in these approximations are not of interest whenever they are extremely small or large enough to render the approximation useless.

As stated previously, we recommend using Eq (8) to approximate the total Klein-Nishina cross sections for photon energies below $10 \mathrm{MeV}$ and using $\mathrm{Eq}$ (9) for photon energies above $10 \mathrm{MeV}$.

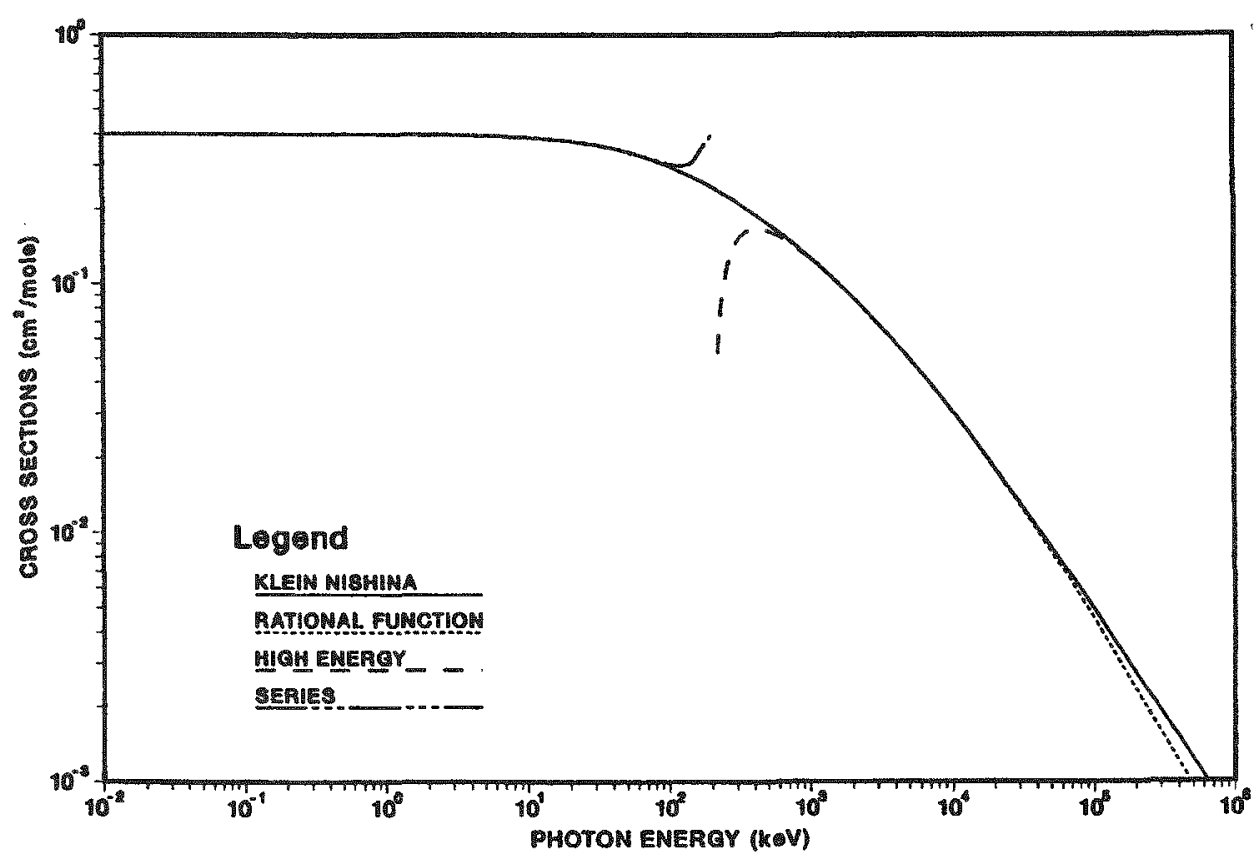

Figure 1. Klein-Nishina Total Cross Sections 


\section{Table 2. Values $\mathrm{R}$ of Eq (6) and Relative Errors Rea of Eq (8), Rel of Eq (9), and Res of Eq (7) vs Photon Energy E}

\begin{tabular}{|c|c|c|c|c|}
\hline $\mathrm{E}(\mathrm{keV})$ & $\mathrm{R}$ & Rea & Rel & Res \\
\hline $1 \times 10^{-2}$ & $4.006 \times 10^{-1}$ & small & large & small \\
\hline $4 \times 10^{-2}$ & $4.005 \times 10^{-1}$ & small & large & small \\
\hline $6 \times 10^{-2}$ & $4.005 \times 10^{-1}$ & small & large & small \\
\hline $1 \times 10^{-1}$ & $4.004 \times 10^{-1}$ & $-4.5 \times 10^{-6}$ & large & small \\
\hline $4 \times 10^{-1}$ & $4.000 \times 10^{-1}$ & $-1.8 \times 10^{-5}$ & large & small \\
\hline $6 \times 10^{-1}$ & $3.997 \times 10^{-1}$ & $-2.7 \times 10^{-5}$ & large & small \\
\hline $1 \times 10^{0}$ & $3.990 \times 10^{-1}$ & $-4.4 \times 10^{-5}$ & large & small \\
\hline $4 \times 10^{0}$ & $3.945 \times 10^{-1}$ & $-1.6 \times 10^{-4}$ & large & small \\
\hline $6 \times 10^{0}$ & $3.915 \times 10^{-1}$ & $-2.3 \times 10^{-4}$ & large & small \\
\hline $1 \times 10^{1}$ & $3.857 \times 10^{-1}$ & $-3.5 \times 10^{-4}$ & large & $2.1^{-6}$ \\
\hline $4 \times 10^{1}$ & $3.485 \times 10^{-1}$ & $-5.9 \times 10^{-4}$ & large & $2.1^{-4}$ \\
\hline $6 \times 10^{1}$ & $3.286 \times 10^{-1}$ & $-4.4 \times 10^{-4}$ & large & $1.7^{-3}$ \\
\hline $1 \times 10^{2}$ & $2.967 \times 10^{-1}$ & $5.2 \times 10^{-5}$ & large & $2.1^{-2}$ \\
\hline $1 \times 10^{2}$ & $1.907 \times 10^{-1}$ & $3.4 \times 10^{-4}$ & large & large \\
\hline $6 \times 10^{2}$ & $1.611 \times 10^{-1}$ & $-2 \times 10^{-4}$ & $-3.4 \times 10^{-1}$ & large \\
\hline $1 \times 10^{3}$ & $1.272 \times 10^{-1}$ & $-3.6 \times 10^{-4}$ & $-5.8 \times 10^{-2}$ & large \\
\hline $4 \times 10^{3}$ & $6.931 \times 10^{-2}$ & $-1.3 \times 10^{-4}$ & $-5.1 \times 10^{-4}$ & large \\
\hline $6 \times 10^{3}$ & $4.410 \times 10^{-2}$ & $-1.1 \times 10^{-4}$ & $-1.3 \times 10^{-4}$ & large \\
\hline $1 \times 10^{4}$ & $2.071 \times 10^{-2}$ & $-7.5 \times 10^{-4}$ & small & large \\
\hline $4 \times 10^{4}$ & $1.052 \times 10^{-2}$ & $-2.8 \times 10^{-2}$ & small & large \\
\hline $6 \times 10^{4}$ & $7.553 \times 10^{-3}$ & $-5 \times 10^{-2}$ & small & large \\
\hline $1 \times 10^{5}$ & $4.937 \times 10^{-3}$ & $-8.7 \times 10^{-2}$ & small & large \\
\hline $4 \times 10^{5}$ & $1.505 \times 10^{-3}$ & $-2.1 \times 10^{-1}$ & small & large \\
\hline $6 \times 10^{5}$ & $1.056 \times 10^{-3}$ & $-2.4 \times 10^{-1}$ & small & large \\
\hline $1 \times 10^{6}$ & $6.729 \times 10^{-4}$ & $-2.8 \times 10^{-1}$ & small & large \\
\hline $4 \times 10^{6}$ & $1.949 \times 10^{-4}$ & $-3.7 \times 10^{-1}$ & small & large \\
\hline $6 \times 10^{6}$ & $1.351 \times 10^{-5}$ & $-4 \times 10^{-1}$ & small & large \\
\hline $1 \times 10^{7}$ & $8.501 \times 10^{-5}$ & $-4.3 \times 10^{-1}$ & small & large \\
\hline $4 \times 10^{7}$ & $2.391 \times 10^{-5}$ & $-4.9 \times 10^{-1}$ & small & large \\
\hline $6 \times 10^{7}$ & $1.646 \times 10^{-5}$ & $-5.1 \times 10^{-1}$ & small & large \\
\hline $1 \times 10^{8}$ & $1.027 \times 10^{-5}$ & $-5.2 \times 10^{-1}$ & small & large \\
\hline
\end{tabular}

\subsection{Atomic Cross Sections}

The incoherent scattering cross section, in units of centimeters squared per gram for an element of atomic number i (using the Klein-Nishina approximation) is

$\eta_{\mathrm{i}}=\left(\frac{\mathrm{Z}}{\mathrm{A}}\right)_{\mathrm{i}} \mathrm{R}$

where $\mathrm{Z}$ is the atomic number and $\mathrm{A}$ is the atomic weight of element $i$. The $Z$ /A ratios are tabulated in Table 1, and R should be used from $\mathrm{Eq}(8)$ or $\mathrm{Eq}(9)$.

\subsection{Composite Materials}

The total incoherent scattering cross section in units of centimeters squared per gram for a composite material consisting of $\mathrm{N}$ elements is

$\eta_{\mathrm{c}}=\sum_{\mathrm{i}=1}^{\mathrm{N}} \mathrm{f}_{\mathrm{i}} \eta_{\mathrm{i}}=\left(\frac{\mathrm{Z}}{\mathrm{A}}\right)_{\mathrm{c}} \mathrm{R}$ 
where

$\left(\frac{\mathrm{Z}}{\mathrm{A}}\right)_{\mathrm{c}}=\sum_{\mathrm{i}=1}^{\mathrm{N}} \mathrm{f}_{\mathrm{i}}\left(\frac{\mathrm{Z}}{\mathrm{A}}\right)_{\mathrm{i}}$.

The parameter $f_{i}$ is the fraction (by mass) of element $i$ in the composite material.

\subsection{Equations for Energy- Transfer Cross Sections}

The Klein-Nishina cross section for transferring energy to the scattering electrons is

$$
\begin{aligned}
S= & \frac{3}{4} L\left\{\frac{3+2 X-X^{2}}{2 X^{3}} \ln \omega+\frac{2 \omega(1+X)^{2}}{X^{2}}\right. \\
& -\omega^{2}\left[(1+3 X)+\frac{(1+X)\left(2 X^{2}-2 X-1\right)}{X^{2}}\right] \\
& \left.-\frac{4}{3} X^{2} \omega^{3}\right\},
\end{aligned}
$$

where $\mathrm{X}$ is the photon in energy in $\mathrm{mc}^{2}$ units (see Eq (2)), the constant L is defined by Eq (1), and the change in variable $\omega$ is defined by $\mathrm{Eq}$ (3).

The power-series expansion for $\mathbf{S}$ is

$\mathrm{S} \approx \mathrm{L}\left(\mathrm{X}-\frac{42}{10} \mathrm{X}^{2}+\frac{147}{10} \mathrm{X}^{3}-\frac{1616}{35} \mathrm{X}^{4}+\ldots\right)$
A useful rational function approximation for photon $<10 \mathrm{MeV}$ is the one used in our earlier work.

$\mathrm{S} \approx \mathrm{L}\left(\frac{\mathrm{X}+0.825 \mathrm{X}^{2}+0.03234 \mathrm{X}^{3}}{1+5.393 \mathrm{X}+5.212 \mathrm{X}^{2}+0.8783 \mathrm{X}^{3}+0.01599 \mathrm{X}^{4}}\right)$.

However, at photon energies higher than $10 \mathrm{MeV}$ we recommend the use of the following approximation:

$$
\begin{aligned}
\mathrm{S} \approx & \frac{3}{8} \mathrm{~L} \eta\left[2\left(1-4 \eta-12 \eta^{2}\right) \ln (1+2 \mathrm{X})\right. \\
& \left.-\frac{5}{3}+22 \eta+\eta^{2}\right]
\end{aligned}
$$

where $\eta$ is defined in Section 2.2 above by Eq (4).

\subsection{Graphs of Energy-Transfer Approximations}

Figure 2 shows the Klein-Nishina energy-transfer cross section Eq (13) in units of centimeters squared per mole of electrons versus photon energy in kiloelectronvolts by the solid curve. The approximations discussed above are also shown as indicated in the legend of the figure. Note that the rationalfunction approximation $\mathrm{Eq}$ (15) falls below the correct value above about $\mathbf{E}=10^{4} \mathrm{keV}$. However, the high-energy approximation ( $\mathrm{Eq}(16))$ is good in this interval.

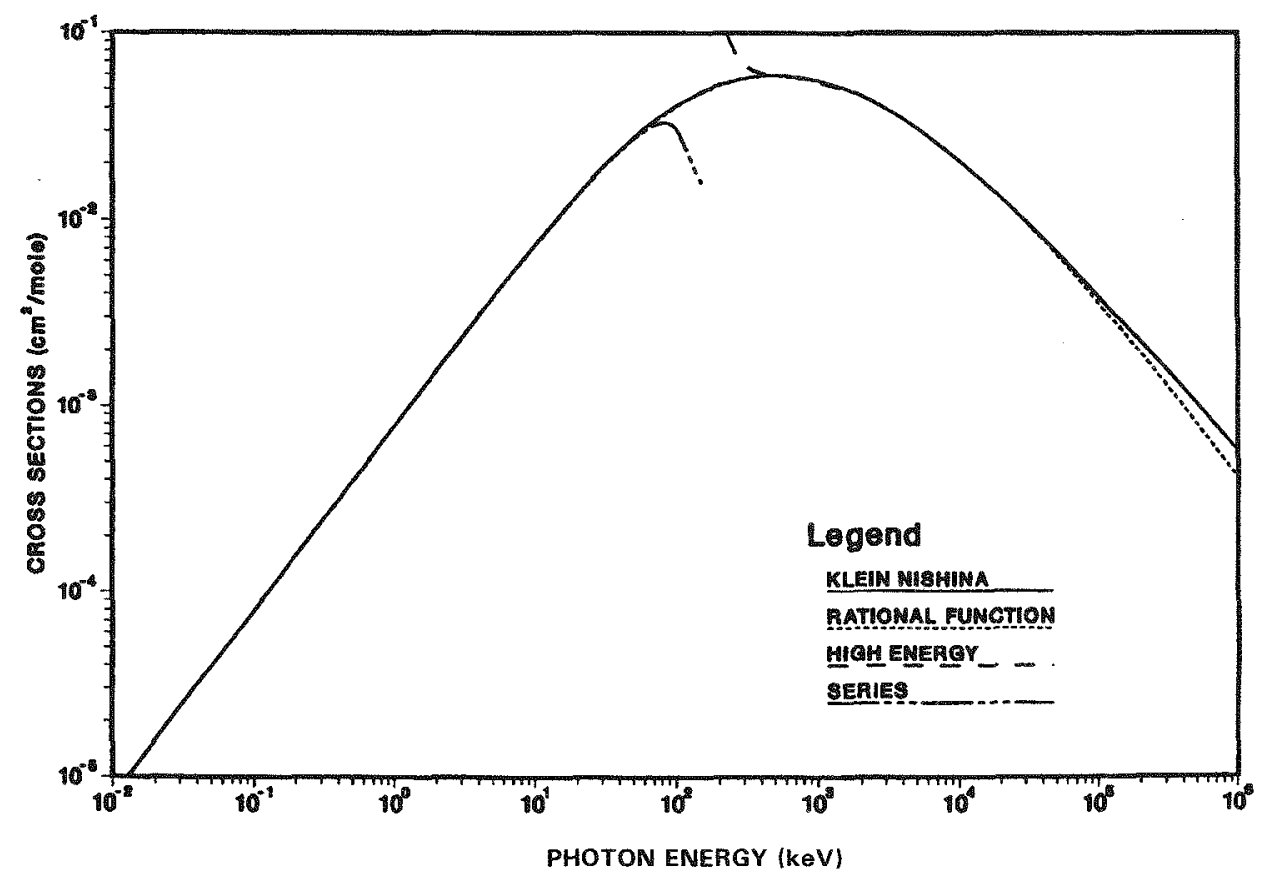

Figure 2. Klein-Nishina Energy-Transfer Cross Sections 


\subsection{Numerical Results for Energy-Transfer Approximations}

The purpose of Table 3 is to provide some values of the Klein-Nishina cross sections more accurately than can be read from a graph, to tabulate the relative errors in the corresponding approximations, and to extend the evaluations to higher photon energies. The values of the Klein-Nishina energy-transfer cross sections in units of centimeters squared per mole of electrons appear in column $\mathrm{S}$ versus the photon energy kiloelectronvolts given in the first column as obtained by multiple precision evaluation of $\mathrm{Eq}$ (13).
The relative (fractional) errors in the rational-fraction approximation ( $\mathrm{Eq}(15))$ are given in the Rea column, the relative errors in the high-energy approximation (Eq (16)) are given in the Rel column, and the relative errors in the power-series expansion of $\mathrm{Eq}(14)$ appear in the last column.

When a relative error in Table 3 exceeds $100 \%$, we designate it by the entry "large" and when it is $<10^{-6}$ by the entry "small." Again, we recommend using $\mathrm{Eq}$ (15) as an approximation for the KleinNishina energy-transfer cross sections for photon energies below $10 \mathrm{MeV}$ and using $\mathrm{Eq}$ (16) for photon energies $\geq 10 \mathrm{MeV}$.

\section{Table 3. Values S of Eq (12), Relative Errors Rea of Eq (14), Rel of Eq (15), and Res of Eq (13) vs Photon Energy E}

\begin{tabular}{|c|c|c|c|c|}
\hline $\mathrm{E}(\mathrm{keV})$ & $\mathrm{S}$ & Rea & Rel & Res \\
\hline $1 \times 10^{-2}$ & $7.839 \times 10^{-6}$ & small & large & small \\
\hline $4 \times 10^{-2}$ & $3.135 \times 10^{-5}$ & small & large & small \\
\hline $6 \times 10^{-2}$ & $4.701 \times 10^{-5}$ & small & large & small \\
\hline $1 \times 10^{-1}$ & $7.833 \times 10^{-5}$ & $-7.2 \times 10^{-5}$ & large & small \\
\hline $4 \times 10^{-1}$ & $3.126 \times 10^{-4}$ & $-2.8 \times 10^{-4}$ & large & small \\
\hline $6 \times 10^{-1}$ & $4.681 \times 10^{-4}$ & $-4.3 \times 10^{-4}$ & large & small \\
\hline $1 \times 10^{\circ}$ & $7.775 \times 10^{-4}$ & $-7.1 \times 10^{-4}$ & large & small \\
\hline $4 \times 10^{\circ}$ & $3.035 \times 10^{-3}$ & $-2.7 \times 10^{-3}$ & large & small \\
\hline $6 \times 10^{0}$ & $4.481 \times 10^{-3}$ & $-3.9 \times 10^{-3}$ & large & small \\
\hline $1 \times 10^{1}$ & $7.237 \times 10^{-3}$ & $-6.1 \times 10^{-3}$ & large & $-2.0 \times 10^{-5}$ \\
\hline $4 \times 10^{1}$ & $2.331 \times 10^{-2}$ & $-1.5 \times 10^{-2}$ & large & $-5.6 \times 10^{-3}$ \\
\hline $6 \times 10^{1}$ & $3.076 \times 10^{-2}$ & $-1.7 \times 10^{-2}$ & large & $-2.9 \times 10^{-2}$ \\
\hline $1 \times 10^{2}$ & $4.095 \times 10^{-2}$ & $-1.6 \times 10^{-2}$ & large & $-2.4 \times 10^{-1}$ \\
\hline $4 \times 10^{2}$ & $5.905 \times 10^{-2}$ & $3.8 \times 10^{-4}$ & $3.7 \times 10^{-2}$ & large \\
\hline $6 \times 10^{2}$ & $5.920 \times 10^{-2}$ & $1.6 \times 10^{-3}$ & $-1.7 \times 10^{-3}$ & large \\
\hline $1 \times 10^{3}$ & $5.597 \times 10^{-2}$ & $6.8 \times 10^{-4}$ & $-2.0 \times 10^{-3}$ & large \\
\hline $4 \times 10^{3}$ & $3.508 \times 10^{-2}$ & $-1.4 \times 10^{-5}$ & $-3.5 \times 10^{-5}$ & large \\
\hline $6 \times 10^{3}$ & $2.840 \times 10^{-2}$ & $-2.4 \times 10^{-5}$ & $-9.3 \times 10^{-6}$ & large \\
\hline $1 \times 10^{4}$ & $2.099 \times 10^{-2}$ & $-8.1 \times 10^{-6}$ & small & large \\
\hline $4 \times 10^{4}$ & $7.993 \times 10^{-3}$ & $-1.8 \times 10^{-2}$ & small & large \\
\hline $6 \times 10^{4}$ & $5.863 \times 10^{-3}$ & $-3.8 \times 10^{-2}$ & small & large \\
\hline $1 \times 10^{5}$ & $3.919 \times 10^{-3}$ & $-7.3 \times 10^{-2}$ & small & large \\
\hline $4 \times 10^{5}$ & $1.250 \times 10^{-3}$ & $-2.0 \times 10^{-1}$ & small & large \\
\hline $6 \times 10^{5}$ & $8.854 \times 10^{-4}$ & $-2.4 \times 10^{-1}$ & small & large \\
\hline $1 \times 10^{6}$ & $5.706 \times 10^{-4}$ & $-2.9 \times 10^{-1}$ & small & large \\
\hline $4 \times 10^{6}$ & $1.693 \times 10^{-4}$ & $-3.9 \times 10^{-1}$ & small & large \\
\hline $6 \times 10^{6}$ & $1.181 \times 10^{-4}$ & $-4.2 \times 10^{-1}$ & small & large \\
\hline $1 \times 10^{7}$ & $7.476 \times 10^{-5}$ & $-4.5 \times 10^{-1}$ & small & large \\
\hline $4 \times 10^{7}$ & $2.136 \times 10^{-5}$ & $-5.2 \times 10^{-1}$ & small & large \\
\hline $6 \times 10^{7}$ & $1.476 \times 10^{-5}$ & $-5.3 \times 10^{-1}$ & small & large \\
\hline $1 \times 10^{8}$ & $9.246 \times 10^{-6}$ & $-5.5 \times 10^{-1}$ & small & large \\
\hline
\end{tabular}




\subsection{Atomic-Energy-Transfer Cross Sections}

The cross section in units of centimeters squared per gram for transfer of energy to an element of atomic number $i$ of the scattering medium is

$\sigma_{\mathrm{i}}=\left(\frac{\mathrm{Z}}{\mathrm{A}}\right)_{\mathrm{i}} \mathrm{S}$

where the ratio $\mathrm{Z} / \mathrm{A}$ is given in Table 1 and $\mathrm{S}$ is approximated by Eq (15) or Eq (16).

\subsection{Composite Materials}

For an $\mathrm{N}$-element composite material

$\sigma_{\mathrm{c}}=\sum_{\mathrm{i}=1}^{\mathrm{N}} \mathrm{f}_{\mathrm{i}} \sigma_{\mathrm{i}}=\left(\frac{\mathrm{Z}}{\mathrm{A}}\right)_{\mathrm{c}} \mathrm{S}$

where again $f_{i}$ is the mass fraction of element $i$ in the composite.

\section{Combined Cross Sections}

\subsection{Atomic Total}

The total cross section consists of the sum of the scattering cross section and the photoelectric cross section. Using the Klein-Nishina approximation for the scattering cross section gives

$\tilde{\mu}_{\mathrm{ij}}=\mu_{\mathrm{ij}}+\eta_{\mathrm{i}}$

for the total cross section in interval $j$ of element $i$.

\subsection{Composite Total}

For a composite material of $\mathrm{N}$ elements, the constituent atomic cross sections are averaged over the elements of the composite to get the cross section for the composite material.

$\left(\widetilde{\mu}_{\mathrm{j}}\right)_{\mathrm{c}}=\sum_{\mathrm{i}=1}^{\mathrm{N}} \mathrm{f}_{\mathrm{i}} \mu_{\mathrm{ij}}+\sum_{\mathrm{i}=1}^{\mathrm{N}} \mathrm{f}_{\mathrm{i}} \eta_{\mathrm{i}}$

The weighting factor $f_{i}$ in this average is the fraction (by mass) of element $i$ occurring in the composite material.

\subsection{Atomic-Energy-Transfer Cross Section}

The energy-transfer cross section for interval $j$ of element $i$ is

$\hat{\mu}_{\mathrm{ij}}=\mu_{\mathrm{ij}}+\sigma_{\mathrm{i}}$.

\subsection{Composite-Energy-Transfer Cross Section}

Again, for a composite material the atomic cross sections are averaged over the elements of the composite,

$\left(\hat{\mu}_{\mathrm{j}}\right)_{\mathrm{c}}=\sum_{\mathrm{i}=1}^{\mathrm{N}} \mathrm{f}_{\mathrm{i}} \mu_{\mathrm{ij}}+\sum_{\mathrm{i}=1}^{\mathrm{N}} \mathrm{f}_{\mathrm{i}} \sigma_{\mathrm{i}}$,

where again $f_{i}$ is the fraction (by mass) of element $i$ occurring in the composite material. 


\section{APPENDIX A}

\section{Parameter Table}

\section{A. 1 Disk Fille}

As discussed in Section 3, there are four fitting parameters in each of several intervals for each element as written in $\mathrm{Eq}(5)$. These fitting parameters are stored on a disk file named FRANK5.DAT. In this Appendix we describe the file containing the photoelectric fitting parameters. The format for reading this file is as follows: The first record contains only the integer -1 , and the second record contains only the integer 4 . These numbers are used by some of our existing codes. The third and fourth records identify the cross sections with the report number and date.

Record 5 begins the parameter table. There are several records (one for each fitting interval $j$ ) for each element for atomic number i between 1 and 100 . The atomic number is an integer and appears in the first three fields. The number of the fitting interval for a given element is an integer and appears in fields 4 through 6; this is the index $\mathrm{j}$ of Eq (5). Fields 7 through 14 contain the lower boundary of the fitting interval $\mathrm{j}$, fields 15 through 22 contain the upper boundary of fitting interval $j$, and fields 23 through 32 contain the atomic number to atomic weight ratio $\mathrm{Z} / \mathrm{A}$. Fields 33 through 45 contain the first fitting coefficient, fields 45 through 56 contain the second coefficient, fields 57 through 67 contain the third coefficient, and fields 68 through 78 contain the fourth and last of the photoelectric fitting parameters for element $\mathrm{i}$ and interval $\mathrm{j}$. The format for these records is: [FORMAT(2I3,2F8.0,E10.3,2X,4E11.3)].

\section{A. 2 Description of Table}

The representation scheme for computer evaluation of photoelectric cross sections involves the use of four fitting parameters in each of several intervals for each of 100 elements. These are the parameters contained in Eq (5) of Section 3. Note that the subscripts of the fitting parameters of $\mathrm{Eq}$ (5) are written in parentheses in the column headings of the parameter table. The first index I refers to the atomic number, which is written at the beginning of each subsection of the table just before the element name. The interval number $j$ appears in the first column of the table. The interval identification column is designated by INT IDENT and gives the interval boundaries in kiloelectronvolts. Some of the interval boundaries correspond to absorption edges. The RMS column gives the relative (fractional) root-meansquare error obtained when fitting $\mathrm{Eq}$ (5) to the source data. The entry NA is used in this column when the fitting was done without enough source data to make an adequate estimate of the fitting error. This occurs in some of the low-photon-energy intervals where data are scarce. The last column indicates whether or not a refitting was done in the corresponding intervals for this revision (update) by using $\mathrm{Y}$ (yes) and $N($ no) entries. Note that the ratio of atomic number to atomic weight $\mathrm{Z} / \mathrm{A}$ is also shown for each element. This multiplier is needed to multiply the Klein-Nishina results of Section 4 to obtain the scattering cross section for the corresponding atom in units of centimeters squared per gram. Since these constants are also given in Table 1 of Section II, it is less important than it was in the earlier versions of this compilation to provide them here in the Parameter Table. However, we felt that we should keep the format the same for the convenience of users already familiar with earlier versions.

\section{A.3 Table}

The parameter table is given on the next 16 pages. 
9 FLUORINE

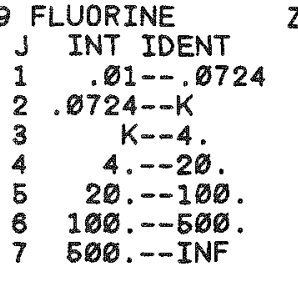

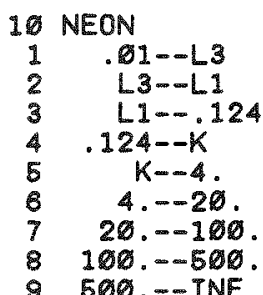

$\begin{array}{ccc}Z / A & =4.958 E-01 & \\ .01 & .0183 & N A \\ .0183 & .045 & N A \\ .045 & .124 & 3.2-2 \\ .124 & .887 & 1.8-2 \\ .887 & 4 . & 1.1-2 \\ 4 . & 2 \varnothing . & 4.5-3 \\ 20 . & 160 . & 4.6-3 \\ 100 . & 500 . & 3.3-2 \\ 500 . & \text { INF } & 7.7-2\end{array}$

$Z / A=4.737 E-61$

START FINISH

.8874 .

4.26.

20.100.

100.500

500. INF $\begin{array}{ll}.01 & .0724 \\ .0724 \quad .687\end{array}$

$Z / A=4.785 E-01$

81

.031 .63

.0631 .073

1.57320

20.106.

100. 500 .

ธซซ. INF

$Z / A=4.937 E-01$

$.01 \quad .051$

$.051 \quad .123$

$.123 \quad 1.365$

1.30520.

20.100.

100. 500 .

50ळ. INF

$20 .--100$

$500 .--$ INF

$Z / A=4.818 E-61$

.01 .0159

.0159 .073

$.073 \quad .1177$

$.1177 \quad 1.560$

1.58020

20. 100 .

100. 506.

500. INF

$100 .--500$.

500. - - INF

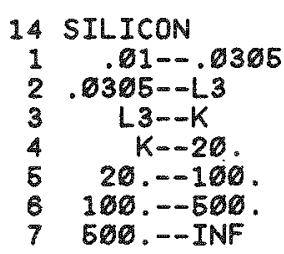

$Z / A=4.985 E-01$

.01 .0365

.0305 .099

$.099 \quad 1.839$

1.83920 .

20.100.

100.500

500. INF

$\begin{array}{rc}15 & \text { PHOSPHORUS } \\ 1 & .81--.03 \\ 2 & .0306--L 3 \\ 3 & L 3--K \\ 4 & K=-20 . \\ 5 & 20 .--100 \\ 6 & 100 .--500 \\ 7 & 500 .--I N F\end{array}$

$\begin{array}{ccc}Z / A & =4.843 E-01 & \\ .01 & .0308 & N A \\ .0306 & .132 & 5.1-3 \\ .132 & 2.144 & 6.5-2 \\ 2.144 & 20 . & 7.6-3 \\ 20 . & 100 . & 1.1-2 \\ 100 . & 500 . & 3.0-2 \\ 500 . & \text { INF } & 4.4-2\end{array}$

$Z / A=4.991 E-01$

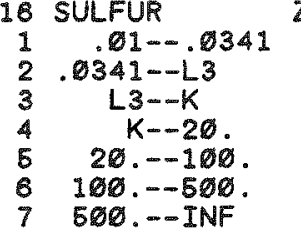

$.01, .0341$

$.185 \quad 2.472$

2.47220 .

20.100

100. 500

560 . INF

NA
NA
$5.5-2$
$1.4-2$
$8 \cdot 1-3$
$3.4-2$
$4.8-2$

$2.5-1$
$6.9-2$
$9.2-2$
$9.6-3$
$1.1-2$
$3.5-2$
$4.9-2$

1. $8-1$

1. 8-1

1. 2-1

1. $5-1$

1. -2

1.3-2

3.4-2

8. 2-2

NA

2. 2-6

1. $4-1$

$5.6-3$

1.1-2

3. 1-2

$4.7-2$

1. $0-2$

6. 5-2

$8.1-3$

1. $0-2$

4. 2-2

4.8-2

\begin{tabular}{lrl} 
RMS & \multicolumn{1}{c}{$A(I, J, 1)$} & \multicolumn{1}{l}{$A(I, J, 2)$} \\
NA & $1.129 E+\emptyset 4$ & $\emptyset$. \\
$1.2-2$ & $-2.878 E+\emptyset 2$ & $4.787 E+\emptyset 2$ \\
$1.8-2$ & $-8.907 E+\emptyset 1$ & $8.850 E+\varnothing 2$ \\
$8.3-3$ & $2.813 E+\emptyset 0$ & $-2.02 \varnothing E+\varnothing 2$ \\
$5.4-3$ & $6.097 E-\emptyset 2$ & $-2.435 E+\emptyset 1$ \\
$4.4-2$ & $4.857 E-\emptyset 3$ & $-1.8 \varnothing 5 E+\varnothing \emptyset$ \\
$9.7-2$ & $2.041 E-\emptyset 3$ & $2.257 E+\emptyset \emptyset$
\end{tabular}

$A(I, J, 3)$

b.

$8.787 E+101-4.504 E+100$

$7.217 E+03-2.022 E+03$

$9.903 E+03-4.454 E+03$

$6.099 E+03 \quad 2.258 E+04$

$3.335 E+03 \quad 1.285 E+05$

$1.684 E+163 \quad 5.997 E+25$

\begin{tabular}{|c|c|}
\hline $\begin{array}{r}1.006 \mathrm{E}-06 \\
8.235 \mathrm{E}+03 \\
-8.059 \mathrm{E}+03 \\
-1.940 \mathrm{E}+02 \\
-8.686 \mathrm{E}+01 \\
3.167 \mathrm{E}+00 \\
1.288 \mathrm{E}-01 \\
5.202 \mathrm{E}-103 \\
3.087 \mathrm{E}-03\end{array}$ & 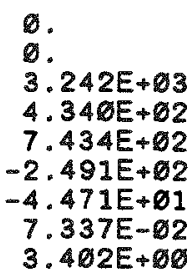 \\
\hline
\end{tabular}

0.

0.

$141 \mathrm{E}+90$

$2.357 E+02-1.666 E+\emptyset 1$

$1.077 E+04-3.822 E+03$

$1.439 E+04-8.187 E+03$

$9.862 E+\emptyset 3 \quad 2.497 E+\square 4$

$4.614 E+\emptyset 3 \quad 2.256 E+05$

$1.589 E+\emptyset 3 \quad 1.147 E+\emptyset 6$

5. $429 E+01$

$8.874 E+03$

$-5.565 E+182$

0.

$2.728 E-01-8.218 E+01$

$2.083 E-03 \quad 4.548 E+\varnothing 0$

4. $199 \mathrm{E}-13$

4. $808 E+90$

6.

$1.062 E+02-6.845 E+60$

$1.712 E+184-8.415 E+03$

$1.475 E+04 \quad 9.467 E+03$

$5.521 E+03 \quad 3.358 E+05$

$1.793 \mathrm{E}+63 \quad 1.898 \mathrm{E}+66$

$-5.574 E+\emptyset 2 \quad 8.887 E+\varnothing 1-1.493 E+\varnothing 0 \quad 8.669 E-\varnothing 3$ $-2.726 E+04 \quad 9.700 E+03-6.921 E+\varnothing 2 \quad 1.44 E E+\varnothing 1$ $-6.179 E+02 \quad 1.379 E+\varnothing 3 \quad 2.321 E+02-2.291 E+01$

$-6.648 E-01-1.084 E+02 \quad 2.219 E+\emptyset 4-1.241 E+\varnothing 4$

$4.080 E-01-1.205 E+02 \quad 2.139 E+04-1.143 E+02$

$8.338 E-03 \quad 2.236 E+\varnothing 0 \quad 8.819 E+\varnothing 3 \quad 4.284 E+\varnothing 5$

$6.233 E-\varnothing 3 \quad 7.344 E+\square 0$

$\begin{array}{ll}8.819 E+\varnothing 3 & 4.284 E+\emptyset 5 \\ 2.662 E+\$ 3 & 2.486 E+\$ 66\end{array}$

$-1.854 E+04 \quad 1.585 E+\varnothing 2$

$1.122 E+63-4.015 E+D 1$

$2.390 E+04-6.953 E+02$
$-5.284 E+02-1.399 E+03$

$\begin{array}{ll}-5.284 E+102 & 1.399 E+03 \\ -3.674 E+\square 0-1.622 E+\emptyset 1 & -1.30\end{array}$

4.158E- $01-1.351 E+\varnothing 2$

$1.125 E-D 2 \quad 2.747 E+D \emptyset$

$8.505 E-03 \quad 1.049 E+01$

3. $907 E+00-3.383 E-02$

$6.623 E-\emptyset 1-2.813 E-\emptyset 3$

$-7.978 E+\varnothing 1 \quad 1.974 E+\emptyset \emptyset$

$4.386 E+02-4.747 E+01$

$2.732 E+84-1.752 E+04$

$2.716 E+84 \quad 3.723 E+D 2$

$1.174 E+104 \quad 5.695 E+\varnothing 5$

$4.429 E+\varnothing 3 \quad 2.431 E+\varnothing 6$

$4.575 E+02$

$-1.168 E+02$

$-8.451 E+02$

$1.269 E+02-5$

0.

a.

$4.946 E-01-1.682 E+\emptyset 2$

$2.716 E-02-5.428 E+\varnothing 0$

$1.127 E-82 \quad 1.376 E+81$

$5.506 \mathrm{E}+00 \quad 6.616 \mathrm{E}-22$

$5.350 E+62-6.436 E+01$

$3.544 \mathrm{E}+04-2.595 \mathrm{E}+04$

$3.827 E+04-1.865 E+104$

$1.775 E+04 \quad 6.765 E+\varnothing 5$

$4.871 \mathrm{E}+03 \quad 4.477 \mathrm{E}+16$

4.728E+ +2

b.

$-5.700 \mathrm{E}+02 \quad 1.909 \mathrm{E}+03$

$-6.098 E+00 \quad 7.712 E+01$

5.710E- $01-2.001 E+02$

$3.613 E-02-8.426 E+D 0$

$1.421 E-\varnothing 2 \quad 1.77 \varnothing E+\varnothing 1$

$-1.122 E+1$.

$1.589 E-61$

$4.196 E+04-3.437 E+04$

$4.505 E+04-3.853 E+04$

$2.277 E+\emptyset 4 \quad 8.080 E+25$

$6.221 E+13 \quad 5.554 E+\varnothing 6$
$5.368 E+102 \varnothing$.

$-4.88$

$-1.021 E+01$

$7.467 E-01-2.534 E+D 2$

$6.338 E-02-3.443 E+01$

$1.857 \mathrm{E}-\emptyset 2 \quad 2.398 \mathrm{E}+\emptyset 1$
0
$1.815 E+01 \quad 2.796 E-01$

$1.206 E+03-1.934 E+02$

$5.164 E+104-4.707 E+64$

$5.806 E+84-6.891 E+04$

$4.658 E+04 \quad 2.233 E+185$

$6.766 E+03 \quad 8.358 E+26$ 


$\begin{array}{rcclc}17 & \text { CHLORINE } & \text { Z/A }=4.795 E-\emptyset 1 & \\ J & \text { INT IDENT } & \text { START } & \text { FINISH } & \text { RMS } \\ 1 & .01--.0436 & .01 & .0436 & \text { NA } \\ 2 & .0438--L 3 & .0436 & .200 & 1.8-1 \\ 3 & \text { L3--K } & .200 & 2.824 & 3.1-2 \\ 4 & \text { K--20. } & 2.824 & 20 . & 7.5-3 \\ 5 & 20 .--100 . & 20 . & 100 . & 1.1-2 \\ 6 & 100 .--500 . & 100 . & 560 . & 3.1-2 \\ 7 & 500 .--I N F & 500 . & \text { INF } & 4.3-2\end{array}$

$\begin{array}{rccll}18 & A R G O N & Z / A & =4.506 E-\emptyset 1 & \\ 1 & .01--.03 \emptyset 2 & .01 & .0302 & N A \\ 2 & .0302-L 3 & .0302 & .245 & 4.1-2 \\ 3 & L 3--K & .245 & 3.203 & 1.3-1 \\ 4 & K--20 . & 3.293 & 20 . & 5.3-3 \\ 5 & 20 .--100 . & 20 . & 100 . & 8.5-3 \\ 6 & 100 .--500 . & 100 . & 500 . & 2.5-2 \\ 7 & 500 .--I N F & 500 . & \text { INF } & 3.9-2\end{array}$

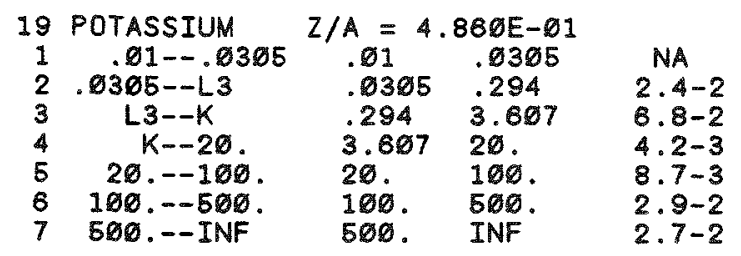

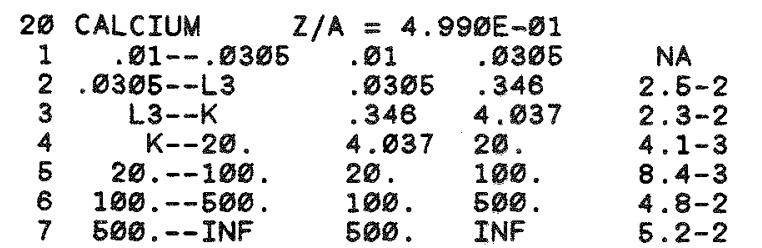

$\begin{array}{rccll}21 & \text { SCANDIUM } & \text { Z/A }=4.871 E-\emptyset 1 \\ 1 & .01--M 3 & .01 & .032 & 6.0-3 \\ 2 & M 3--L 3 & .032 & .402 & 4.6-2 \\ 3 & \text { L3--K } & .402 & 4.491 & 5.4-2 \\ 4 & K--1 \varnothing 0 . & 4.491 & 190 . & 8.3-3 \\ 5 & 100 .--500 . & 160 . & 500 . & 6.2-3 \\ 6 & 500 .--I N F & 500 . & \text { INF } & 3.0-2\end{array}$

$\begin{array}{rccl}22 & \text { TITANIUM } & Z / A=4.595 E-\emptyset 1 \\ 1 & .01--.1 & .01 & .1 \\ 2 & .1--L 3 & .1 & .456 \\ 3 & \text { L3--K } & .456 & 4.966 \\ 4 & K--100 . & 4.966 & 100 . \\ 5 & 100 .--500 . & 100 . & 500 . \\ 6 & 500 .--I N F & 500 . & \text { INF }\end{array}$

$\begin{array}{rccll}23 & \text { VANADIUM } & Z / A=4.515 E-\emptyset 1 \\ 1 & .01--.12 & .01 & .12 & 1.3-1 \\ 2 & .12--L 3 & .12 & .513 & 1.1-2 \\ 3 & \text { L3--L1 } & .513 & .628 & 4.1-2 \\ 4 & \text { LI--K } & .828 & 5.485 & 2.4-2 \\ 5 & \text { K--100. } & 5.465 & 100 . & 8.2-3 \\ 6 & 100 .--500 . & 100 . & 500 . & 7.2-3 \\ 7 & 500 .--I N F & 500 . & \text { INF } & 6.6-2\end{array}$

\begin{tabular}{|c|c|c|c|c|}
\hline $\begin{array}{r}24 \\
1 \\
2 \\
3 \\
4 \\
5 \\
8\end{array}$ & $\begin{array}{c}\text { CHROMIUM } \\
.01--.106 \\
.106--L 3 \\
L 3--K \\
K-100 . \\
100 .--500 . \\
500 .--I N F\end{array}$ & $\begin{array}{c}Z / A=4 . \\
.01 \\
.108 \\
.575 \\
5.989 \\
100 . \\
500 .\end{array}$ & $\begin{array}{l}18 E-01 \\
.106 \\
.575 \\
5.989 \\
100 . \\
500 . \\
\text { INF }\end{array}$ & $\begin{array}{l}1 \cdot 2-1 \\
1 \cdot 2-2 \\
4 \cdot 9-2 \\
8 \cdot 4-2 \\
9.9-3 \\
7.2-2\end{array}$ \\
\hline $\begin{array}{l}1 \\
2 \\
3 \\
4 \\
5\end{array}$ & $\begin{array}{c}\text { MANGANESE } \\
.01--.1 \\
.1--L 3 \\
L 3--K \\
K-100 . \\
100 .--500 . \\
500 .--I N F\end{array}$ & $\begin{array}{c}2 / A=4 \\
.01 \\
.1 \\
.640 \\
8.539 \\
100 . \\
500 .\end{array}$ & $\begin{array}{l}551 E-01 \\
.1 \\
.640 \\
6.539 \\
100 . \\
500 . \\
\text { INF }\end{array}$ & $\begin{array}{l}8 \cdot 1-2 \\
1 \cdot 0-2 \\
3 \cdot y-2 \\
7.1-3 \\
9.3-3 \\
6.2-2\end{array}$ \\
\hline
\end{tabular}

$8.6-2$

1. $1-2$

$2.4-2$

8. $3-3$

$1.2-2$

$4.6-2$

\begin{tabular}{|c|c|c|c|}
\hline $\begin{array}{r}A(I, J, I) \\
7.8 \emptyset 8 E+\varnothing 2 \\
-8.163 E+\emptyset 1 \\
-2.147 E+02 \\
-1.192 E+\emptyset 1 \\
8.789 E-01\end{array}$ & $\begin{array}{l}A(I, J, 2) \\
\emptyset . \\
3.934 E+\emptyset 2 \\
1.289 E+\emptyset 3 \\
3.253 E+\emptyset 2 \\
-2.920 E+\emptyset 2 \\
-2.175 E+D 1 \\
2.884 E+\emptyset 1\end{array}$ & $\begin{array}{l}A(I, J, 3) \\
0 . \\
-2.563 E+01 \\
2.099 E+\emptyset 3 \\
5.927 E+\emptyset 4 \\
6.880 E+04 \\
3.895 E+\emptyset 4 \\
9.717 E+\varnothing 3\end{array}$ & $\begin{array}{r}A(I, J, 4) \\
0 . \\
4.396 E-01 \\
-3.705 E+02 \\
-5.986 E+104 \\
-1.018 E+65 \\
9.737 E+65 \\
9.001 E+66\end{array}$ \\
\hline
\end{tabular}

$1.275 E+\emptyset 4 \varnothing$.

$-1.674 E+03 \quad 1.047 E+13-9.010 E+101 \quad 2.166 E+00$

$-2.244 E+02 \quad 1.388 E+03 \quad 2.571 E+03-5.113 E+82$

$-1.446 E+01 \quad 4.359 E+02 \quad 6.578 E+84-7.284 E+04$

$8.786 E-11-3.061 E+02 \quad 7.682 E+04-1.219 E+05$

$6.779 E-02-2.895 E+01 \quad 5.069 E+04 \quad 5.281 E+105$

$2.598 E-\emptyset 2 \quad 3.454 E+\emptyset 1$

$1.059 E+\varnothing 4 \quad 1.068 E+07$

$3.111 E+\emptyset 3 \emptyset$

$6.162 E+11 \quad 5.776 E+12-4.497 E+01 \quad 9.218 E-01$

$-3.601 E+02 \quad 2.287 E+03 \quad 2.513 E+03-4.591 E+02$

$-2.113 E+01 \quad 7.161 E+102 \quad 8.183 E+04-9.859 E+04$

$1.048 E+D 0-3.644 E+02 \quad 9.844 E+\emptyset 4-1.760 E+05$

$1.046 E-01-3.553 E+01 \quad 6.096 E+04 \quad 1.226 E+06$

$3.453 E-\emptyset 2 \quad 4.621 E+\varnothing 1$

$1.869 E+04 \quad 1.238 E+07$

$\begin{array}{rr}3.029 E+\emptyset 3 & \emptyset \\ 3.816 E+\emptyset 2 & 5.887 E+\emptyset 2 \\ -1.054 E+\emptyset 2 & 1.120 E+\emptyset 3 \\ -3.015 E+\emptyset 1 & 1.141 E+\emptyset 3 \\ 1.408 E+\emptyset 0 & -4.553 E+\emptyset 2 \\ 1.204 E-01 & -5.356 E+\emptyset 1 \\ 4.178 E-\emptyset 2 & 5.761 E+\emptyset 1\end{array}$

.

$888 E+011.019 E+\varnothing 0$

$5.115 E+03-1.325 E+03$

$9.459 E+04-1.195 E+05$

$1.206 E+85-2.531 E+05$

$8.779 E+04 \quad 6.252 E+05$

$1.377 E+04 \quad 2.019 E+07$

$1.121 E+83$

$2.976 E+02$

$-1.567 \mathrm{E} \rightarrow 02$

$4.833 E-02 \quad 6.881 E+1$

$-3.020 E-\emptyset 1 \quad 1.510 E-03$

$-4.935 E+\varnothing 1 \quad 1.044 E+\emptyset \emptyset$

$5.139 E+03-1.442 E+03$

$1.217 E+05-2.057 E+05$

$9.253 E+1.096 E+16$

$2.762 E+\varnothing 4 \quad 1.290 E+\emptyset 7$

$3.596 E+03-6.877 E+01$

$-3.132 E+02 \quad 1.624 E+03$

$9.609 E-01-4.754 E-83$

$-4.628 E+01 \quad 8.678 E+02$

$2.074 E-01-2.292 E+02$

$1.972 E-01-9.550 E+81$

(7.096E+

$7.308 E+03-2.383 E+03$

$1.371 E+05-2.489 E+05$

$1.106 \mathrm{E}+05 \quad 1.067 \mathrm{E}+06$

$5.606 E-02 \quad 8.140 E+\emptyset 1$

3.106E+ $1.587 E+\$ 7$

$4.117 E+03-1.054 E+02 \quad 1.531 E+80-7.725 E-03$ $-4.052 E+02 \quad 1.169 E+03-1.212 E+02 \quad 4.148 E+00$

$9.968 E+03-1.796 E+03-1.264 E+03 \quad 1.481 E+03$

$-1.429 E+02 \quad 1.632 E+03 \quad 7.203 E+93-2.283 E+03$

$3.940 E-01-2.733 E+02 \quad 1.564 E+05-3.184 E+05$

$2.007 E-91-8.911 E+01 \quad 1.254 E+95 \quad 1.137 E+66$

$6.449 E-02 \quad 9.483 E+61 \quad 3.684 E+04 \quad 1.821 E+07$

$5.069 E+03-7.968 E+01 \quad 8.395 E-01-4.494 E-53$ $-4.404 E+02 \quad 1.301 E+03-1.166 E+02 \quad 3.481 E+00$

$3.932 E+01 \quad 1.537 E+12 \quad 1.228 E+04-4.984 E+83$

$2.740 E-81-2.665 E+02 \quad 1.818 E+85-3.997 E+85$

$2.727 E-11-1.288 E+02 \quad 1.543 E+05 \quad 9.755 E+\emptyset 5$

$7.660 E-12 \quad 1.142 E+62$

$4.290 E+104$

$2.161 E+97$

$5.692 E+03-1.278 E+02 \quad 1.150 E+\varnothing 0-3.774 E-03$ $-4.901 E+\varnothing 2 \quad 1.450 E+\emptyset 3-1.365 E+02 \quad 4.134 E+\varnothing 0$ $-3.065 E+\varnothing 1 \quad 7.209 E+\varnothing 2 \quad 1.275 E+\varnothing 4-5.462 E+\emptyset 3$ $2.930 E-01-2.703 E+82 \quad 2.034 E+85-4.886 E+05$ $2.940 E-91-1.342 E+62 \quad 1.761 E+65 \quad 8.747 E+65$ $8.736 E-02 \quad 1.322 E+02 \quad 4.952 E+04 \quad 2.436 E+107$
$U$
$Y$
$Y$
$Y$
$N$
$N$
$Y$

$Y$
$Y$
$Y$
$N$
$N$
$N$
$Y$




$$
\begin{array}{cccc}
26 & \text { IRON } & \text { Z/A }=4.856 E-01 \\
J & \text { INT IDENT } & \text { START } & \text { FINISH } \\
1 & .01--.024 & .01 & .024 \\
2 & .024-.12 & .024 & .12 \\
3 & .12--L 3 & .12 & .768 \\
4 & \text { L3--K } & .708 & 7.112 \\
5 & K--100 & 7.112 & 106 . \\
6 & 100 .--500 . & 100 . & 500 . \\
7 & 500 .--I N F & 500 . & \text { INF }
\end{array}
$$$$
27 \text { COBALT } \quad Z / A=4.581 E-61
$$$$
\begin{array}{lc}
1 & .01--.0308 \\
2 & .0308-. .093 \\
3 & .093--L 3 \\
4 & L 3--K \\
5 & K=-100 . \\
6 & 100 .--500 . \\
7 & 500 .--I N F
\end{array}
$$

$\begin{array}{ll}.01 & .0308 \\ .6308 & .093 \\ .093 & .779 \\ .779 & 7.769 \\ 7.709 & 100 . \\ 100 . & 500 . \\ 506 . & \text { INF }\end{array}$

NA
$1.9-2$
$5.1-2$
$3.8-2$
$6.9-3$
$6.3-3$
$4.0-2$

28 NICKEL

$$
\begin{aligned}
& 28 \\
& 1 \\
& 2 \\
& 3 \\
& 4 \\
& 5 \\
& 6 \\
& 7 \\
& 8
\end{aligned}
$$

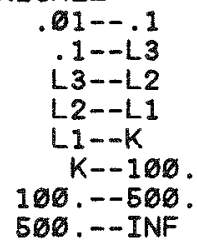

INF

\section{COPPER $Z / A=4.564 E-\varnothing 1$}

1
2
3
4
5
6

$$
\begin{gathered}
.01--.1 \\
.1--L 3 \\
L 3--L 1 \\
L 1--K \\
K--100 . \\
100 .--500 . \\
500 .--I N F
\end{gathered}
$$

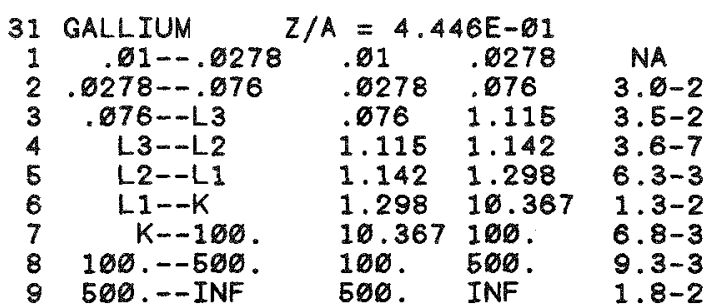

\begin{tabular}{rcclc}
32 & GERMANIUM & \multicolumn{4}{c}{$Z / A=4.408 E-01$} \\
1 & $.01-.0305$ & .01 & .0305 & NA \\
2 & $.0305-.1$ & .0305 & 1 & $7.9-3$ \\
3 & $.1--L 3$ & .1 & 1.217 & $3.4-2$ \\
4 & $L 3--L 2$ & 1.217 & 1.248 & $2.2-7$ \\
5 & $L 2--L 1$ & 1.248 & 1.413 & $2.1-6$ \\
6 & $L 1--K$ & 1.413 & 11.104 & $1.1-2$ \\
7 & $K--100$. & 11.104 & 100. & $5.7-3$ \\
8 & $100 .--500$. & 100. & 500. & $9.1-3$ \\
9 & $500 .--I N F$ & 500. & INF & $1.9-2$
\end{tabular}

6. 6-2

4. 4-2

$\emptyset$.

$9.6-3$

$5.4-3$

5. 5-3

$4.7-2$

1.5-1

$6.5-2$

7. $4-7$

1. $1-2$

5. $6-3$

$7.0-3$

6.5-2
NA

. 3-2

$4.4-7$

1.2-2

$7-3$
$.8-3$

8-2

$4-2$

. $2-7$

$1-2$

. 1-3

RMS
$3 \cdot 9-2$
$1 \cdot 6-1$
$5 \cdot 6-2$
$2 \cdot 0-2$
$5 \cdot 4-3$
$7.0-3$
$6 \cdot 4-2$

$\begin{array}{rr}A(I, J, 1) & A(I, J, 2) \\ -3.328 E+\emptyset 3 & 2.590 E+\emptyset 2 \\ 4.132 E+\emptyset 3 & 3.963 E+\emptyset 2 \\ -2.683 E+\emptyset 1 & 1.250 E+\emptyset 3 \\ -8.128 E+\emptyset 0 & 8.550 E+\emptyset 2 \\ 2.151 E-01 & -2.880 E+\varnothing 2 \\ 3.511 E-\emptyset 1 & -1.833 E+\emptyset 2 \\ 1.026 E-\emptyset 1 & 1.672 E+\emptyset 2\end{array}$

$A(I, J, 3)$

$-3.812 E+60$

$-2.505 E+101$

$-7.321 E+\square 1$

$1.511 E+04$

$2.341 E+05$

$2.098 E+\emptyset 5$
$5.981 E+04$

$A(I, J, 4)$

3. $437 E-01$

$1.137 E+60$

$-7.079 E+\square 3$

$-6.083 E+65$

$7.131 E+05$

$2.806 E+67$

$2.074 E+03$ ஜ

年

$-8.228 E+02 \quad 1.982 E+03-1.782 E+02 \quad 4.937 E+60$

$2.092 E+01 \quad 3.311 E+02 \quad 1.820 E+04-9.303 E+03$

$4.947 E-02-2.478 E+\emptyset 2 \quad 2.586 E+\varnothing 5-7.185 E+05$

$3.588 E-01-1.492 E+02 \quad 2.298 E+05 \quad 8.856 E+05$

$1.154 E-01 \quad 1.796 E+\emptyset 2$

$8.515 E+03-2.121 E+102$ $-1.021 E+03 \quad 2.388 E+03$

D.

๑.

$-1.175 E+22 \quad 2.171 E+03$

$1.685 E-02-2.522 E+02$

$4.681 E-01-2.585 E+62$

$1.364 \mathrm{E}-1012.148 \mathrm{E}+02$

$9.992 E+03-3.780 E+02$ $-8.896 E+102 \quad 2.391 E+03$ $-5.089 E+162 \quad 4.139 E+03$ $-9.173 E+01 \quad 1.862 E+93$

$7.440 E-63-2.371 E+102$

$4.729 E-01-2.055 E+02$

$1.472 E-81 \quad 2.345 E+22$

$1.479 E+63$.

$1.325 E+04-6.762 E+02$ $-1.336 E+03 \quad 3.104 E+03$ $-2.807 E+84$

$-3.057 E+03$

$-8.018 E+01$

$-1.158 E+00-3.871 E+01$

$4.820 E-\emptyset 1-1.832 E+02$

$1.669 E-01 \quad 2.696 E+02$

$1.479 E+103$

$1.211 E+04-7.144 E+102$

$-1.199 E+03 \quad 3.215 E+03$

$-1.457 E+04 \quad 6.363 E+04$

$3.470 E+\varnothing 3-8.674 E+\emptyset 3$

$-9.431 E+01 \quad 2.078 E+83$

$-1.253 E+00-2.897 E+\varnothing 1$

5.591E-01-2.217E+ 02

1.949E-01 2.371E+02

$4.941 E+63$

$1.269 E+04-9.339 E+02$ $-1.358 E+03 \quad 3.695 E+03$ $-7.402 E+183$

$-3.400 E+02$

$-5.940 E+91$ I. $340 E+83$

$-2.025 E+001.283 E+62$

$6.478 E-01-2.603 E+02$

$2.155 E-61 \quad 2.609 E+02$
3. $100 \mathrm{E}+07$

$6.740 E+184$

$2.132 E+60-7.667 E-03$ $-2.361 E+\emptyset 2 \quad 7.261 E+\emptyset 0$

0.

๑.

$1.514 E+04-6.042 E+03$

$3.005 E+05-9.000 E+05$

$2.782 E+\varnothing 5 \quad 4.399 E+05$

$8.639 E+104 \quad 3.626 E+07$

$5.657 E+100-2.788 E-62$ $-1.986 E+02 \quad 3.649 E+00$ $7.468 E+03-6.981 E+02$

$1.777 E+04-8.202 E+03$

$3.202 E+\varnothing 5-1.028 E+106$

$3.060 E+05 \quad 2.182 E+05$

$8.644 E+104 \quad 3.990 E+67$

๑.

$1.188 E+\emptyset 1-8.785 E-02$ $-3.692 E+62 \quad 8.946 E+00$ $-1.511 E+D 5 \quad 7.897 E+04$ $3.780 E+02-2.183 E+02$ $2.695 E+04-1.070 E+04$ $3.499 E+65-1.154 E+06$ $3.296 E+\emptyset 5 \quad 6.802 E+05$ $1.000 E+\varnothing 5 \quad 4.382 E+\emptyset 7$

0. $3.594 E+02 \quad 1.152 E+01$ $-1.011 E+65 \quad 6.416 E+04$ $2.548 E+104-8.411 E+03$ $2.172 E+104-1.101 E+04$ $3.783 E+185-1.357 E+06$ $3.625 E+05 \quad 3.392 E+05$

$1.767 \mathrm{E}+\$ 5 \quad 2.468 \mathrm{E}+07$

D.

$2.767 E+01-1.952 E-01$ $-4.569 E+02 \quad 1.599 E+01$ $-6.676 E+04 \quad 5.346 E+104$ $1.163 E+04-1.089 E+03$ $2.621 E+154-1.507 E+104$ 4. $069 E+05-1.510 E+06$ $4.045 E+05-5.153 E+04$ $2.074 E+65 \quad 2.226 E+67$

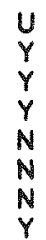

$Y$
$Y$
$Y$
$N$
$N$
$N$
$Y$

$Y$
$Y$
$N$
$N$
$N$
$N$
$N$
$Y$

$Y$
$Y$
$N$
$N$
$N$
$Y$
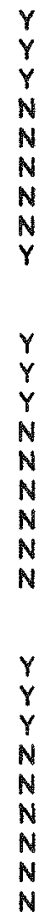
33 ARSENIC

\begin{tabular}{|c|c|c|c|}
\hline $\begin{array}{c}\text { ARSENIC } \\
\text { INT IDENT } \\
.01--.0371 \\
.0371--.1 \\
.1--L 3 \\
L 3--L 2 \\
\text { L2--L1 } \\
\text { L1--K } \\
K--100 . \\
100 .--500 . \\
500 .--I N F\end{array}$ & $\begin{array}{l}=4.40 \\
\text { START } \\
.01 \\
.0371 \\
.1 \\
1.323 \\
1.359 \\
1.529 \\
11.867 \\
160 . \\
500 .\end{array}$ & $\begin{array}{l}\text { FEE-O1 } \\
\text { FINISH } \\
.0371 \\
.1 \\
1.323 \\
1.359 \\
1.529 \\
11.867 \\
109 . \\
500 . \\
\text { INF }\end{array}$ & $\begin{array}{l}\text { RMS } \\
\text { NA } \\
4 \cdot 8-2 \\
3.7-2 \\
1.7-7 \\
1.4-6 \\
1.2-2 \\
6.4-3 \\
6.7-3 \\
1.8-2\end{array}$ \\
\hline
\end{tabular}

\section{4}

$Z / A=4.396 E-61$ .01 .0371

.371 .114

$.114 \quad 1.434$

$1.434 \quad 1.475$

$1.475 \quad 1.852$

$1.652 \quad 12.658$

$K--180 . \quad 12.858100$.

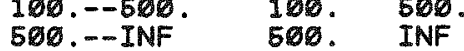

2. $4-2$

$3.8-2$

$2.7-8$

$1.4-6$

1. 1-2

7.9-3

1. $0 \mathrm{-2}$

1.9-2

35 BROMINE $Z / A=4.380 E-01$

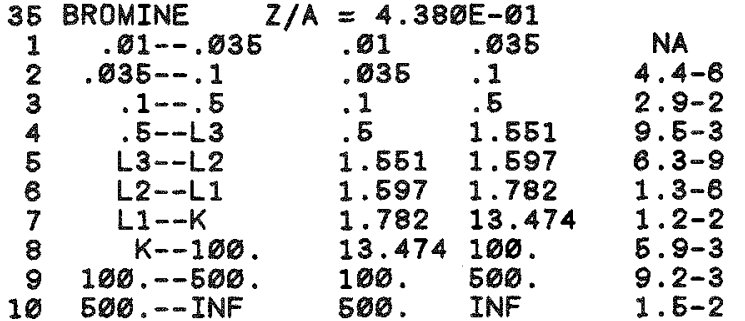

NA

4-6

. 5-3

2-2

9.2-3

1. $6-2$

36 KRYPTON $Z / A=4.296 E-\emptyset 1$

1
2
3
4
5
6
7
8
9
10

$\begin{array}{cll}.01--.0305 & .01 & .0305 \\ .0305-. .097 & .0365 & .097 \\ .097--.3 & .097 & .3 \\ .3--L 3 & .3 & 1.675 \\ L 3--L 2 & 1.875 & 1.727 \\ L 2--L 1 & 1.727 & 1.921 \\ \text { L1--K } & 1.921 & 14.323 \\ K--100 . & 14.323 & 100 . \\ 100 .-500 . & 109 . & 500 . \\ 560 .--I N F & 500 . & \text { INF }\end{array}$

NA

8.7-7

8.5-2

1. $3-1$

1. $8-8$

1. 2-6

$7.5-3$

$5.2-3$

6.8-3

1. $9-2$

\begin{tabular}{|c|c|c|c|c|}
\hline 3 & 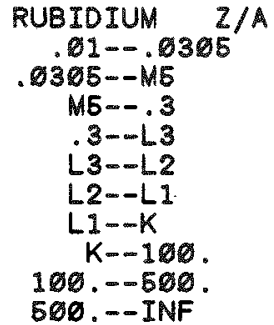 & $\begin{array}{l}=4.329 \\
.01 \\
.0305 \\
.111 \\
.3 \\
1.865 \\
1.883 \\
2.985 \\
15.200 \\
1006 \\
500 .\end{array}$ & $\begin{array}{l}\text { E- } 81 \\
.0305 \\
.111 \\
.3 \\
1.805 \\
1.863 \\
2.665 \\
15.206 \\
100 . \\
500 . \\
\text { INF }\end{array}$ & $\begin{array}{l}\text { NA } \\
1.4-1 \\
1.1-1 \\
1.2-1 \\
2.0-8 \\
1.0-8 \\
6.8-3 \\
6.3-3\end{array}$ \\
\hline
\end{tabular}

38 STRONTIUM $Z / A=4.337 E-11$

$\begin{array}{rcll}1 & .11--.0307 & .01 & .0307 \\ 2 & .0307--M 5 & .0307 & .133 \\ 3 & M 5--.3 & .133 & .3 \\ 4 & .3--L 3 & .3 & 1.94 \\ 5 & L 3--L 2 & 1.94 & 2.007 \\ 6 & L 2--L 1 & 2.097 & 2.216 \\ 7 & L 1--K & 2.216 & 16.105 \\ 8 & K--100 . & 18.105 & 100 . \\ 9 & 100 .-\infty 500 . & 100 . & 500 . \\ 10 & 560 .-- \text { INF } & 500 . & \text { INF }\end{array}$

NA

$1.8-1$

$7 \cdot 5-2$

1.2-1

$1.9-8$

$9.8-7$

6.9-3

$4 \cdot 6-3$

$2.6-3$

1. $8-2$

\begin{tabular}{|c|c|c|c|}
\hline $\begin{array}{l}A(I, J, I) \\
2.547 E+\emptyset 3 \\
2.492 E+\emptyset 4 \\
1.536 E+\emptyset 3 \\
4.525 E+\emptyset 3 \\
3.858 E+\emptyset 2 \\
5.339 E+\emptyset 1 \\
1.655 E+\emptyset 0 \\
7.886 E-\emptyset 1 \\
2.393 E-\emptyset 1\end{array}$ & $\begin{array}{r}A(I, J, 2) \\
\emptyset . \\
-3.110 E+\emptyset 3 \\
4.198 E+\emptyset 3 \\
2.468 E+04 \\
1 . \emptyset 13 E+\emptyset 4 \\
1.649 E+\emptyset 3 \\
8.588 E+\emptyset 1 \\
-3.490 E+\emptyset 2 \\
2.966 E+\varnothing 2\end{array}$ & $\begin{array}{l}A(I, J, 3) \\
\emptyset . \\
1.384 E+\emptyset 2 \\
-5.497 E+\emptyset 2 \\
-5.133 E+\emptyset 4 \\
3.603 E+03 \\
2.950 E+04 \\
4.504 E+\emptyset 5 \\
4.641 E+\emptyset 5 \\
2.380 E+\square 5\end{array}$ & $\begin{array}{r}A(I, J, 4) \\
0 . \\
-1.877 E+00 \\
1.920 E+61 \\
5.102 E+04 \\
-7.857 E+62 \\
-1.800 E+04 \\
-1.795 E+06\end{array}$ \\
\hline
\end{tabular}

$U$
$Y$
$Y$
$Y$
$N$
$N$
$N$
$N$
$N$
$N$

$1.677 \mathrm{E}+\varnothing 3 \curvearrowleft$.

$1.806 E+94-2.230 E+03 \quad 9.408 E+01-1.283 E+00$

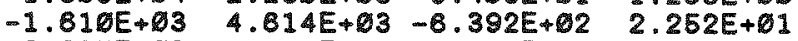

$-6.637 E+02 \quad 4.194 E+03-1.104 E+04 \quad 2.866 E+04$

$-4.265 E+02 \quad 9.860 E+63 \quad 5.343 E+63-1.174 E+03$

$-3.968 E+\emptyset 1 \quad 1.459 E+103 \quad 3.327 E+\emptyset 4-2.228 E+\varnothing 4$

$-3.461 E+00 \quad 4.401 E+\emptyset 2 \quad 4.690 E+05-1.943 E+\varnothing 6$

$8.564 E-01-3.723 E+02 \quad 5.020 E+05-1.430 E+08$

2. $590 E-\varnothing 1 \quad 3.237 E+\varnothing 2$

$\begin{array}{rr}5.820 E+05 & -1.430 E+06 \\ 2.541 E+05 & 2.321 E+07\end{array}$

$2.129 E+03 \square$.

$\varnothing$.

. $151 E+04-1.941 E+03 \quad 8.744 E+01-1.214 E+00$ $-3.534 E+13 \quad 6.788 E+03-1.134 E+03 \quad 5.165 E+01$

$-3.347 E+02 \quad 2.195 E+03 \quad 1.033 E+03-2.839 E+02$

$1.461 E+02-1.056 E+03 \quad 3.457 E+03 \quad 2.609 E+04$

$-4.318 E+02 \quad 1.067 E+04 \quad 6.548 E+03-1.535 E+03$

$-4.033 E+\emptyset 1 \quad 1.607 E+\emptyset 3 \quad 3.681 E+\emptyset 4-2.478 E+\emptyset 4$

$-3.955 E+00 \quad 5.482 E+02 \quad 5.202 E+05-2.262 E+06$

8.718E- $01-4.209 E+\varnothing 2$

$2.997 E-01 \quad 3.653 E+02$

$5.682 \mathrm{E}+05-2.099 \mathrm{E}+\varnothing 6$
$2.908 \mathrm{E}+\square 5$
$2.417 \mathrm{E}+07$

$3.691 E+\emptyset 3 \emptyset$.

6.

$2.024 E+03-2.083 E+02 \quad 4.439 E+00 \quad 1.056 E-01$

$\begin{array}{ll}7.848 E+02 & 5.257 E+03-9.960 E+02 \quad 4.686 E+01\end{array}$

$-2.451 E+02 \quad 2.199 E+03 \quad 1.217 E+83-3.120 E+02$

$3.611 E+02-2.974 E+03 \quad 1.308 E+04 \quad 1.268 E+04$

$-4.183 E+02 \quad 1.017 E+04 \quad 7.535 E+03-1.891 E+03$

$-9.924 E-01 \quad 8.455 E+02 \quad 4.446 E+04-3.490 E+04$

$-3.976 E+100 \quad 4.257 E+02 \quad 5.613 E+05-2.587 E+\varnothing 6$

$1.050 E+10-4.435 E+02 \quad 6.114 E+05-3.028 E+06$

$3.136 E-101 \quad 3.952 E+162$

$3.252 E+165$

$2.248 E+67$

$\begin{array}{lll}4.606 E+03 & 0 . & 0.021 E+00 \\ 1.441 E+03-1.761 E+02 & 6.821 E\end{array}$

$1.441 E+03-1.761 E+02 \quad 6.821 E+00 \quad 4.476 E-02$

$-6.763 E+02 \quad 6.772 E+03-1.372 E+03 \quad 7.035 E+01$

$-3.457 E+02 \quad 2.591 E+03 \quad 1.242 E+03-3.481 E+02$

$3.524 E+02-3.194 E+03 \quad 1.857 E+04 \quad 1.224 E+04$

$-4.625 E+02 \quad 1.067 E+04 \quad 8.396 E+03-2.275 E+03$

$5.234 E+00 \quad 7.494 E+02 \quad 5.033 E+04-4.276 E+04$

$-2.207 E+00 \quad 2.653 E+62 \quad 6.269 E+05-3.163 E+06$

$1.101 E+\emptyset 0-4.280 E+102 \quad 6.664 E+05-3.481 E+08$

3. $467 E-11 \quad 4.515 E+02$

$3.525 E+05 \quad 2.783 E+07$

$4.850 E+\varnothing 3 \oslash$.

0.

$\begin{array}{lll}7.854 E+\varnothing 2-6.513 E+81 & 2.134 E+001.138 E-01\end{array}$

$-2.484 E+03 \quad 8.950 E+83-2.019 E+03 \quad 1.171 E+02$

$-3.209 E+02 \quad 2.818 E+03 \quad 1.344 E+03-3.984 E+02$

$2.746 E+02-2.830 E+03 \quad 2.149 E+04 \quad 7.655 E+83$

$-4.181 E+\varnothing 2 \quad 1.025 E+04 \quad 1.159 E+04-3.199 E+93$

$-4.367 \mathrm{E}+01 \quad 1.966 \mathrm{E}+03 \quad 4.836 \mathrm{E}+04-3.788 \mathrm{E}+04$

$-5.177 E-01-2.546 E+01 \quad 6.941 E+95-3.733 E+96$

$1.298 E+00-5.192 E+02 \quad 7.384 E+05-5.043 E+06$

$3.890 E-\emptyset 1 \quad 4.950 E+82$

$4.111 E+65 \quad 1.855 E+27$
$Y$
$Y$
$Y$
$N$
$N$
$N$
$N$
$N$

$$
\begin{aligned}
& Y \\
& N \\
& N \\
& N \\
& N
\end{aligned}
$$

N 


\begin{tabular}{|c|c|c|c|c|c|c|c|c|}
\hline $\begin{array}{r}1 \\
1 \\
2 \\
3 \\
4 \\
5 \\
6 \\
7 \\
8 \\
9 \\
19\end{array}$ & $\begin{array}{c}\text { YTTRIUM Z/ } \\
\text { INT IDENT } \\
.01--.0232 \\
.0232--.1677 \\
1677--.8 \\
88--L 3 \\
\text { L3--L2 } \\
\text { L2--L1 } \\
\text { LI--K } \\
K--100 . \\
100 .--500 . \\
500 .--I N F\end{array}$ & $\begin{array}{l}=4.387 \\
\text { START } \\
.01 \\
.0232 \\
.1677 \\
.8 \\
2.079 \\
2.155 \\
2.373 \\
17.038 \\
100 . \\
500 .\end{array}$ & $\begin{array}{l}\text { FINISH } \\
.8232 \\
.1677 \\
.8 \\
2.079 \\
2.155 \\
2.373 \\
17.038 \\
100 . \\
500 . \\
\text { INF }\end{array}$ & $\begin{array}{l}\text { RMS } \\
8.6-2 \\
9.7-2 \\
9.2-2 \\
1.2-2 \\
6.5-9 \\
9 \cdot 1-7 \\
6.9-3 \\
6.2-3 \\
5.8-3\end{array}$ & 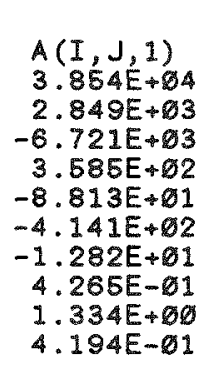 & $\begin{array}{r}A(I, J, 2) \\
-1.366 E+83 \\
-2.828 E+\emptyset 2 \\
1.298 E+04 \\
-4.223 E+02 \\
1.184 E+\emptyset 3 \\
1.043 E+\varnothing 4 \\
1.204 E+\varnothing 3 \\
-1.846 E+02 \\
-4.917 E+\varnothing 2 \\
5.651 E+\varnothing 2\end{array}$ & $\begin{array}{l}A(I, J, 3) \\
1.672 E+\emptyset 1 \\
1.529 E+\emptyset 1 \\
-3.111 E+\emptyset 3 \\
6.620 E+\emptyset 3 \\
2.198 E+04 \\
1.385 E+\emptyset 4 \\
8.028 E+04 \\
7.684 E+05 \\
8.066 E+\emptyset 5 \\
4.353 E+\emptyset 5\end{array}$ & $\begin{array}{r}A(I, J, 4) \\
-6.795 E-\emptyset 2 \\
-1.723 E-\emptyset 1 \\
1.985 E+\emptyset 2 \\
-2.796 E+\emptyset 3 \\
-1.44 \varnothing E+\emptyset 3 \\
-4.020 E+\emptyset 3 \\
-5.752 E+\emptyset 4 \\
-4.421 E+\emptyset 6 \\
-5.879 E+\oslash 6 \\
2.981 E+\emptyset 7\end{array}$ \\
\hline $\begin{array}{l}40 \\
1 \\
2 \\
3 \\
4 \\
5 \\
6 \\
7 \\
8 \\
9\end{array}$ & $\begin{array}{c}\text { ZIRCONIUM } \\
.81--M 5 \\
M 5--.5 \\
.5--L 3 \\
\text { L3--L2 } \\
\text { L2--L1 } \\
\text { LI--K } \\
K--100 . \\
100 .--500 . \\
500 .--I N F\end{array}$ & $\begin{array}{l}/ A=4 . \\
.01 \\
.18 \\
.5 \\
2.223 \\
2.307 \\
2.533 \\
17.998 \\
109 . \\
500 .\end{array}$ & $\begin{array}{l}.385 E-01 \\
.18 \\
.5 \\
2.223 \\
2.307 \\
2.533 \\
17.998 \\
100 . \\
500 . \\
\text { INF }\end{array}$ & $\begin{array}{l}2.6-1 \\
1.1-1 \\
1.3-1 \\
1.7-8 \\
8.1-7 \\
3.5-3 \\
4.6-3 \\
4.4-3 \\
2.8-2\end{array}$ & 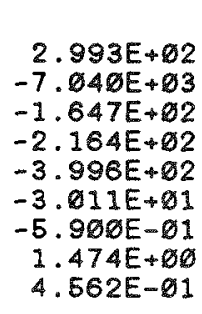 & $\begin{array}{r}1.449 E+\varnothing 2 \\
1.434 E+\varnothing 4 \\
2.296 E+\emptyset 3 \\
3.512 E+\varnothing 3 \\
1.064 E+\emptyset 4 \\
1.794 E+\varnothing 3 \\
1.945 E+\varnothing 2 \\
-5.530 E+\varnothing 2 \\
6.248 E+02\end{array}$ & $\begin{array}{r}-3.012 E+\emptyset 0 \\
-3.926 E+\emptyset 3 \\
3.166 E+\emptyset 3 \\
2.053 E+04 \\
1.548 E+04 \\
6.153 E+\emptyset 4 \\
8.031 E+\emptyset 5 \\
8.907 E+05 \\
4.760 E+\emptyset 5\end{array}$ & $\begin{array}{r}1.713 E-02 \\
2.887 E+\emptyset 2 \\
-1.127 E+\emptyset 3 \\
-3.382 E+\emptyset 3 \\
-4.776 E+\emptyset 3 \\
-5.699 E+\emptyset 4 \\
-4.579 E+\varnothing 6 \\
-7.927 E+\emptyset 6 \\
3.216 E+\emptyset 7\end{array}$ \\
\hline $\begin{array}{r}1 \\
2 \\
3 \\
4 \\
5 \\
6 \\
7 \\
8 \\
9 \\
10\end{array}$ & $\begin{array}{c}\text { NIOBIUM } \\
.01--.0297 \\
.0297--.2083 \\
.2083--.9 \\
.9--L 3 \\
\text { L3--L2 } \\
\text { L2--L1 } \\
\text { L1--K } \\
K--100 . \\
100 .--500 . \\
500 .--I N F\end{array}$ & $\begin{array}{l}=4.41 \\
.01 \\
.0297 \\
.2083 \\
.9 \\
2.370 \\
2.464 \\
2.698 \\
18.986 \\
100 . \\
500 .\end{array}$ & $\begin{array}{l}3 E-91 \\
.0297 \\
.2083 \\
.9 \\
2.370 \\
2.464 \\
2.698 \\
18.986 \\
100 . \\
500 . \\
\text { INF }\end{array}$ & $\begin{array}{l}1.4-1 \\
3 \cdot 0-2 \\
1.0-1 \\
3.1-2 \\
2.6-8 \\
7.2-7 \\
1.0-2 \\
3 \cdot 8-3 \\
4.6-3 \\
2.2-1\end{array}$ & $\begin{array}{r}3.25 \\
2.78 \\
-2.0 \\
2.15 \\
-2.98 \\
-3.98 \\
-1.65 \\
-2.25 \\
1.55 \\
4.98\end{array}$ & $\begin{array}{r}-6.844 E+\emptyset 1 \\
-4.897 E+\emptyset 2 \\
8.543 E+\emptyset 3 \\
4.490 E+\emptyset 2 \\
6.188 E+\emptyset 3 \\
1.102 E+\emptyset 4 \\
1.435 E+\emptyset 3 \\
5.516 E+\varnothing 2 \\
-5.764 E+\emptyset 2 \\
7.224 E+\emptyset 2\end{array}$ & $\begin{array}{l}75 E+00 \\
37 E+01 \\
69 E+03 \\
99 E+03 \\
35 E+04 \\
51 E+04 \\
50 E+04 \\
55 E+05 \\
43 E+05 \\
10 E+05\end{array}$ & $\begin{array}{r}-1.094 E-\emptyset 2 \\
-8.372 E-01 \\
2.770 E+\varnothing 1 \\
-2.429 E+\varnothing 3 \\
-4.420 E+\varnothing 3 \\
-5.733 E+83 \\
-7.037 E+\varnothing 4 \\
-5.018 E+\varnothing 6 \\
-8.517 E+\varnothing 6 \\
4.104 E+\varnothing 7\end{array}$ \\
\hline $\begin{array}{l}42 \\
1 \\
2 \\
3 \\
4 \\
5 \\
6 \\
7 \\
8 \\
9\end{array}$ & $\begin{array}{c}\text { MOLYBDENUM } \\
.01--.03 \\
.63--M 5 \\
M 5--.8 \\
.8--L 3 \\
\text { L3--L2 } \\
L 2--L 1 \\
\text { L1--K } \\
K--100 . \\
100 .--500 . \\
500 .--I N F\end{array}$ & $\begin{array}{l}Z / A= \\
.01 \\
.03 \\
.229 \\
.8 \\
2.521 \\
2.625 \\
2.867 \\
20 . \\
100 . \\
500 .\end{array}$ & $\begin{array}{l}4.378 \mathrm{E}-0 \\
.03 \\
.229 \\
.8 \\
2.521 \\
2.625 \\
2.867 \\
20 . \\
106 . \\
500 . \\
\text { INF }\end{array}$ & $\begin{array}{l}2.2-1 \\
6.2-2 \\
9.0-2 \\
3 . \emptyset-2 \\
3 . \emptyset-8 \\
6.5-7 \\
6.2-3 \\
3.6-3 \\
3.1-3 \\
2.3-2\end{array}$ & $\begin{array}{r}-3.508 E+03 \\
3.653 E+03 \\
-7.786 E+03 \\
-1.114 E+02 \\
-3.027 E+02 \\
-3.784 E+02 \\
-1.840 E+01 \\
-2.560 E+00 \\
1.540 E+\varnothing 0 \\
5.359 E-01\end{array}$ & $\begin{array}{r}3.050 E+02 \\
-6.192 E+\emptyset 2 \\
1.722 E+\emptyset 4 \\
1.895 E+\emptyset 3 \\
7.576 E+\emptyset 3 \\
1.099 E+\varnothing 4 \\
1.552 E+03 \\
7.317 E+\emptyset 2 \\
-4.834 E+\emptyset 2 \\
7.662 E+\varnothing 2\end{array}$ & 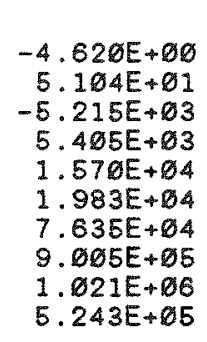 & $\begin{array}{r}1.998 E-02 \\
-9.955 E-01 \\
3.992 E+02 \\
-2.261 E+\square 3 \\
-4.795 E+\$ 3 \\
-6.801 E+\varnothing 3 \\
-8.181 E+04 \\
-5.349 E+06 \\
-9.300 E+86 \\
5.200 E+\varnothing 7\end{array}$ \\
\hline $\begin{array}{r}43 \\
1 \\
2 \\
3 \\
4 \\
5 \\
6 \\
7 \\
8 \\
9\end{array}$ & $\begin{array}{c}\text { TECHNETIUM } \\
.01--.068 \\
.068--M 5 \\
M 5--M 3 \\
M 3--L 3 \\
L 3--L 2 \\
\text { L2--L1 } \\
\text { LI--K } \\
K--100 . \\
100 .--500 . \\
500 .--I N F\end{array}$ & $\begin{array}{l}Z / A= \\
.01 \\
.068 \\
.253 \\
.425 \\
2.677 \\
2.793 \\
3.043 \\
21.044 \\
100 . \\
500 .\end{array}$ & $\begin{array}{l}4.388 E-0 \\
.068 \\
.253 \\
.425 \\
2.677 \\
2.793 \\
3.043 \\
21.044 \\
100 . \\
500 . \\
\text { INF }\end{array}$ & $\begin{array}{c}N A \\
9.3-2 \\
2.4-2 \\
3.6-2 \\
3.7-8 \\
1.3-7 \\
4.1-3 \\
5.8-3 \\
4.5-3 \\
2.4-2\end{array}$ & $\begin{array}{r}2.680 E \\
-7.592 E \\
-3.269 E \\
-3.863 E \\
-2.871 E \\
-1.101 E \\
-2.936 E \\
5.721 E \\
1.769 E \\
5.748 E\end{array}$ & $\begin{array}{r}0 . \\
8.677 E+\varnothing 2 \\
4.110 E+\emptyset 4 \\
3.458 E+\emptyset 3 \\
7.198 E+\emptyset 3 \\
1.635 E+\emptyset 4 \\
1.924 E+03 \\
7.164 E+\varnothing 1 \\
-5.691 E+\emptyset 2 \\
8.119 E+\emptyset 2\end{array}$ & $\begin{array}{r}0 . \\
-1 . \\
-1 . \\
3 . \\
1 . \\
3 . \\
7 . \\
1 . \\
1 . \\
6 .\end{array}$ & $\begin{array}{r}0 . \\
6.198 E+\varnothing 0 \\
1.384 E+\varnothing 3 \\
-1.341 E+\varnothing 3 \\
-5.767 E+\varnothing 3 \\
-1.491 E+\varnothing 3 \\
-8.669 E+\varnothing 4 \\
-6.678 E+\varnothing 6 \\
-1.069 E+\varnothing 7 \\
3.605 E+07\end{array}$ \\
\hline $\begin{array}{r}44 \\
1 \\
2 \\
3 \\
4 \\
5 \\
6 \\
7 \\
8 \\
9\end{array}$ & $\begin{array}{c}\text { RUTHENIUM } \\
\text {.01--. } 716 \\
.0716--M 5 \\
M 5--M 3 \\
M 3--L 3 \\
L 3--L 2 \\
L 2--L 1 \\
L 1--K \\
K--100 . \\
100 .--500 . \\
500 .--I N F\end{array}$ & $\begin{array}{l}Z / A=4 \\
.01 \\
.0716 \\
.2794 \\
.4606 \\
2.838 \\
2.967 \\
3.224 \\
22.117 \\
100 . \\
500 .\end{array}$ & $\begin{array}{l}.353 E-01 \\
.0716 \\
.2794 \\
.4606 \\
2.838 \\
2.967 \\
3.224 \\
22.117 \\
106 . \\
5 ø \emptyset . \\
\text { INF }\end{array}$ & $\begin{array}{l}1 \cdot 5-1 \\
8 \cdot 2-2 \\
2 \cdot 0-2 \\
3 \cdot 5-2 \\
4 \cdot 4-8 \\
5 \cdot 3-7 \\
5 \cdot 1-3 \\
6.2-3 \\
1.2-2 \\
2 \cdot 5-2\end{array}$ & $\begin{array}{r}2.116 \\
1.947 \\
-2.141 \\
-2.430 \\
-2.831 \\
-3.547 \\
-1.311 \\
1.007 \\
1.633 \\
6.218\end{array}$ & $\begin{array}{r}1.08 \\
7.27 \\
2.87 \\
2.78 \\
7.88 \\
1.09 \\
1.42 \\
1.27 \\
-4.03 \\
9.11\end{array}$ & $\begin{array}{r}-2.614 \\
-1.490 \\
-8.592 \\
5.237 \\
1.913 \\
2.575 \\
9.237 \\
1.086 \\
1.144 \\
5.873\end{array}$ & $\begin{array}{r}1.466 E-02 \\
8.002 E+00 \\
8.312 E+02 \\
-2.042 E+03 \\
-6.518 E+03 \\
-9.586 E+103 \\
-1.148 E+05 \\
-7.755 E+06 \\
-9.817 E+06 \\
6.614 E+\varnothing 7\end{array}$ \\
\hline
\end{tabular}


45 RHODIUM

\begin{tabular}{ccc} 
RODIUM & Z/A & \multicolumn{3}{c}{$4.373 E-\$ 1$} \\
INT IDENT & START & FINISH \\
$.61--.072$ & .01 & .072 \\
$.072--M 5$ & .072 & .307 \\
$M 5--M 3$ & .307 & .4982 \\
$M 3--L 3$ & .4962 & 3.004 \\
L3--L2 & 3.094 & 3.146 \\
L2--LI & 3.146 & 3.412 \\
LI--K & 3.412 & 23.220 \\
$K--100$. & 23.220 & 100. \\
$100 .-500$. & 100. & 500. \\
500.--INF & 500. & INF
\end{tabular}

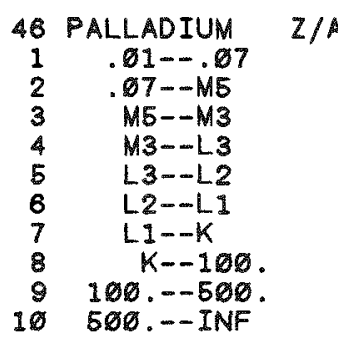

RMS

2.1-1

7.2-2

1. 3-2

3. 8-2

5. $2-8$

4. 9-7

4. 3-3

2. $9-3$

3. 8-3

2. 4-2

2.3-1

5. 3-1

2. 5-2

4. 1-2

6. $5-8$

4. 3-7

8. $4-3$

3.9-3

2. 6-3

2. 2-2

1. $0-1$

1. 2-1

4.8-7

4.1-2

$7.3-8$

4. $0-7$

4.1-3

3. 3-3

2.7-3

2. 3-2

$100 .-100$

$500 .--$ INF

500. INF

$\begin{array}{rccc}48 & \text { CADMIUM } & Z / A=4.270 E-\varnothing 1 \\ 1 & .01--.03 & .01 & .03 \\ 2 & .03--.998 & .03 & .098 \\ 3 & .998--M 5 & .098 & .404 \\ 4 & M 5--M 3 & .404 & .6165 \\ 5 & M 3-L 3 & .6165 & 3.537 \\ 8 & L 3--L 2 & 3.537 & 3.727 \\ 7 & L 2-L 1 & 3.727 & 4.018 \\ 8 & \text { LI--K } & 4.018 & 26.711 \\ 9 & K--100 . & 26.711 & 100 . \\ 10 & 100 .--500 . & 109 . & 500 . \\ 11 & 500 .--I N F & 500 . & \text { INF }\end{array}$

49 INDIUM $Z / A=4.268 E-01$

\begin{tabular}{|c|c|c|c|}
\hline $\begin{array}{c}.01--.031 \\
.031--.103 \\
193--M 5 \\
M 5--M 2 \\
M 2--L 3 \\
L 3-L 2 \\
L 2--L 1 \\
L 1--K \\
K--100 . \\
100 .--500 . \\
500 .--I N F\end{array}$ & $\begin{array}{l}.01 \\
.031 \\
.103 \\
.443 \\
.702 \\
3.730 \\
3.938 \\
4.238 \\
27.94 \\
100 . \\
500 .\end{array}$ & $\begin{array}{l}.831 \\
.103 \\
.443 \\
.762 \\
3.730 \\
3.938 \\
4.238 \\
27.94 \\
106 . \\
506 . \\
\text { INF }\end{array}$ & $\begin{array}{l}\text { NA } \\
1.3-1 \\
2.6+D \\
9.6-3 \\
5.7-2 \\
9.8-8 \\
3.2-7 \\
2.9-3 \\
4.1-3 \\
5.5-3 \\
2.4-2\end{array}$ \\
\hline $\begin{array}{c}\text { TIN } Z / A= \\
.01--.9318 \\
.0318--.093 \\
.093--M 5 \\
M 5--M 3 \\
M 3--L 3 \\
L 3--L 2 \\
L 2--L 1 \\
L 1--K \\
K--100 . \\
190 .--509 . \\
500 .--I N F\end{array}$ & $\begin{array}{l}4.213 E-\emptyset 1 \\
.01 \\
.0318 \\
.093 \\
.485 \\
.714 \\
3.929 \\
4.156 \\
4.485 \\
29.2 \\
100 . \\
500 .\end{array}$ & $\begin{array}{l}.0318 \\
.093 \\
.485 \\
.714 \\
3.929 \\
4.156 \\
4.465 \\
29.2 \\
100 . \\
500 . \\
\text { INF }\end{array}$ & $\begin{array}{c}\text { NA } \\
5.6-2 \\
2.6+\varnothing \\
8.5-3 \\
8.7-2 \\
1.1-7 \\
3.0-7 \\
1.1-2 \\
3.0-3 \\
2.2-3 \\
2.0-2\end{array}$ \\
\hline
\end{tabular}

NA

6.1-2

$2.6+\infty$

$9.7-3$

$8 \cdot 5-2$

$8.5-8$

$3.4-7$

$3.4-3$

$2.6-3$

$9.7-3$

$2.4-2$

\begin{tabular}{|c|c|c|c|}
\hline $\begin{array}{l}A(I, J, 1) \\
2.535 E+03 \\
1.037 E+03 \\
1.155 E+\varnothing 3 \\
4.773 E+\emptyset 2 \\
2.865 E+\varnothing 2 \\
3.285 E+\varnothing 2 \\
2.532 E+\varnothing 1 \\
3.043 E+\varnothing 0 \\
1.870 E+\varnothing 6\end{array}$ & $\begin{array}{l}A(I, J, 2) \\
1.329 E+02 \\
1.357 E+03 \\
3.456 E+03 \\
3.678 E+03 \\
7.197 E+03 \\
9.342 E+03 \\
2.099 E+03 \\
9.079 E+02 \\
-4.579 E+02 \\
9.644 E+02\end{array}$ & $\begin{array}{r}A(I, J, 3) \\
-3.193 E+00 \\
-2.412 E+02 \\
8.529 E+\emptyset 2 \\
5.261 E+\varnothing 3 \\
2.392 E+04 \\
3.464 E+64 \\
9.447 E+04 \\
1.128 E+06 \\
1.251 E+68 \\
7.281 E+\varnothing 5\end{array}$ & $\begin{array}{r}A(I, J, 4) \\
1.848 E-\emptyset 2 \\
1.212 E+\emptyset 1 \\
-3.075 E+\emptyset 2 \\
-2.225 E+\varnothing 3 \\
-7.946 E+\varnothing 3 \\
-1.203 E+04 \\
-1.115 E+\emptyset 5 \\
-8.088 E+\varnothing 6 \\
-1.191 E+07 \\
4.409 E+\varnothing 7\end{array}$ \\
\hline
\end{tabular}

$1.095 E+15-2.552 E+02 \quad 1.914 E+90-3.918 E-03$

$2.418 E+\$ 3 \quad 9.664 E+01-1.850 E+D 2 \quad 9.047 E+06$ $-1.605 E+04 \quad 3.167 E+04-1.240 E+041.658 E+03$ $-4.705 E+82 \quad 3.846 E+03 \quad 5.959 E+03-2.839 E+83$

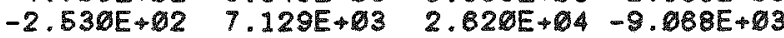
$-3.225 E+02 \quad 1.01 E+04 \quad 3.550 E+04-1.354 E+84$ $1.642 E+D 1 \quad 4.683 E+02 \quad 1.181 E+D 5-1.737 E+05$ $-2.888 E+00 \quad 8.714 E+02 \quad 1.203 E+\varnothing 6-9.396 E+86$ $1.857 E+00-3.862 E+02 \quad 1.328 E+86-1.219 E+07$ $\begin{array}{llll}7.151 E-01 \quad 1.066 E+03 & 7.318 E+05 & 0.061 E+07\end{array}$

$1.054 E+04-1.973 E+02-1.033 E+00-8.574 E-04$ $-4.089 E+03 \quad 3.916 E+63-8.605 E+02 \quad 4.841 E+01$ $-2.951 E+63 \quad 8.256 E+83 \quad 1.909 E+13-8.620 E+02$ $-2.321 E+91 \quad 1.892 E+03 \quad 9.451 E+03-4.150 E+03$ $-2.547 E+62 \quad 7.851 E+03 \quad 2.733 E+04-1.638 E+04$ $-3.170 E+02 \quad 1.045 E+04 \quad 3.850 E+04-1.560 E+04$ $-1.817 E+D 0 \quad 1.285 E+\varnothing 3 \quad 1.192 E+\varnothing 5-1.684 E+85$ $-4.061 E+90 \quad 1.339 E+03 \quad 1.260 E+06-9.752 E+86$ $2.065 E+06-4.444 E+62 \quad 1.433 E+08-1.533 E+87$ $7.734 E-01 \quad 1.157 E+03$

$8.066 \mathrm{E}+05 \quad 8.214 \mathrm{E}+67$

0.

$1.876 E+93$

$4.833 E+02 \quad 1.167 E+03$

$2.088 E+13 \quad 3.038 E+163$

$7.508 E+002.031 E+103$

$-2.454 E+\square 2 \quad 8.182 E+03$

$-2.677 E+62 \quad 8.244 E+63$

$-2.308 E+01 \quad 2.129 E+03$

$-8.208 E+\emptyset 0 \quad 2.269 E+\emptyset 3$

$2.254 E+00-4.904 E+102$

$8.123 E-D 1 \quad 1.229 E+\square 3$

$1.702 E+630$.

2.333E+ $1.51 .516 E+03$

$-4.354 E+03 \quad 4.933 E+83$

$-2.048 E+103$

$-1.763 E+02$

$-2.324 E+102$

$-2.675 E+02$

$-2.149 E+01$

$-2.097 E+00$

$2.233 E+00$

$9.964 E+103$

$3.053 E+03$

$7.725 E+03$

$8.828 E+103$

$2.143 E+103$

$1.045 E+13$

8.

1. $331 E+103$

$1.857 E+03$

$6.119 E+111.789 E+63-5.287 E+02 \quad 4.438 E+21$

$2.325 E+03 \quad 2.985 E+03 \quad 1.395 E+03-1.168 E+52$

$-8.411 E+62 \quad 7.174 E+63$

$-2.078 E+12 \quad 6.795 E+83$

$-2.735 \mathrm{E}+102 \quad 1.006 \mathrm{E}+\emptyset 4$

1. $409 E+\varnothing 1 \quad 5.245 E+\varnothing 2$

$-6.053 E+902.152 E+03$

$2.434 E+\varnothing 0-4.441 E+82$

$9.158 E-61 \quad 1.438 E+83$
.

$3.498 E+81-2.775 E-1$ $-1.221 E+\$ 3 \quad 9.073 E+81$ $-2.857 E+83 \quad 7.223 E+12$ $3.333 E+84-1.361 E+04$ $5.249 E+04-2.065 E+04$ $1.258 E+65-1.775 E+05$ $1.428 E+\emptyset 6-1.216 E+\emptyset 7$ $1.578 E+86-1.747 E+07$ $9.171 E+05 \quad 6.234 E+07$ 3. $429 E+83-1.945 E+03$ $3.953 E+04-1.548 E+84$ $5.154 E+04-2.316 E+04$ $1.554 E+05-2.738 E+05$ 1. $430 E+06-1.218 E+57$ $1.894 E+06-2.116 E+07$ $9.506 E+25 \quad 6.996 E+07$ 
51 ANTIMONY

$\begin{array}{rc}51 & \text { ANTIMONY } \\ J & \text { INT IDENT } \\ 1 & .01--.0304 \\ 2 & .0384--.898 \\ 3 & .096--M 5 \\ 4 & M 5--M 3 \\ 5 & M 3--L 3 \\ 6 & L 3--L 2 \\ 7 & L 2--L 1 \\ 8 & L 1--K \\ 9 & K--100 . \\ 10 & 108 .-500 . \\ 11 & 500 .--I N F\end{array}$

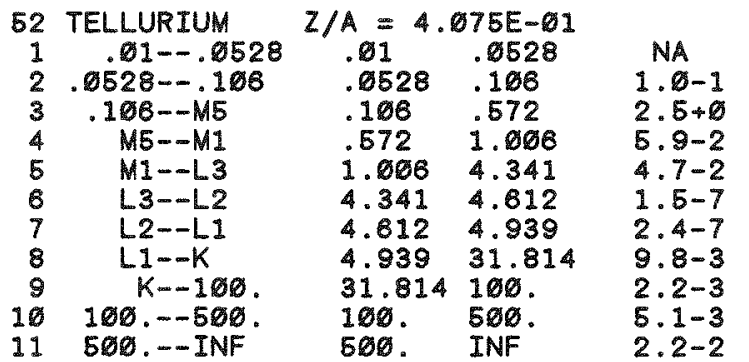

53 IODINE $Z / A=4.176 E-01$

NA

$\frac{1}{2 \cdot 5-1}$

$5.9-2$

$4.7-2$
$1.5-7$

$.4-7$

$2-3$

$2.2-2$

NA

1. 8-1

1. $4+0$

9.7-3

9.5-2

1. $8-1$

1. $6-7$

2. $1-7$

3. 5-3

2. $9-3$

4. $7-3$

2. 3-2
$/ A=4.189 E-01$ START FINISH $.01 \quad .0304$ $.096 \quad .528$ $.528 \quad .766$ $.766 \quad 4.132$ $4.132 \quad 4.381$ $4.381 \quad 4.698$ $4.698 \quad 36.491$ 30.491100 100.500 59פ. INF

$\begin{array}{ll}4.176 E-101 \\ .01 & .034 \\ .034 & .103 \\ .193 & .819 \\ .819 & .931 \\ .931 & 1.072 \\ 1.072 & 4.557 \\ 4.557 & 4.852 \\ 4.852 & 5.188 \\ 5.188 & 33.17 \\ 33.17 & 106 . \\ 106 . & 500 . \\ 500 . & \text { INF }\end{array}$

RMS
NA
$4 \cdot 9-2$
$2 \cdot 6-1$
$8 \cdot 7-3$
$8 \cdot 4-2$
$1 \cdot 3-7$
$2 \cdot 5-7$
$2 \cdot 8-3$
$8 \cdot 7-4$
$8 \cdot 7-3$
$2 \cdot 3-2$

NA

$4 \cdot 9-2$

$8.7-3$

1. $3-7$

. $5-7$

$7-4$

$7-4$
$2.3-2$

NA

1. 8-1

9.2-1

4. 6-2

5. -2

1.9-2

1. 7-7

1. 9-7

5. 1-3

2. $2-3$

5. 1-3

2.1-2

100. 500.

\section{6}$$
\frac{2}{3}
$$$$
5
$$$$
5
$$$$
6
$$$$
\begin{aligned}
& 7 \\
& 8 \\
& 9
\end{aligned}
$$$$
10
$$

$10100 .-500$
$11500 .-1 \mathrm{NF}$ $Z / A=4.138 E-\emptyset 1$ $.01-.0704 \quad .01 \quad .0704$

$.0704-.111 \quad .0704 \quad .111$

$.111--M 5$

M5 - - M2

M2- $-L 3$

$L 3--L 2$

L2--LI

$L 1--K$

K-- 190

.111

.726

$\begin{array}{ll}126 & 1.065\end{array}$

$1.065 \quad 5.012$

$5.012 \quad 5.36$

$5.36 \quad 5.713$

$\begin{array}{ll}5.713 & 35.9 \\ 35.985 & 109\end{array}$

100. 500 .

5ø0. INF

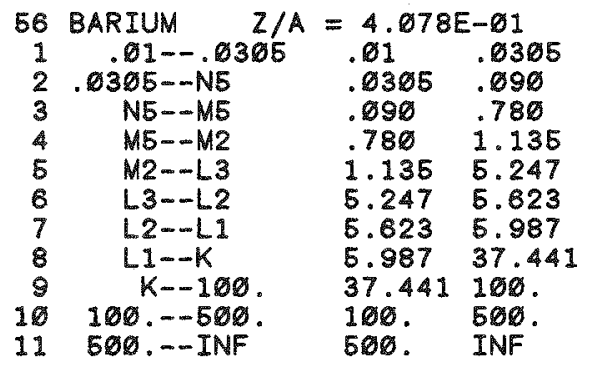

NA

5.8-2

$1.6+10$

$7.5-3$

1.6-1

1. 2-7

1. 8-7

8. 3-3

2. $5-3$

5. 6-3

$2.2-2$

NA

2. $5-2$

$1.8+6$

5. 9-2

$5.5-2$

1. $7-7$

1. 5-7

1. 3-2

2. $6-3$

4. 4-3

2. $2-2$

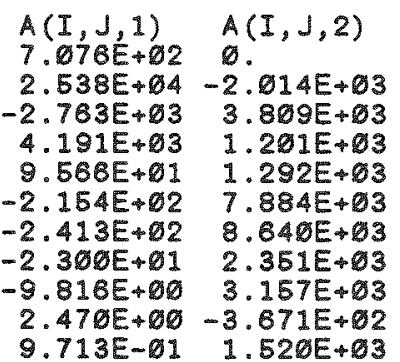

$A(I, J, 3) \quad A(I, J, 4)$

$5.518 E+01-5.093 E-\emptyset 1$

$-9.187 E+02 \quad 6.404 E+101$

$6.138 E+02 \quad 8.057 E+02$

$1.534 E+04-8.151 E+03$

$3.884 E+04-1.742 E+104$

$6.267 E+\emptyset 4-2.629 E+\square$

$1.410 E+\emptyset 5-2.13 \emptyset E+\emptyset 5$

1. $443 E+08-1.193 E+07$

$1.779 E+06-2.260 E+07$

$1.116 \mathrm{E}+1887.468 \mathrm{E}+27$

$9.416 E+02$

$-5.818 E+104$

$-2.734 E+103$

$-1.058 E+05$

$-4.397 E+11$

$-1.946 E+02$

$-2.372 E+62$

$1.086 \mathrm{E}+\varnothing \varnothing$

$-3.401 E+00$

$2.389 E+00$

1.

$\$$.

$\varnothing$. $3.947 E+03-9.985 E+02 \quad 7.313 E+01$ $2.917 E+05-2.491 E+05 \quad 7.124 E+04$ $2.059 E+63 \quad 1.571 E+14-9.021 E+D 3$ $7.129 E+13 \quad 4.399 E+4-1.939 E+04$ $9.166 E+83 \quad 6.368 E+04-2.911 E+04$ $1.149 E+03 \quad 1.640 E+05-2.950 E+05$ $1.832 E+03 \quad 1.571 E+06-1.428 E+07$

$1.798 E+\varnothing 6-2.273 E+07$ $1.076 \mathrm{E}+667.154 \mathrm{E}+97$

$7.02 D E+D 2 D$.

2. $410 E+84-2.114 E+03$

$\emptyset$.

$-2.554 E+03$

$4.115 E+B 3$

$624 E+81-6$

$-4.564 E+04$

$1.940 E+104$

$1.181 E+05$

$-1.059 E+63$

0.

$-1.016 E+\infty 3$

$-1.833 E+02$

$-2.158 E+02$

$-2.586 E+91$

$-3.670 E+90$

2. $225 E+80$

$-1.268 E+84$

. $.556 E+84$

$266-81$

1. $150 E+104$

$8.275 E+03$

$2.615 E+13$

$1.872 E+103$

$1.829 E+02$

$1.755 E+13$

$.443 E+64$

$7.800 E+01$

$2.490 E+64$

1. $681 E+04$

$5.201 E+04-2.240 E+04$

$7.525 E+04-3.297 E+104$

$1.595 E+05-2.557 E+05$

$1.719 E+96-1.646 E+07$

$1.881 E+26-2.157 E+D 7$

$1.154 E+66 \quad 8.971 E+67$

$6.262 E+02$.

$7.578 E+04-1.206 E+04$

0.

b.

$-1.456 E+03 \quad 3.607 E+03-1.050 E+03 \quad 8.562 E+01$

$-2.422 E+04 \quad 6.454 E+04-3.914 E+04 \quad 8.599 E+\infty 3$

$-6.606 E+82 \quad 7.269 E+03$

$-1.565 E+02$

$-2.236 E+\varnothing 2$

$5.749 E+03$

$9.444 E+103$

$-2.010 E+01$

$2.400 E+183$

$-1.622 E+00$

2. $282 E+00$

1. $836 \mathrm{E}+03$

$3.626 E+b 2$

$8.119 E+63-3.383 E+53$

$5.985 E+104-2.396 E+04$

$7.463 E+104-3.742 E+154$

$1.720 E+D 5-2.977 E+05$

$1.780 E+06-1.721 E+87$

$1.982 E+86-2.448 E+07$

$1.133 E+86 \quad 1.244 E+88$

$6.813 E+02$

$1.642 E+85-2.922 E+64$

$-2.490 E+\varnothing 3 \quad 4.936 E+63$

$1.295 E+84-7.423 E+83$

$8.121 E+02-1.572 E+03$

$-8.402 E+01 \quad 2.872 E+03$

$-2.254 E+82 \quad 1.015 E+24$

$-1.987 E+00 \quad 1.406 \mathrm{E}+03$

$-4.238 E+002.444 E+63$

$2.278 E+00 \quad 4.044 E+82$

1. $226 E+60$

$4.044 E+02$
$2.030 E+03$

$5.765 E+03$.

$1.759 E+03-2.331 E+02$

$-4.276 E+02 \quad 2.995 E+83$

$6.154 E+03 \quad 1.195 E+03$

$2.420 E+\varnothing 2-4.033 E+\varnothing 2$

$-1.884 E+62 \quad 4.043 E+183$

$-1.836 E+02 \quad 7.952 E+03$

$8.708 E+007.581 E+62$

$-5.846 E+00$

$2.590 \mathrm{E}+100$

1.281E+ $0 \%$
๑.

$1.724 E+13-3.361 E+\emptyset 1$ $-1.531 E+83 \quad 1.354 E+\varnothing 2$ $-6.620 E+03 \quad 9.125 E+\varnothing 3$ $2.712 E+04-1.563 E+104$ $7.699 E+04-1.853 E+04$ $7.940 E+04-4.284 E+104$ $2.048 E+05-4.166 E+\varnothing 5$ $1.873 E+06-1.899 E+07$ $2.085 E+06-2.635 E+07$ $1.249 E+\varnothing 6 \quad 1.169 E+\varnothing 8$

0.

$9.598 E+00 \quad 3.968 \mathrm{E}-02$ $-9.837 E+62 \quad 8.771 E+D 1$ $-2.858 E+02 \quad 1.593 E+103$ $3.138 E+04-2.304 E+104$ $7.718 E+04-2.532 E+104$ $9.545 E+D 4-4.532 E+104$ $2.258 E+05-5.011 E+05$ $1.982 E+06-1.948 E+87$ $2.236 E+6-3.193 E+07$ $1.303 E+06 \quad 1.193 E+68$

$U$
$Y$
$Y$
$Y$
$Y$
$Y$
$N$
$N$
$N$
$N$
$N$
$N$

$Y$
$Y$
$Y$
$Y$
$N$
$N$
$N$
$N$
$N$
$N$
$N$

$Y$
$Y$
$Y$
$Y$
$Y$
$Y$
$N$
$N$
$N$
$N$
$N$
$N$

$Y$
$Y$
$Y$
$Y$
$N$
$N$
$N$
$N$
$N$
$N$
$N$
$N$

$Y$
$Y$
$Y$
$Y$
$Y$
$N$
$N$
$N$
$N$
$N$
$N$

$Y$
$Y$
$Y$
$Y$
$Y$
$N$
$N$
$N$
$N$
$N$
$N$




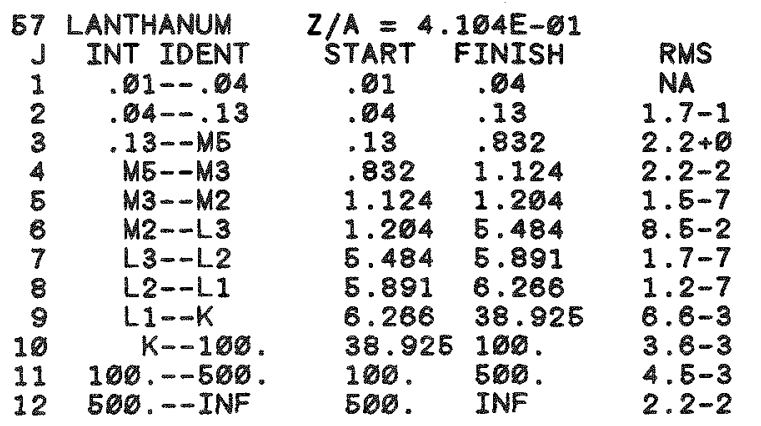

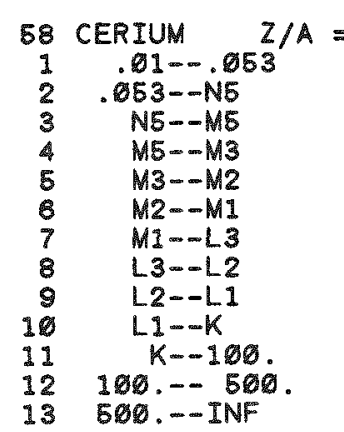
$.053 \quad .110$

\begin{tabular}{|c|c|}
\hline 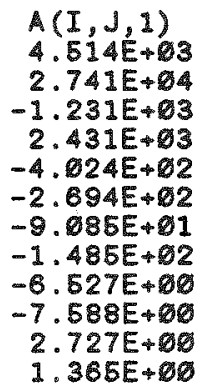 & 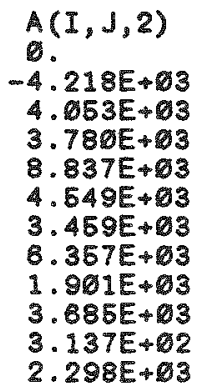 \\
\hline
\end{tabular}

$A(I, J, 3) \quad A(I, J, 4)$

0

$2.117 E+12-3.183 E+60$ $-1.329 E+1.31 .263 E+02$ $2.792 E+03-2.167 E+02$ $2.398 E+03-4.154 E+02$ $1.944 E+04-1.264 E+04$ $8.639 E+104-2.558 E+64$ $1.121 E+25-4.628 E+04$ $2.289 E+165-4.652 E+65$ $1.991 E+06-2.054 E+07$ $2.356 E+66-3.382 E+67$ $1.393 E+06 \quad 1.220 E+08$
NA $.01^{139 E-01}$ .110 .883 $.883 \quad 1.185$ $1.185 \quad 1.273$ $1.273 \quad 1.435$ $1.435 \quad 5.723$ $5.723 \quad 6.164$ 6.1846 .549 $8.549 \quad 40.443$ 46.443100

106. 520

500. INF

$2 / A=4.187 E-01$

$.01 \quad .04$ NA

$.04 \quad .113 \quad 9.1-2$

$.113 . .931$

$.931 \quad 1.242$

$1.242 \quad 1.337$

1.3371 .505

$1.505 \quad 5.964$

5.9846 .44

6.446 .835

$6.835 \quad 41.991$

41.991160 .

100 . 500

$100 .--500$

$500 .-$ INF

$3 \cdot 2-1$

$8.5-2$

$5.3-6$

$9.7-7$

5. $7-2$

1. $7-7$

$1.3-7$

$1.3-2$

$7.6-4$

. 3-3

2. 8-2

. $1-2$

$8.1-2$

$3.6-7$

1. 3-6

6. 3-2

$1.2-7$

$1.0-7$

1. $1-2$

$3.2-4$

$6.0-3$

2. 4-2

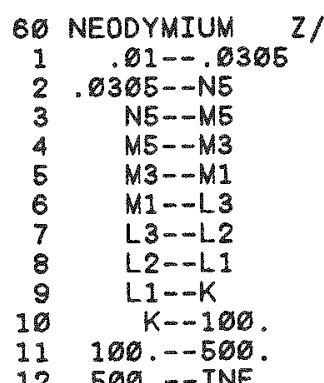

$/ A=4.168 E-D 1$

.01 .0305

$.0385 \quad .117$

$.117 \quad .978$

.9781 .298

$1.298 \quad 1.575$

$1.575 \quad 6.208$

6.2586 .722

6.7227 .128

$7.128 \quad 43.569$

43.569100

500.- - INF

100.500.

500. INF

\begin{tabular}{rccl}
61 & PROMETHIUM & \multicolumn{2}{l}{$Z / A=4.207 E-01$} \\
1 & $.01--.0305$ & .01 & .0305 \\
2 & $.0305--N 5$ & .0365 & .122 \\
3 & $N 5--M 5$ & .122 & 1.027 \\
4 & $M 5--M 3$ & 1.027 & 1.357 \\
5 & $M 3--M 2$ & 1.357 & 1.471 \\
6 & $M 2--M 1$ & 1.471 & 1.648 \\
7 & $M 1--L 3$ & 1.648 & 6.459 \\
8 & $L 3-L 2$ & 6.459 & 7.013 \\
9 & $L 2--L 1$ & 7.013 & 7.428 \\
10 & $L 1--K$ & 7.428 & 45.184 \\
11 & $K--100$. & 45.184 & 109. \\
12 & $100 .--500$. & 100. & 501. \\
13 & $500 .--I N F$ & 500. & $I N F$
\end{tabular}

NA

8.1-2

$1.5+8$

$8.4-2$

$4.3-6$

6.8-2

$2 \cdot 1-7$

8. $2-8$

$7.6-3$

$2,1-4$

$6.5-4$

$2 \cdot 3-2$

NA

$1.2-1$

$1.2+8$

$4.2-5$

$4.2-7$

1. $5-6$

$2 \cdot 2-3$

$2 \cdot 6-7$

$3.6-7$

$8.2-3$

$9.7-4$

$5.3-3$

$2.4-2$
$1.542 E+03$

$2.448 E+84-5.012 E+83$

$-1.622 E+03 \quad 4.662 E+03$

$5.551 E+04-1.789 E+95$

$4.656 E+63 \quad 8.680 E+63$

$-2.828 E+62 \quad$ I. $019 E+04$

$3.264 E+02-7.446 E+152$

$-7.263 E+01 \quad 2.822 E+83$

$-1.695 E+82$

$1.939 E+01$

$-1.029 E+01$

$2.723 E+80$

1. $464 E+06$

$7.899 E+93$

$3.799 E+182$

4. $489 E+03$

4. $916 E+82$

$2.444 E+63$

$3.935 E+93$

$5.885 E+81$

$-2.475 E+63$

$-6.489 E+63$

$-5.349 E+62$

$-3.829 E+122$

1. $158 E+02$

$-4.523 E+61$

$-1.401 E+12$

$8.492 E+106$

$-9.126 E+10$

$3.207 E+00$

1.557E+D0

2. $2.485 E+64-1.066 E+242.589 E+03$ $8.049 E+63 \quad 5.499 E+83-9.313 E+92$

$1.003 E+04 \quad 3.437 E+03-7.202 E+02$ $1.754 E+03 \quad 2.978 E+04-2.081 E+04$ $1.773 E+03 \quad 1.075 E+05-1.884 E+04$ $6.504 E+03 \quad 1.315 E+25-5.758 E+04$ $8.884 E+22 \quad 2.819 E+85-7.256 E+65$ $4.223 E+03 \quad 2.298 E+66-2.709 E+07$ $3.424 E+02 \quad 2.697 E+86-4.123 E+07$ $2.710 E+13$

1. $568 E+98 \quad 1.383 E+98$

$2.269 E+03$

$-1.142 E+D 3$

$-4.138 E \rightarrow 02$

1. $241 E+104$

2. $239 E+102$

$-3.536 E+02$

$-6.644 E+D 1$

$-1.114 E+12$

8.287E+DE

$-9.944 E+00$

$3.820 E+D 0$

$1.633 E+00$

8.

$4.035 E+02-1.726 E+1$

$3.657 E+03-1.285 E+03$

$-6.608 E+03-8.545 E+83$

$1.199 E+04-1.181 E+23$

$2.479 E-81$

$1.287 \mathrm{E}+82$

$1.174 E+64$

$6.609 E+03 \quad 1.641 E+04-7.462 E+93$

$2.796 E+03 \quad 1.098 E+05-2.924 E+04$

$5.266 E+03 \quad 1.457 E+95-5.559 E+04$

$9.606 E+02 \quad 2.935 E+05-7.637 E+65$

$4.886 E+03 \quad 2.320 E+06-2.702 E+87$

$8.492 E+01 \quad 2.868 E+06-4.750 E+07$

$2.833 E+83$

$1.759 E+66 \quad 9.295 E+27$

$2.285 E-13$

$3.175 E+23$

$-7.108 E+102$

$-5.546 E+102$

$-5.157 E+82$

$-4.697 E \rightarrow 82$

$-1.912 E+22$

$-7.165 E+81$

$-2.589 E+62$

$-4.251 E+01$

$9.003 E-02$

$2.857 E+\varnothing 0$

1. $742 E+\infty$
$4.311 E+02$

$7.102 E+03$

$8.937 \mathrm{E}+183$

$9.760 E+83$

$5.138 E+63$

$3.169 E+83$

$8.215 E+163$

$4.740 E+\varnothing 3$

$2.739 E+83$

1. $090 \mathrm{E}+03$

$3.089 E+03$ c.

$2.911 E+21-5.124 E-61$ $-1.397 E+63 \quad 1.482 E+82$ $4.958 E+53-7.806 E+102$ $6.068 E+13-1.148 E+103$ $6.028 E+03-1.298 E+03$ $2.120 E+04-1.345 E+04$ $1.186 E+85-3.499 E+64$ $-1.162 E+165 \quad 1.824 E+106$ $2.377 E+85-4.279 E+85$ $2.841 E+56-3.338 E+87$ $2.888 E+86-4.432 E+07$ $1.744 E+06 \quad 1.719 E+08$

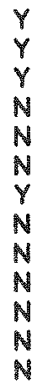

$N$ 


\begin{tabular}{|c|c|c|c|c|c|c|c|c|}
\hline $\begin{array}{r}62 \\
j \\
1 \\
2 \\
3 \\
4 \\
5 \\
8 \\
7 \\
8 \\
9 \\
12\end{array}$ & $\begin{array}{c}\text { SAMARIUM } \\
\text { INT IDENT } \\
.01=-.0305 \\
.0305=-N 5 \\
\text { NE- }-M 5 \\
M 5--M 3 \\
M 3--L 3 \\
L 3--L 2 \\
L 2--L 1 \\
L 1--K \\
K--100 . \\
106 .--500 . \\
560 .=-I N F\end{array}$ & $\begin{array}{l}\text { A. }=4.1 \\
\text { START } \\
.61 \\
.0305 \\
.128 \\
1.078 \\
1.419 \\
8.716 \\
7.312 \\
7.736 \\
48.834 \\
106 . \\
500 .\end{array}$ & $\begin{array}{l}23 E-\varnothing 1 \\
F I N I S H \\
.0305 \\
.128 \\
1.878 \\
1.419 \\
6.716 \\
7.312 \\
7.736 \\
48.834 \\
109 . \\
500 . \\
\text { INF }\end{array}$ & $\begin{array}{c}\text { RMS } \\
\text { NA } \\
5.1-2 \\
2.3+8 \\
4.0-5 \\
1.8-1 \\
1.6-7 \\
4.4-8 \\
7.7-3\end{array}$ & $\begin{array}{r}A(I, J, 1) \\
1.754 E+03 \\
1.174 E+03 \\
-1.316 E+03 \\
-5.499 E+02 \\
-1.128 E+02 \\
-3.580 E+01 \\
-6.400 E+01 \\
7.096 E+00 \\
-1.082 E+01 \\
3.844 E+00 \\
1.799 E+00\end{array}$ & $\begin{array}{l}A(I, J, 2) \\
0 . \\
1.370 E+02 \\
5.218 E+03 \\
7.881 E+03 \\
3.912 E+03 \\
1.578 E+03 \\
3.084 E+03 \\
1.671 E+13 \\
5.919 E+03 \\
4.077 E+02 \\
3.181 E+03\end{array}$ & $\begin{array}{l}A(I, J, 3) \\
-4 . \\
-4.815 E+D 0 \\
-1.960 E+D 3 \\
5.864 E+D 3 \\
3.230 E+D 4 \\
1.290 E+155 \\
1.751 E+165 \\
3.267 E+65 \\
2.475 E+D 6 \\
3.137 E+D 6 \\
1.898 E+06\end{array}$ & 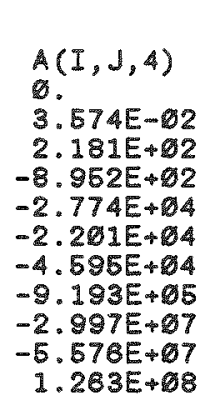 \\
\hline $\begin{array}{l}1 \\
2 \\
3 \\
4 \\
5 \\
6 \\
7 \\
8 \\
9 \\
6\end{array}$ & $\begin{array}{c}\text { EUROPIUM } \\
.91--.93105 \\
.0305--N 5 \\
\text { NE--ME } \\
M 5--M 3 \\
M 3--M 2 \\
M 2--M 1 \\
M 1--L 3 \\
L 3--L 2 \\
L 2--L 1 \\
L 1--K \\
K--100 . \\
100 .--500 . \\
500 .--I N F\end{array}$ & $\begin{array}{l}/ A=4 . \\
.01 \\
.0385 \\
.134 \\
1.131 \\
1.481 \\
1.814 \\
1.810 \\
6.977 \\
7.818 \\
8.852 \\
48.519 \\
10 \varnothing . \\
500 .\end{array}$ & $\begin{array}{l}148 E-01 \\
.0305 \\
.134 \\
1.131 \\
1.481 \\
1.614 \\
1.806 \\
6.977 \\
7.618 \\
8.052 \\
48.519 \\
100 . \\
\text { 506. } \\
\text { INF }\end{array}$ & $\begin{array}{c}N A \\
9.2-2 \\
5.1-1 \\
3.7-5 \\
4.7-7 \\
1.5-8 \\
3.5-3 \\
1.3-7 \\
4.1-8 \\
3.9-3 \\
2.6-3\end{array}$ & $\begin{array}{r}2.047 E+03 \\
4.278 E+03 \\
-4.678 E+02 \\
-6.507 E+02 \\
-4.589 E+02 \\
-4.913 E+02 \\
-2.288 E+02 \\
-2.465 E+01 \\
-6.266 E+11 \\
-4.306 E+00 \\
-7.610 E+100 \\
3.783 E+00 \\
1.904 E+00\end{array}$ & $\begin{array}{l}\text {. } \\
-4.382 E+02 \\
4.034 E+03 \\
7.938 E+\emptyset 3 \\
1.019 E+04 \\
8.746 E+03 \\
5.934 E+03 \\
1.115 E+03 \\
3.143 E+03 \\
2.028 E+03 \\
5.488 E+03 \\
7.655 E+\emptyset 2 \\
3.460 E+03\end{array}$ & 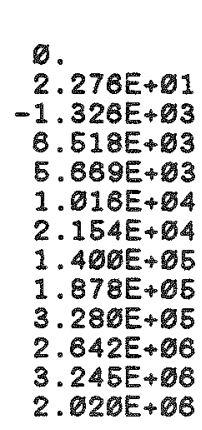 & 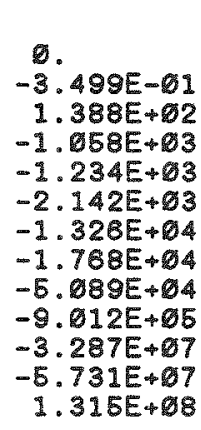 \\
\hline $\begin{array}{r}1 \\
2 \\
3 \\
4 \\
5 \\
6 \\
7 \\
8 \\
9 \\
10\end{array}$ & $\begin{array}{c}\text { GADOLINIUM } \\
.11=-.9305 \\
.0305--N 5 \\
\text { NE--M5 } \\
M 5--M 3 \\
M 3--M 2 \\
M 2--M 1 \\
M 1--L 3 \\
L 3--L 2 \\
L 2--L 1 \\
L 1--K \\
K--500 . \\
500 .--I N F\end{array}$ & $\begin{array}{l}1 A=4 . \\
.01 \\
.0365 \\
.140 \\
1.185 \\
1.544 \\
1.888 \\
1.881 \\
7.243 \\
7.93 \\
8.375 \\
56.239 \\
560 .\end{array}$ & $\begin{array}{l}\text { D7פE- } 11 \\
.5365 \\
.140 \\
1.185 \\
1.544 \\
1.688 \\
1.881 \\
7.243 \\
7.93 \\
8.375 \\
58.239 \\
500 . \\
\text { INF }\end{array}$ & $\begin{array}{c}\text { NA } \\
7.2-2 \\
1.2+1 \\
3.5-5 \\
3.5-7 \\
1.4-6 \\
2.9-3 \\
3.4-7 \\
2.2-8 \\
3.3-3\end{array}$ & $\begin{array}{r}1.620 E+03 \\
1.627 E+03 \\
-2.404 E+03 \\
-5.394 E+02 \\
-4.486 E+02 \\
-4.679 E+02 \\
-3.017 E+02 \\
-5.768 E+01 \\
-3.358 E+01 \\
-8.668 E+00 \\
3.209 E+00 \\
1.968 E+00\end{array}$ & $\begin{array}{l}6 . \\
8.32 \\
7.22 \\
7.8 \\
1.02 \\
9.42 \\
6.83 \\
2.8 \\
1.78 \\
2.37 \\
1.57 \\
3.5\end{array}$ & 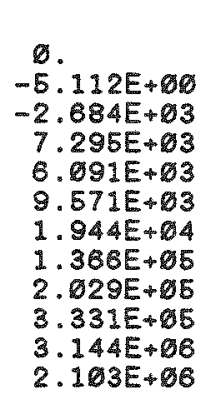 & $\begin{array}{r}\text { } . \\
7.828 E-82 \\
2.976 E+82 \\
-1.218 E+\varnothing 3 \\
-1.380 E+\varnothing 3 \\
-2.201 E+83 \\
-1.104 E+84 \\
-4.131 E+84 \\
-3.312 E+\varnothing 4 \\
-9.285 E+\varnothing 5 \\
-4.804 E+\varnothing 7 \\
1.345 E+\varnothing 8\end{array}$ \\
\hline $\begin{array}{r}1 \\
2 \\
3 \\
4 \\
5 \\
6 \\
7 \\
8 \\
9 \\
10 \\
11 \\
12\end{array}$ & $\begin{array}{c}\text { TERBIUM } \\
.01--.6305 \\
.6305--N E \\
N 5--M E \\
M 5--M 3 \\
M 3--M 2 \\
M 2--M 1 \\
M 1--L 3 \\
L 3--L 2 \\
L 2--L 1 \\
L 1--K \\
K--500 . \\
500 .--I N F\end{array}$ & $\begin{array}{l}=4.09 \\
.01 \\
.0365 \\
.147 \\
1.24 \\
1.61 \\
1.765 \\
1.963 \\
7.514 \\
8.252 \\
8.708 \\
51.996 \\
568 .\end{array}$ & $\begin{array}{l}9 \mathrm{E}-11 \\
.8365 \\
.147 \\
1.24 \\
1.61 \\
1.785 \\
1.983 \\
7.514 \\
8.252 \\
8.708 \\
51.996 \\
509 . \\
\text { INF }\end{array}$ & $\begin{array}{c}N A \\
9 \cdot 9-2 \\
4 \cdot 5-1 \\
3 \cdot 3-5 \\
7 \cdot 5-7 \\
1.4-6 \\
1.4-3 \\
8 \cdot 7-7 \\
2 \cdot 8-8 \\
5.3-3\end{array}$ & $\begin{array}{l}1 . \\
3 . \\
-1 . \\
-5 . \\
-4 . \\
-4 . \\
-1 . \\
-1 . \\
-4 . \\
-4 . \\
3 . \\
2 .\end{array}$ & 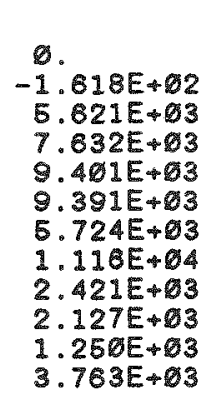 & $\begin{array}{r}8 . \\
9.9 \\
-1.9 \\
8.8 \\
9.2 \\
1.1 \\
2.8 \\
8.7 \\
2.1 \\
3.6 \\
3.4 \\
2.2\end{array}$ & $\begin{array}{r}1 . \\
-1.862 E-01 \\
2.675 E+02 \\
-1.449 E+63 \\
-2.000 E+03 \\
-2.613 E+03 \\
-1.782 E+04 \\
-7.647 E+64 \\
-4.859 E+04 \\
-1.159 E+08 \\
-5.837 E+07 \\
1.155 E+188\end{array}$ \\
\hline $\begin{array}{r}1 \\
2 \\
3 \\
4 \\
5 \\
8 \\
7 \\
8 \\
9 \\
10 \\
11\end{array}$ & $\begin{array}{c}\text { DYSPROSIUM } \\
\text { D1--.D305 } \\
.0305--N 5 \\
N 5--M 5 \\
M 5--M 3 \\
M 3--M 2 \\
M 2--M 1 \\
M 1--L 3 \\
L 3--L 2 \\
L 2--L 1 \\
L 1--K \\
K--5 \varnothing 0 . \\
\text { 500.--INF }\end{array}$ & $\begin{array}{l}2 / A= \\
.01 \\
.0365 \\
1.154 \\
1.295 \\
1.876 \\
1.842 \\
2.848 \\
7.79 \\
8.58 \\
9.046 \\
53.788 \\
500 .\end{array}$ & $\begin{array}{l}4.062 E-0 \\
.0305 \\
.154 \\
1.295 \\
1.678 \\
1.842 \\
2.048 \\
7.79 \\
8.58 \\
9.646 \\
53.788 \\
\text { EøD. } \\
\text { INF }\end{array}$ & $\begin{array}{l}N A \\
1 \cdot 5-1 \\
2 \cdot 8-1 \\
3 \cdot 1-5 \\
7 \cdot 5-7 \\
1 \cdot 3-8 \\
2 \cdot 0-3 \\
5 \cdot D-7 \\
4 \cdot 2-8 \\
4 \cdot 3-3 \\
4 \cdot 8-3 \\
2 \cdot 4-2\end{array}$ & $\begin{array}{l}3.28 \\
3.18 \\
-1.18 \\
-5.2 \\
-4.68 \\
-4.68 \\
-1.08 \\
-6.32 \\
-7.28 \\
-1.88 \\
4.12 \\
2.18\end{array}$ & $\begin{array}{r}9 . \\
-4.242 E+01 \\
5.234 E+03 \\
7.751 E+03 \\
1.650 E+04 \\
9.448 E+03 \\
4.675 E+03 \\
3.407 E+03 \\
4.178 E+03 \\
3.374 E+03 \\
1.344 E+63 \\
3.928 E+03\end{array}$ & $\begin{array}{l}0 . \\
5.269 E+00 \\
-1.652 E+03 \\
9.341 E+03 \\
8.066 E+03 \\
1.216 E+04 \\
3.253 E+04 \\
1.499 E+05 \\
2.136 E+05 \\
3.526 E+05 \\
3.520 E+06 \\
2.396 E+06\end{array}$ & $\begin{array}{l}\text { 6. } \\
-1.178 E-01 \\
1.694 E+12 \\
-1.824 E+03 \\
-1.930 E+03 \\
-2.942 E+03 \\
-2.519 E+104 \\
-5.526 E+64 \\
-8.026 E+104 \\
-9.982 E+15 \\
-5.867 E+07 \\
1.987 E+08\end{array}$ \\
\hline
\end{tabular}




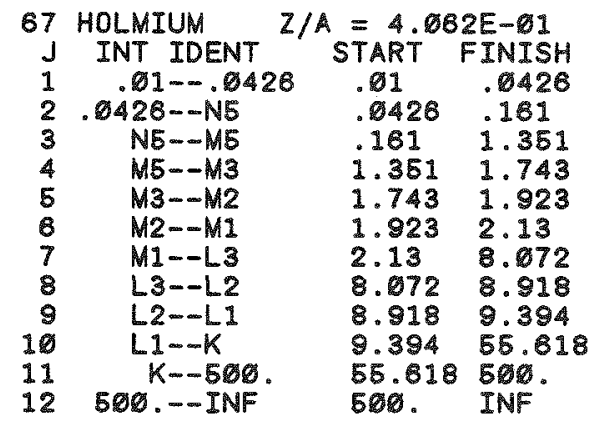

RMS
NA
$1 \cdot 4-1$
$3 \cdot 1-1$
$2 \cdot 8-5$
$1 \cdot 0-6$
$1 \cdot 2-6$
$4 \cdot 0-3$
$4 \cdot 5-7$
$2 \cdot 5-8$
$5 \cdot 4-3$
$2 \cdot 2-2$
$2 \cdot 4-2$

NA

2. $6-2$

1. 6-2

$2.8-5$

$1.2-8$

$1.1-6$

$3.6-3$

$5.5-7$

$2.9-8$

1. $1-2$

8. $0-3$

$2 \cdot 3-2$

NA

8.6-2

$5.4-2$

$2 \cdot 4-5$

8. $0-2$

$6.3-7$

2. $9-8$

$9.4-3$

$8.4-3$

2. 4-2

$$
\begin{aligned}
& A(I, J, 1) \quad A(I, J, 2) \\
& \text { 1.847E+D3 } \\
& 2.949 E+63-5.473 E+01 \\
& -1.961 E+13
\end{aligned}
$$

$2.419 E+3$.

$3.178 E+5-8.946 E+01$ $-1.598 E+03$

$-5.148 E+02$

$-4.781 E+82$

$-4.476 E+22$

$-2.279 E+02$

$-5.162 E+11$

$-5.360 E+51$

$6.458 E+09$

$4.025 E+60$

$2.388 E+06$

$6.371 E+83$

$8.464 E+03$

$8.646 \mathrm{E}+13$

$9.498 \mathrm{E}+63$

$6.501 E+163$

2. $947 E+13$

3. $254 E+03$

1. $211 E+\infty 3$

2. $.31 E+93$

$4.497 E+103$

$1.729 E \rightarrow 03$

$5.004 E+03-3.383 E+102$

$-1.721 E+03$

$-5.074 E+82$

$4.504 E+82$

$-5.189 E+61$

$-5.311 E+01$

$-3.887 E+00$

$4.124 E+60$

$2.512 E+\varnothing 6$

$6.821 E+03$

$8.420 E+103$

$-4.717 E+103$

$3.077 E+D 3$

$3.348 E+13$

$2.132 E+13$

$2.299 E+\square 3$

$4.774 E+13$

$2.440 E+83$

$500 .--$ INF
10.48961 .33

61.332500.

506. INF
6. $2-2$

$4 \cdot 5-2$

2. $4-5$

$7 \cdot 8-2$

$7.5-7$

$3.7-8$

3. $8-3$

$5.1-3$

$2.3-2$

$5.923 E+03-5.235 E+22$

$-1.482 E+13$

$-4.649 E+62$

$3.677 E+02$

$-5.328 E+01$

$-7.311 E+61$

$-2.434 E+01$

$4.575 E+00$

$2.888 \mathrm{E}+00$ 8. $524 E+83$ $-2.145 E+83$ $3.291 E+53$ $4.929 E+83$ $4.289 E+83$ $2.312 E+63$ $4.980 E+63$

$2.123 E+103$

5. 2-2 $4 \cdot 1-2$ $2.1-5$ $7 \cdot 2-2$ $8.5-7$ $2.6-8$ 1. -2 4. 2-3 2. 4-2

RMS

$7.5-2$ $3 .-2$ $4 \cdot 2-2$ $2 \cdot 6-5$ $7 \cdot 0-2$ 1. $1-6$ $3 \cdot 3-8$ $3 \cdot 1-3$ $3 \cdot 5-3$ $2 \cdot 5-2$

$8.368 E+03-1.105 E+103$

$-1.538 E+037.063 E+13$

$-4.936 E+029.095 E+63$

$2.304 E+12-1.379 E+183$

$-5.213 E+01 \quad 3.333 E+03$

$-8.333 E+01 \quad 3.627 E+63$

$3.726 E+61.486 E+63$

$5.286 E+60 \quad 2.137 E+63$

$2.736 E+5.269 E+3$

$A(I, J, 1) \quad A(I, J, 2)$

$5.463 E+3-2.212 E+82$

$9.488 E+103-1.416 E+83$

$-1.796 E+037.963 E+83$

$-4.835 E+229.549 E+b 3$

$3.480 E+22-3.031 E+83$

$-6.090 E+11.112 E+23$

$-7.386 E+15.387 E+83$

$-1.873 E+1 \quad 4.102 E+03$

$4.753 E+02.673 E+03$

$$
A(I, 1,3)
$$

D.

$A(I, J, 4)$

o.

$-1.313 E+00 \quad 5.447 E-02$

$-2.353 E+63 \quad 2.538 E+02$

. $852 E+103-1.807 E+163$

$1.119 E+04-2.580 E+63$

$1.378 E+04-3.393 E+03$

$3.428 E+4-2.787 E+24$

$1.628 E+65-5.658 E+124$

$2.349 E+05-5.948 E+04$

$4.072 E+85-1.335 E+06$

$4.000 E+08-7.974 E+07$

$2.387 E+06 \quad 1.450 E+08$

$U$
$Y$
$Y$
$Y$
$N$
$N$
$N$
$N$
$N$
$N$
$N$

0.

.

$1.463 E+04-3.195 E+83$

$1.489 E+2-3.799 E+63$

$3.083 E+04-2.363 E+24$

$1.716 E+05-5.828 E+04$

$2.449 E+05-7.821 E+24$

$4.583 E+05-1.884 E+D 6$

$3.795 E+06-6.651 E+07$

$2.558 E+06 \quad 1.493 E+68$

0.

$.358 E+01-1.932 E-51$

$191 E+03 \quad 2.162 E+02$

$1.161 E+04-2.277 E+103$

$9.421 E+04-1.104 E+65$

$1.888 E+05-6.499 E+04$

$2.598 E+05-8.657 E+04$

$4.637 E+05-1.723 E+06$

$3.958 E+06-7.643 E+07$

$2.690 E+06 \quad 1.454 E+08$

0.

0.

$2.450 E+01-3.644 E-91$ $-1.996 E+83 \quad 1.849 E+12$

$8.110 E+83-1.884 E+23$

$8.374 E+04-9.504 E+04$

$1.869 E+85-7.332 E+04$

$2.802 E+05-1.239 E+05$

$4.243 E+D 5-1.379 E+06$

$4.086 E+06-7.562 E+07$

$2.895 E+06 \quad 9.923 E+07$

6.

0

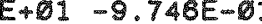

$2.291 E+03 \quad 2.250 E+02$

$1.255 E+04-2.717 E+03$

$8.594 E+04-1.012 E+05$

$1.974 E+05-7.977 E+04$

$2.858 E+05-1.071 E+05$

$5.352 E+65-2.222 E+66$

$4.329 E+86-8.327 E+07$

$3.153 E+66 \quad 5.239 E+67$

$A(I, J, 3) \quad A(I, J, 4)$

3.566E+100 - $1.819 E-02$

$8.676 E+\emptyset 1-1.382 E+\varnothing 6$

$-2.748 E+63 \quad 2.932 E+92$

$1.284 E+04-2.957 E+03$

$9.762 E+64-1.203 E+85$

$2.015 E+05-9.934 E+54$

$2.859 E+65-1.536 E+65$

$4.898 E+65-1.572 E+06$

$4.462 E+06-8.960 E+\varnothing 7$

$3.013 E+86 \quad 1.535 E+08$ 
73 TANTALUM

$\begin{array}{rc}J & \text { INT IDENT } \\ 1 & .01--.0233 \\ 2 & .0233-. .0428 \\ 3 & .0428--N 5 \\ 4 & N 5--M 5 \\ 5 & M 5--M 3 \\ 8 & M 3--L 3 \\ 7 & \text { L3--L2 } \\ 8 & L 2--L 1 \\ 9 & \text { L1--K } \\ 10 & K--566 . \\ 11 & 560 .--I N F\end{array}$

$Z / A=4.034 E-01$ START FINISH O1 .0233 .0428 .0428 .229 2291.735 $1.735 \quad 2.194$ 2.1949 .880 9.88011 .138 $11.136 \quad 11.68$ $11.88 \quad 87.418$ 87.416580 56ீ. INF
RMS

7. 3-2

3.3-2

1. 2-1

4.2-2

$1.8-5$

$5.9-2$

$1.2-8$

2.2-8

$9.7-3$

$3 \cdot 2-3$

$2.2-8$

1. 2-1

2.1-2

2. $0-1$

3. 8-2

1. 6-5

3. 8-2

1. 5-6

$4 \cdot 9-8$

2. $8-3$

2. $8-3$

2. 5-2

1.1-1

4. 2-1

1. 1-1

1. 4-5

4. 7-2

1. 8-6

$4 \cdot 8-8$

$5 \cdot 2-3$

3. $5-3$

2. $4-2$

$500 .--I N F$

$\begin{array}{ll}71.678 & 56 \emptyset \\ 50 \emptyset . & \text { INF }\end{array}$

$Z / A=3.996 E-01$

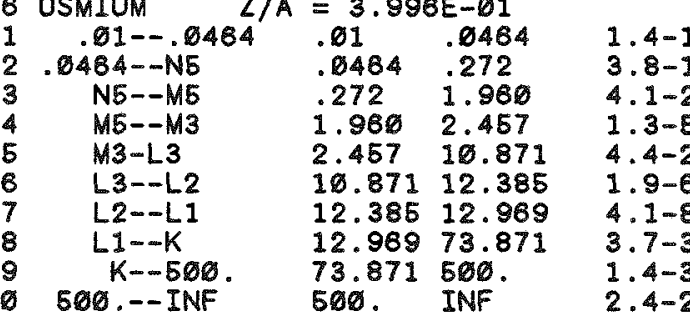

77 IRIDIUM

$$
\begin{aligned}
& .61--.048 \\
& N 8--N 5 \\
& M 5--M 5 \\
& M 3--L 3 \\
& L 3--L 2 \\
& L 2--L 1 \\
& L 1--K \\
& K--506 .
\end{aligned}
$$

500.--INF
$Z / A=4.006 E-01$

$.01 \quad .048$

$.048 \quad .295$

.2952 .040

$2.040 \quad 2.551$

2.55111 .216

$11.215 \quad 12.824$

$12.824 \quad 13.419$

13.41976 .111

76.111566.

500. INF

$Z / A=3.998 E-81$

$.01 \quad .0226$

.0226 .0735

$.0735 \quad .313$

$.313 \quad 2.122$

$2.122 \quad 2.845$

$2.645 \quad 11.564$

$11.564 \quad 13.273$

$13.273 \quad 13.88$

13.8878 .395

78.395500 .

5DE. INF
$A(I, j, 1)$

$3.537 E+04-2.591 E+03-6.585 E+\varnothing 1-5.643 E-81$

$9.080 E+03-1.376 E+03 \quad 8.354 E+\emptyset 1-1.487 E+00$

$-1.944 E+03 \quad 8.496 E+03-3.040 E+03 \quad 3.330 E+02$

$-4.771 E+02 \quad 1.053 E+04 \quad 1.338 E+04-3.254 E+103$

$1.047 E+02$

$-5.521 E+81$

$-4.987 E+01$

$-2.176 E+80$

$5.194 E+80$

$2.973 E+B$

$6.524 E+62$

$3.828 E+103$

$3.641 E+13$

$2.172 E+03$

$2.794 E+103$

$5.785 E+103$

8.380E+ $1.1 .625 E+05$

$2.145 E+05-1.019 E+\emptyset 5$

$3.168 E+\varnothing 5-1.250 E+05$

$5.708 E+05-2.482 E+06$

$4.645 E+66-9.648 E+67$

$3.328 E+180 \quad 9.868 E+\$ 7$

$-2.084 E+103$

$-1.398 E+24$

$9.985 E+103$

$-1.825 E+163$

$-4.686 E+12$

$1.412 E+12$

$-6.206 E+101$

$-1.211 E+02$

$-2.165 E+01$

$5.276 E+06$

$1.834 E+02-2.686 E+00 \quad 1.131 E-02$

$2.451 E+03-1.057 E+62 \quad 1.381 E+80$

$-1.816 E+03 \quad 1.301 E+62-2.747 E+60$ $8.623 E+03-3.114 E+63 \quad 3.432 E+02$

$1.057 E+\emptyset 4 \quad 1.355 E+04-3.503 E+83$

$2.762 E+22$

$4.525 E+103$

1. $058 \mathrm{E}+04$

4.623E+83

$2.998 E+63$

$3.099 E+100$

6.112E+ 03

$1.355 E+84-3.503 E+\varnothing 3$
$8.940 E+84-1.129 E+\varnothing 5$

$2.194 E+\varnothing 5-1.223 E+\varnothing 5$

$2.701 E+05-2.566 E+05$

$5.109 \mathrm{E}+\varnothing 5-1.835 \mathrm{E}+\varnothing 6$

$4.828 E+86-1.030 E+08$

$3.348 E+16 \quad 1.310 E+\varnothing 8$ $1.235 E+04-2.571 E+03 \quad 1.879 E+02-4.072 E+\varnothing 0$ $-1.846 E+03 \quad 9.198 E+03-3.553 E+03 \quad 4.281 E+02$ $-4.438 E+02 \quad 1.148 E+04$ $1.961 E+102-3.585 E+62$

$-5.721 E+01 \quad 4.284 E+63$

$-1.192 E+102 \quad 1.681 E+\$ 4$

$-8.548 E+1003.122 E+83$

$4.901 E+90$

$3.621 E+\emptyset 3$

$3.238 \mathrm{E}+186$

B. $600 E+03$

$1.253 E+\varnothing 4-3.520 E+\varnothing 3$

$9.550 \mathrm{E}+04-1.180 \mathrm{E}+05$

$2.331 E+85-1.278 E+\varnothing 5$

$2.828 E+\varnothing 5-2.788 E+\varnothing 5$

$5.972 E+\varnothing 5-2.571 E+\varnothing 6$

$4.969 E+166-1.085 E+108$

3. $237 \mathrm{E}+\varnothing 6 \quad 2.204 \mathrm{E}+188$

$5.189 E+102$

$9.943 E+103$

$-1.915 E+83$

$-4.312 E+82$

$-7.620 E+81$

$-5.948 E+181$

$-1.116 E+02$

$-1.803 E+01$

$5.735 E+00$

$3.351 E+80$

$1.085 \mathrm{E}+52$

$-2.362 E+\varnothing \varnothing \quad 1.285 E-\varnothing 2$ 1.499E+02-3.533E+80

$9.625 E+\varnothing 3-3.819 E+\varnothing 3 \quad 4.641 E+02$

$1.174 E+D 4 \quad 1.298 E+\varnothing 4-3.813 E+\varnothing 3$

$3.809 E+103$

$4.643 E+83$

1. $035 E+54$

4. $548 E+03$

$3.318 \mathrm{E}+03$

$6.829 E+83$

$8.189 E+04-1.096 E+105$

$2.451 E+05-1.434 E+05$

$3.012 E+65-2.961 E+65$

$5.706 E+15-2.246 E+06$

$5.232 E+66-1.209 E+\varnothing 8$

$3.589 E+186 \quad 1.867 E+188$

1. 8-1

$4.8-1$

3.6-2

1. 3-5

5. $1-2$

1. $8-6$

4. 8-8

1. 4-2

2. 4-3

$2.5-2$

$-9.441 E+9$ $9.926 E+\varnothing 3$

1. $284 E+62$ -2.049E+ 03

$-2.391 E+03 \quad 1.087 E+04$

$-4.371 E+02 \quad 1.150 E+04$

$1.540 \mathrm{E}+02-7.160 \mathrm{E}+\emptyset 2$

$-5.153 E+01 \quad 4.097 E+103$

$-1.366 E+02 \quad 1.456 E+04$

$-1.995 E+81 \quad 4.966 E+03$

$6.213 E+60$

3. $458 \mathrm{E}+183$

$3.505 E+00$

$7.126 E+13$

6. 1-2

8. $4-2$

$7 \cdot 9-1$

$3.7-2$

1. 2-5

$6.7-2$

$2.7-6$

4.6-8

1. 3-2

$3.4-3$

2.3-2
$1.220 E+04-3.768 E+02$

$1.396 \mathrm{E}+04-1.184 \mathrm{E}+03$

$1.172 E+104-2.752 E+103$

$-2.373 E+03$

$-4.333 E+02$

$-7.755 E+01$

$-6.719 E+01$

$-1.399 E+102$

$1.076 \mathrm{E}+01$

$6.088 \mathrm{E}+00$

$3.848 E+16$
1. $129 E+104$

$1.147 \mathrm{E}+04$

$2.847 E+63$

$5.737 E+103$

$1.606 \mathrm{E}+04$

$9.617 E+02$

$4.001 E+03$

$7.453 E+23$
$-2.563 E+00 \quad 1.347 E-02$ $1.836 E+02-4.025 E+16$ $1.539 E+04-4.566 E+03$ $1.147 E+05-1.673 E+\emptyset 5$ $2.582 E+05-1.419 E+05$ $2.707 E+05-3.461 E+05$ $5.442 E+06-1.286 E+08$ $3.865 E+66 \quad 1.114 E+\varnothing 8$

$4.167 E+\emptyset 0-1.564 E-02$ $3.650 E+01-3.548 E-01$ $2.212 E+02-4.579 E+00$ $-4.739 E+03 \quad 6.166 E+02$ 1.716E+104-5.181E+ 63 $1.047 E+05-1.599 E+\varnothing 5$ $2.565 E+05-1.880 E+15$ $2.671 E+05-3.742 E+\varnothing 5$ $7.776 E+05-4.207 E+86$ $5.552 \mathrm{E}+06-1.306 \mathrm{E}+08$ $3.931 E+\emptyset 6 \quad 1.228 E+168$ $-4.456 E+13 \quad 5.612 E+02$ $6.084 E+15-2.648 E+106$

$Y$
$Y$
$Y$
$Y$
$N$
$Y$
$N$
$N$
$N$
$N$
$N$

$Y$
$Y$
$Y$
$Y$
$N$
$Y$
$N$
$N$
$N$
$N$
$N$

$Y$
$Y$
$Y$
$N$
$Y$
$N$
$N$
$N$
$N$
$N$

$Y$
$Y$
$Y$
$N$
$Y$
$N$
$N$
$N$
$N$
$N$




$\begin{array}{rccl}79 & \text { GOLD } & \text { Z/A } & =4.011 E-01 \\ J & \text { INT IDENT } & \text { START } & \text { FINISH } \\ 1 & .01--.07 & .01 & .07 \\ 2 & .07--N 5 & .07 & .334 \\ 3 & N 5--M 5 & .334 & 2.206 \\ 4 & M 5--M 3 & 2.206 & 2.743 \\ 5 & M 3--L 3 & 2.743 & 11.919 \\ 6 & L 3--L 2 & 11.919 & 13.734 \\ 7 & L 2--L 1 & 13.734 & 14.353 \\ 8 & L 1--K & 14.353 & 80.725 \\ 9 & K--500 . & 80.725 & 506 . \\ 10 & 500 .-I N F & 500 . & \text { INF }\end{array}$

RMS
$1 \cdot 2-1$
$5 \cdot 7-1$
$4 \cdot 7-2$
$1 \cdot 1-5$
$3 \cdot 7-2$
$3 \cdot 6-8$
$3 \cdot 8-8$
$4 \cdot 4-3$
$9 \cdot 6-4$
$2 \cdot 3-2$

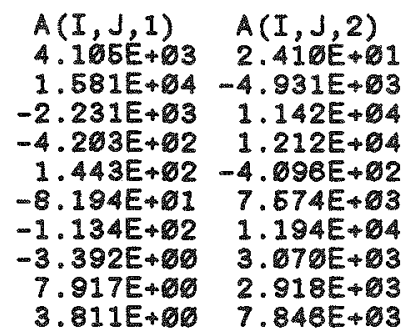

$A(I, j, 3)$

$A(I, J, 4)$

. $1.252 E-02$

$5.197 E+82-1.622 E+01$

$-4.781 E+63 \quad 6.012 E+22$

$1.692 E+04-5.448 E+103$

$1.249 E+65-1.944 E+65$

$2.508 E+95-2.318 E+65$

$3.355 E+05-3.921 E+65$

$7.247 E+05-3.499 E+06$

$8.095 E+06-1.598 E+28$

$4.248 E+06 \quad 4.571 E+07$

NA

$2.3+1$

$4 \cdot 3-2$

$9 \cdot 5-6$

3.9-2

3.9-6

$3.4-8$

$9.0-3$

$3.3-3$

$2.4-2$

NA

4.

9.7-1

4.1-2

9. $9-6$

3. $8-2$

$3.9-6$

$3 \cdot 9-8$

$5.4-3$

$2.8-3$

$2.4-2$

NA

5. 5-2

6. $7-1$

4. 2-2

$9.2-6$

4.2-2

$4.8-6$

$3.7-8$

$5.4-3$

4. $8-4$

2.2-2

88.004500

500.--INF

508 . INF

83 BISMUTH

$$
\begin{array}{r}
1 \\
2 \\
3 \\
4 \\
5 \\
6 \\
7 \\
8 \\
9 \\
18 \\
11 \\
12 \\
13
\end{array}
$$

$.01--05$

$05--04$

$04-.0379$

$Z / A=3.972 E-1$

.61 .024

.024 .028

.026 .0379

$.0379--.1136$

$1136--.362$

$.382--M 5$

ME - - M3

$M 3--L 3$

L3 $--L_{2}$

$L 2--L 1$

L1 - - K

$K=-500$.

$566 .-$ INF

.0379 .1136

$.1136 \quad .382$

.3622 .581

$2.581 \quad 3.177$

$3.177 \quad 13.42$

$13.42 \quad 15.714$

15.71416 .391

16.39190 .626

90.526500 .

500 .

INF

$\begin{array}{rc}84 & \text { POLONIUM } \\ 1 & .01--.0305 \\ 2 & .0305--.07 \\ 3 & .07--.2415 \\ 4 & .2415--M 5 \\ 5 & M 5--M 3 \\ 8 & M 3--L 3 \\ 7 & L 3--L 2 \\ 8 & L 2--L 1 \\ 9 & L 1--K \\ 10 & K--508 \\ 11 & 505 .--I N F\end{array}$

$Z / A=4.019 E-61$

.01 .0365

.0305 .07

$.67 \quad .2415$

.24152 .683

$2.883 \quad 3.302$

$3.302 \quad 13.814$

13.81418 .244

16.24416 .936

$16.936 \quad 93.105$

93.105500

500. INF

$6.5-2$

1. $0-2$

$1.8-2$

$5 \cdot 7-2$

$7 \cdot 2-1$

$1.2-1$

8.6-6

5. 1-2

$5.1-6$

$3.7-8$

$7.9-3$

$4 \cdot 5-3$

$2.3-2$
NA

$2.0-2$

$3.8-2$

$1.3-1$

$8.8-8$

$3.7-2$

$6.1-6$

$3 \cdot 5-8$

1. 20

2.2-3

2. $2-2$

$5.142 E+\square 3 \emptyset$.

$1.780 E+104-5.976 E+13$

$-2.317 E+63 \quad 1.206 E+04$

3.981E+52 $1.288 E+04$

$2.241 E+02-2.195 E+03$

$-7.533 E+11 \quad 7.084 E+03$

$-1.288 E+02$

$4.681 E-01$

$6.983 E+60$

$3.948 E+10$

$1.524 E+94$

$2.405 E+03$

$4.309 E+03$

$8.311 E+13$

8.

$6.765 E+82-2.176 E+1$

$-6.219 E+03 \quad 7.004 E+02$

$1.582 E+84-5.482 E+13$

$1.429 E+85-2.325 E+05$

$2.675 E+05-2.412 E+95$

$3.098 E+65-4.397 E+\varnothing 5$

$7.958 E+05-4.232 E+16$

$5.986 E+.6-1.518 E+08$

$4.256 E+\infty 6 \quad 8.942 E+07$

$1.823 E+03$

$1.488 E+04-4.878 E+03-7.721 E+01 \quad 9.111 E-01$

$.536 E+02-1.923 E+01$

$-4.087 E+02 \quad 1.156 E+04 \quad 2.169 E+04-7.188 E+63$

$1.851 E+02-9.046 E+102 \quad 1.464 E+65-2.314 E+65$

$-6.847 E+01 \quad 6.324 E+03 \quad 2.871 E+05-2.439 E+05$

$-1.405 E+02 \quad 2.137 E+04 \quad 2.340 E+05-4.290 E+05$

$-1.053 E+01 \quad 4.195 E+03 \quad 7.543 E+05-3.747 E+06$

$7.684 E+00 \quad 4.048 E+13 \quad 6.314 E+16-1.714 E+18$

$4.082 E+60 \quad 8.543 E+103$

$4.521 E+166 \quad 5.178 E+107$

$1.569 E+83 \quad 0$

$-9.851 E+163$

$1.869 E+64$

$-2.194 E+103$

$-4.016 E+52$

$6.749 E+61$

$-7 \cdot 125 E+\square 1$

$-1.383 E+12$

$-8.288 E+00$

$7.595 E+90$

$4.248 E+D B$

D.

$1.870 E+03-7.761 E+01 \quad 9.425 E-01$ $-5.855 E+\emptyset 37.149 E+02-2.672 E+01$ $1.239 E \rightarrow 04-5.418 E+03 \quad 6.794 E+62$ $1.173 E+042.316 E+04-7.955 E+03$ $6.789 E+62 \quad 1.461 E+05-2.437 E+05$ $7.128 E+63 \quad 2.931 E+05-2.779 E+95$ $1.992 E+D 4 \quad 2.721 E+65-4.887 E+65$ $4.058 E+03 \quad 7.950 E+05-4.115 E+06$ $4.692 E+83 \quad 6.429 E+06-1.747 E+108$ $8.883 E+93$

$4.754 E+06 \quad 3.448 E+27$

$-4.395 E+\theta$

$4.686 E+03-1.062 E+02$

$1.118 E+04$

$-1.578 E+04$

$2.124 E+104$

$-1.835 E+63$

$-3.943 E+62$

$2.482 E+02$

$-6.824 E+61$

$-1.337 E+02$

$-1.411 E+00$

$9.935 E+D 0$

$4.425 E+\varnothing 0$

$-3.159 E+02$

$2.207 E-61-1.865 E-03$ $-2.706 E+00 \quad 7.877 E-02$

$-9.250 E+00 \quad 2.918 E-\varnothing 1$ $-1.741 E+02 \quad 2.596 E+10$ $\begin{array}{rrr}8.884 E+03 & 1.240 E+03 & -5.312 E+01 \\ 1.188 E+04 & -5.123 E+03 & 6.229 E+02\end{array}$ $\begin{array}{rrr}1.188 E+04 & -5.123 E+63 & 6.229 E+02 \\ 1.129 E+04 & 2.871 E+04 & -9.164 E+63\end{array}$ $-2.657 E+83 \quad 1.837 E+165-2.887 E+85$ $7.005 E+\emptyset 3 \quad 3.089 E+05-2.951 E+05$ $1.999 E+04 \quad 2.963 E+05-5.342 E+05$ $3.094 E+03 \quad 8.901 E+05-5.203 E+86$ $3.289 E+03 \quad 6.983 E+06-2.020 E+D 8$ $9.396 E+83$

$5.868 E+06-5.286 E+07$

$1.109 E+63$

$1.939 E+64-1.380 E+03$

$9.152 E+\emptyset 3-3.829 E+\emptyset 3$

$-1.131 E+03 \quad 1.041 E+04$

$-3.899 E+62 \quad 1.134 E+64$

$4.918 E+02-1.053 E+04$

$-7.471 E+81 \quad 8.186 E+63$

$-1.335 E+62$

$7.789 E+60$

$8.525 E+16$

$4.633 E+90$
$2.199 E+64$

$1.767 E+83$

$5.162 E+83$

$8.888 E+\varnothing 3$ $\emptyset$.

$3.526 E+01-3.106 E-11$ $5.535 E+02-2.106 E+01$ $-4.126 E+83 \quad 4.235 E+82$ $2.963 E+04-1.038 E+8$ $2.383 E+165-4.647 E+05$ 3.143E+05 -3.397E+05 $2.786 E+D 5-5.569 E+85$ 1. $203 E+06-6.443 E+26$ $6.996 \mathrm{E}+66-2.069 \mathrm{E}+18$ $5.142 E+06 \quad 1.775 E+07$

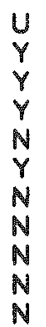

$Y$
$Y$
$Y$
$N$
$Y$
$N$
$N$
$N$
$N$
$N$ 


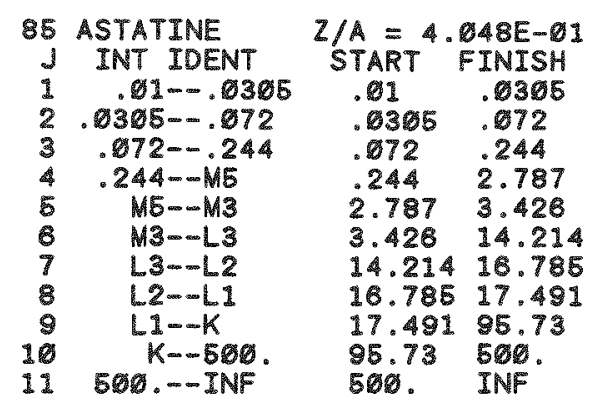

RMS
NA
$6.4-2$
$3.5+1$
$5.2-2$
$7.5-8$
$7.8-2$
$8.5-8$
$3.2-8$
$4.5-3$
$2.9-3$
$2.5-2$

NA

$\begin{array}{ll}.01 & .0486 \\ .0466 & .093 \\ .093 & .305 \\ .305 & 2.892 \\ 2.892 & 3.538 \\ 3.538 & 14.619 \\ 14.819 & 17.337 \\ 17.337 & 18.055 \\ 18.055 & 98.404 \\ 98.454 & 500 . \\ 506 . & \text { INF }\end{array}$

$Z / A=3.901 E-61$

.1 .049

$.0494 . .296$

$.196 \quad .525$

$.525 \quad 3.06$

$3.00 \quad 3.664$

3.66415 .63

$\begin{array}{ll}16.03 & 17.904\end{array}$

17.96418 .839

18.639181 .137

101.137500 .

500. INF

$500 .-$ INF
4. $8-2$

$3.4+0$

4. 7-2

$7.8-8$

5. $0-2$

7. 3-6

3. $1-8$

6. 5-3

3. $4-3$

2. $2-2$

NA

3.7-1

7. 8-1

2. $4-1$

6. 8-6

8. 6-2

$7.6-6$

3. $1-8$

$4.2-3$

4. 1-3

2. $4-2$

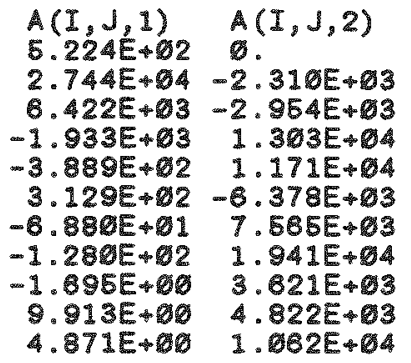

$A(I, 1,3)$

A

$6.658 E+61-658 E-01$ $4.933 E+62-2.019 E+\varnothing 1$ $-5.828 E+23 \quad 6.870 E+2$ $3.195 E+04-1.160 E+04$ $2.158 E+65-4.673 E+65$ 3. $411 E+65-3.547 E+65$ $3.508 E+65-8.558 E+25$ $9.663 E+15-5.746 E+96$ $7.370 E+26-2.163 E+08$ $5.352 E+66-2.792 E+67$

$1.284 E+53$

$-7.912 E+62$

$1.243 E+4$

$-1.804 E+03$

$-3.816 E+12$

$1.891 E+62$

$-6.756 E+61$

$-1.220 E+12$

$-5.899 E-11$

8.633E+6D

$4.841 E+80$ $-6.150 E+63$ 1. $279 E+04$ $1.088 E+04$ $-2.424 E+163$ $7.739 E+103$ $1.976 E+84$ $3.312 E+63$ 5. $888 E+13$ 1. $.57 E+104$

$1.287 E+53$ 3. $1.217 E+04-1.865 E+63-1.031 E+03 \quad 1.980 E+02$ $-2.201 E+03 \quad 1.461 E+04-7.082 E+03 \quad 9.132 E+02$ $-3.615 E+02 \quad 1.160 E+04$ $-5.789 E+02 \quad 1.680 E+164$ $-6.236 E+01 \quad 7.249 E+03$ $-1.213 E+02 \quad 2.052 E+04$ $-8.453 E+60 \quad 4.660 E+83$ $1.104 E+1014.630 E+03$ $5.063 E+00$

1. $115 E+1$

$3.541 E+04-1.383 E+04$ $5.526 E+04-7.044 E+64$ $3.612 E+05-3.893 E+65$ $3.455 E+15-7.195 E+105$ $9.757 E+.5-6.010 E+106$ $7.768 E+86-2.445 E+68$ $5.668 E+\emptyset 6-1.082 E+\emptyset 8$
1.415E+3 .

2. $6-2$

$5.3-2$

4.6-2

6. 2-6

4. 4-2

$8.4-8$

$3.8-8$

$3.8-3$

6. $3-3$

2. 2-2

$-3.003 E+04$

$2.941 E+14$

$-3.547 E+02$

$2.933 E+22$

$-8.085 E+61$

$-1.192 E+12$

$-1.024 E+01$

$1.241 E+21$

$5.221 E+80$
509. - - INF

103.922508

$875 E+83-8$.

$-1.852 E+13 \quad 1.361 E+04$

$1.164 E+24$ $-6.829 E+63$

$287 E+23$

$2.119 E+84$

$5.310 E+03$

3. $727 E+83$

1. $188 E+64$

6.

. $.798 E+02 \quad 1.571 E+\varnothing 1$

$3.591 E+03-2.242 E+02$

$-6.123 E+03 \quad 6.294 E+102$

$3.753 E+04-1.492 E+04$

$2.528 E+05-5.565 E+85$

$3.762 E+\$ 5-4.181 E+05$

$3.506 E+65-7.644 E+65$

$9.758 E+85-5.773 E+66$

$8.367 E+86-2.833 E+\emptyset 8$

$5.739 E+68-9.046 E+07$

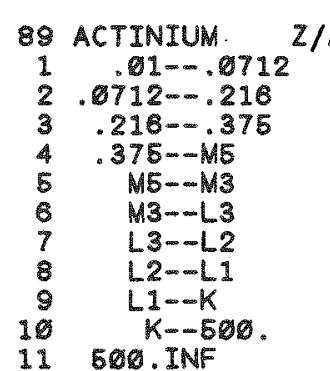

$/ A=3.920 E-91$

$\begin{array}{ll}.01 & .0712 \\ .0712 & .216 \\ .218 & .375 \\ .375 & 3.219 \\ 3.219 & 3.909 \\ 3.909 & 15.870 \\ 15.870 & 19.883 \\ 19.883 & 19.845 \\ 19.845 & 106.759 \\ 196.759500 . \\ 500 . & \text { INF }\end{array}$

NA

3. -2

1. $0+6$

4 . 3-2

5.8-6

4. $7-2$

$9.8-6$

$2.8-6$

6. $1-3$

3. $2-3$

2. $4-2$

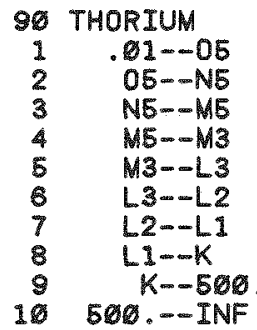

$Z / A=3.879 E-\varnothing 1$

$\begin{array}{lll}.01 & .0879 & N A \\ .8879 & .876 & 1.9+0 \\ .676 & 3.332 & 5.5-2 \\ 3.332 & 4.646 & 5.4-6 \\ 4.046 & 16.30 & 4.0-2 \\ 16.30 & 19.893 & 1.1-6 \\ 19.693 & 20.466 & 2.6-8 \\ 20.466 & 109.651 & 4.1-3 \\ 189.651 & 500 . & 3.8-3 \\ 509 . & \text { INF } & 2.4-2\end{array}$

$1.258 E+33$

$-2.747 E+03 \quad 3.975 E+22$

$1.958 E+04-9.878 E+03$

$-2.098 E+03 \quad 1.508 E+04$

$-3.474 E+62 \quad 1.153 E+84$

$5.789 E+2-1.597 E+04$

$-6.339 E+81 \quad 7.915 E+03$

$-1.182 E+82 \quad 2.194 E+84$

$8.142 E+60 \quad 2.287 E+83$

1. $083 \mathrm{E}+01 \quad 6.690 \mathrm{E}+03$

$5.479 E+100$

$1.217 E+04$

$1.134 E+63$

$1.398 E+84-6.113 E+83$

$-1.466 \mathrm{E}+03 \quad 1.349 \mathrm{E}+64$

$-3.308 E+102 \quad 1.094 E+104$

$2.265 E+02-3.798 E+63$

$-5.998 E+61 \quad 7.689 E+63$

$-1.152 E-62$

$-1.985 E+1$

$1.150 \mathrm{E}+81$

$5.620 \mathrm{E}+00$
$2.229 E+164$

$7.238 E+63$

$6.085 E+83$

1. $245 E+184$
6.

$1.941 E+62-1.439 E+81$

$1.388 E+83-1.778 E+81$

$-7.581 E+03 \quad 9.825 E+02$

$4.113 E+04-1.657 E+04$

$3.328 E+05-7.718 E+05$

$3.884 E+65-4.637 E+65$

$3.621 E+05-8.244 E+05$

$1.185 E+08-8.493 E+86$

$7.981 E+08-2.545 E+08$

$5.897 E+86-2.287 E+\varnothing 7$

2.

8 $.143 E+03 \quad 1.013 E+03$ $2.424 E+05-5.526 E+05$ $4.039 E+65-4.843 E+65$ $3.894 E+05-8.743 E+05$ $9.816 E+05-5.869 E+06$ $8.292 E+68-2.758 E+\$ 8$ 6. $338 E+106-1.549 E+68$

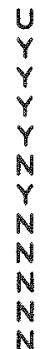

$Y$
$Y$
$Y$
$Y$
$N$
$Y$
$N$
$N$
$N$
$N$
$N$

$Y$
$Y$
$Y$
$Y$
$N$
$Y$
$N$
$N$
$N$
$N$
$N$ 


\begin{tabular}{|c|c|c|c|c|}
\hline $\begin{array}{l}4 \\
5 \\
8 \\
7 \\
8 \\
9\end{array}$ & $\begin{array}{c}\text { PROTACTINIUM } \\
\text { INT IDENT } \\
.01--05 \\
05--.371 \\
.371--M 5 \\
M 5--M 3 \\
M 3--L 3 \\
L 3--L 2 \\
L 2--L 1 \\
L 1--K \\
K--500 . \\
500 .--I N F\end{array}$ & $\begin{array}{l}Z / A= \\
\text { START } \\
.01 \\
.094 \\
.371 \\
3.442 \\
4.174 \\
18.733 \\
20.314 \\
21.105 \\
112.60 \\
500 .\end{array}$ & $\begin{array}{l}3.939 E-01 \\
\text { FINISH } \\
.094 \\
.371 \\
3.442 \\
4.174 \\
16.733 \\
20.314 \\
21.105 \\
112.601 \\
1580 . \\
\text { INF }\end{array}$ & $\begin{array}{l}\text { RMS } \\
\text { NA } \\
7.8-2 \\
5.2-1 \\
5.0-6 \\
1.0-1 \\
1.3-5 \\
2.5-8 \\
7.7-3 \\
3.0-3 \\
2.6-2\end{array}$ \\
\hline
\end{tabular}

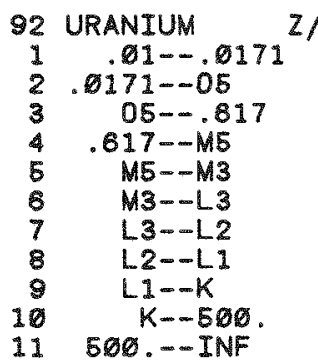

1. 4-1

5. 2-1

1. $8+1$

$6.0-2$

$4 \cdot 8-6$

$6.6-2$

1. $5-5$

$2 \cdot 3-8$

5. $8-3$

ป. $5-3$

$2 \cdot 3-2$

\begin{tabular}{|c|c|}
\hline 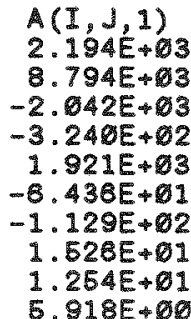 & $\begin{array}{r}A(I, J, 2) \\
0 . \\
-2.841 E+\emptyset 3 \\
1.581 E+\emptyset 4 \\
1.078 E+\emptyset 4 \\
-5.267 E+\emptyset 4 \\
8.617 E+\emptyset 3 \\
2.138 E+\emptyset 4 \\
1.358 E+\emptyset 3 \\
5.894 E+\emptyset 3 \\
1.306 E+\emptyset 4\end{array}$ \\
\hline
\end{tabular}

NA

6. $4-2$

$4.5-2$

$4 \cdot 5-6$

5. 9-2

1. $6-5$

2. $2-8$

$5 .-3$

$2.7-3$

2.6-2

$4.486 E+103-1.564 E+62$

$-2.175 E+03 \quad 4.327 E+62$

$1.528 E+04-7.108 E+03$

$-1.897 E+63 \quad 1.483 E+04$

$-1.100 E+02$

$-6.728 E+61$

$-1.033 E+2$

6. 281E-

1. $148 E+01$

$6.017 E+10$

$1.894 E+64$

$4.889 E+63$

$9.489 E+63$

$1.864 E+04$

$3.792 E+\$ 3$

7. $568 \mathrm{E}+13$

1. $327 E+4$

$2.522 E+13$

$1.668 E+04-8.686 E+83$

$-2.561 E+63$

$-3.232 \varepsilon+02$

$-4.467 E+51$

$-6.355 E+01$

$-1.193 E+82$

$5.579 E+100$

$1.272 E+101$

6. $330 E+10$

$1.868 E+04$

1. $200 E+64$

$2.778 E+63$

3. $093 \mathrm{E}+13$

2.501E+ +4

$3.489 E+03$

$7.422 E+63$

1. $392 E+14$

500. INF

$Z / A=3.852 E-21$

$$
94
$$

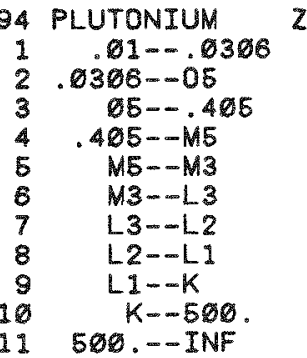

95 AMERICIUM

$\begin{array}{rc}1 & .21--05 \\ 2 & 05--.498 \\ 3 & 498--N 5 \\ 4 & N 5--M 5 \\ 5 & M 5--M 3 \\ 6 & M 3-L 3 \\ 7 & L 3--L 2 \\ 8 & L 2--L 1 \\ 9 & L 1--K \\ 10 & K=-500 \\ 11 & 500 .-I N F\end{array}$

NA

$1.8-2$

1. 3-2

$3 \cdot 5-1$

$4 \cdot 3-6$

4. $1-2$

$1.8-5$

2. $1-8$

$4 \cdot 5-3$

2. $5-3$

$2 \cdot 4-2$

NA

2.1-1

1.2-1

1. $5-2$

4. $0-6$

$5 \cdot 2-2$

1. $8-5$

1. $9-8$

2. $8-3$

3. $6-3$

2. $5-2$
$-3.148 E+02$

$3.568 E+63 \quad 0$.

$-2.939 E+13$

$1.270 E+04$

$-2.063 E+03$

$-3.181 E+82$

$3.925 E+92$

$-6.367 E+81$

$-1.065 E+02$

$-1.248 E+D 1$

1. $781 E+D 1$

6. $496 E+00$

8.00

0.

$+63-6.401 E+01$

$1.101 E+04$
$5.096 \mathrm{E}+04-2.302 \mathrm{E}+04$

$2.204 E+05-5.257 E+65$

$4.439 E+65-6.341 E+65$

$3.841 E+05-1.044 E+06$

$1.354 E+\emptyset 8-1.078 E+07$

$9.191 E+06-3.338 E+08$

$7.484 E+06-3.064 E+108$

6.

$-6.607 E+103 \quad 1.093 E+03-3.674 E+01$

$1.760 E+104-1.057 E+041.895 E+13$

$1.245 \mathrm{E}+104$

$-1.022 E+04$

$9.444 E+13$

$2.299 E+104$

$8.703 E+103$

$3.168 \mathrm{E}+13$

1. $422 E+4$

$9.698 E+020$.

$3.669 E+63-1.927 E+13$

$-8.896 E+04$

$-8.788 E+12$

$-3.188 E+92$

$3.232 E+02$

$-6.201 E+01$

$-9.199 E+81$

$-3.736 E+60$

1. $966 \mathrm{E}+1$

6. $763 E+\varnothing 0$

$1.824 E+\varnothing 5$

$1.063 E+\emptyset 4$

1. $325 E+04$

$-9.027 E+03$

$9.427 E+13$

$1.716 \mathrm{E}+04$

$5.425 E+13$

$2.217 E+03$

1. $479 E+64$

$5.098 E+\$ 4-2.427 E+104$

$3.515 E+05-9.309 E+05$

$4.539 E+55-6.824 E+65$

$4.461 E+05-1.181 E+06$

$1.250 E+06-9.411 E+06$

$1.075 E+07-4.693 E+08$

$8.179 E+D 8-5.34 D E+D 8$

6.

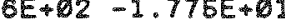

$19 E+052.150 E+104$

$5.553 E+02-1.758 E+03$

$5.127 E+04-2.586 E+04$

$3.584 E+05-9.931 E+95$

$4.778 E+05-7.281 E+105$

$6.101 E+85-1.350 E+06$

$1.376 E+06-1.109 E+07$

$1.154 E+67-5.298 E+88$

$8.847 E+96-6.116 E+98$

1.9-1

1. $0-1$

1.3-2

$3.7-6$

5. $8-2$

2. $1-5$

2. $0-8$

$4 \cdot 0-3$

4. $3-3$

2. $9-2$
$1.000 \mathrm{E}+030$.

$-7.979 E+14$

$-8.139 E+62$

$-3.144 E+82$

$-2.912 E+02$

$-5.879 E+D 1$

$-1.010 E+02$

$-1.147 E+01$

2. $200 E+11$
$7.017 E+80$
$1.716 \mathrm{E}+165$

1. $040 E+D 4$

1. $378 E+04$

1. $117 E+94$

$9.126 E+3$

$2.212 E+14$

$7.190 E+103$

1. $03 \mathrm{E}+3$
0

$2.954 E+02-1.411 E+01$ $-1.079 E+85 \quad 2.118 E+04$

$4.547 E+92-2.530 E+83$

$5.160 E+14-2.733 E+84$

$1.679 E+05-4.469 E+95$

$4.981 E+05-7.597 E+05$

$5.193 E+05-1.402 E+06$

$1.327 E+86-1.818 E+07$

$1.208 E+07-5.684 E+28$

$9.471 E+06-8.527 E+08$
$1.493 E+84$
$U$
$Y$
$Y$
$Y$
$Y$
$N$
$N$

$Y$
$Y$
$Y$
$Y$
$N$
$Y$
$N$
$N$
$N$
$N$
$N$

$Y$
$Y$
$Y$
$N$
$Y$
$N$
$N$
$N$
$N$
$N$

$500 .--$ INF 

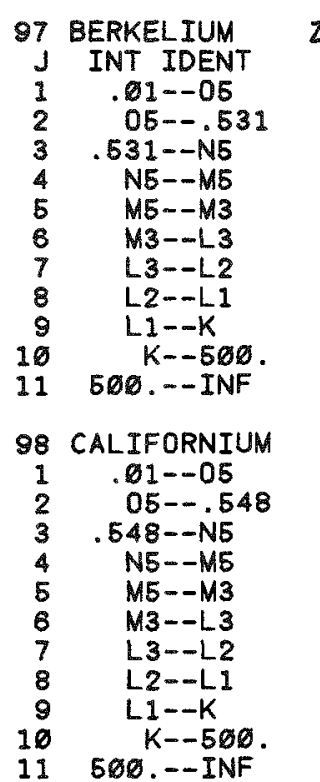

\section{EINSTEINIUM}

$$
\begin{gathered}
N 1--N 5 \\
N 5--M 5 \\
M 5--M 4 \\
M 4--M 3 \\
M 3--M 2 \\
M 2--M 1 \\
M 1--L 3 \\
L 3--L 2 \\
L 2--L 1 \\
L 1--K \\
K--5 \varnothing \emptyset . \\
500 .--I N F
\end{gathered}
$$

FERMIUM

$.91--N 5$
$N 5--M 5$
$M 5--M 4$
$M 4--M 3$
$M 3--M 2$
$M 2--M 1$
$M 1--L 3$
$L 3--L 2$
$L 2--L 1$
$L 1--K$
$K--5 \emptyset 0$
$500--I N F$

\section{$Z / A=3.927 E-01$ \\ START FINISH}

.1

.1

.531

.877

4.132

.531

4.132

$19.452 \quad 24.385$

$24.385 \quad 25.275$

$25.275 \quad 131.59$

131.59500 .

500. INF

$Z / A=3.904 E-91$

$.01 \quad .1 \quad$ NA

$.548 \quad .902$

$.902 \quad 4.254$

$4.254 \quad 5.109$

$5.109 \quad 19.929$

19.92925 .125

$25.125 \quad 26.03$

$26.03 \quad 135.005$

135.005500 .

500 .

INF

$Z / A=3.929 E-01$

$.01 \quad .927$

$927 \quad 4.378$

$4.378 \quad 4.630$

$4.630 \quad 5.259$

$5.259 \quad 6.574$

$6.574 \quad 6.977$

6.97720 .414

20.41426 .026

$26.02 \emptyset 26.906$

26.900139 .490

139.490500 .

500 .

INF

$Z / A=3.891 E-01$

$.61 \quad .952$

$.952 \quad 4.498$

$4.498 \quad 4.766$

$4.766 \quad 5.397$

$5.397 \quad 6.793$

$\begin{array}{lll}6.793 & 7.205\end{array}$

$\begin{array}{ll}7.205 & 20.907\end{array}$

20.90726 .810

$26.81027 .7 \varnothing \varnothing$

27.790143 .090

143.090500 .

500 .
RMS

NA

2. 1 1

5. 3-2

1. $7-2$

3. $4-6$

$5.9-2$

$2.4-5$

1.8-8

3. 6-3

4. 6-3

2. $8-2$

$1 \cdot 7-1$

$1.3-1$

1. 6-2

3. $0-6$

6. $0-2$

$2.6-5$

1.7-8

1. 2-2

6.6-3

3. 2-2

NA

1.4-2

1.3-7

$2.7-6$

$2.7-5$

1. 2-7

$4 \cdot 4-3$

2.9-5

1.8-8

$4.5-3$

9.2-3

2.6-2

\begin{abstract}
$A(I, J, 1)$
1.1ØळE+Ø3

$3.726 E+03$

$-1.348 E+65$

$-1.092 E+13$

$-3.168 \mathrm{E}+62$

$-8.536 E+101$

$-8.035 E+01$

$-1.018 E+02$

$-1.368 E+01$

$2.374 E+11$

$7.219 \mathrm{E}+00$
\end{abstract}

$A(I, J, 2)$

A.

$-1.998 E+03$

2. $867 E+95$

1. $396 E+D 4$

$5.205 E+103$

$9.752 E+83$

$2.538 E+04$

$7.916 \mathrm{E}+03$

$1.750 \mathrm{E}+02$

$1.592 \mathrm{E}+\$ 84$

$1.220 E+\emptyset 3$

$1.389 E+05-9.898 E+04-2.185 E+04$

$-9.356 E+02 \quad 1.188 E+104-4.863 E+102-2.379 E+03$

$-3.044 E+02 \quad 1.361 E+04 \quad 6.013 E+\varnothing 4-3.273 E+04$

$3.450 E+02-1.057 E+04 \quad 4.170 E+05-1.248 E+06$

$-5.797 E+\emptyset 1 \quad 9.571 E+83$

$-9.148 E+\varnothing 1 \quad 1.942 E+84$

$-5.416 E+01 \quad 1.706 E+04$

$2.771 E+01-2.903 E+83$

$5.352 E+05-8.755 E+65$

$6.490 E+65-1.673 E+06$

$8.638 E+65-1.792 E+86$

$1.419 E+\emptyset 7-7.456 E+\emptyset 8$

$1.052 E+\varnothing 7-1.089 E+\varnothing 9$

$1.330 \mathrm{E}+63$

$-8.936 E+62$

$-2.152 E+62$

$-2.914 E+\varnothing 2$

$-2.568 \mathrm{E}+82$

$-2.104 E+02$

$1.088 \mathrm{E}+02$

$-5.691 \mathrm{E}+81$

$-9.167 E+81$

$9.687 E-01$

$3.315 \mathrm{E}+01$

$7.728 \mathrm{E}+96$

ס.

$1.145 E+54$

$7.720 \mathrm{E}+03$

$1.288 E+84$

$1.341 \mathrm{E}+104$

$1.155 \mathrm{E}+\varnothing 4$

$1.536 E+63$

$9.668 \mathrm{E}+63$

$2.050 E+64$

$5.331 E+3$

$-7.298 E+03$

$1.675 E+104$

๑.

$1.594 E+83-3.692 E+63$

$5.372 E+84-2.276 E+64$

$6.828 E+04-3.620 E+64$

$8.885 E+84-5.387 E+04$

$1.107 E+05-7.279 E+104$

$2.458 E+05-6.065 E+05$

$5.558 \mathrm{E}+65-9.320 \mathrm{E}+05$

$6.529 E+05-1.784 E+06$

$1.646 E+86-1.518 E+97$

$1.587 E+07-8.866 E+\emptyset 8$

$1.99 \varnothing E+\varnothing 7-1.231 E+\varnothing 9$

$1.46 \varnothing E+\varnothing 3$

$1.6-2$

$1.4-7$

$2.2-6$

2.9-5

1. $1-7$

$2.1-3$

$3.2-5$

1. $5-8$

$5.2-3$

1.1-2

2.9-2
$-1.082 E+83$

$-1.923 E+02$

$-2.559 E+02$

$-2.466 E+102$

$-2.394 E+12$

$8.675 E+01$

$-5.793 E+81$

$-8.637 E+61$

$5.556 \mathrm{E}-81$

2. $968 E+\varnothing 1$

$8.135 \mathrm{E}+\varnothing 0$
$6.709 E+83$

$1.024 E+04$

$1.276 E+04$

$1.518 E+84$

$2.132 E+93$

$1.018 \mathrm{E}+04$

$1.896 E+04$

$5.848 E+183$

$-2.026 E+03$

1. $726 \mathrm{E}+104$
0.

$106 \mathrm{E}+02-2.846 \mathrm{E}+03$

$6.231 E+\emptyset 4-2.416 E+04$

$8.602 E+04-3.977 E+\$ 4$

$9.751 E+04-5.925 E+04$

$9.220 E+04-7.464 E+04$

$2.568 E+\varnothing 5-6.538 E+05$

$5.783 \mathrm{E}+65-1.018 \mathrm{E}+06$

$7.445 E+85-1.849 E+\varnothing 6$

$1.738 \mathrm{E}+68-1.662 \mathrm{E}+67$

$1.484 E+07-7.825 E+08$

$1.171 E+87-1.346 E+\varnothing 9$

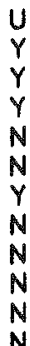

$$
\begin{aligned}
& Y \\
& Y \\
& Y \\
& N \\
& N \\
& Y \\
& N \\
& N \\
& N \\
& N \\
& N
\end{aligned}
$$

$N$
$N$
$N$
$N$
$N$
$N$
$N$
$N$
$N$
$N$
$N$
$N$




\section{APPENDIX B}

\section{Graphs}

\section{B.1 Discussion}

Although the cross-section representations described in this report for Klein-Nishina and photoelectric cross sections apply for all photon energies above $0.01 \mathrm{keV}$, we will show the plots in this Appendix only in the interval $0.01 \mathrm{keV} \leq \mathrm{E} \leq 1000 \mathrm{keV}$. The reason is that above $1000 \mathrm{keV}$, the photoelectric cross section becomes a smaller and smaller part of the total cross section. The total cross section is dominated by the scattering interaction, and the shape of this is shown in the Klein-Nishina plots given previously for photon energies up to $10^{6} \mathrm{keV}$ and tabulated to $10^{8} \mathrm{keV}$. Also at high energies $(>1.02 \mathrm{MeV})$ the pair production cross section increases in importance. We do not treat pair production in this document.

The photoelectric cross sections for each element were fitted to $\mathrm{Eq}(5)$ in several intervals. The boundaries for these intervals are given in the parameter table of Appendix A. The interval boundaries are often determined by absorption edges. The values of all the absorption edges that are used as interval boundaries are written on the plots and identified by name for your convenience.

The new fit to the photoelectric cross sections is given by the bottom solid curve of each plot. The old fit from our earlier compilation is shown by the dashed curve on each plot. The difference is most noticeable at low values of the photon energy.

The middle solid curve represents the energyabsorption cross section and is formed as the sum of the photoelectric cross section and the energytransferred part of the Klein-Nishina cross section. The top solid curve is the total cross section and consists of the sum of the photoelectric cross section and the total Klein-Nishina cross section.

We did not refit the photoelectric cross sections in the intervals for which the existing fit was adequate for this update. The parameter table in Appendix A indicates whether or not a refit was done by showing a $\mathrm{Y}$ or $\mathrm{N}$ in the last column.

For most of the elements, only a part of the source data is shown on the plots because the symbols would overlap too much to be legible. The thinning was done automatically by the computer and in some cases caused the fit to appear worse than it really is.
The difference between the old fit and the new one is clear on the plots at photon energies below 1 $\mathrm{keV}$, where much improved source data have become available during the last 15 years.

The high-energy limit behavior of the photoelectric cross section is governed by the coefficient of the $1 / \mathrm{E}$ term of $\mathrm{Eq}(5)$ for the highest energy interval. In our previous compilation, this coefficient was determined by theory for each element. In the current compilation it was necessary to use this coefficient in the fitting to adequately follow the cross-section source data at high photon energies. This resulted in a change of a few percent in these coefficients in some cases. This does not show on most of the plots because it usually occurs above the $1000 \mathrm{keV}$ upper limit of the graphs. However, this difference can be seen on some of the plots just below $\mathrm{E}=1000 \mathrm{keV}$; look at neon $(Z=10)$, for example.

The current compilation has more fitting intervals than the previous one. This was necessary to obtain adequate fitting accuracy. The root-meansquare fitting error for each interval is tabulated in the parameter table.

The fitting functions we are using (reciprocal powers of $\mathrm{E}$ ) in $\mathrm{Eq}(5)$ are more compatible for fitting monotonically decreasing functional dependence as occurs between absorption edges generally above 1 $\mathrm{keV}$. Below $1 \mathrm{keV}$ the photoelectric cross-section behavior often has a more complicated behavior, as can be observed by looking at the plots. By using shorter intervals we have still achieved some success in following the source data in this region. We used judgment as to when to stop improving the fits (at a cost of more fitting intervals) at these low energies. Source data are still scarce at very low photon energies, and there is some variation between sources.

Another point to make about the fitting at low photon energies is that to get an increasing function using a linear combination of decreasing functions, a differencing of large numbers often occurs. This may cause more roundoff error at low photon energies than at higher energies.

Another factor is that the cross sections become more dependent on the state of the absorbing material, where such things as molecular binding energies start to become comparable to the photon energies. 
On balance, we believe that we have represented the cross sections as well as the source data justify.

Another effect that should be mentioned is that the photoelectric cross section for a hydrogen atom would, strictly speaking, drop to zero below the $\mathrm{K}$ edge ( $\mathrm{E}=0.014 \mathrm{keV})$. However, we allowed it to drop only to well below the Klein-Nishina cross sections for free electrons at these energies for convenience in plotting and in computing.

For elements of atomic numbers $Z=96,97$, and 98 , we estimated the values of the $0_{\mathrm{v}}$ absorption edges. Values of the rest of the absorption edges were taken from a table in Reference 3, starting on page E-181.

\section{B.2 Symbols and Sources of Data}

The following table gives the sources of data used for fitting the parameters to the photoelectric cross sections. The symbols shown in this table are used throughout the 100 plots below. The superscripts in the Source of Data column refer to the corresponding references. Not all of these sources of data appear on every element. For example, the data from Smith et $a l$. appear only for aluminum $(\mathbb{Z}=13)$. Some of these sources are compilations.

\section{GRAPH SYMBOLS}

\begin{tabular}{|l|c|}
\hline \multicolumn{1}{|c|}{ Source of Data } & Symbol \\
\hline Henke, ot al. & \\
\hline LLNL6 & \\
\hline ENDF/B-V & \\
\hline DESY & \\
\hline Physik Daten $^{9,10}$ & \\
\hline Smith, et al. $^{11}$ & \\
\hline
\end{tabular}

\section{B.3 Graphs}

The graphs of the photon cross sections are contained on the next 100 pages. 


\section{HYDROGEN 1}

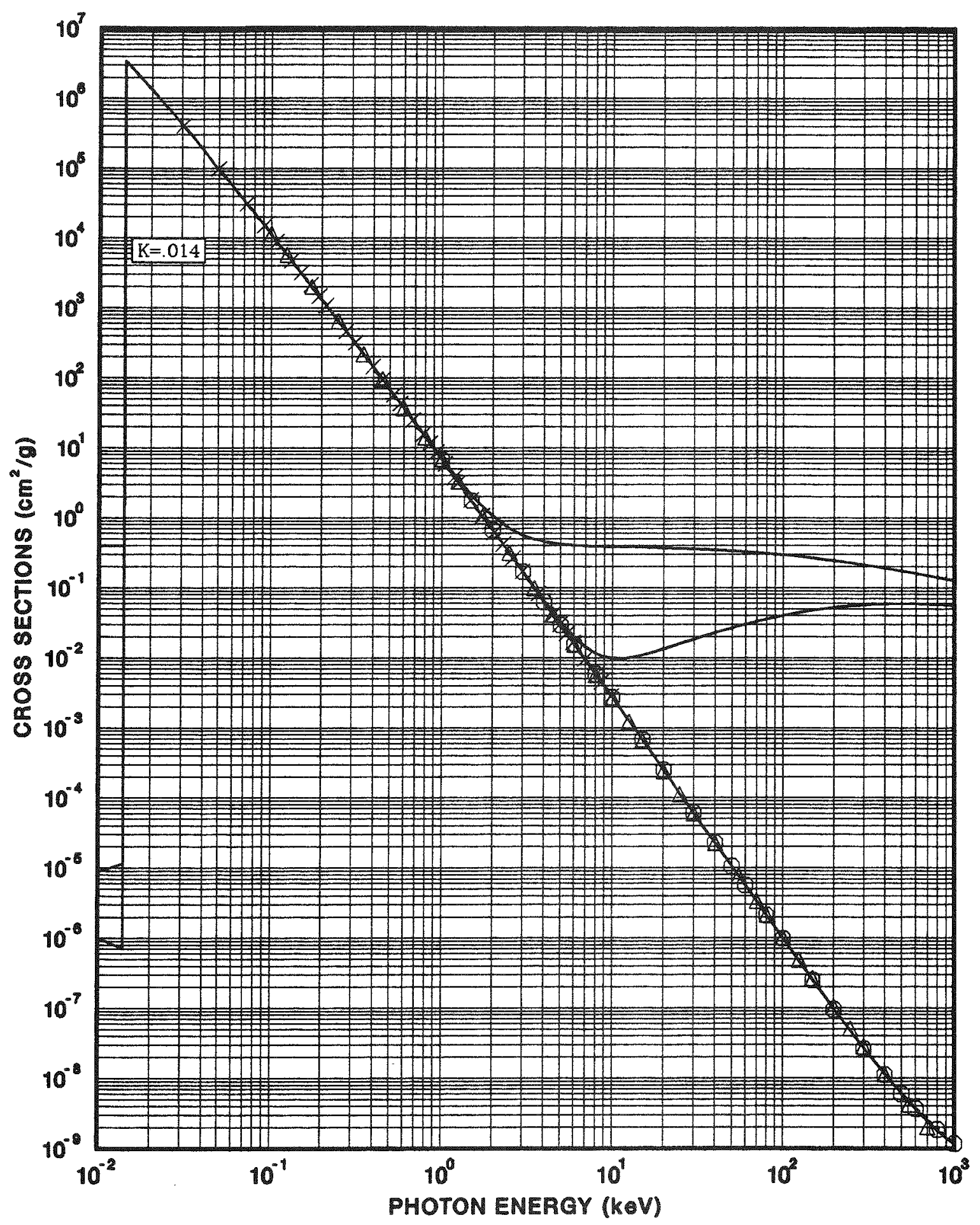

Barns/atom $=1.674 \times \mathrm{cm}^{2} / \mathrm{g}$ 
HELIUM 2

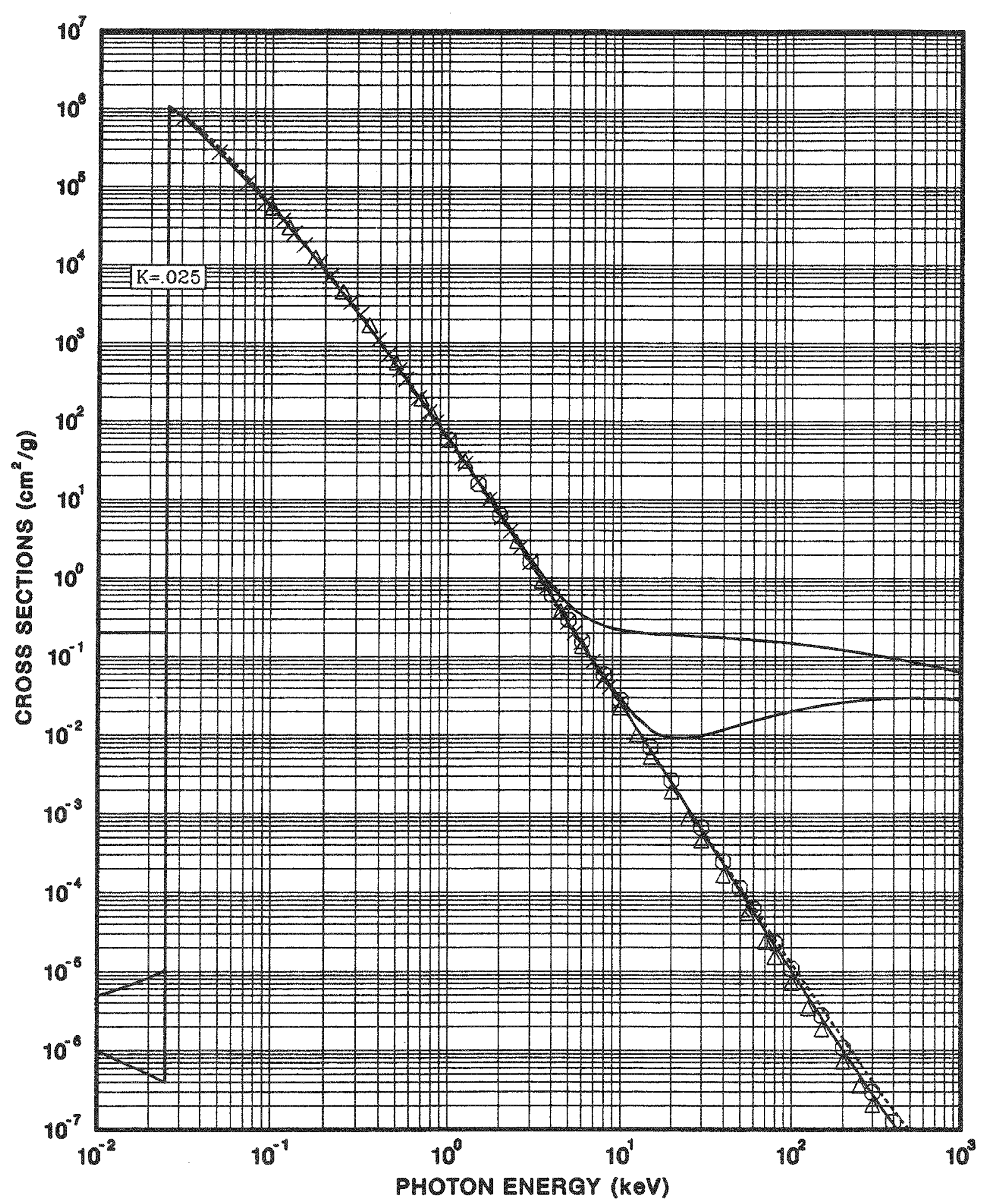

Barns/atom $=6.647 \times \mathrm{cm}^{2} / \mathrm{g}$ 


\section{LITHIUM 3}

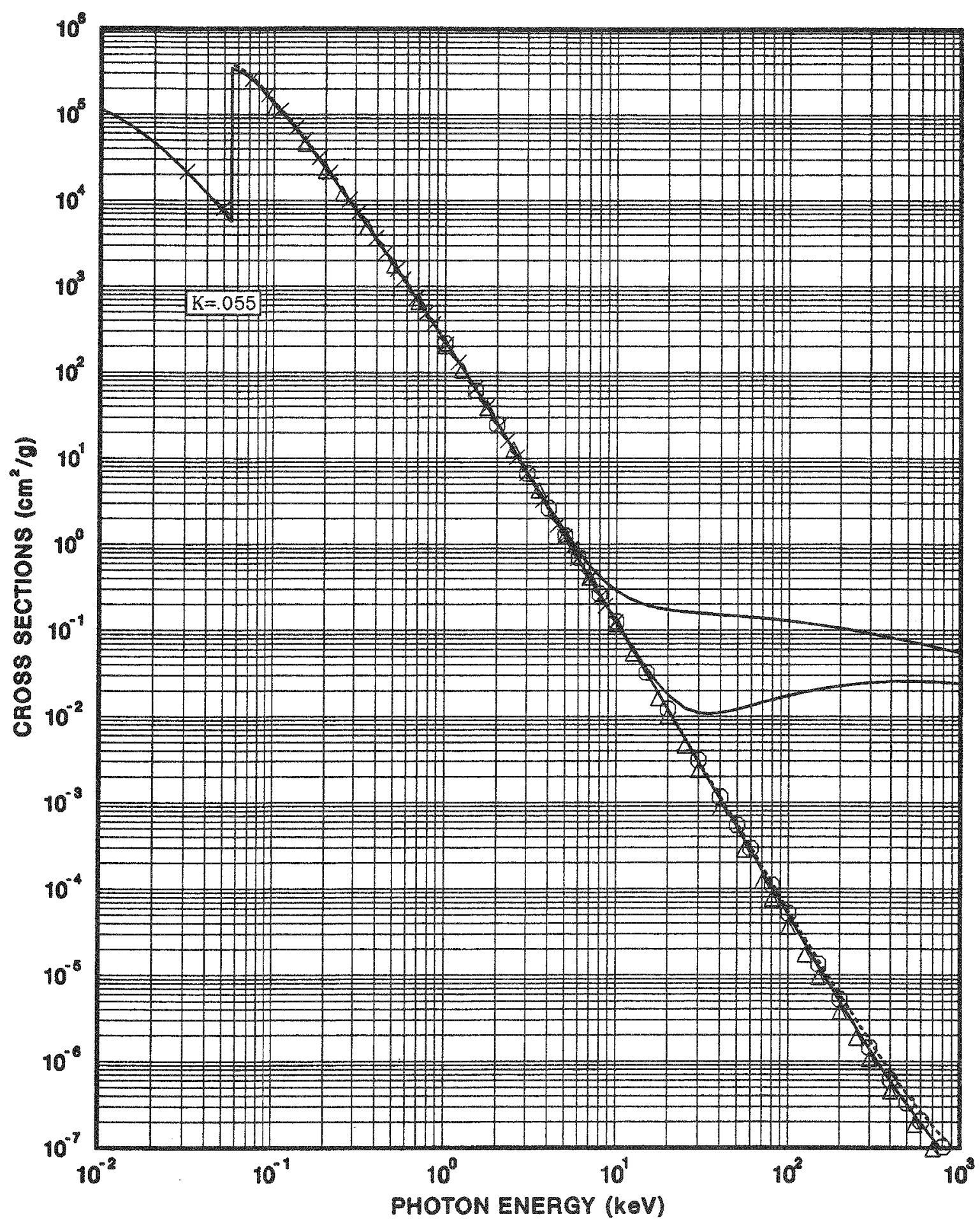

Barns/atom $=11.53 \times \mathrm{cm}^{2} / \mathrm{g}$ 


\section{BERYLLIUM 4}

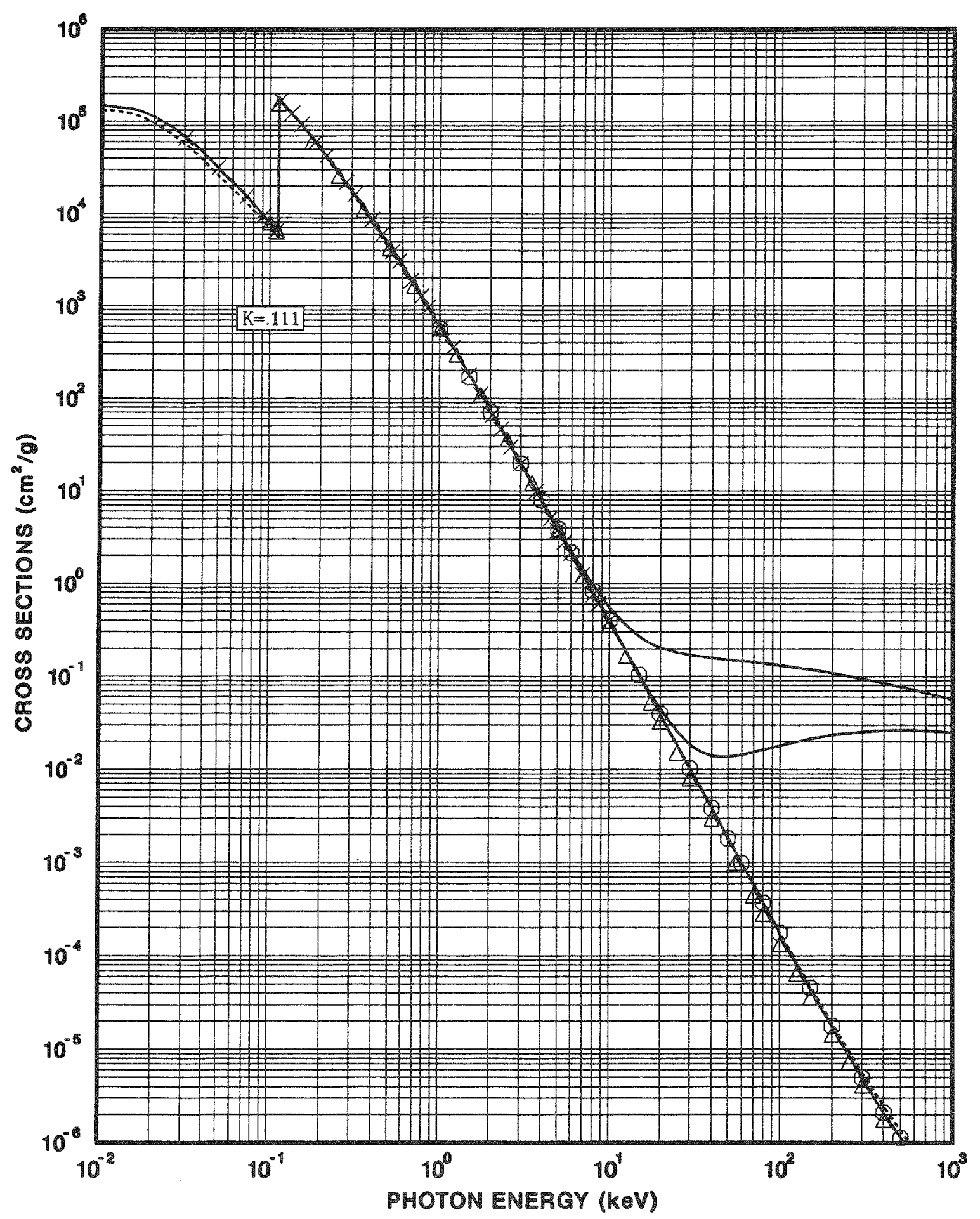

Barns/atom $=14.97 \times \mathrm{cm}^{2} / \mathrm{g}$ 


\section{BORON 5}

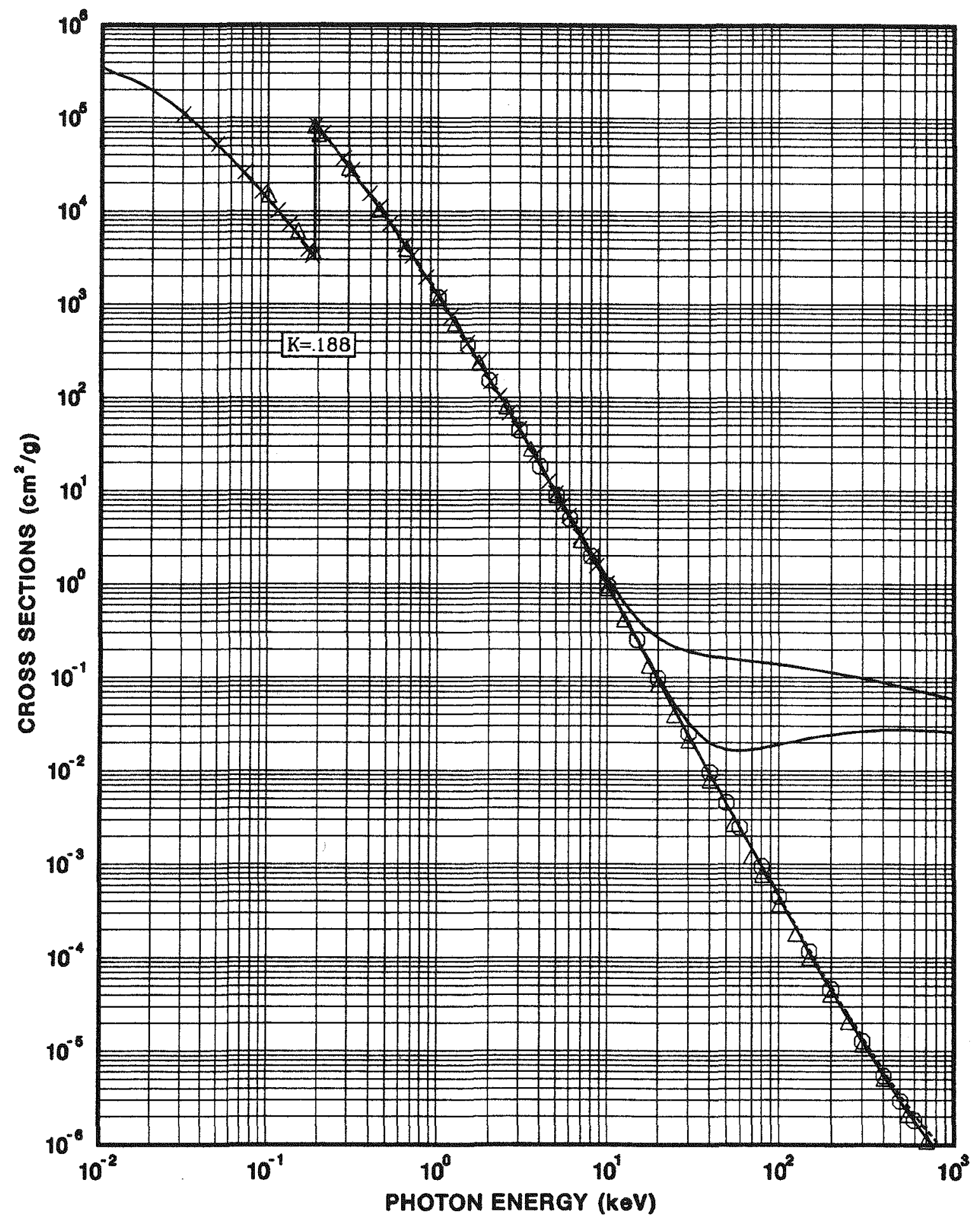

Barns/atom $=17.95 \times \mathrm{cm}^{2} / \mathrm{g}$ 
CARBON 6

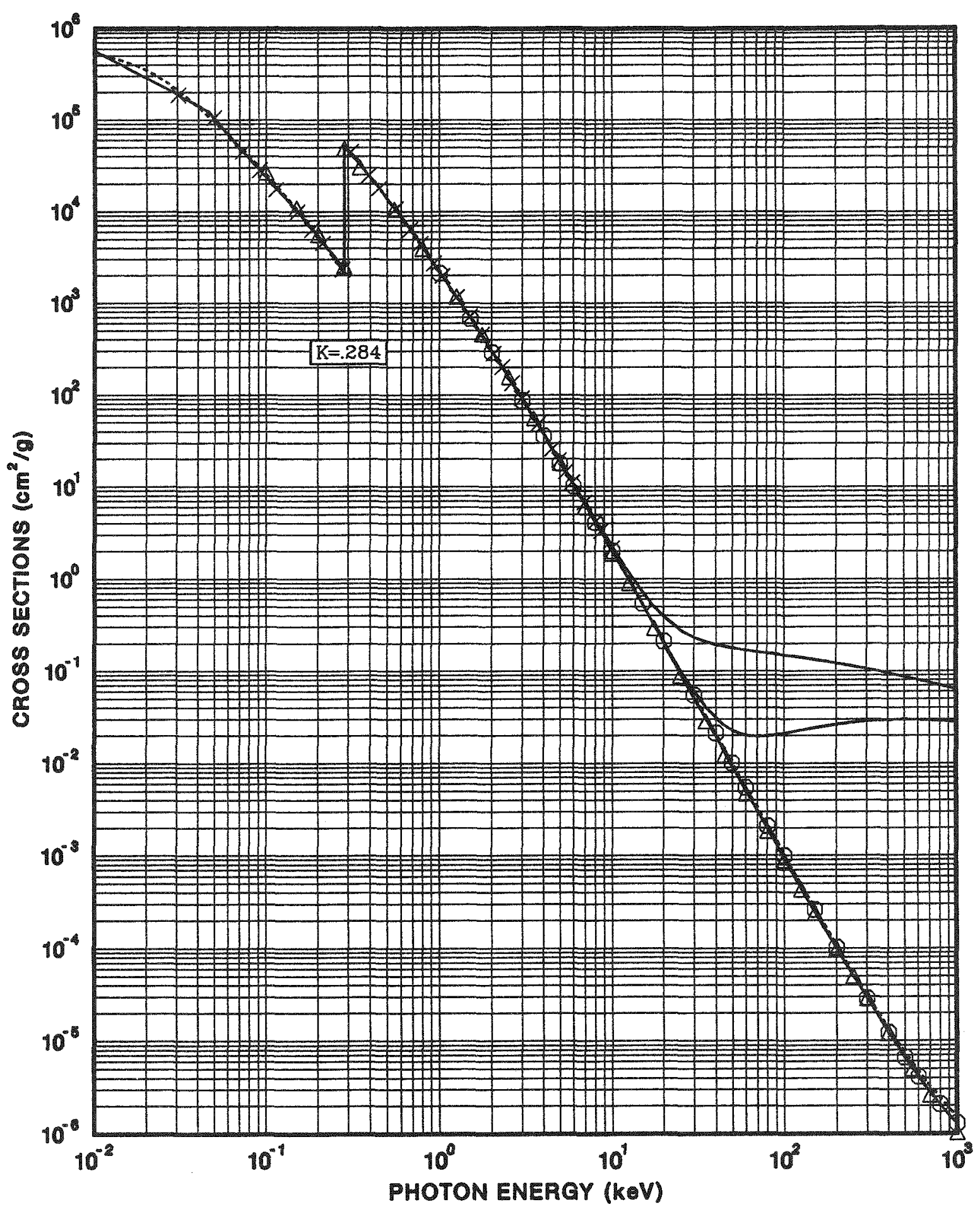

Barns/atom $=19.95 \times \mathrm{cm}^{2} / \mathrm{g}$ 


\section{NITROGEN 7}

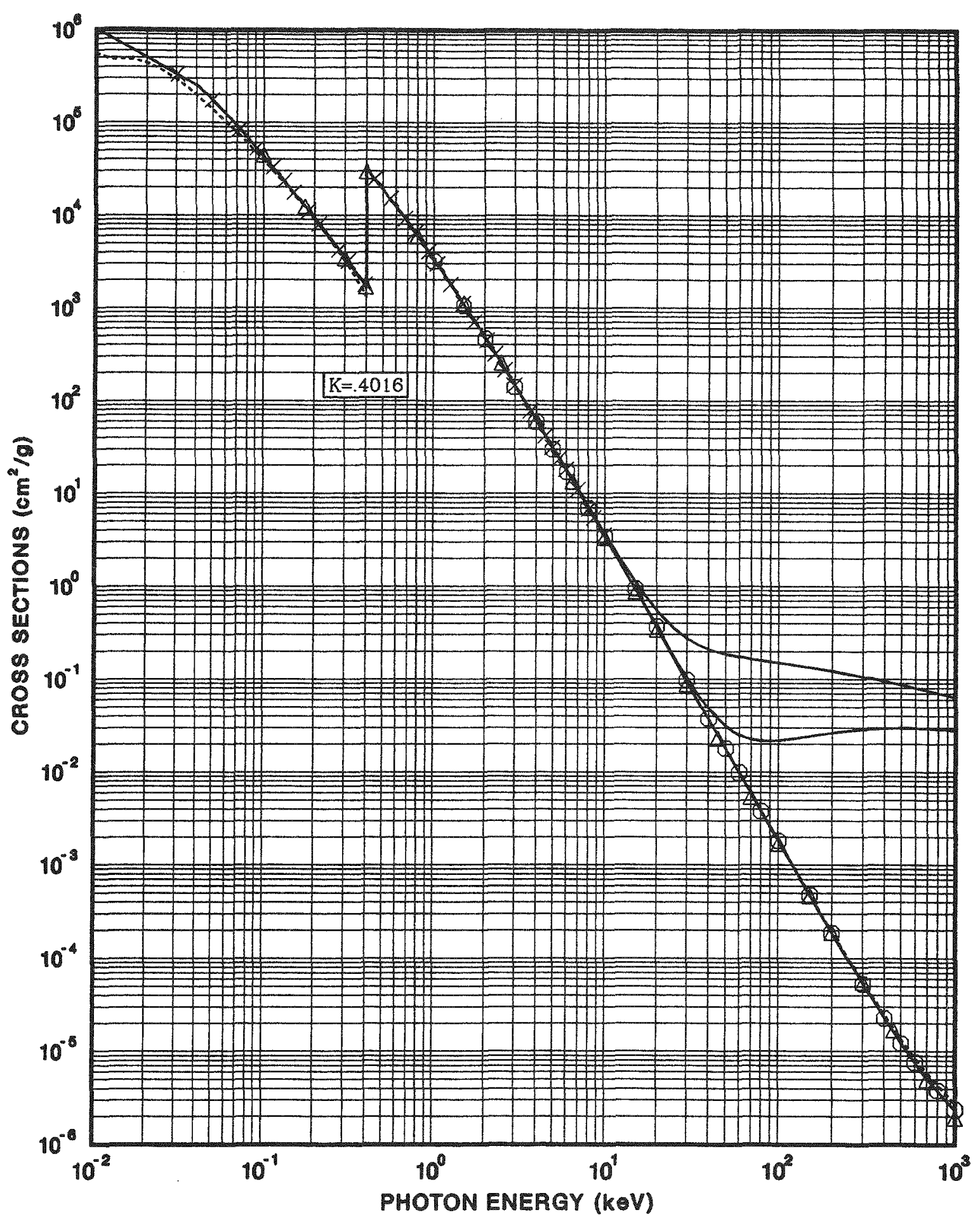

Barns/atom $=23.26 \times \mathrm{cm}^{2} / \mathrm{g}$ 
OXYGEN 8

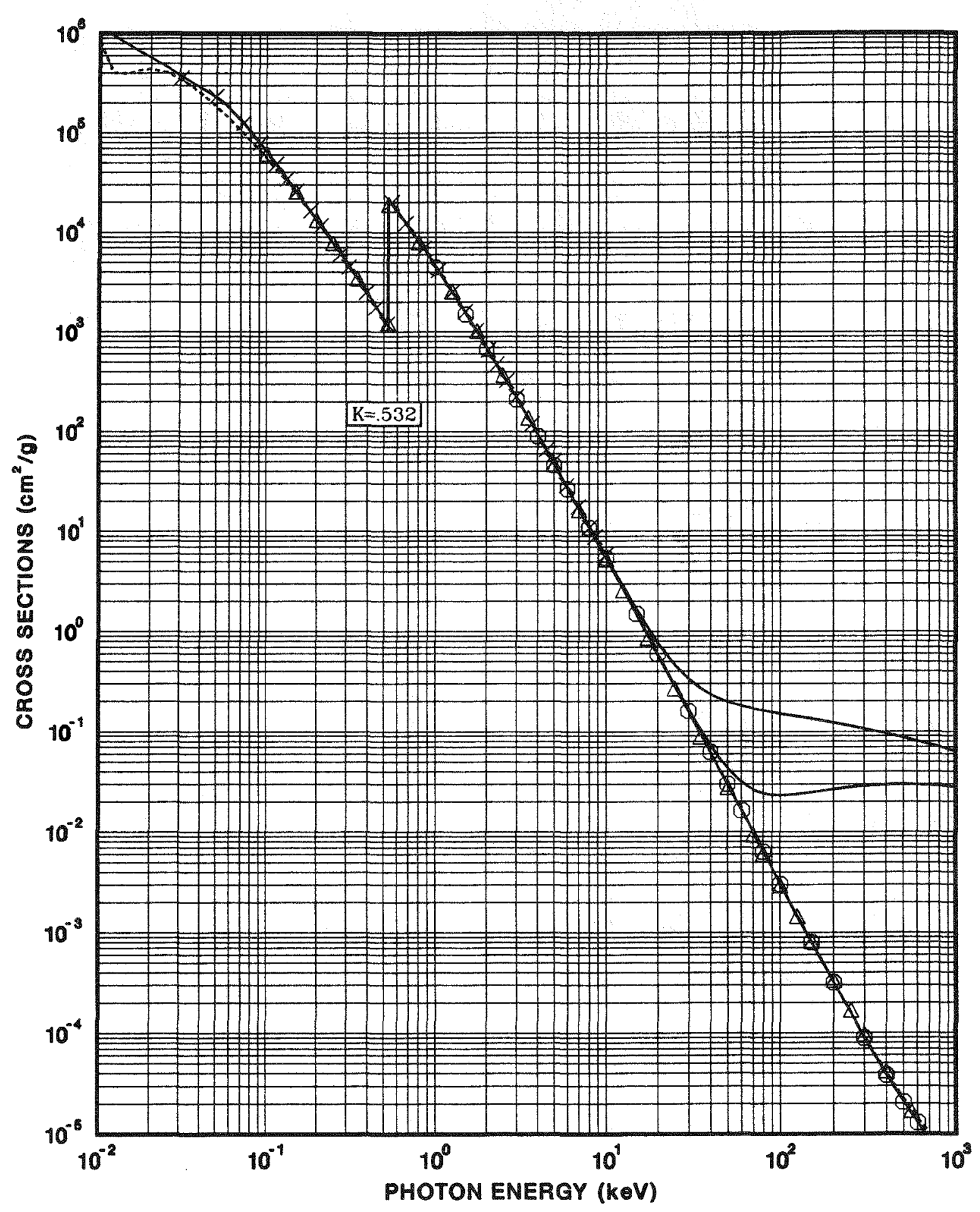

Barns $/$ atom $=26.57 \times \mathrm{cm}^{2} / \mathrm{g}$ 


\section{FLUORINE 9}

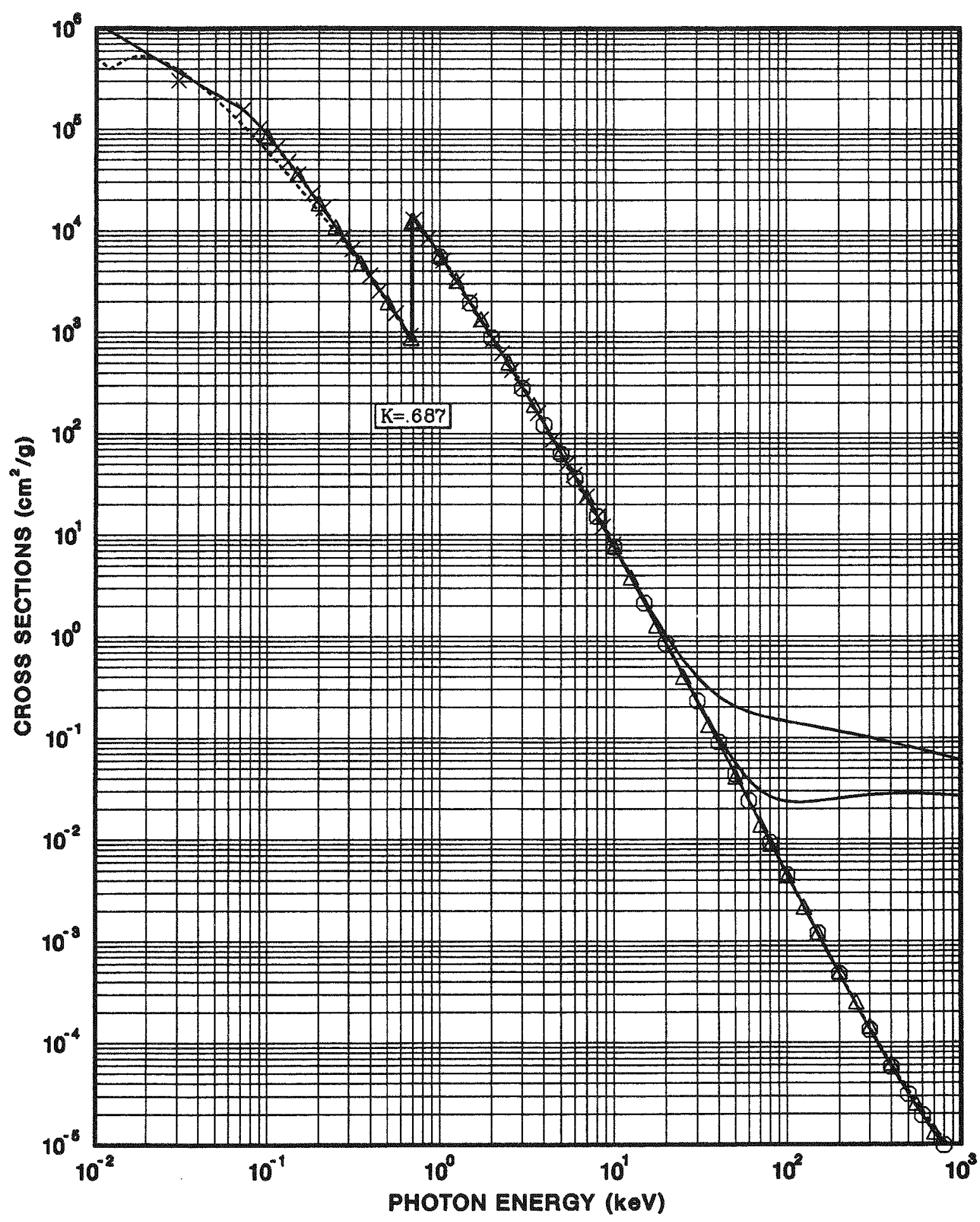

Barns $/$ atom $=31.55 \times \mathrm{cm}^{2} / \mathrm{g}$ 


\section{NEON 10}

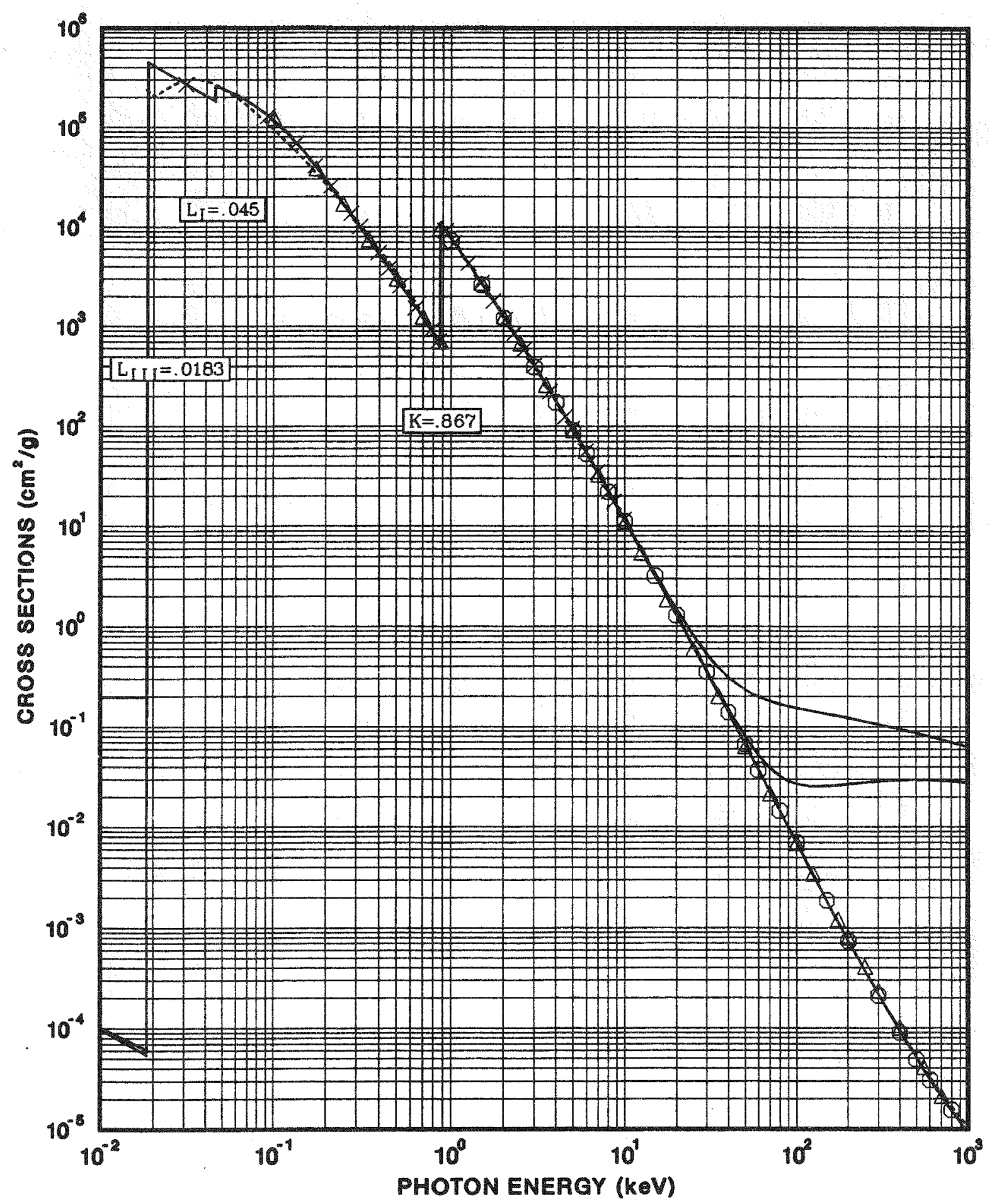

Barns/atom $=33.51 \times \mathrm{cm}^{2} / \mathrm{g}$ 
SODIUM 11

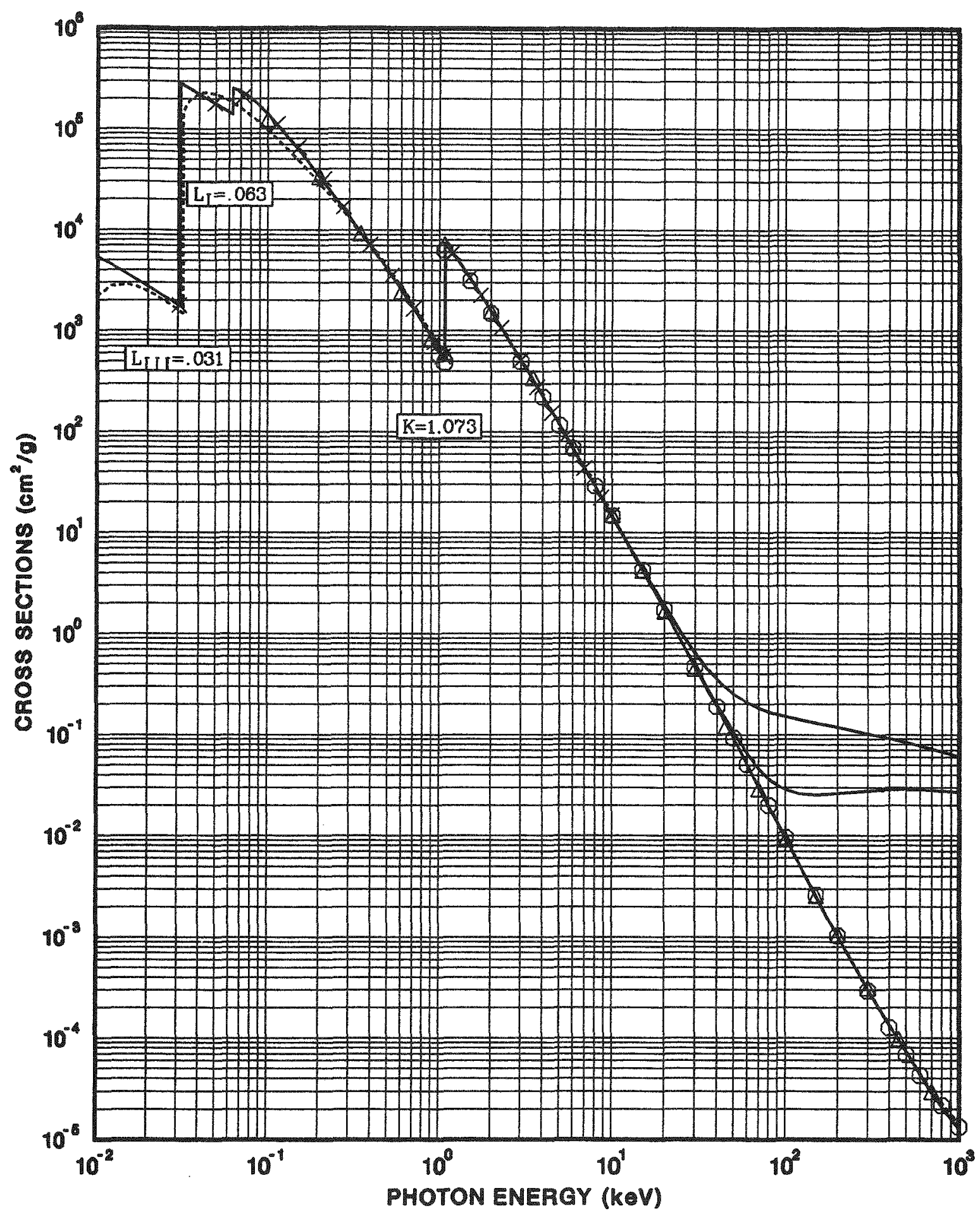

Barns/atom $=38.18 \times \mathrm{cm}^{2} / \mathrm{g}$ 
MAGNESIUM 12

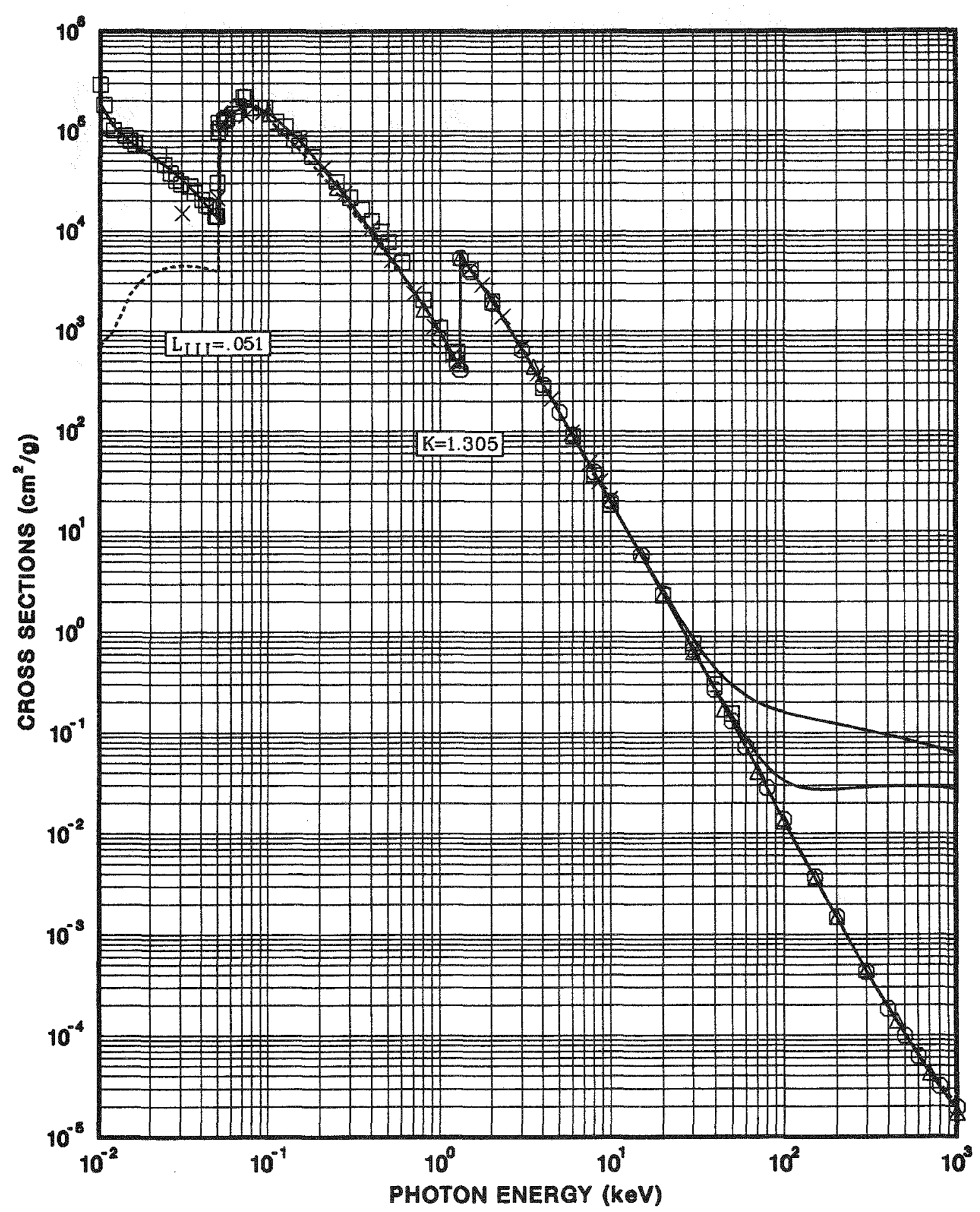

Barns $/$ atom $=40.36 \times \mathrm{cm}^{2} / \mathrm{g}$ 


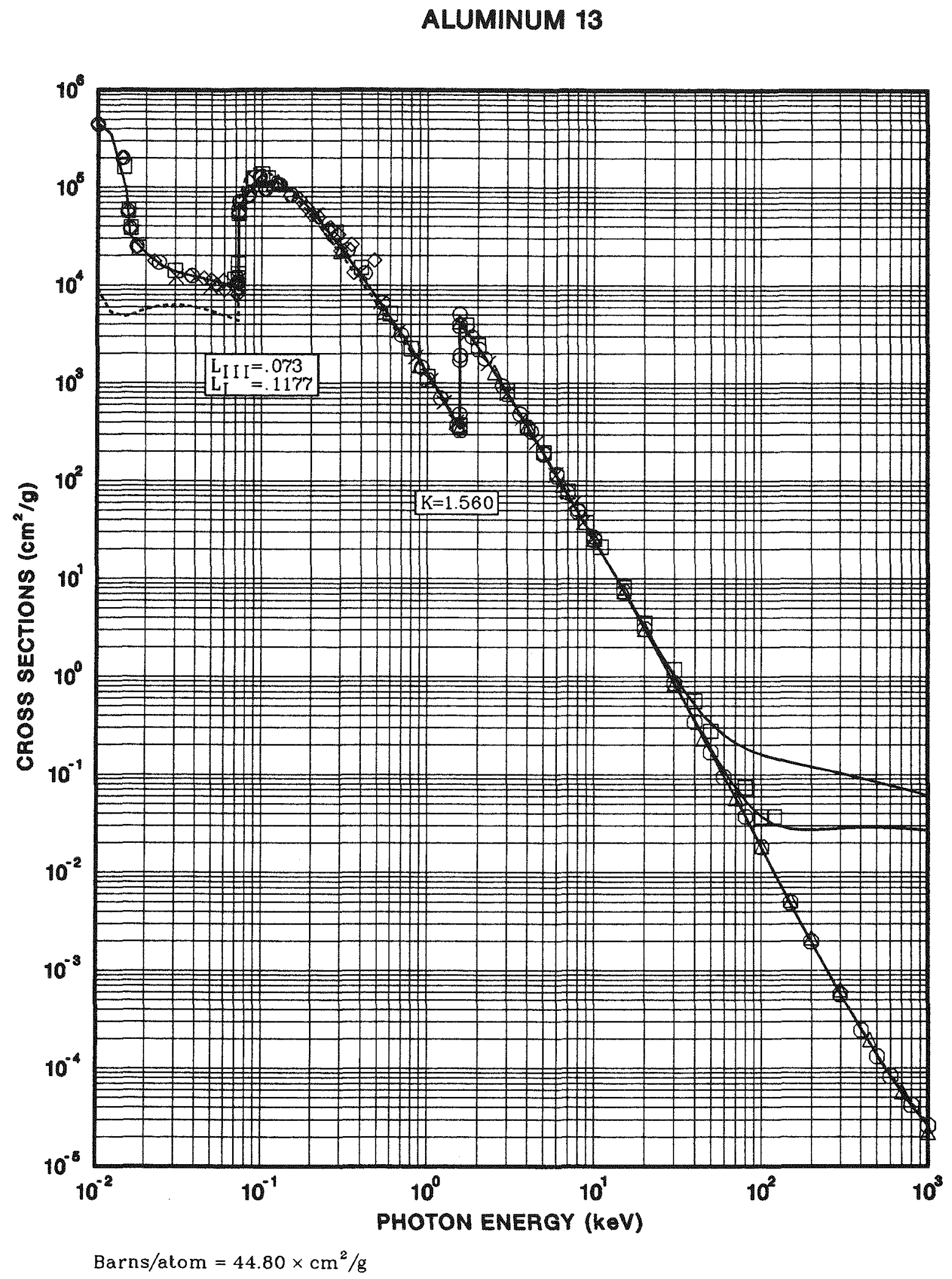




\section{SILICON 14}

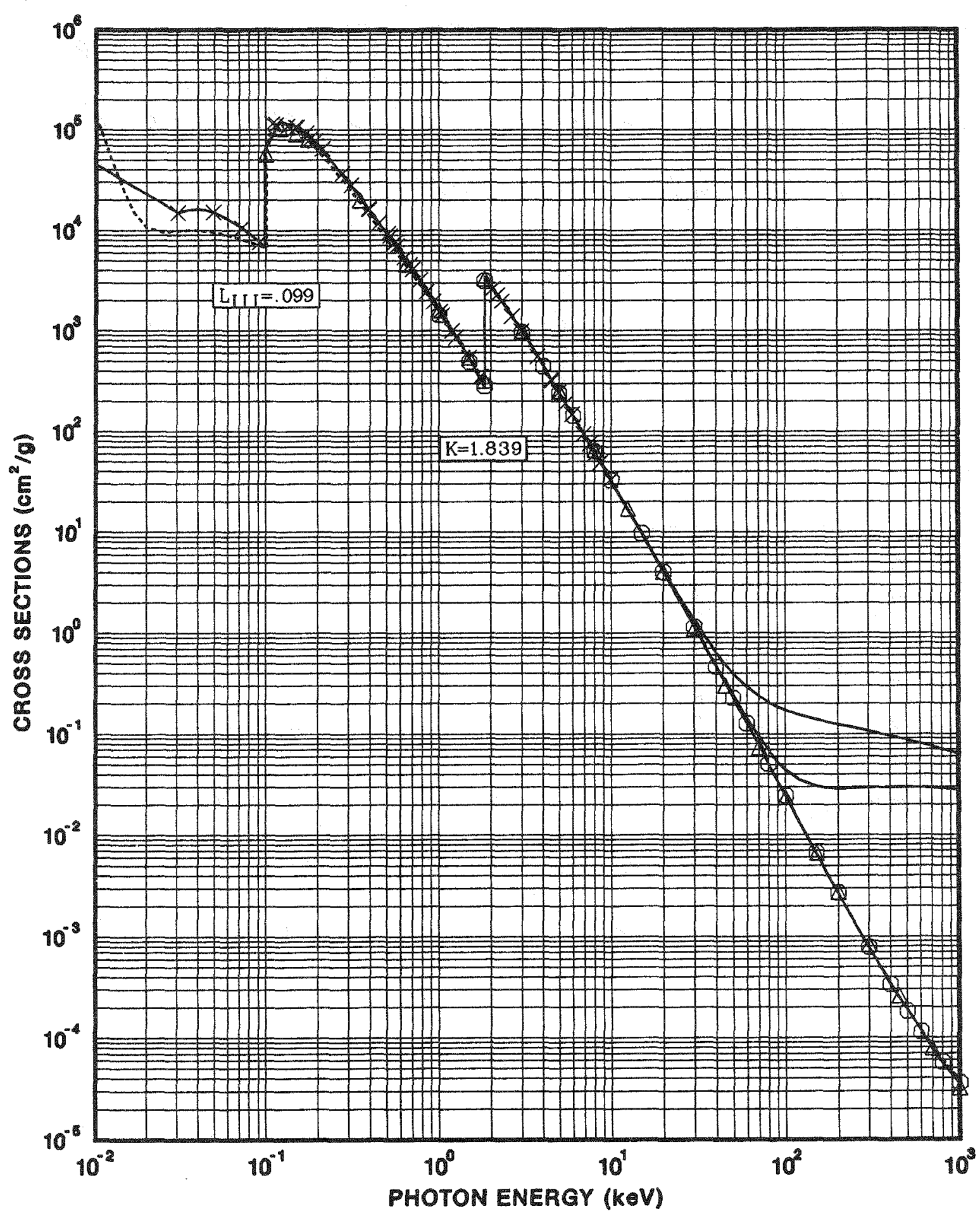

Barns/atom $=46.64 \times \mathrm{cm}^{2} / \mathrm{g}$ 


\section{PHOSPHORUS 15}

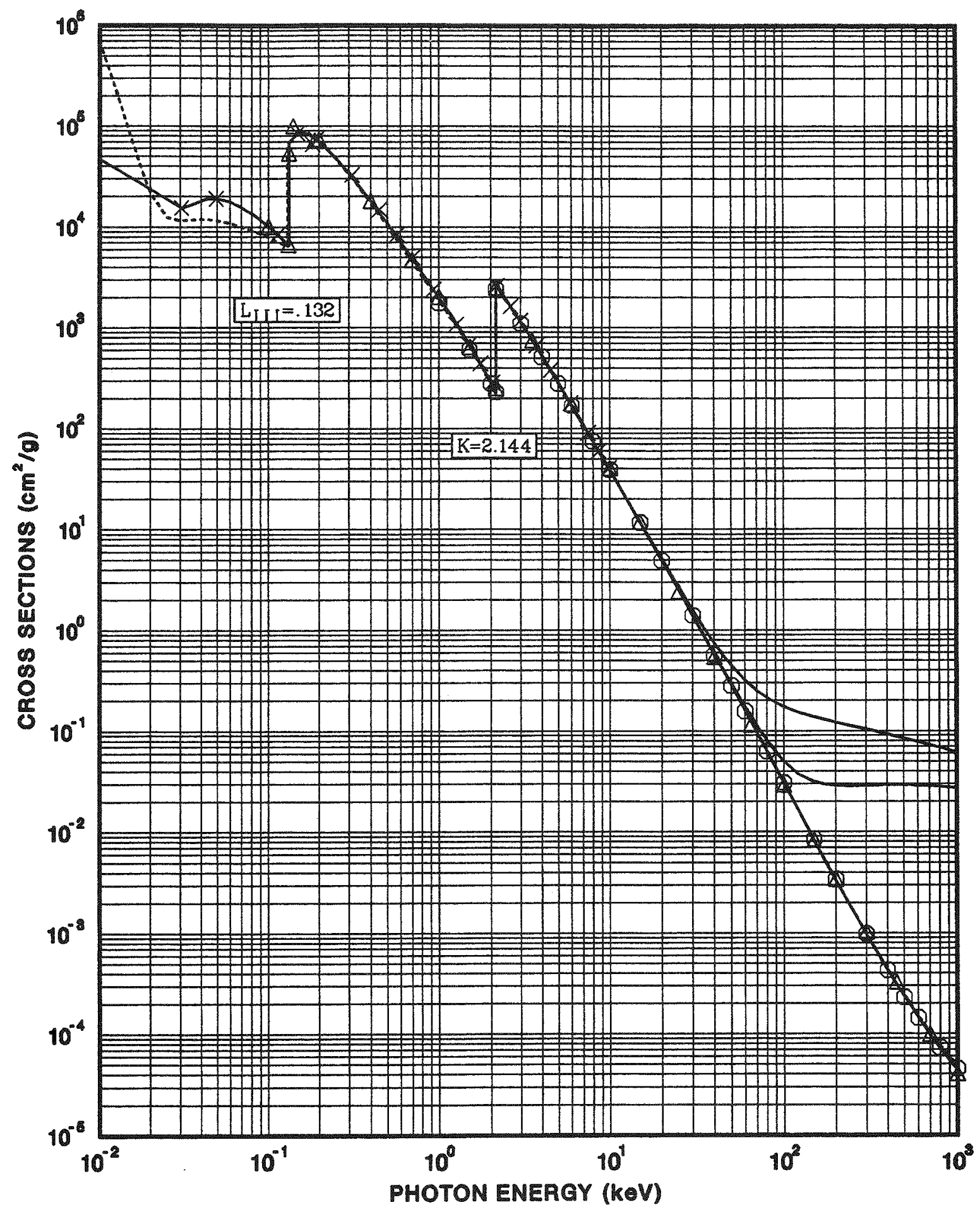

Barns/atom $=51.43 \times \mathrm{cm}^{2} / \mathrm{g}$ 


\section{SULFUR 16}

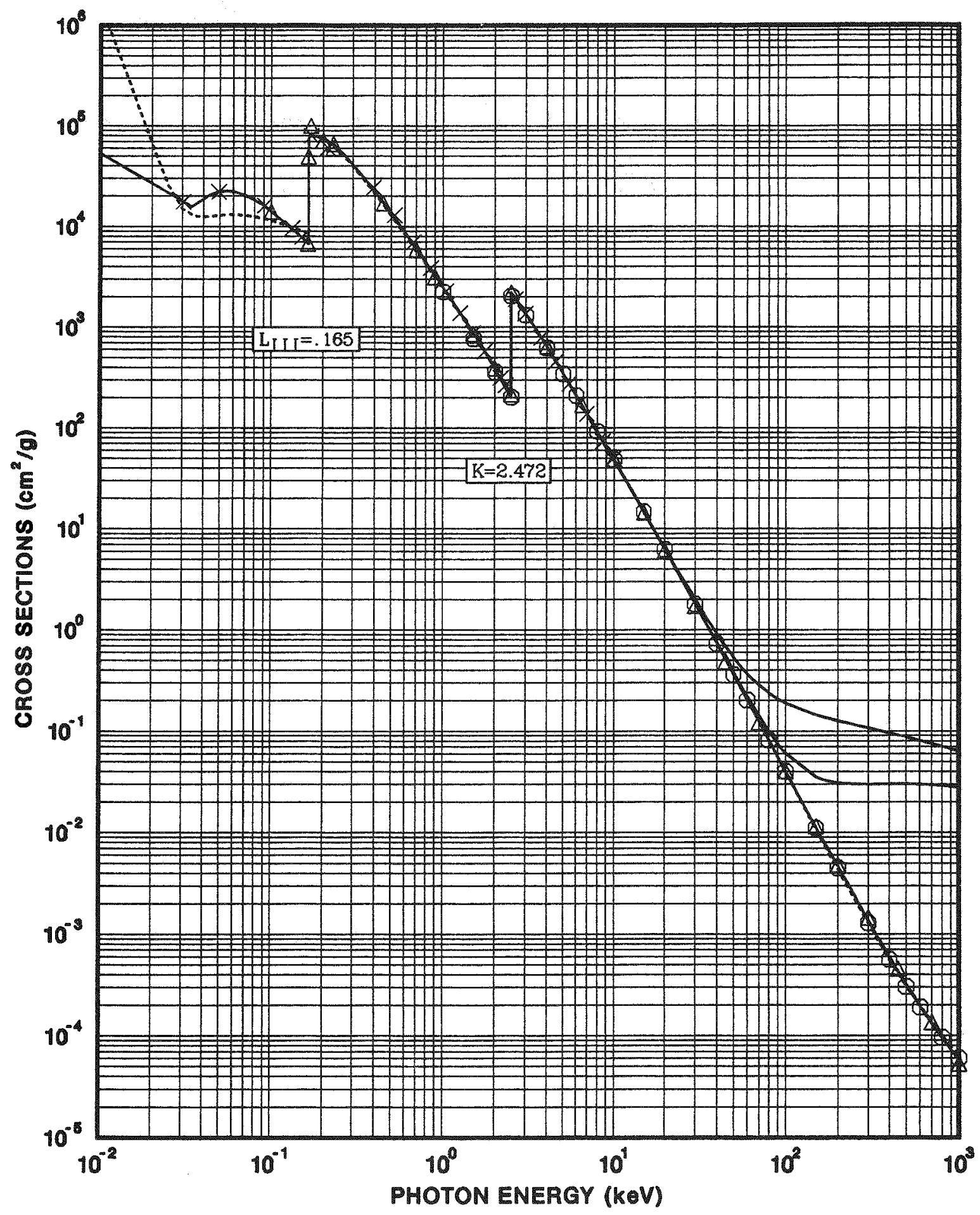

Barns $/$ atom $=53.24 \times \mathrm{cm}^{2} / \mathrm{g}$ 
CHLORINE 17

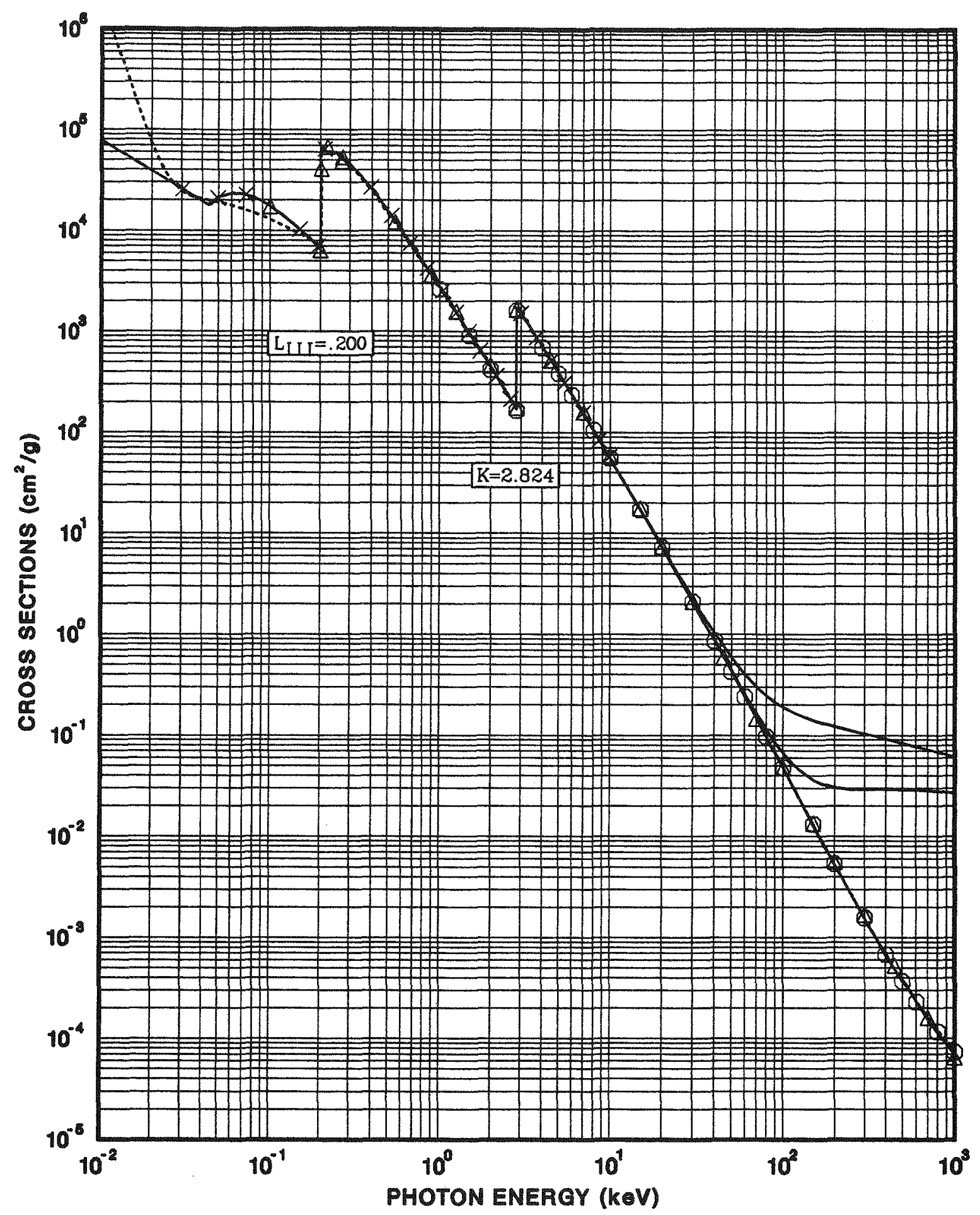

Barns/atom $=58.87 \times \mathrm{cm}^{2} / \mathrm{g}$ 


\section{ARGON 18}

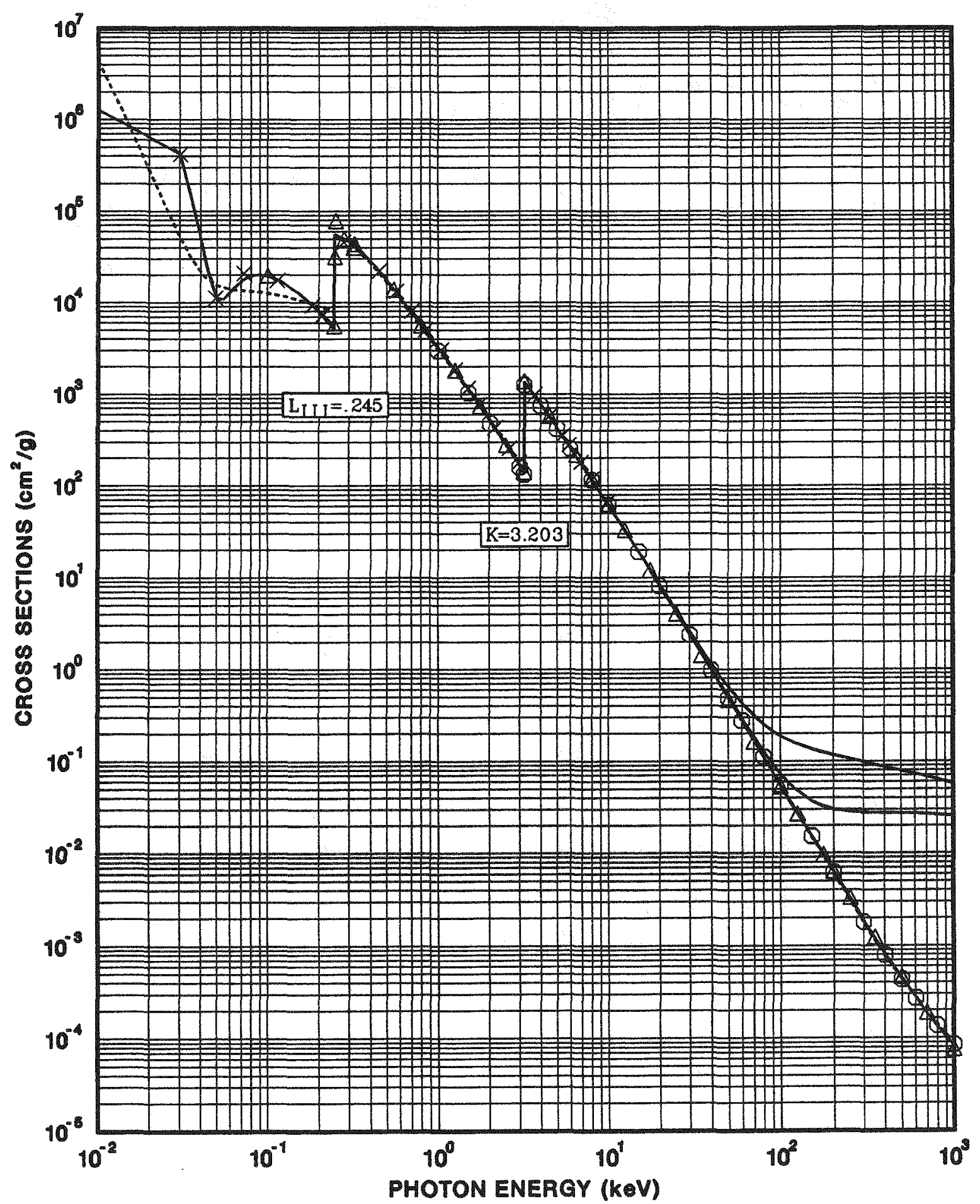

Barns/atom $=66.34 \times \mathrm{cm}^{2} / \mathrm{g}$ 
POTASSIUM 19

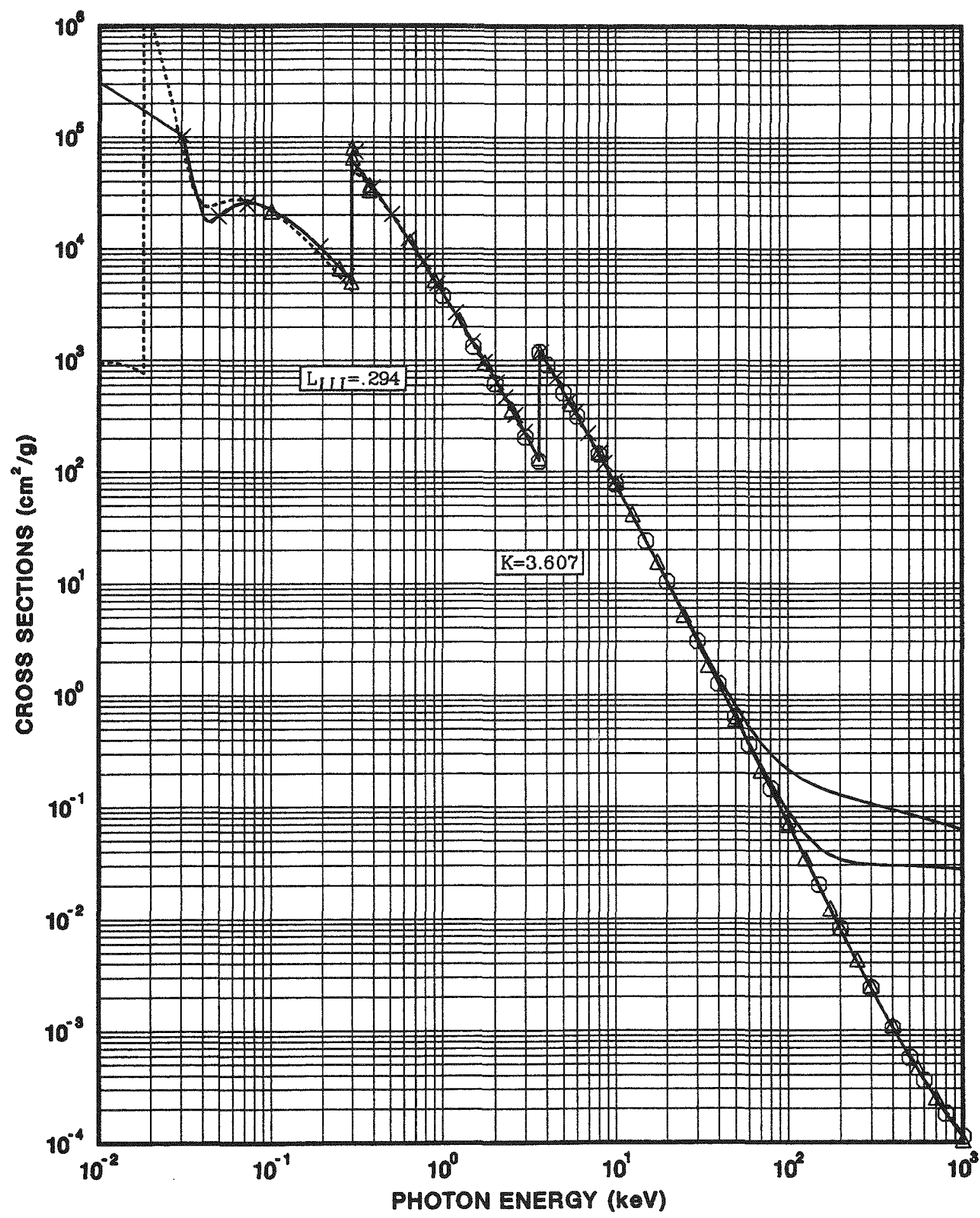

Barns $/$ atom $=64.93 \times \mathrm{cm}^{2} / \mathrm{g}$ 


\section{CALCIUM 20}

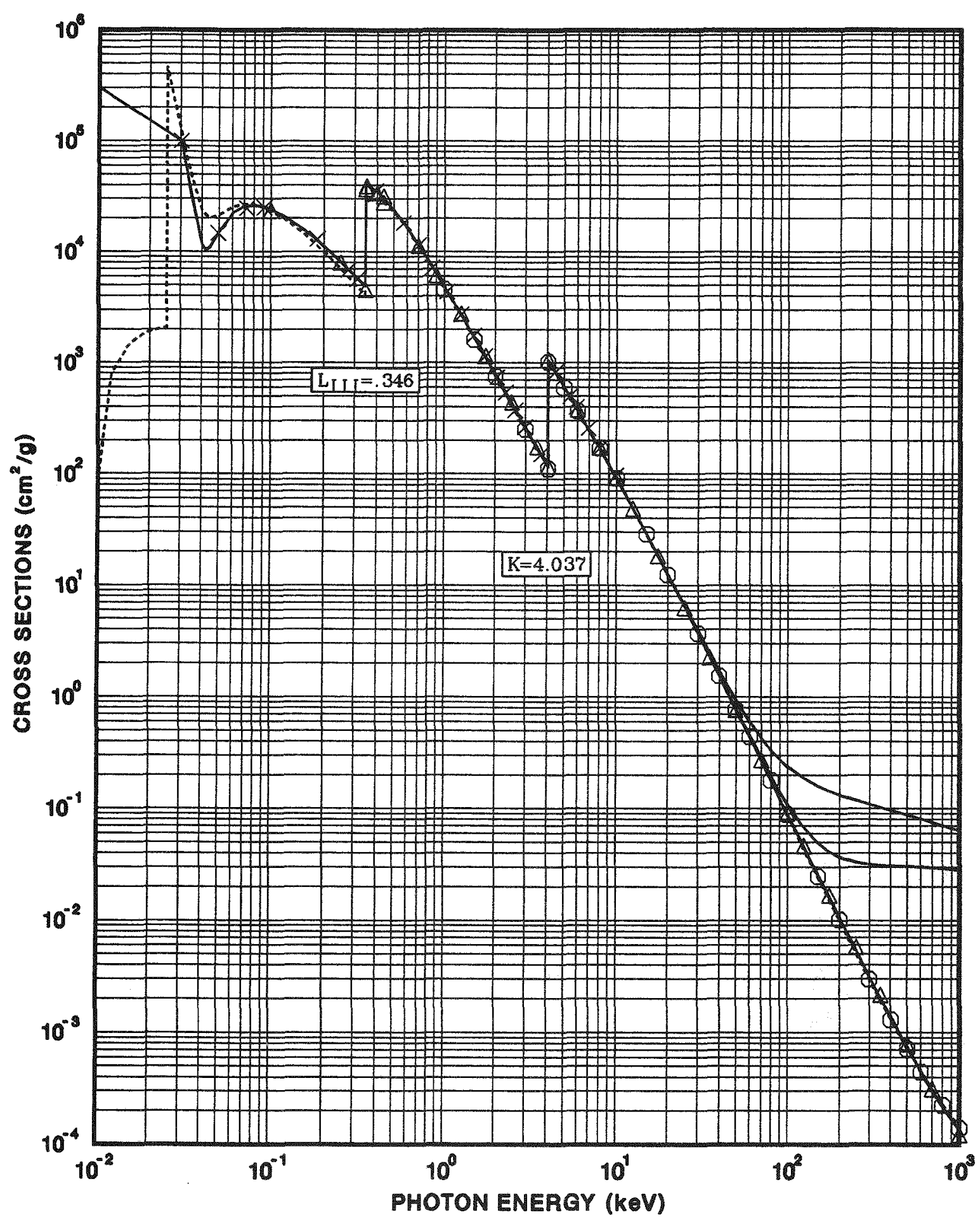

Barns/atom $=66.56 \times \mathrm{cm}^{2} / \mathrm{g}$ 
SCANDIUM 21

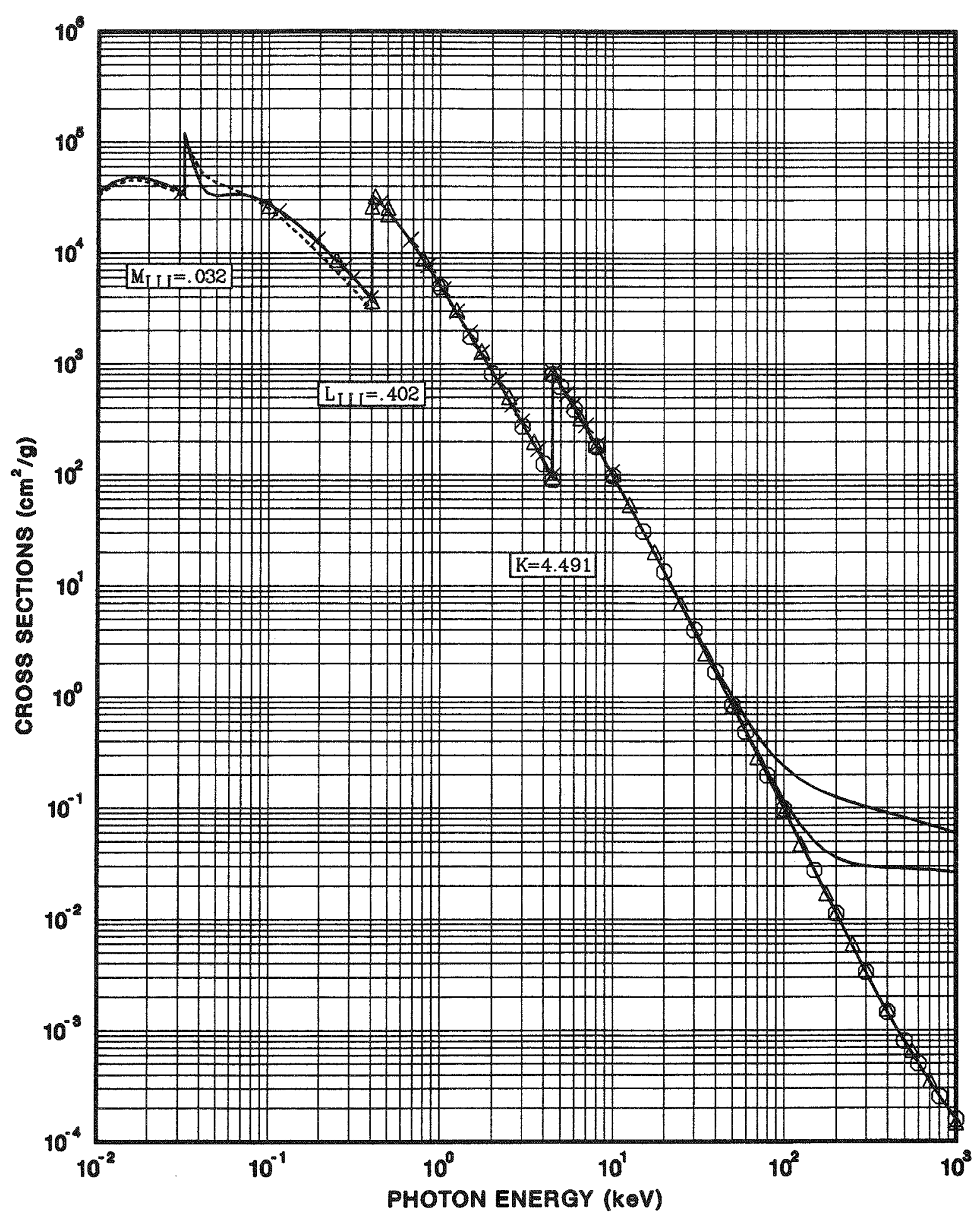

Barns/atom $=74.65 \times \mathrm{cm}^{2} / \mathrm{g}$ 
TITANIUM 22

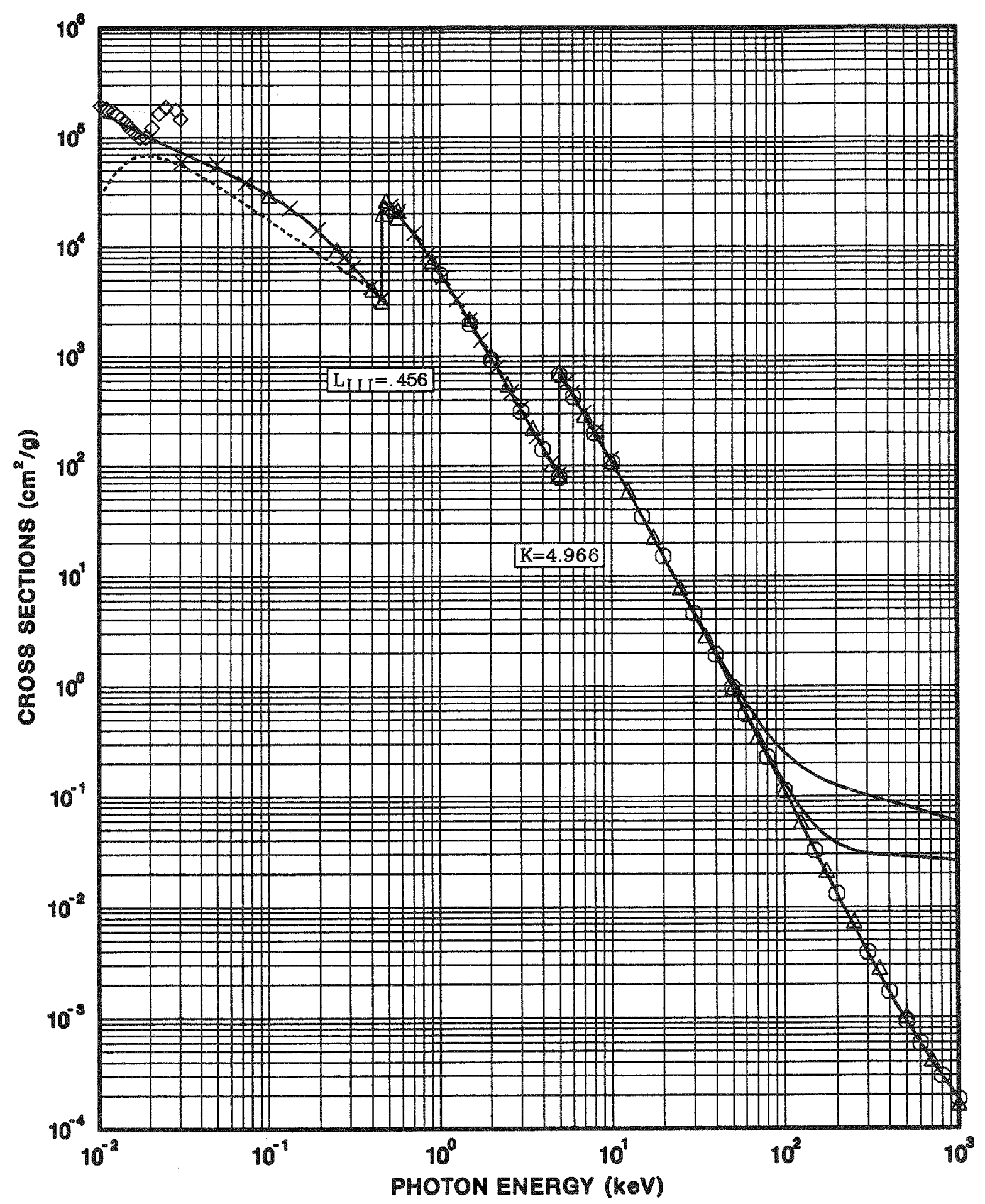

Barns/atom $=79.51 \times \mathrm{cm}^{2} / \mathrm{g}$ 
VANADIUM 23

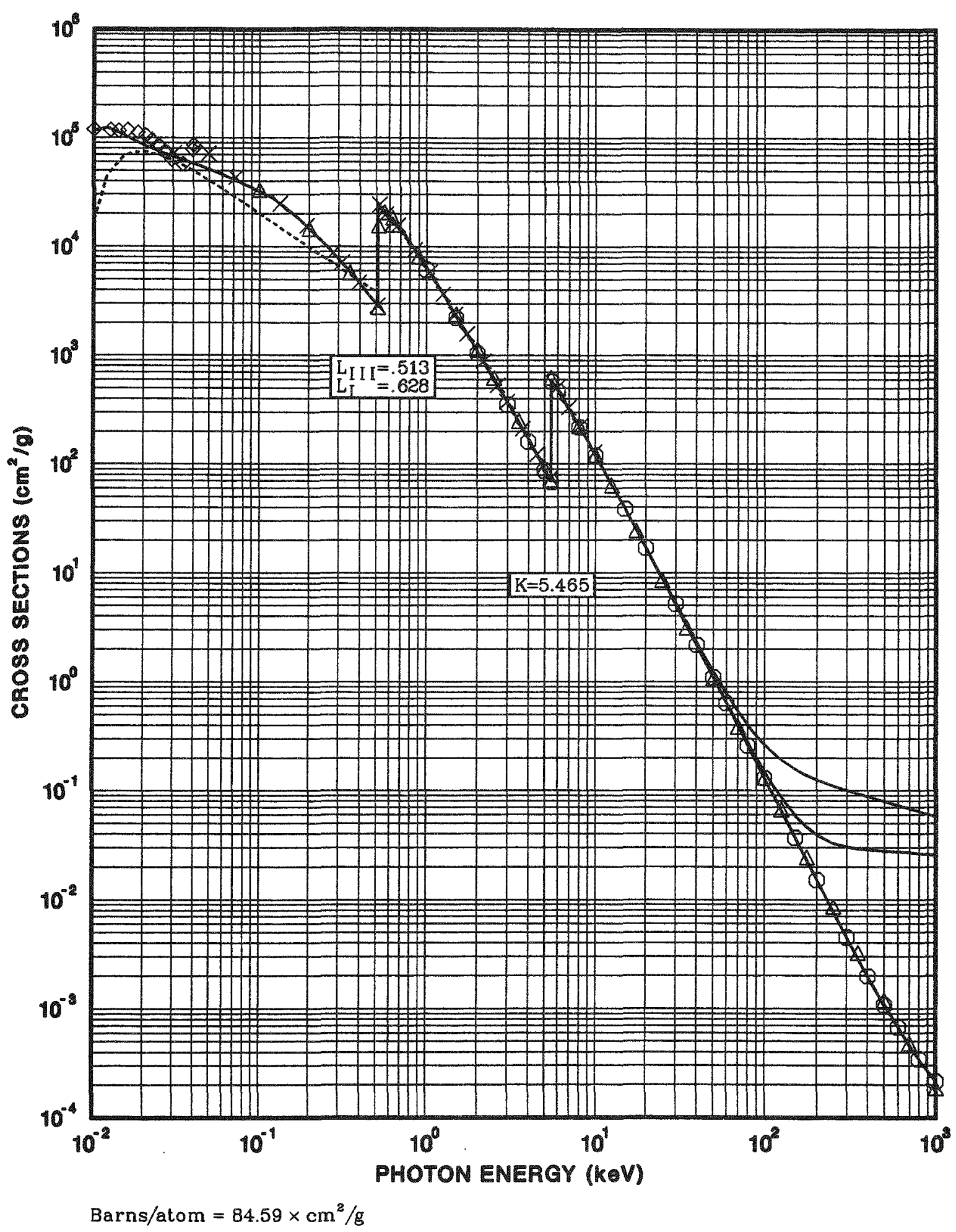


CHROMIUM 24

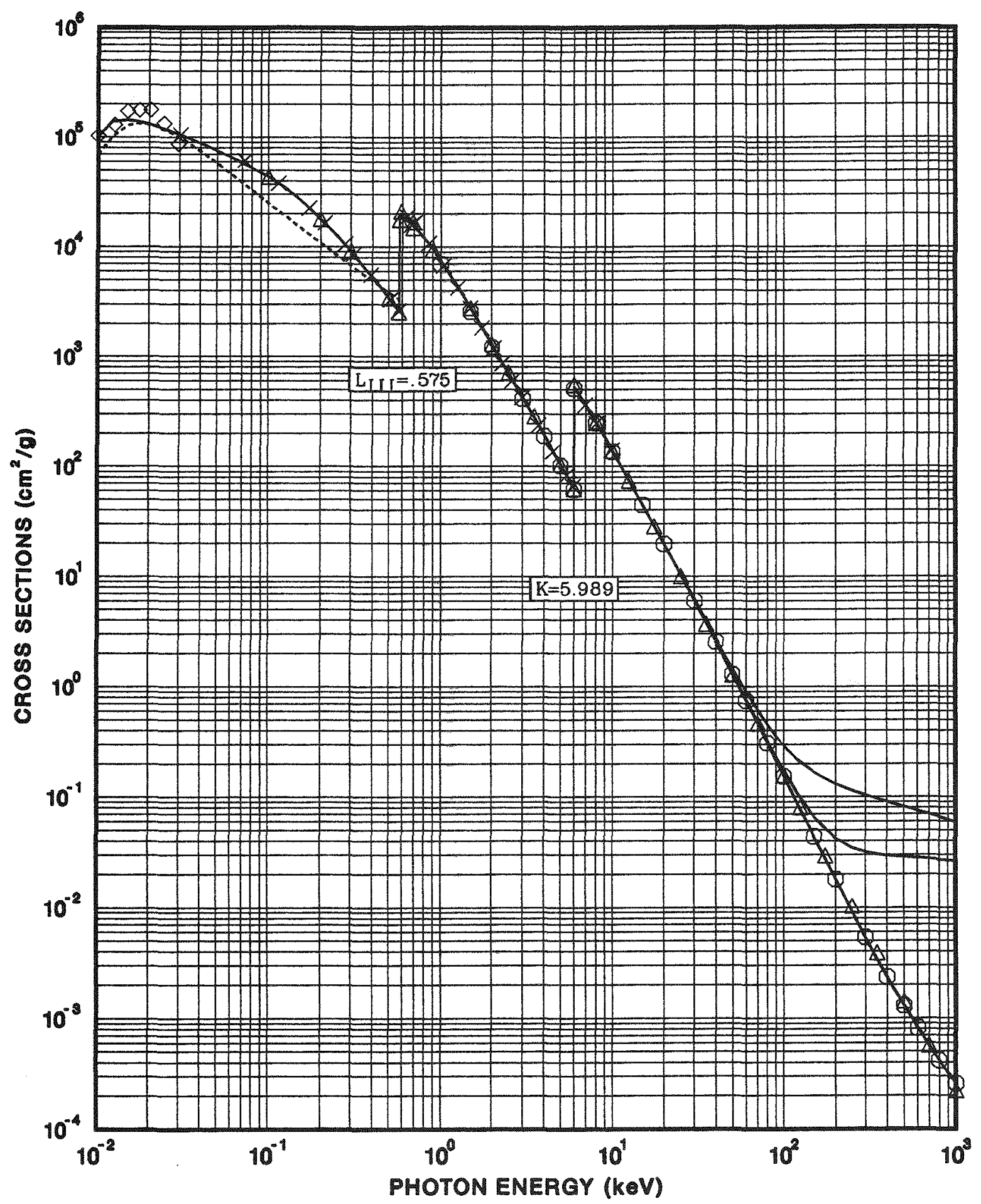

Barns/atom $=86.34 \times \mathrm{cm}^{2} / \mathrm{g}$ 
MANGANESE 25

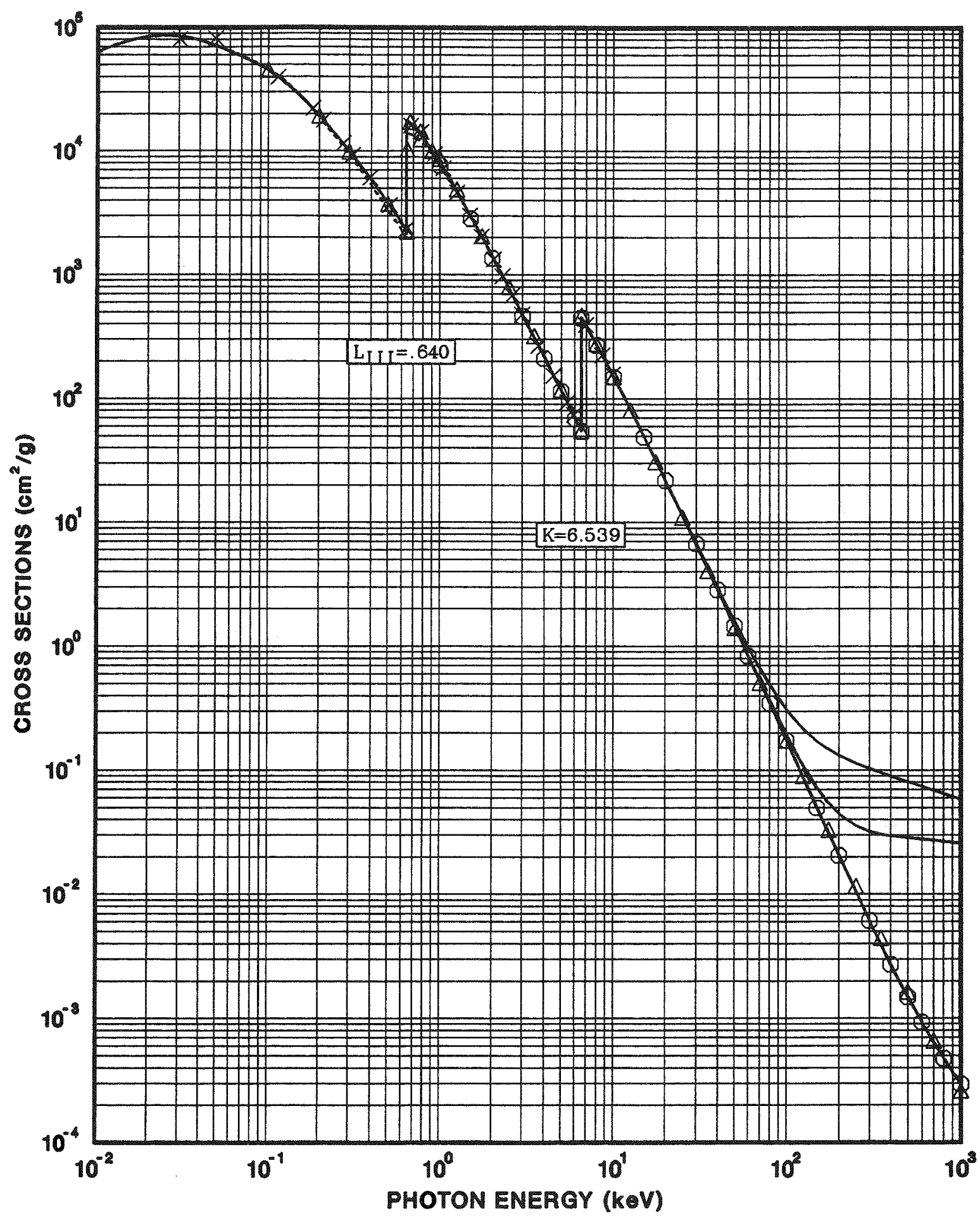

Barns/atom $=91.23 \times \mathrm{cm}^{2} / \mathrm{g}$ 
IRON 26

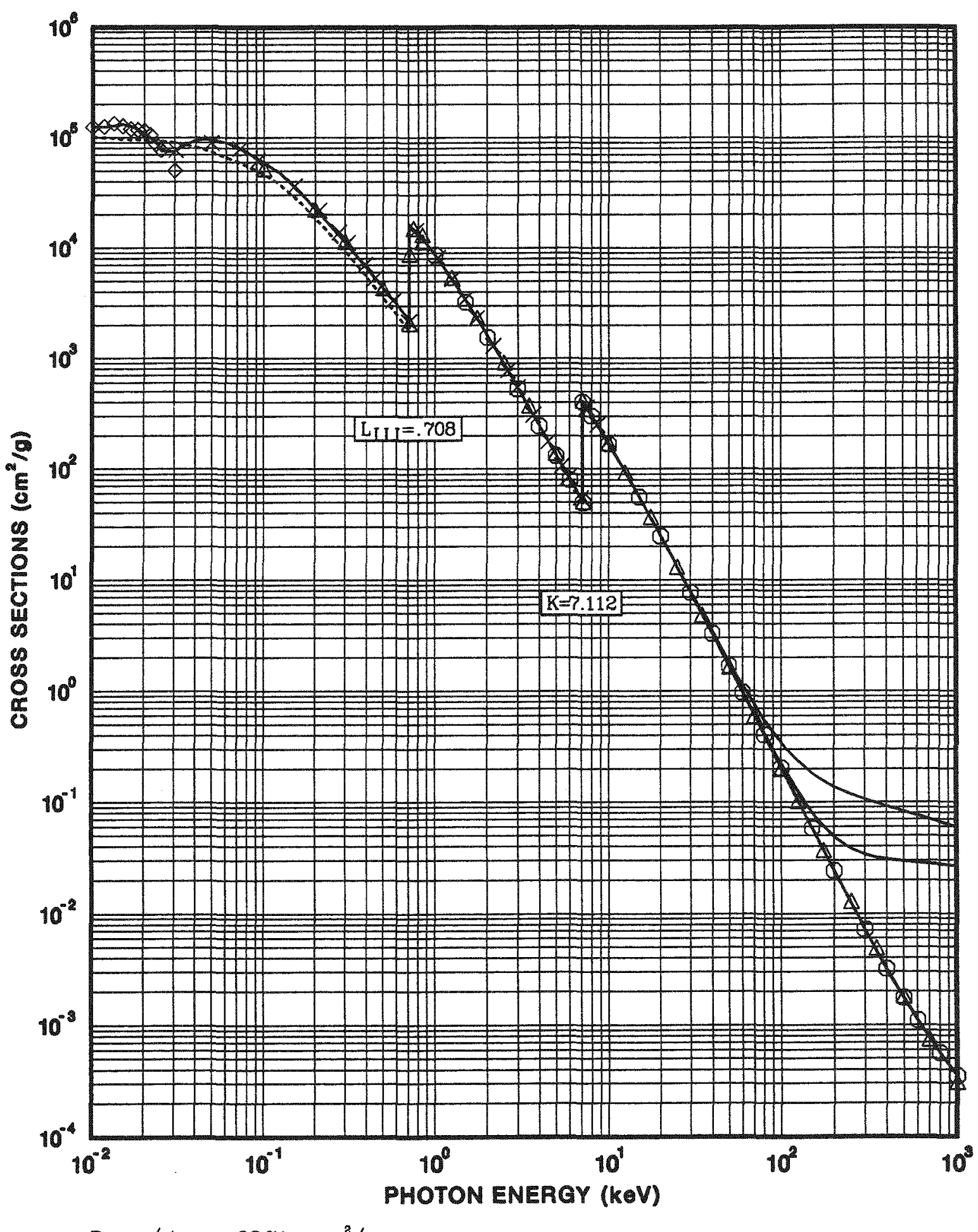

Barns/atom $=92.74 \times \mathrm{cm}^{2} / \mathrm{g}$ 
COBALT 27

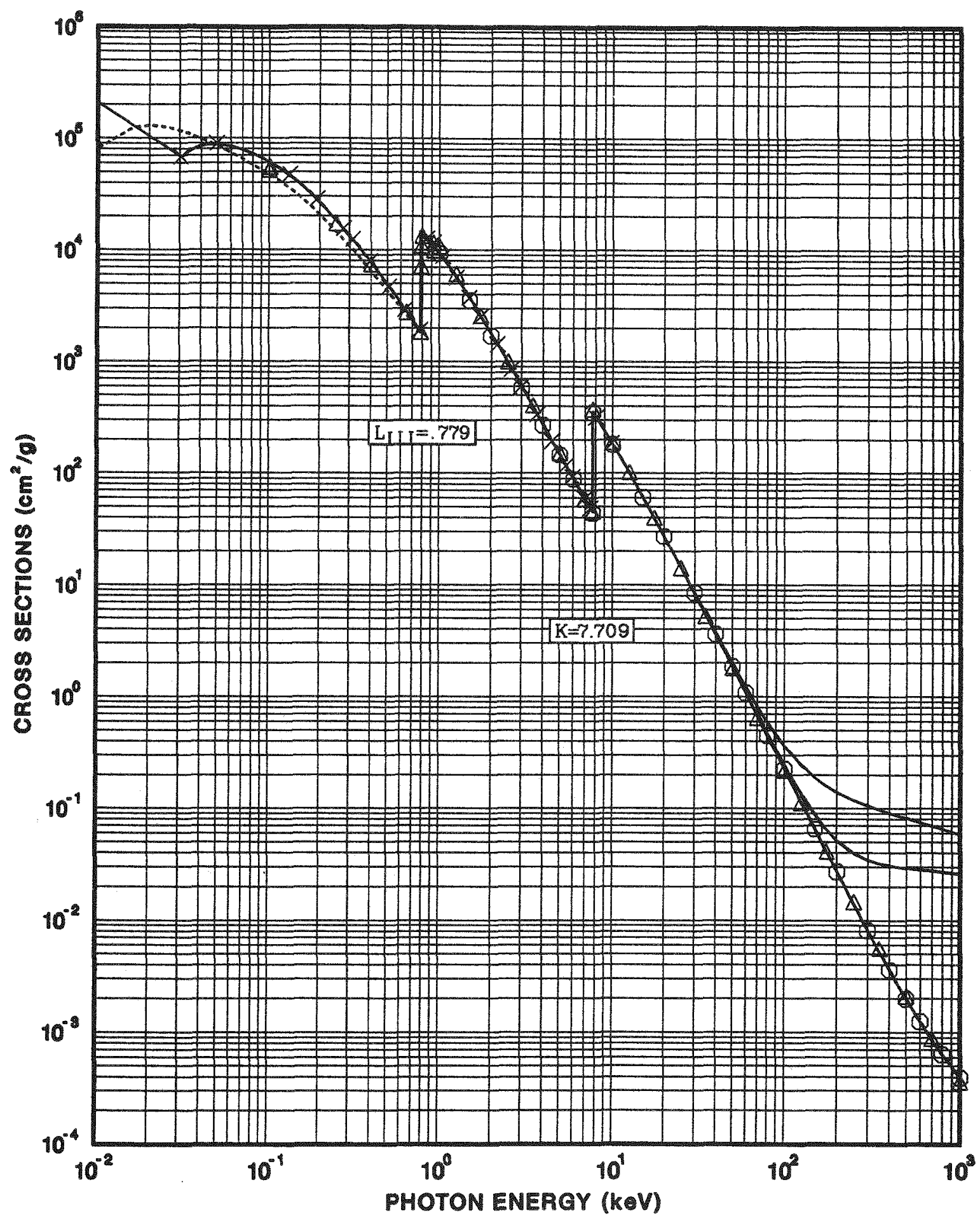

Barns/atom $=97.86 \times \mathrm{cm}^{2} / \mathrm{g}$ 
NICKEL 28

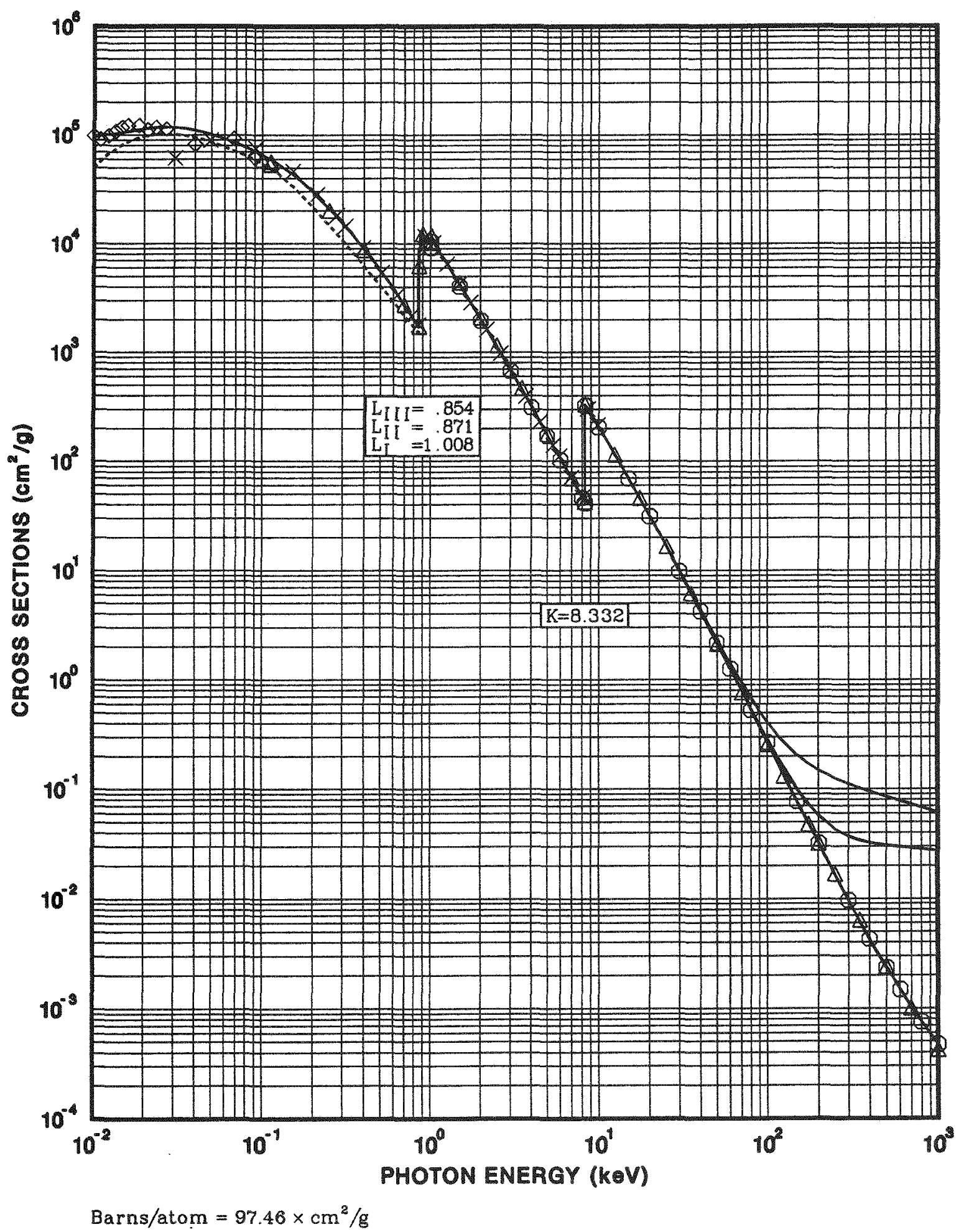


COPPER 29

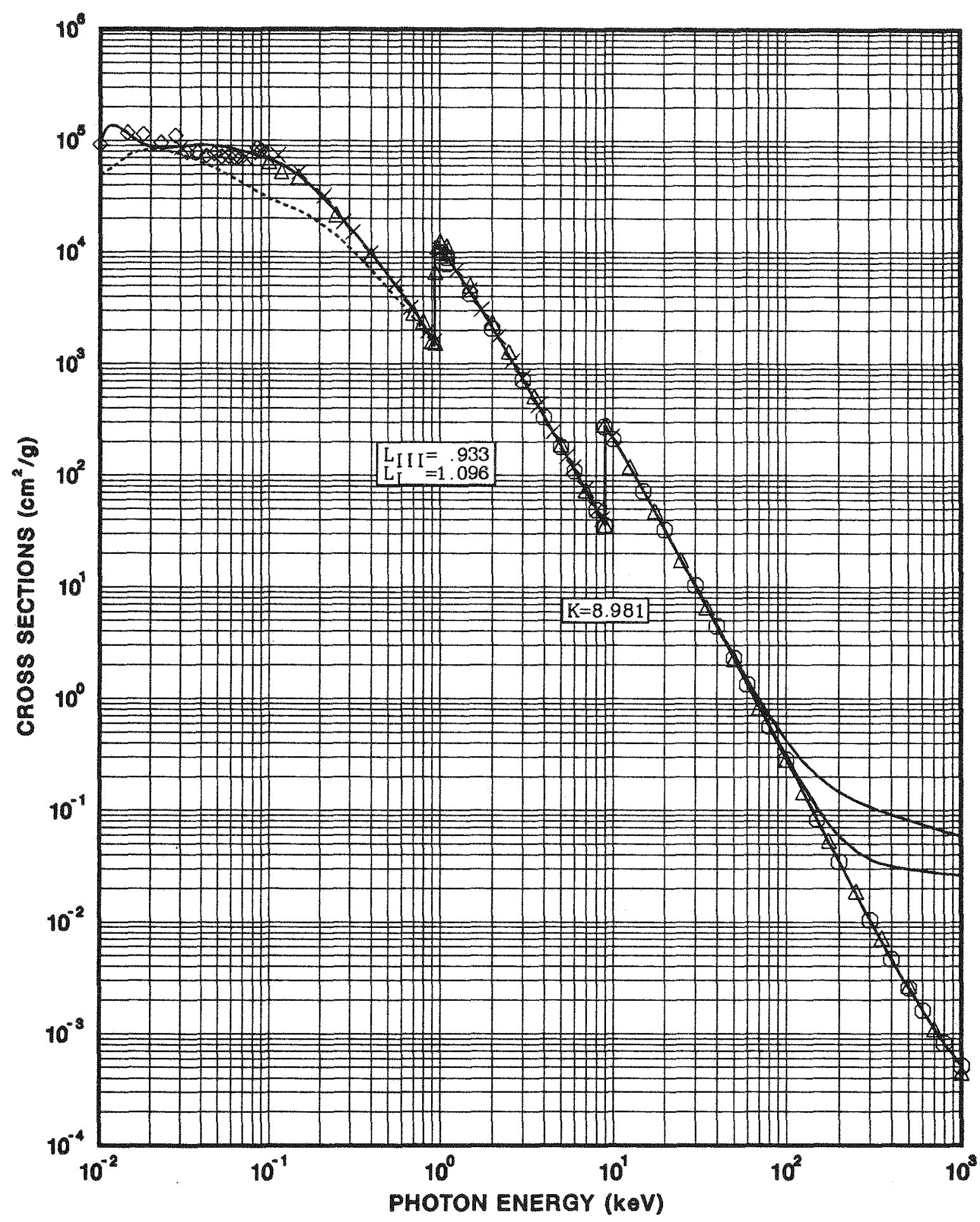

Barns $/ \mathrm{atom}=105.5 \times \mathrm{cm}^{2} / \mathrm{g}$ 


\section{ZINC 30}

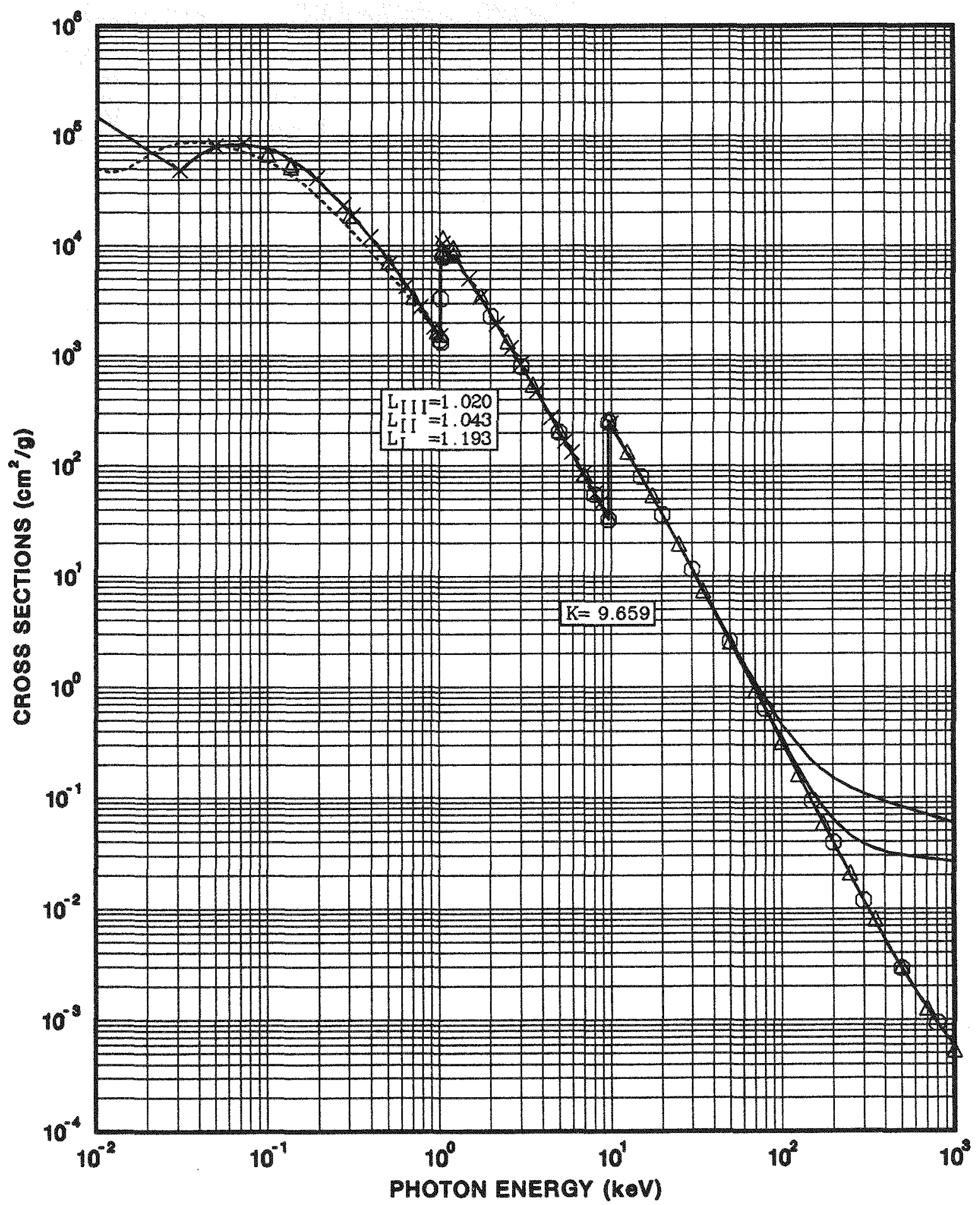

Barns/atom $=108.6 \times \mathrm{cm}^{2} / \mathrm{g}$ 


\section{GALLIUM 31}

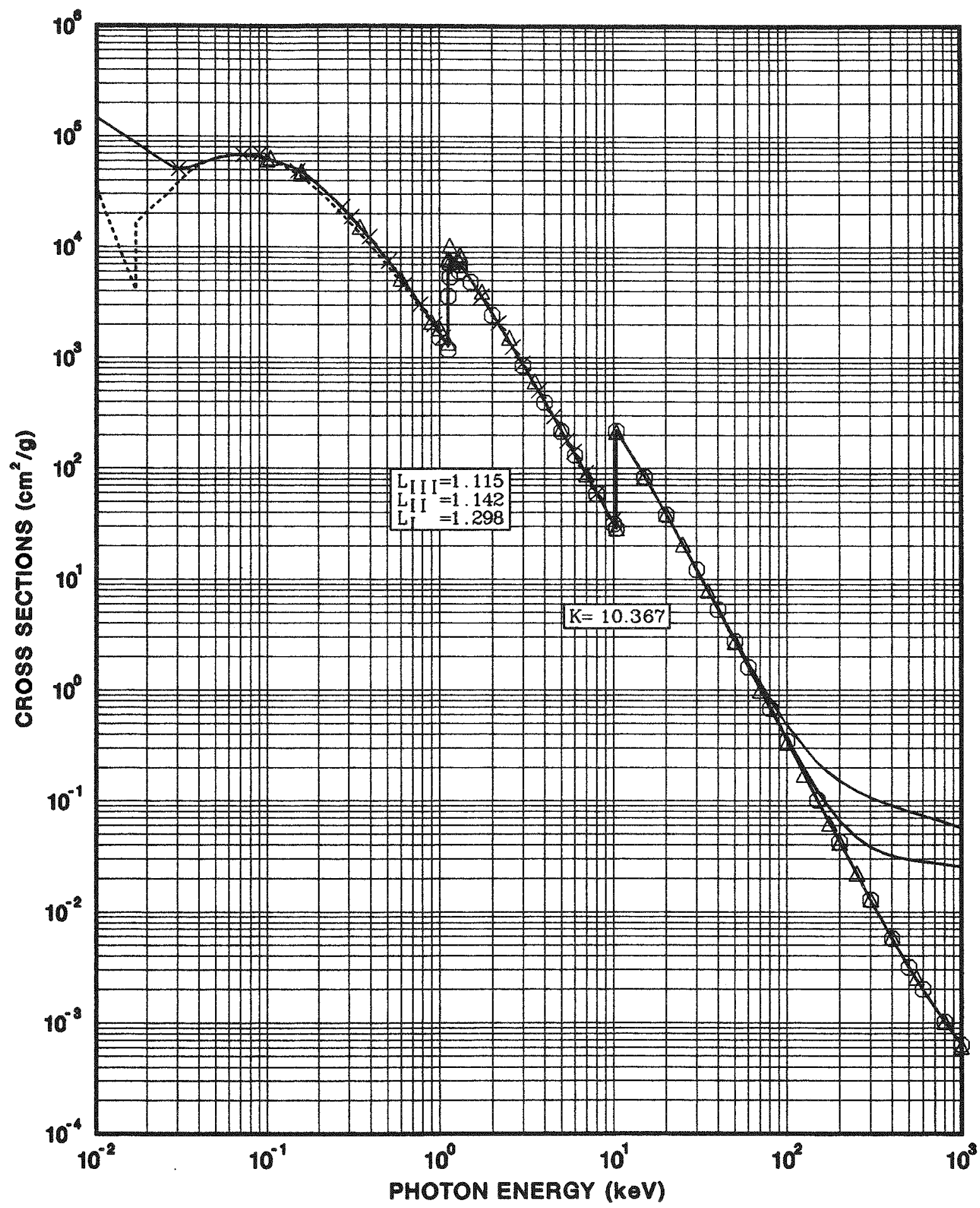

Barns/atom $=115.8 \times \mathrm{cm}^{2} / \mathrm{g}$ 


\section{GERMANIUM 32}

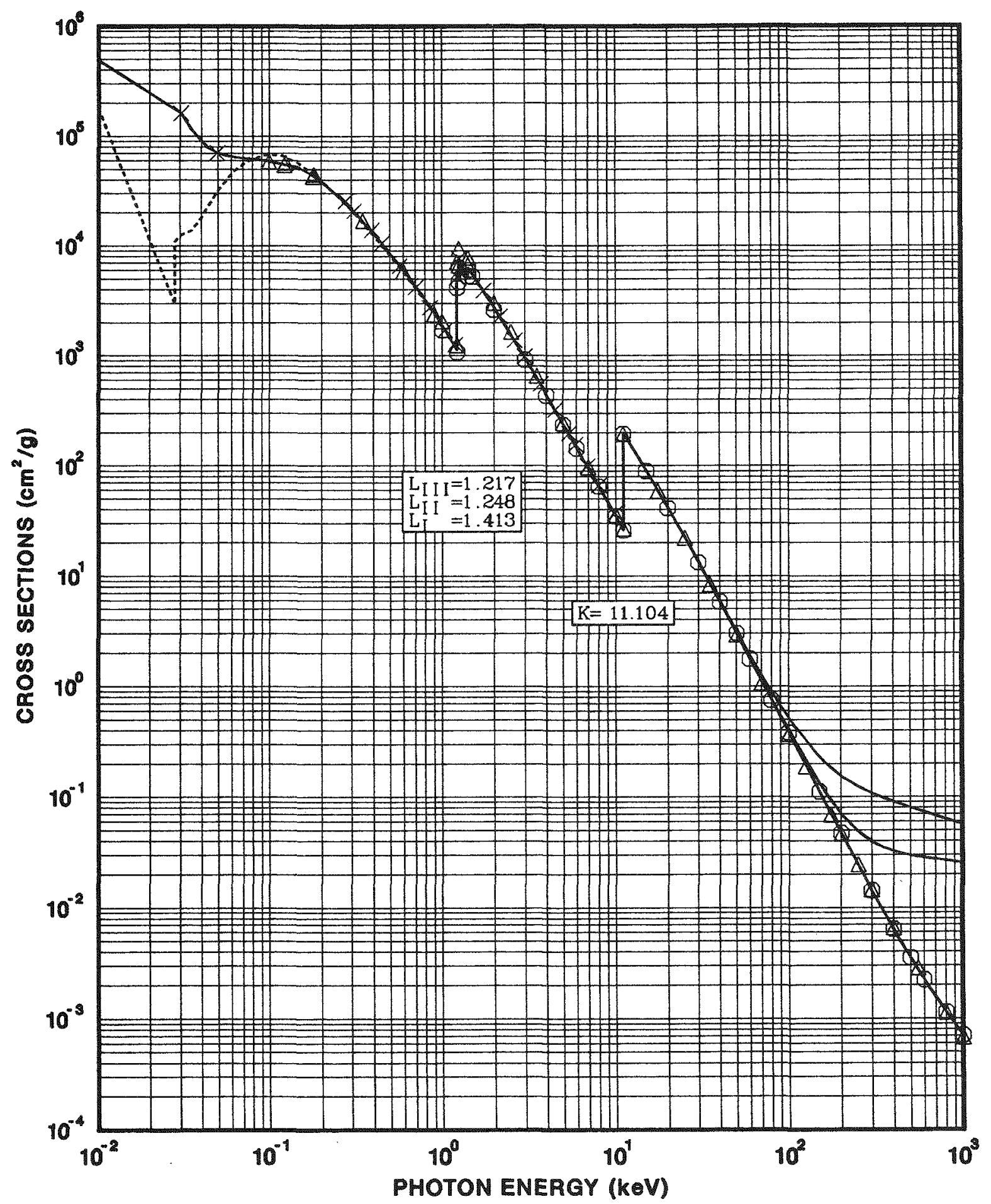

Barns/atom $=120.5 \times \mathrm{cm}^{2} / \mathrm{g}$ 


\section{ARSENIC 33}

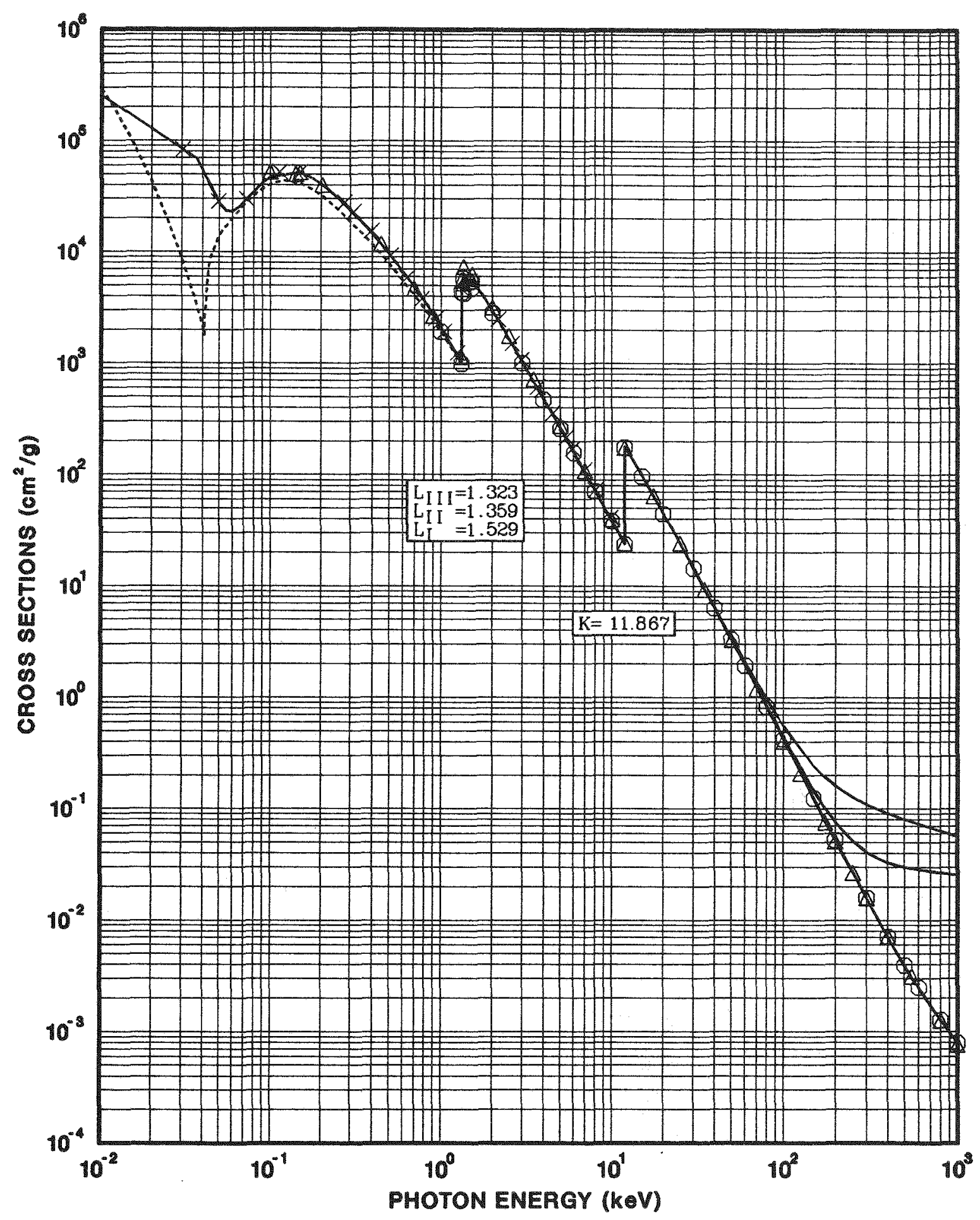

Barns/atom $=124.4 \times \mathrm{cm}^{2} / \mathrm{g}$ 
SELENIUM 34

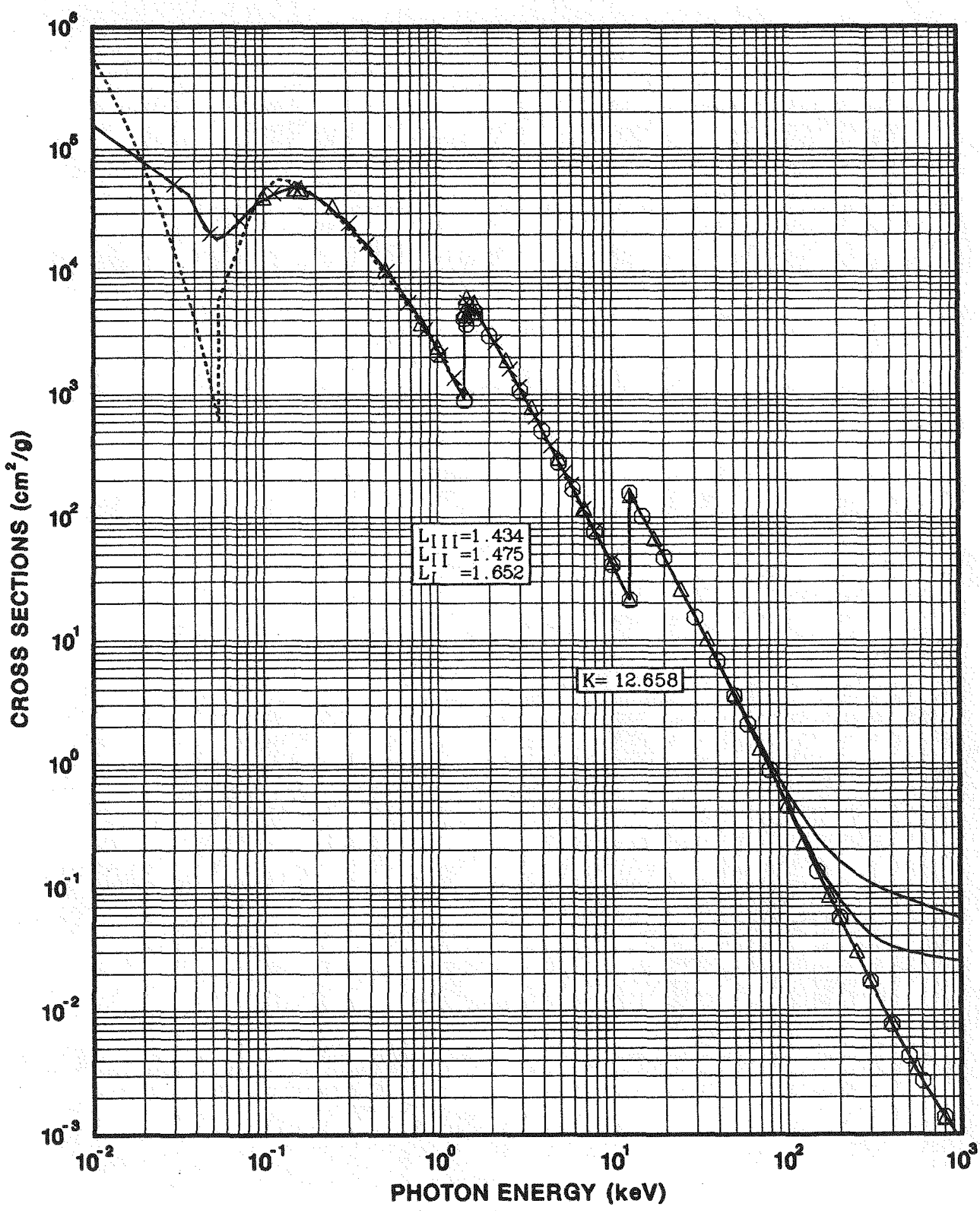

Barns $/$ atom $=131.1 \times \mathrm{cm}^{2} / \mathrm{g}$ 
BROMINE 35

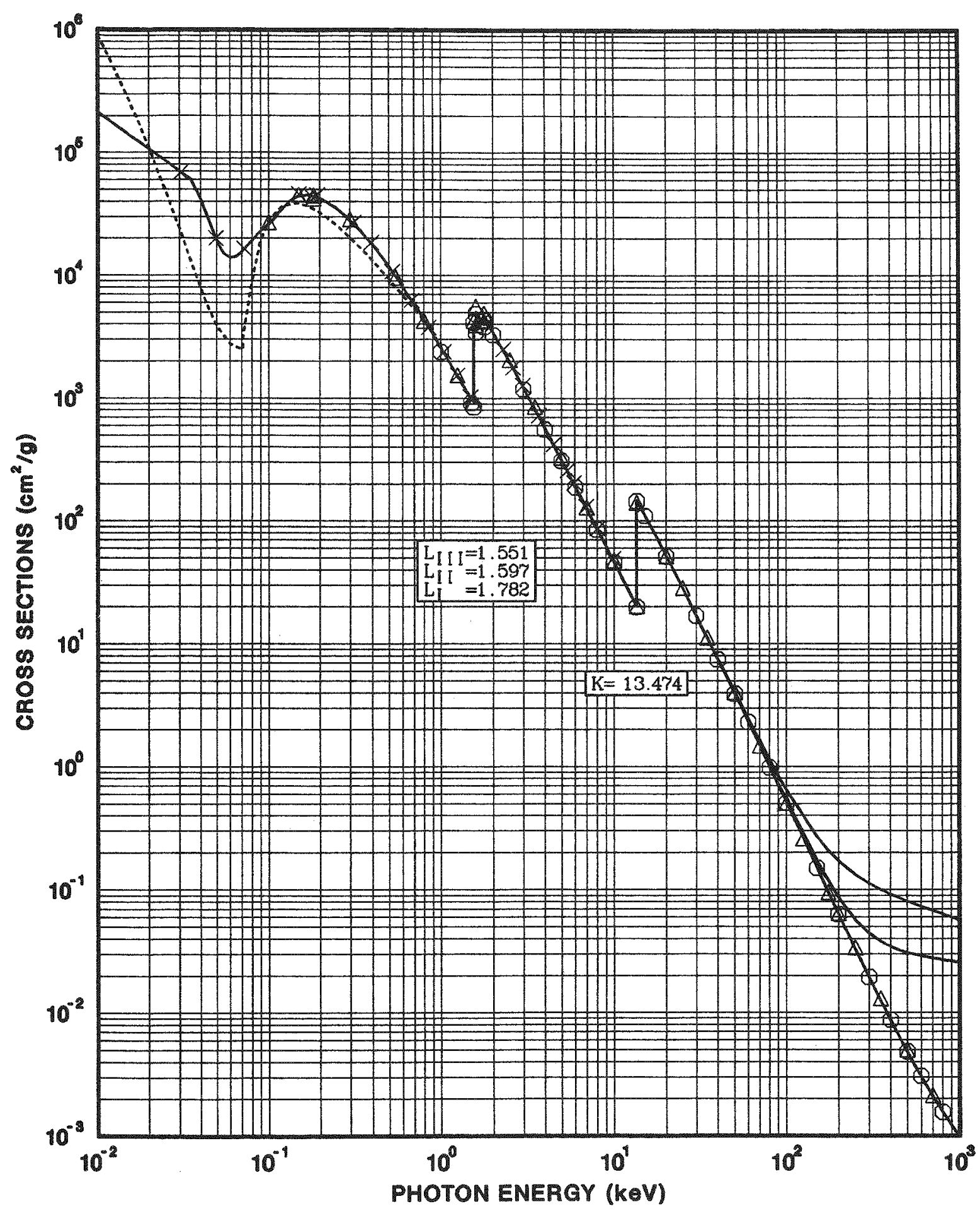

Barns/atom $=132.7 \times \mathrm{cm}^{2} / \mathrm{g}$ 
KRYPTON 36

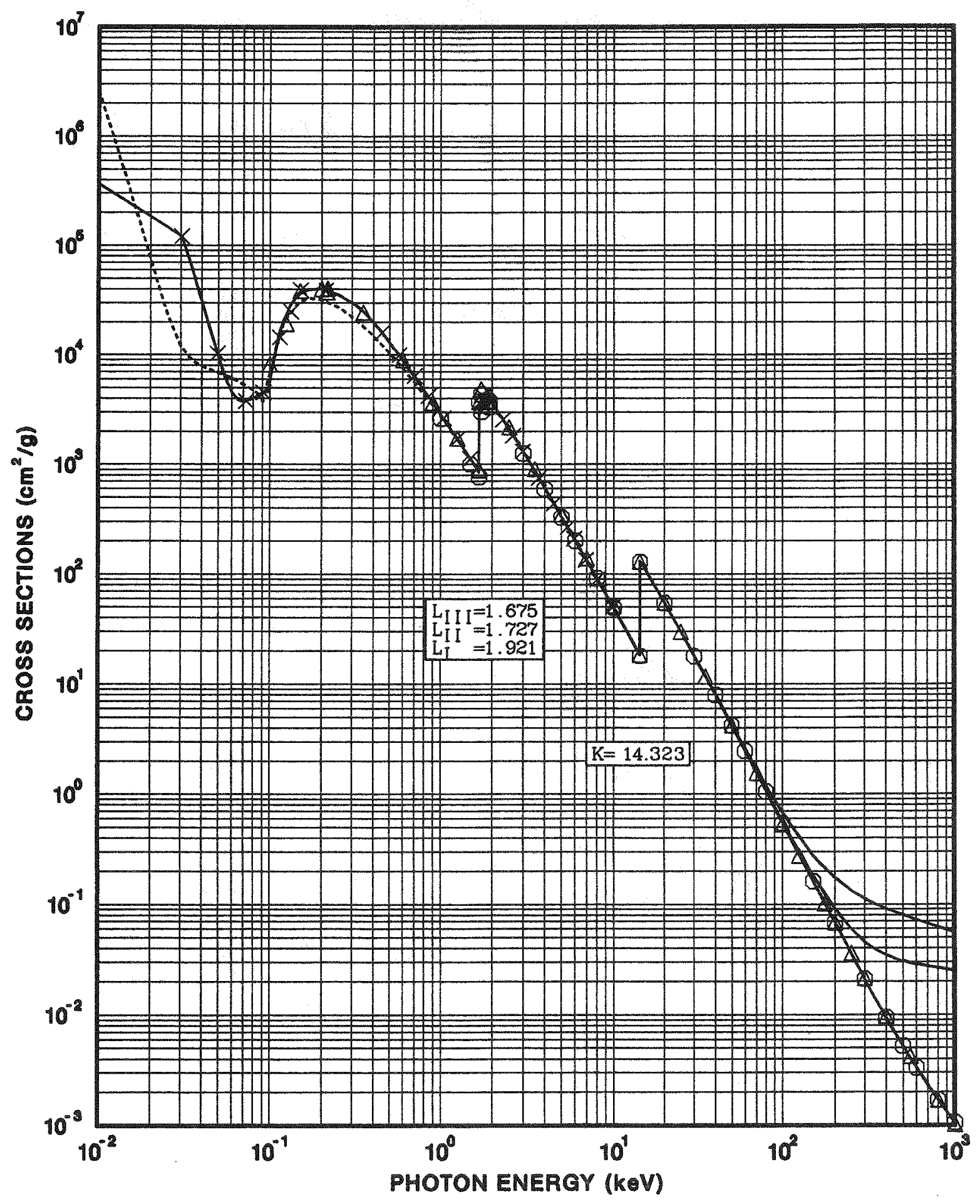

Barns/atom $=139.2 \times \mathrm{cm}^{2} / \mathrm{g}$ 


\section{RUBIDIUM 37}

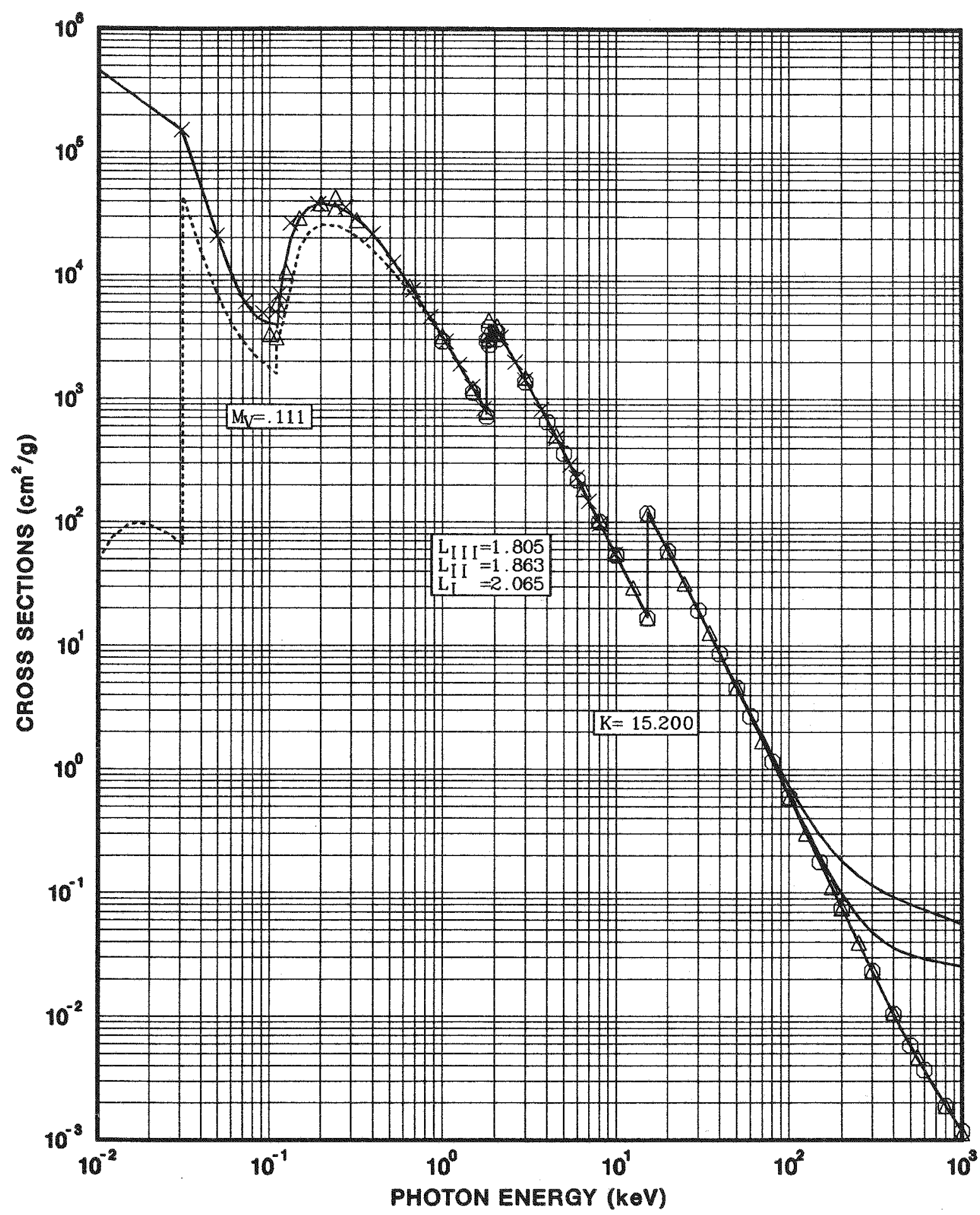

Barns/atom $=141.9 \times \mathrm{cm}^{2} / \mathrm{g}$ 


\section{STRONTIUM 38}

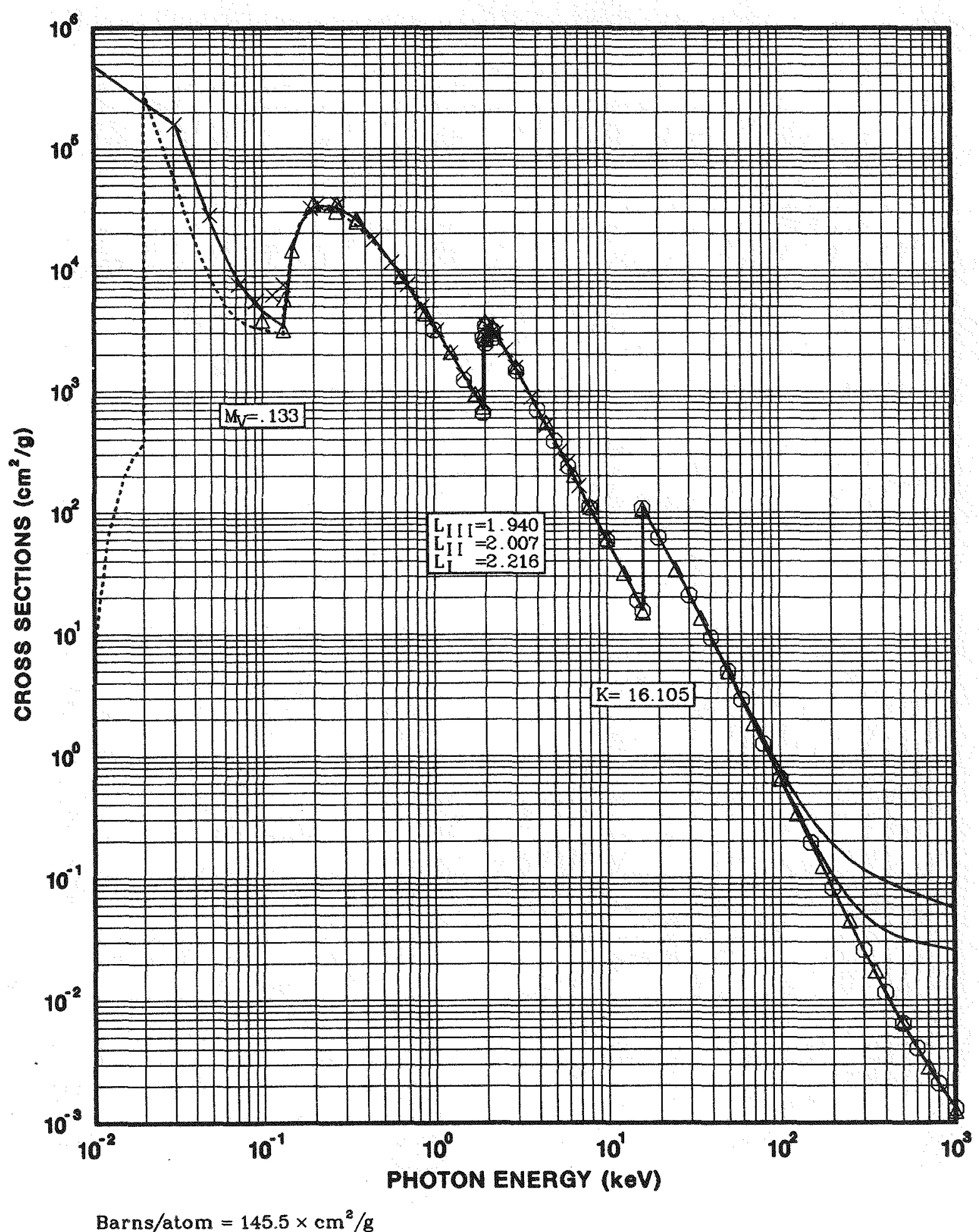


YTTRIUM 39

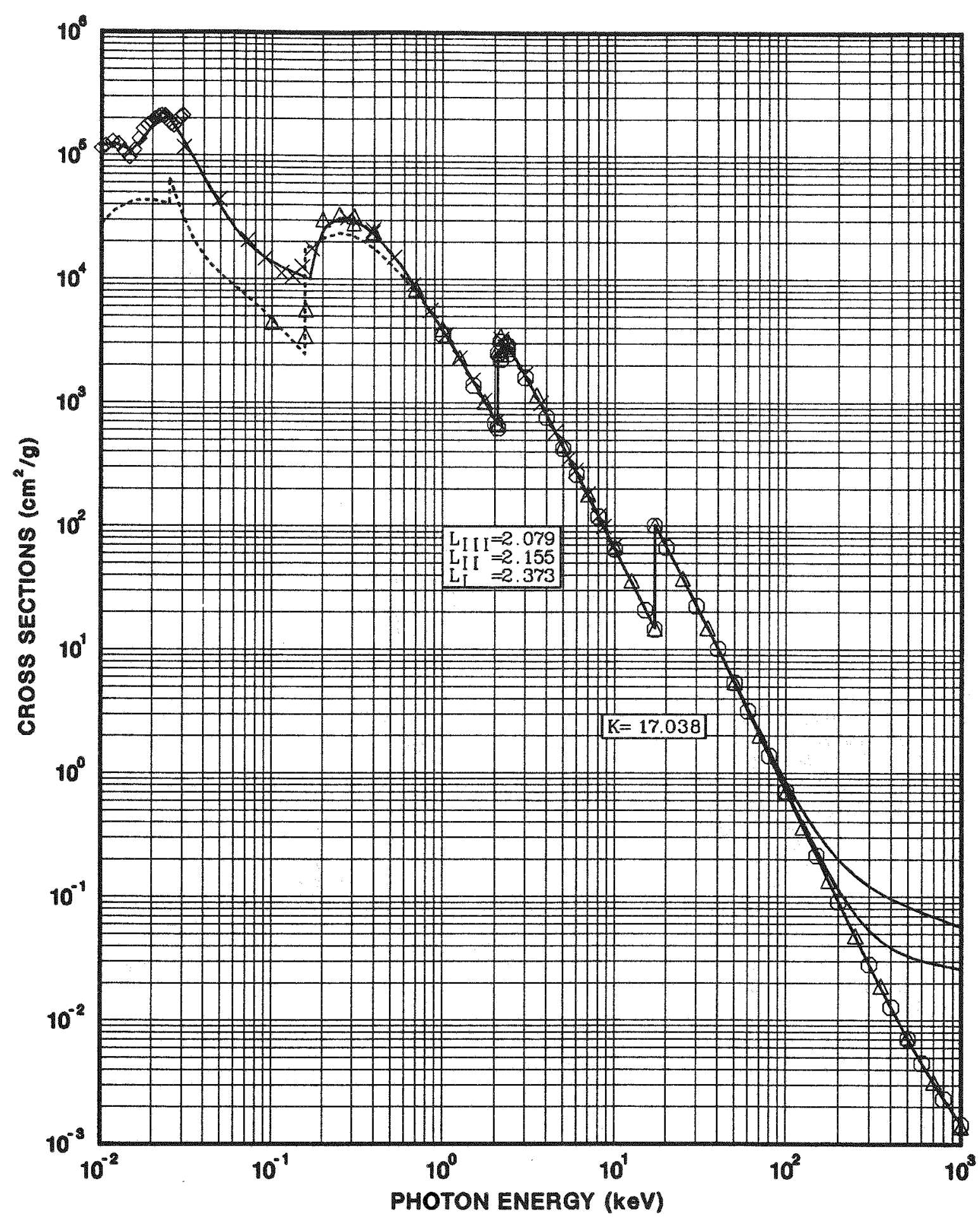

Barns $/$ atom $=147.6 \times \mathrm{cm}^{2} / \mathrm{g}$ 


\section{ZIRCONIUM 40}

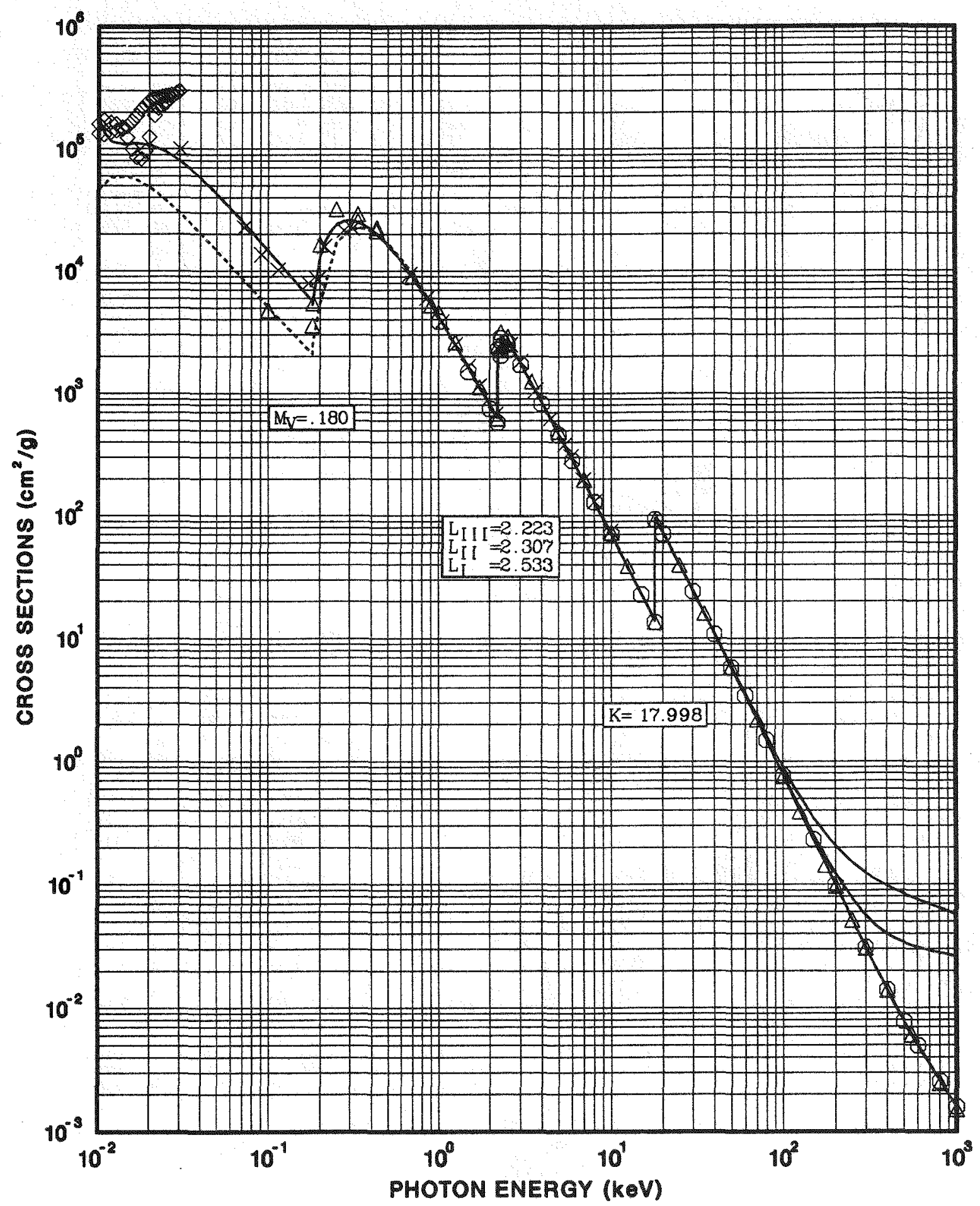

Barns/atom $=151.5 \times \mathrm{cm}^{2} / \mathrm{g}$ 


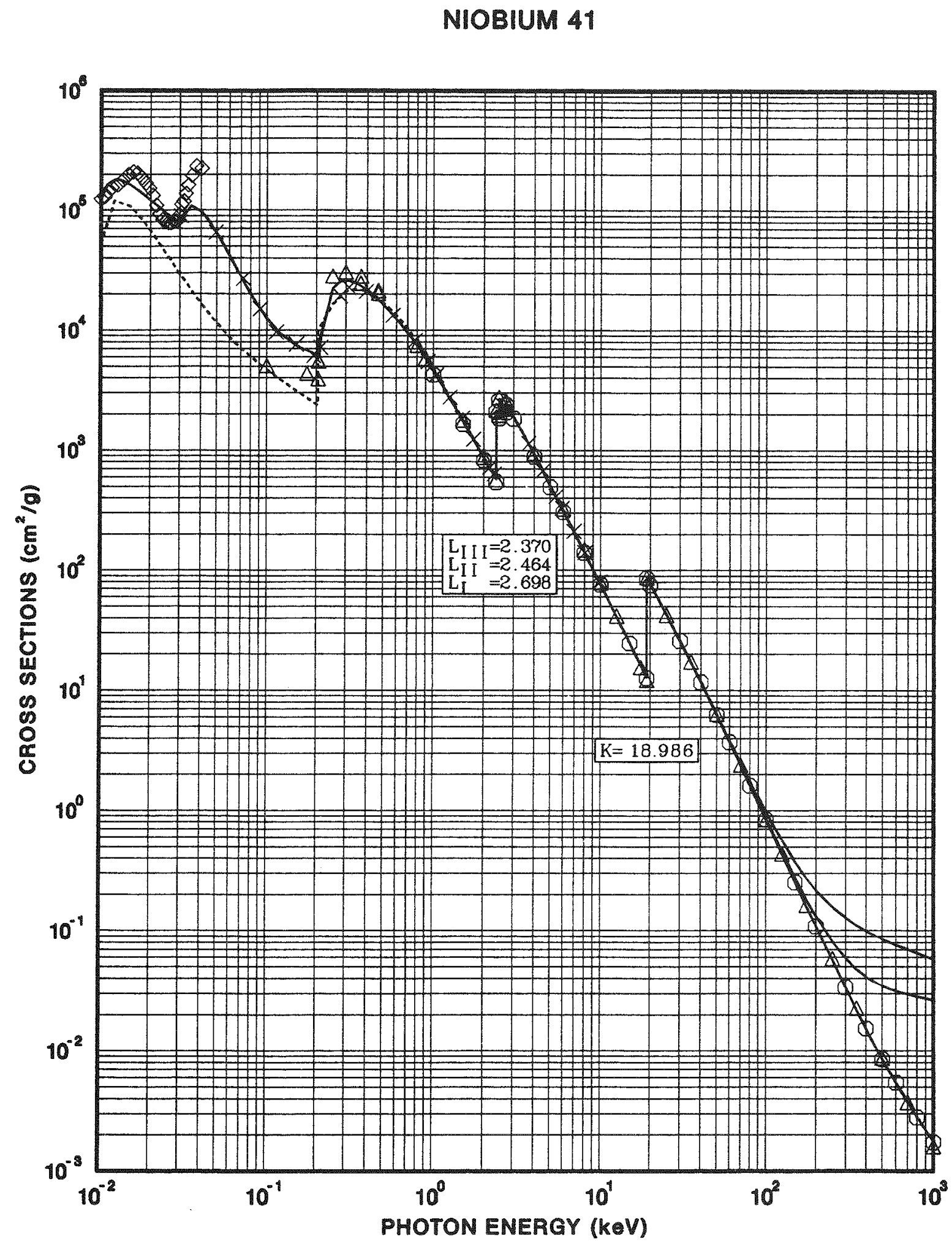

Barns $/$ atom $=154.3 \times \mathrm{cm}^{2} / \mathrm{g}$ 


\section{MOLYBDENUM 42}

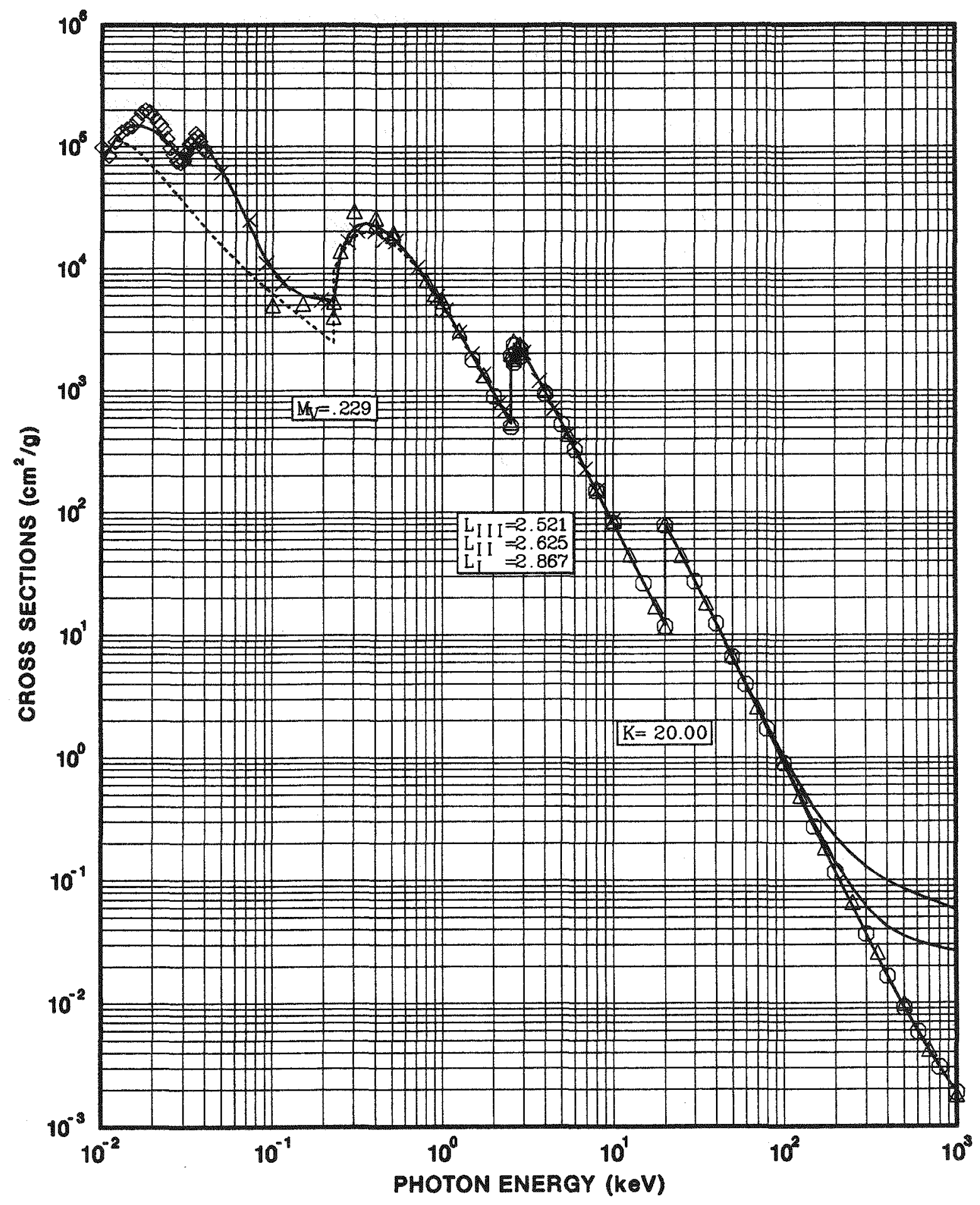

Barns/atom $=159.3 \times \mathrm{cm}^{2} / \mathrm{g}$ 
TECHNETIUM 43

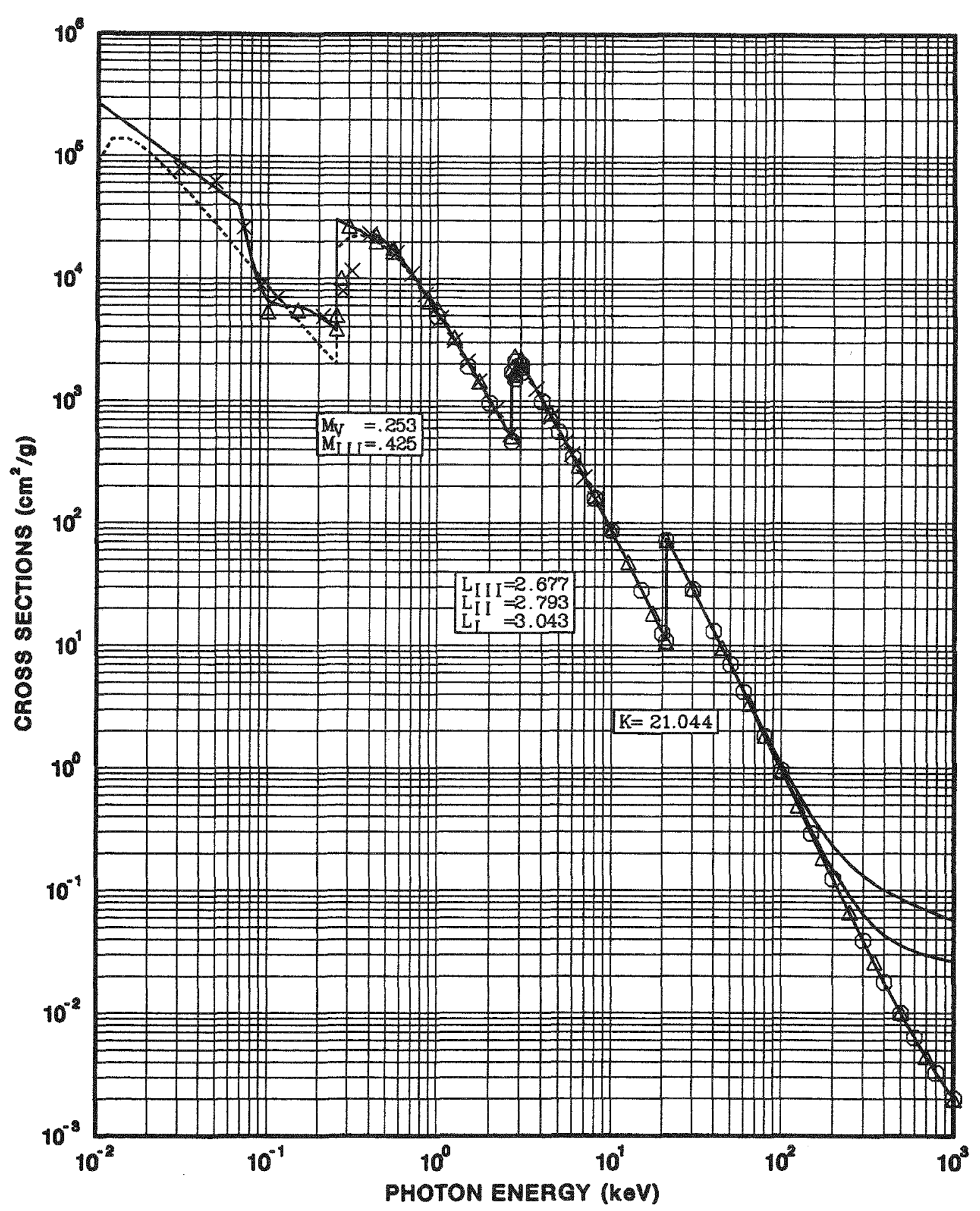

Barns/atom $=162.7 \times \mathrm{cm}^{2} / \mathrm{g}$ 


\section{RUTHENIUM 44}

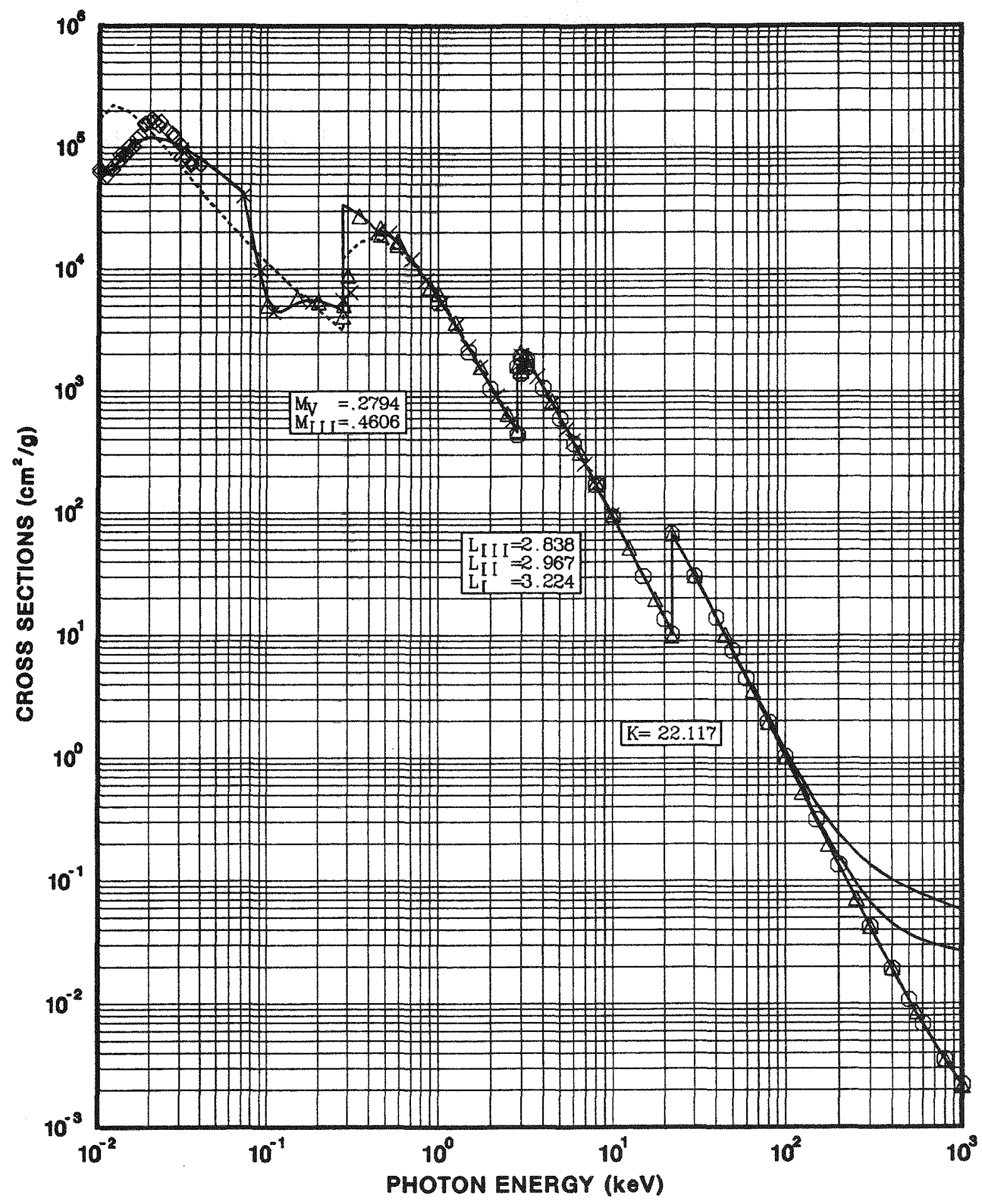

Barns $/$ atom $=167.8 \times \mathrm{cm}^{2} / \mathrm{g}$ 


\section{RHODIUM 45}

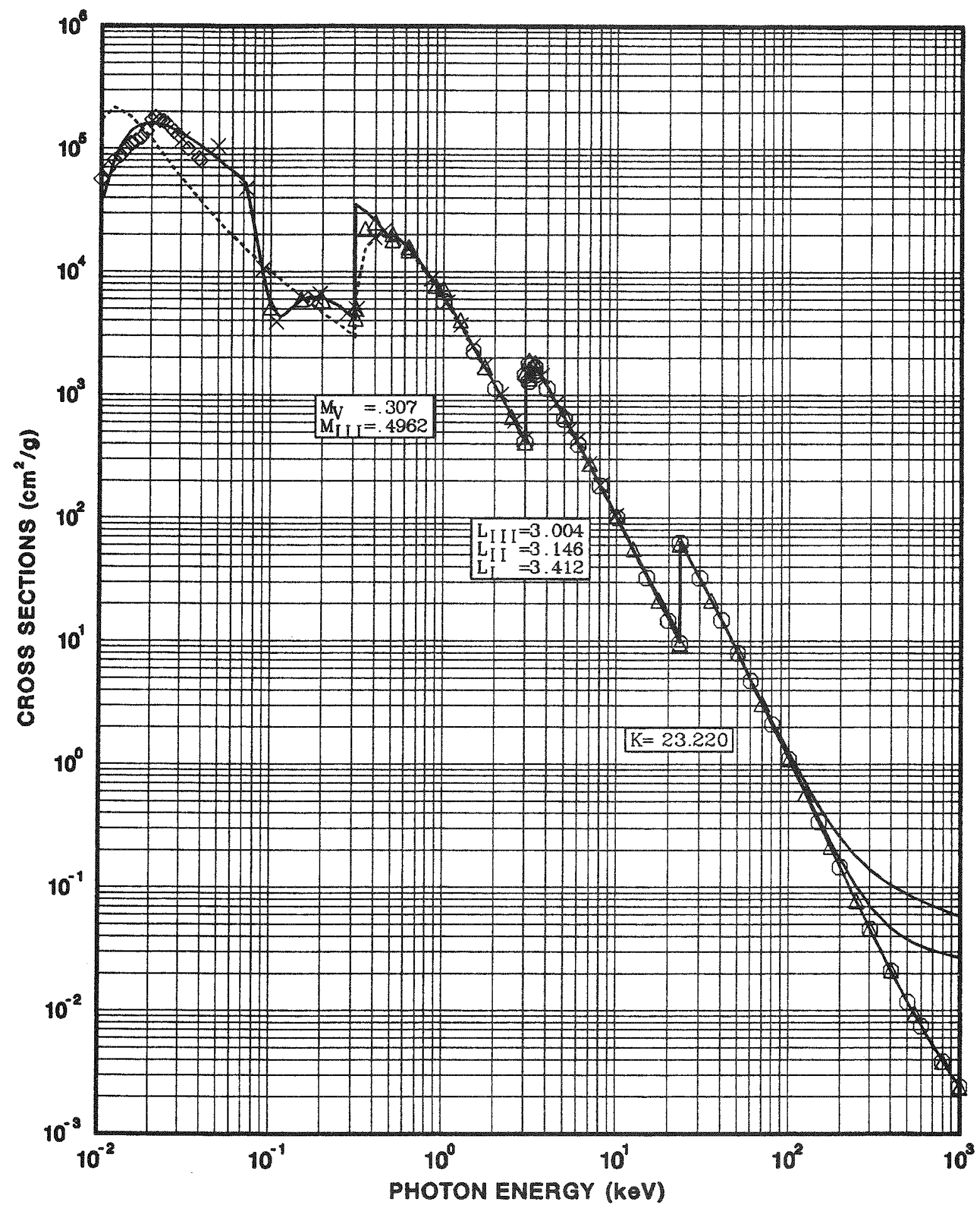

Barns/atom $=170.9 \times \mathrm{cm}^{2} / \mathrm{g}$ 
PALLADIUM 46

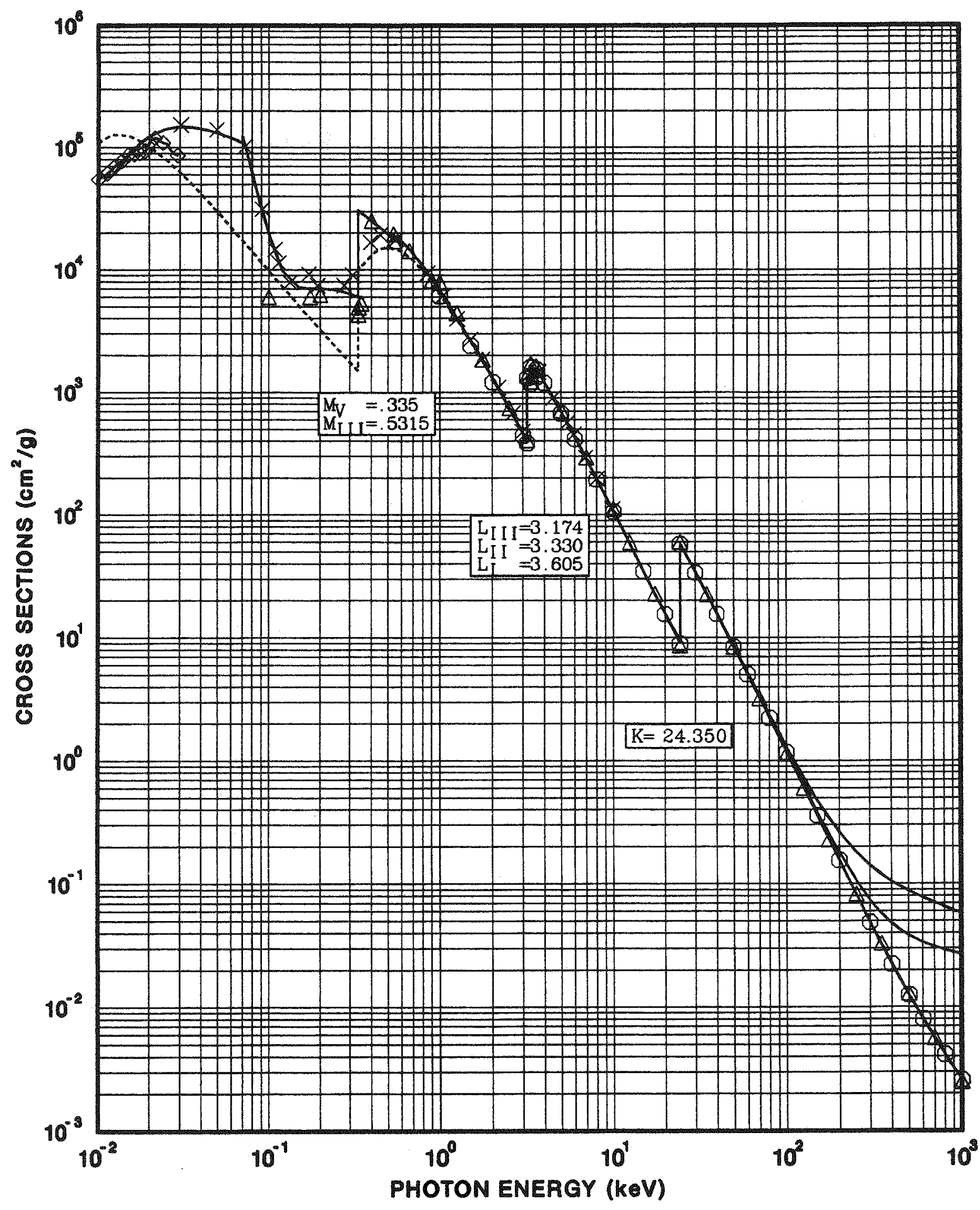

Barns/atom $=176.7 \times \mathrm{cm}^{2} / \mathrm{g}$ 
SILVER 47

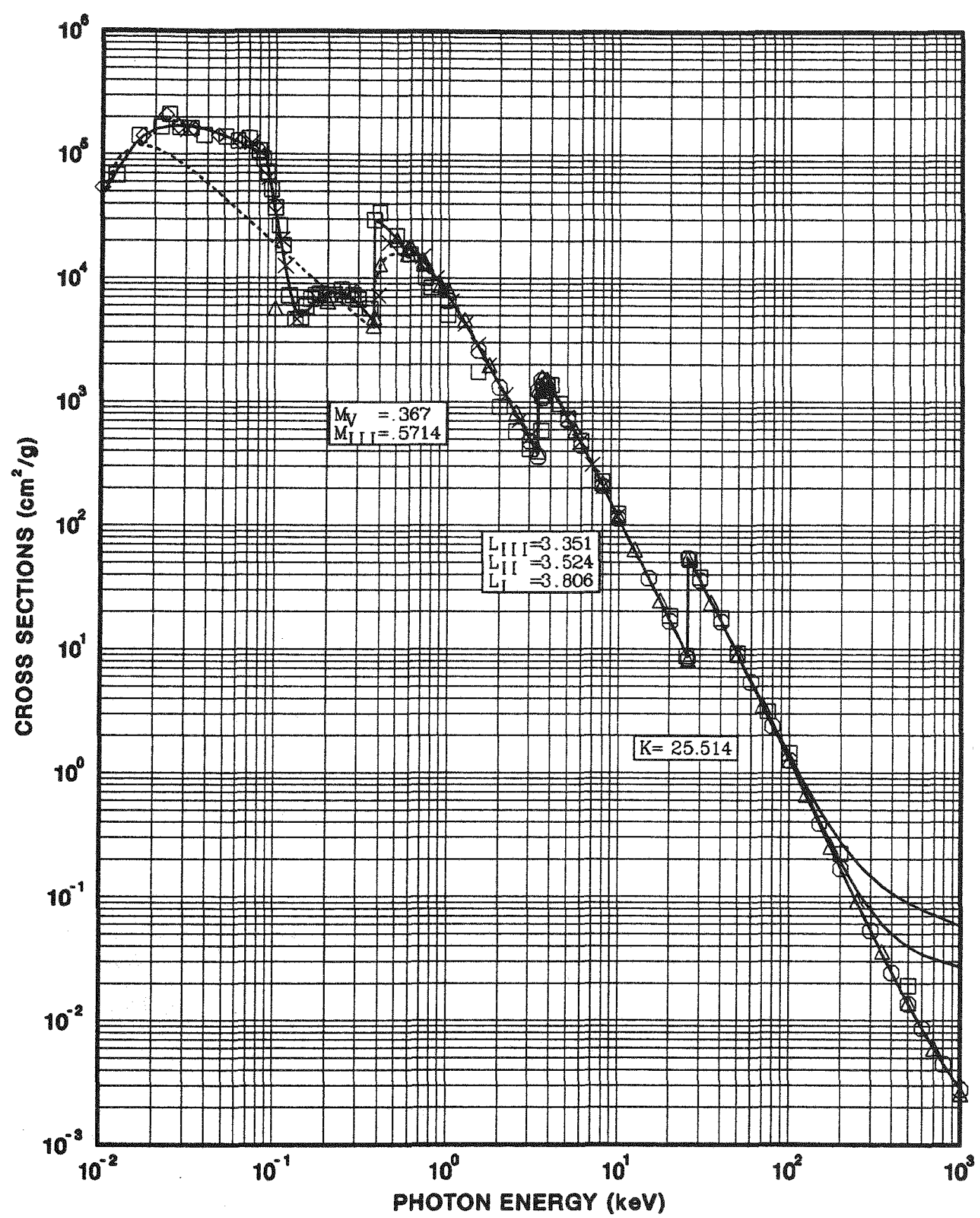

Barns/atom $=179.1 \times \mathrm{cm}^{2} / \mathrm{g}$ 
CADMIUM 48

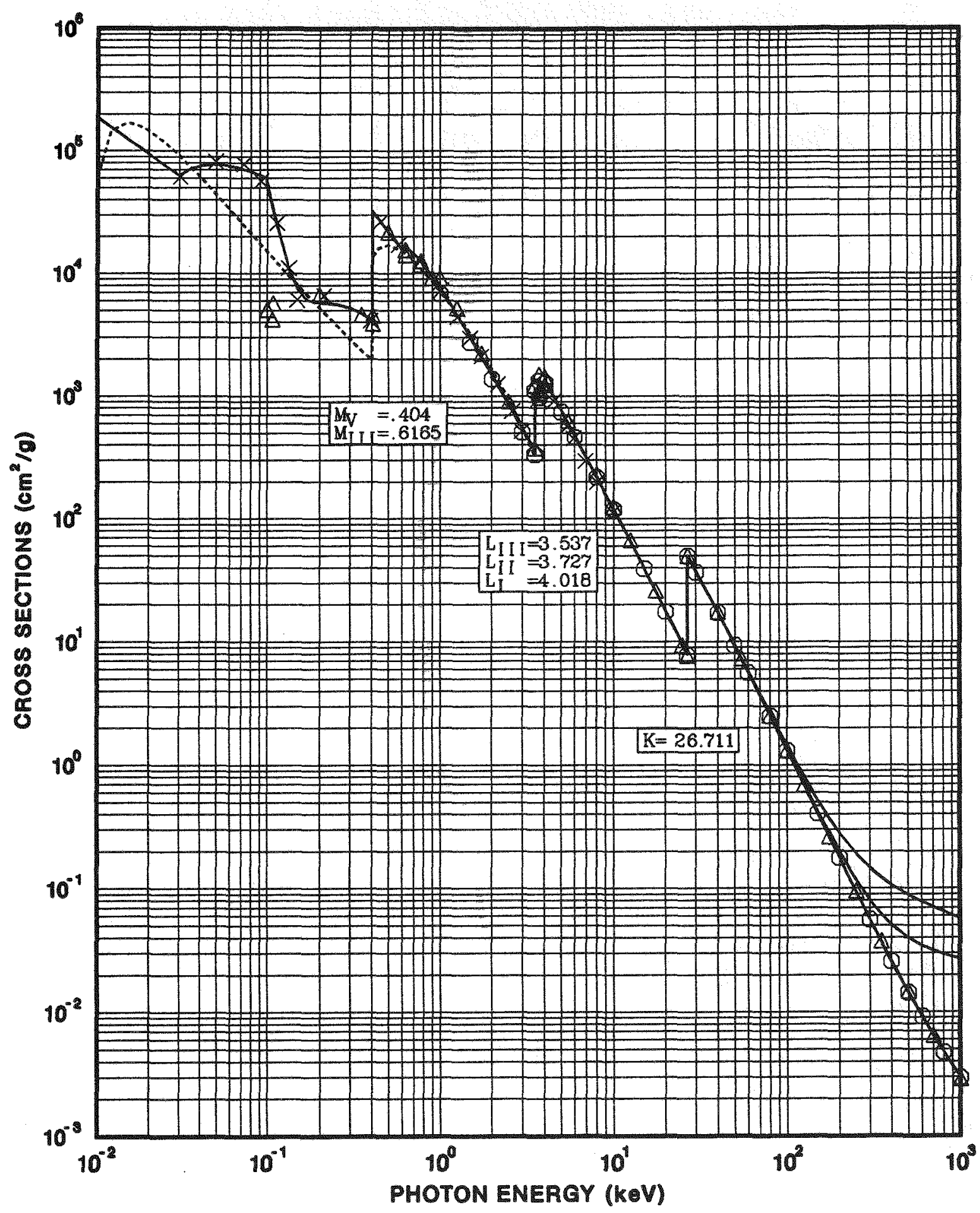

Barns $/$ atom $=186.7 \times \mathrm{cm}^{2} / \mathrm{g}$ 


\section{INDIUM 49}

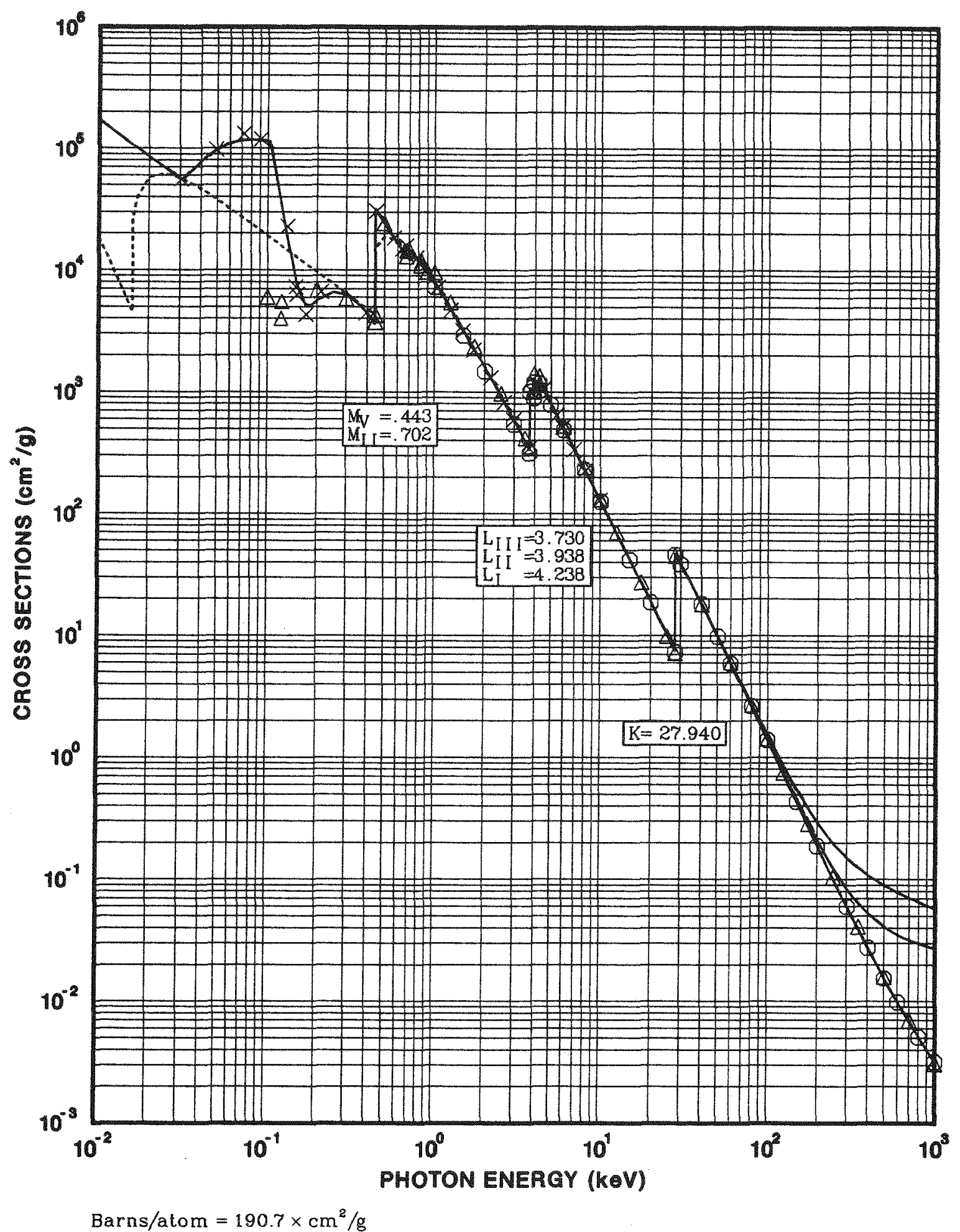




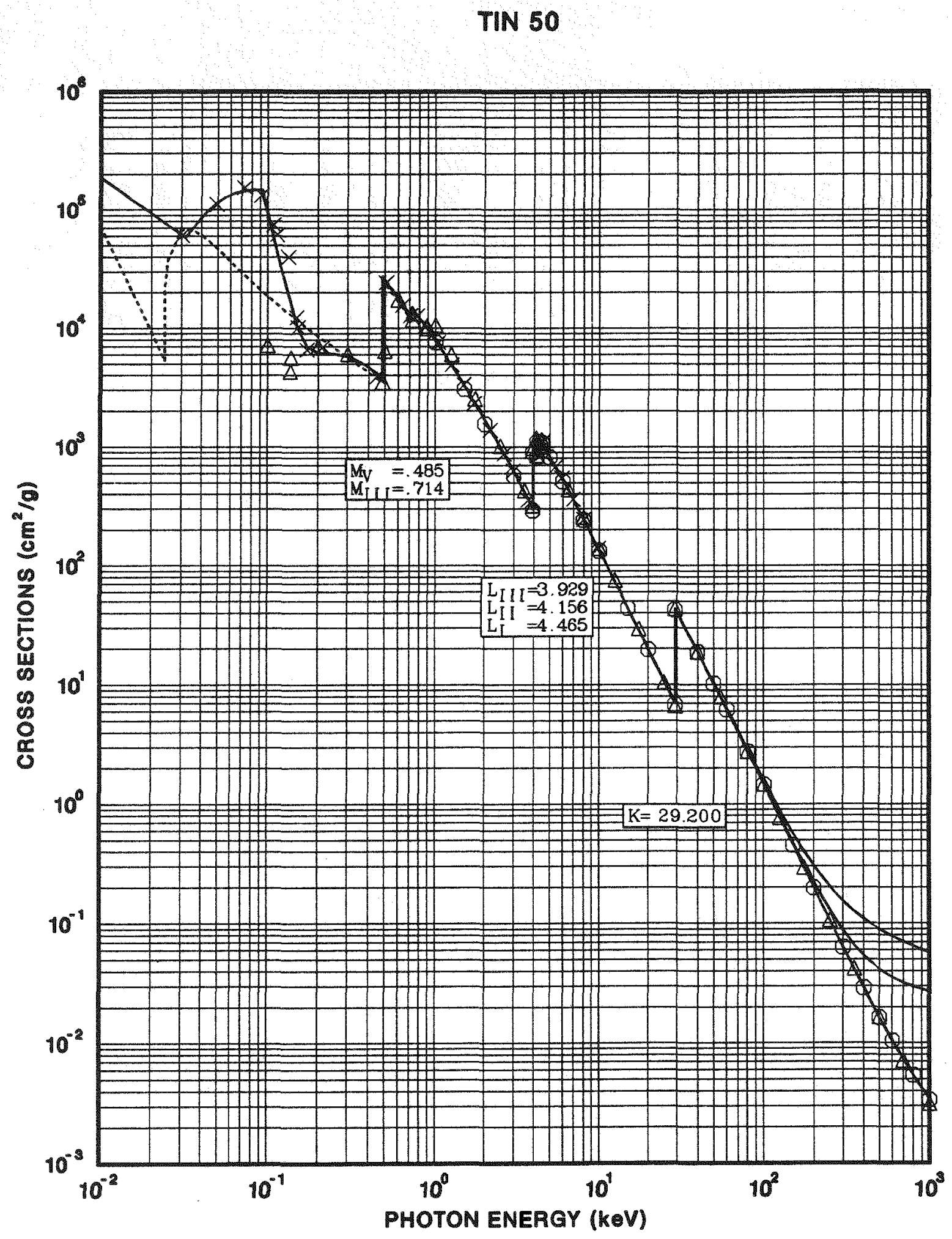

Barns/atom $=197.1 \times \mathrm{cm}^{2} / \mathrm{g}$ 


\section{ANTIMONY 51}

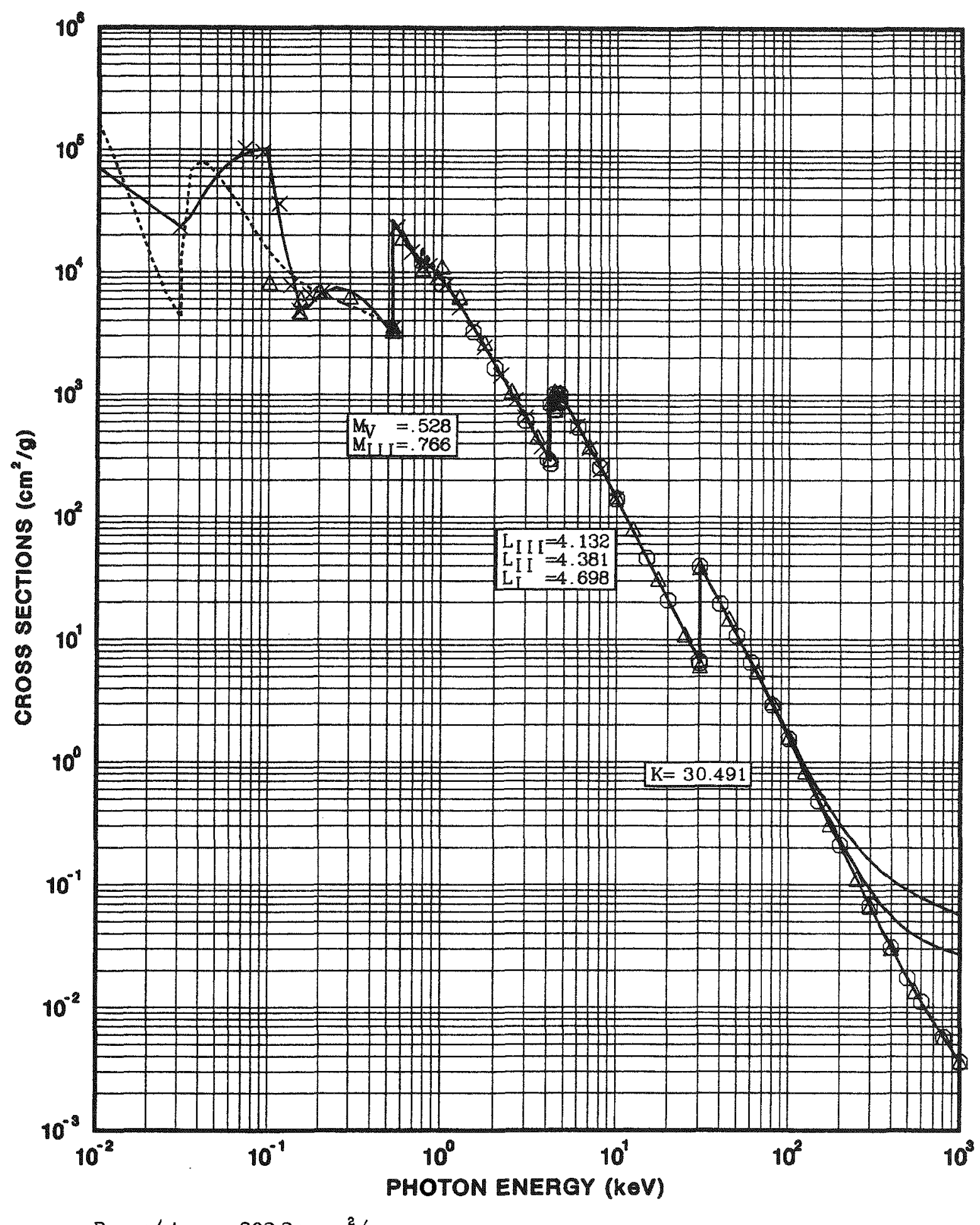

Barns/atom $=202.2 \times \mathrm{cm}^{2} / \mathrm{g}$ 
TELLURIUM 52

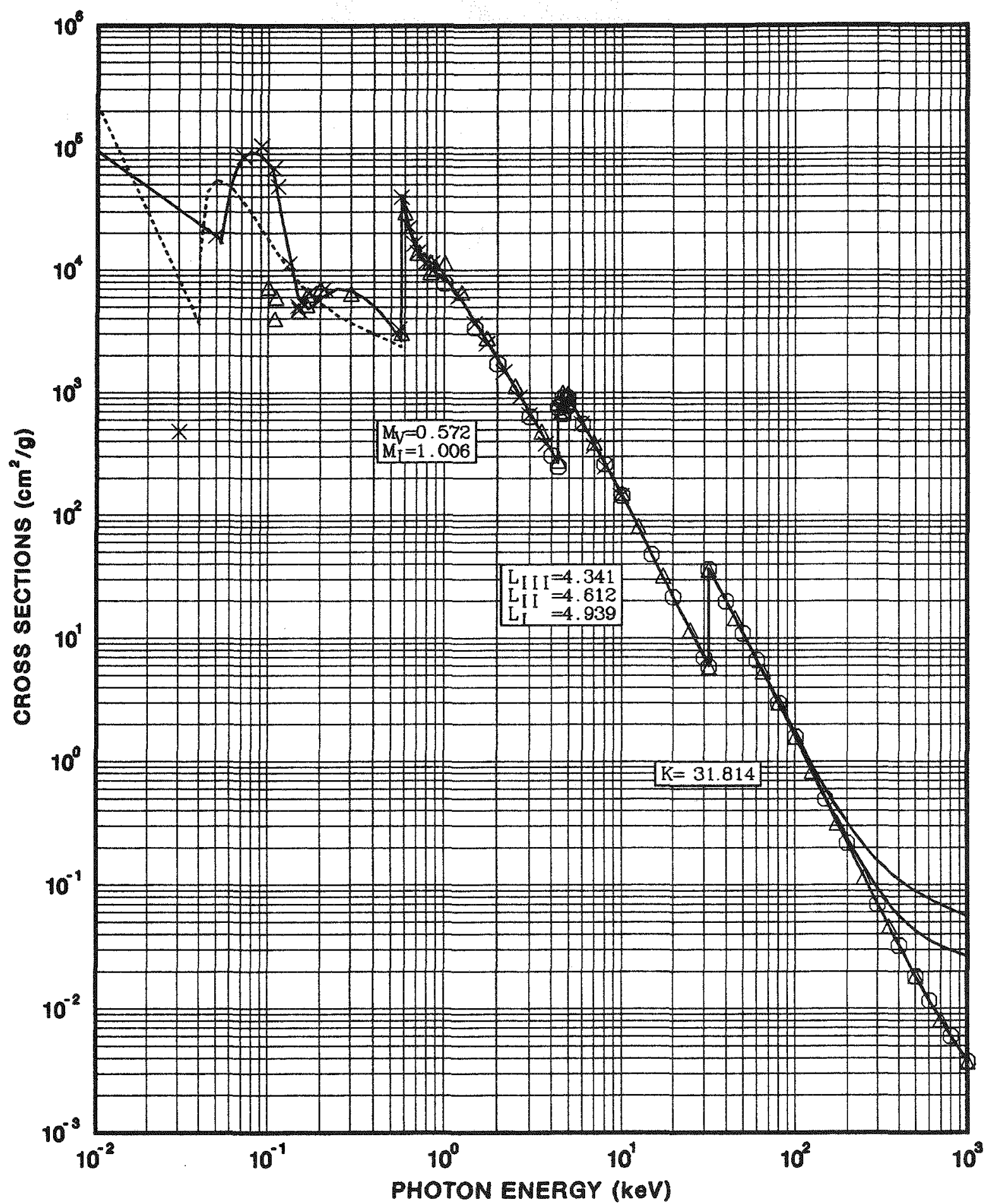

Barns/atom $=211.9 \times \mathrm{cm}^{2} / \mathrm{g}$ 
IODINE 53

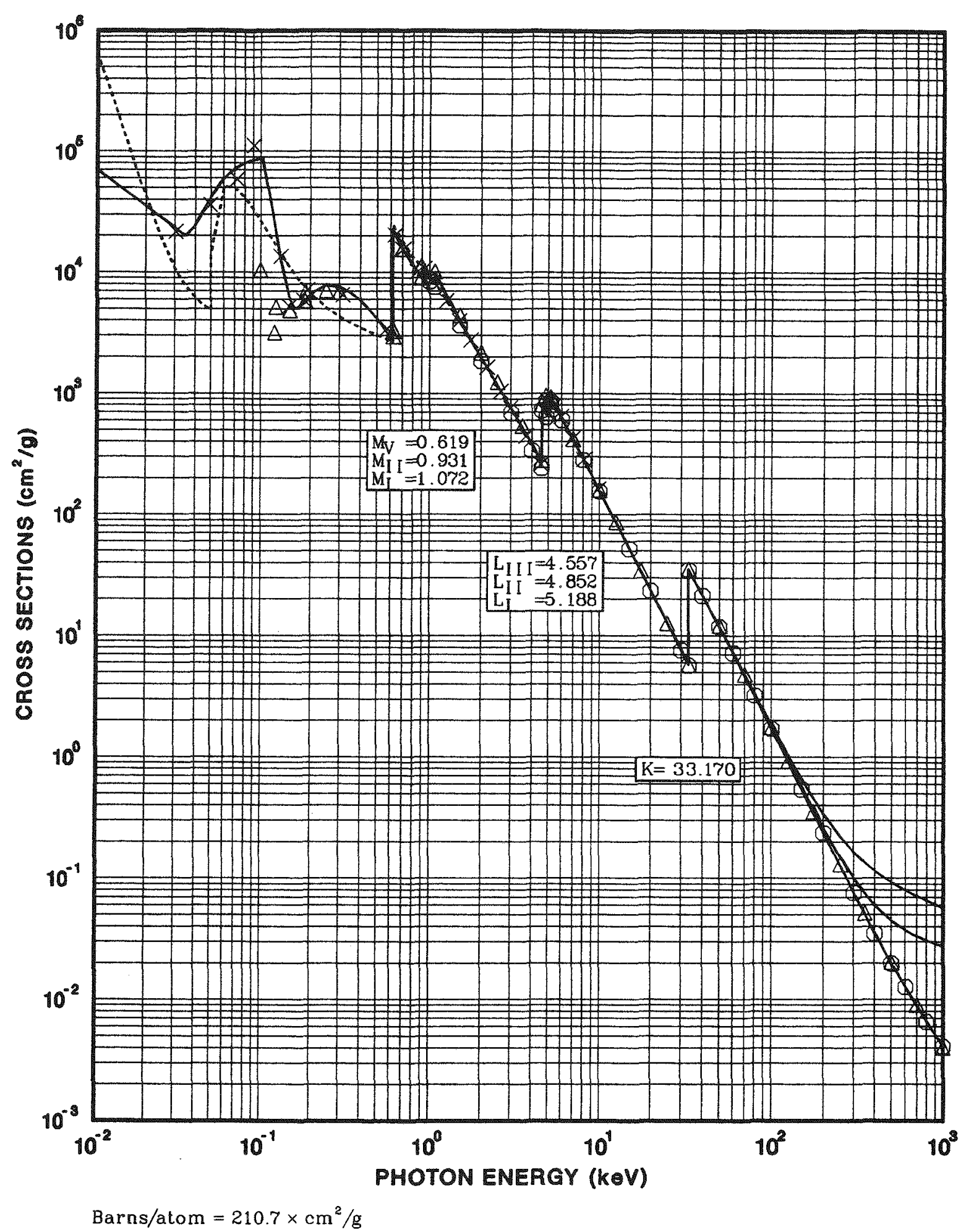




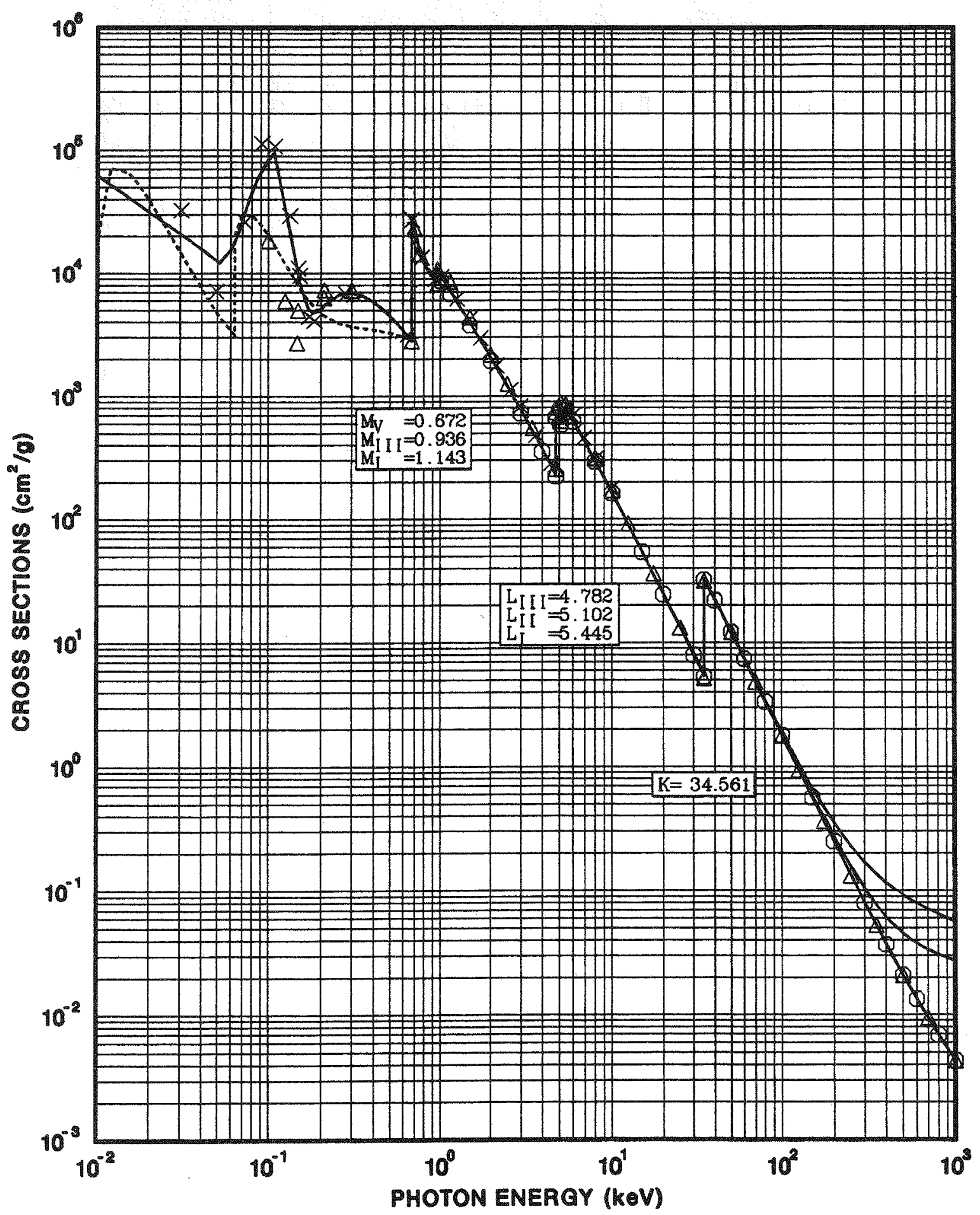

Barns/atom $=218.0 \times \mathrm{cm}^{2} / \mathrm{g}$ 


\section{CESIUM 55}

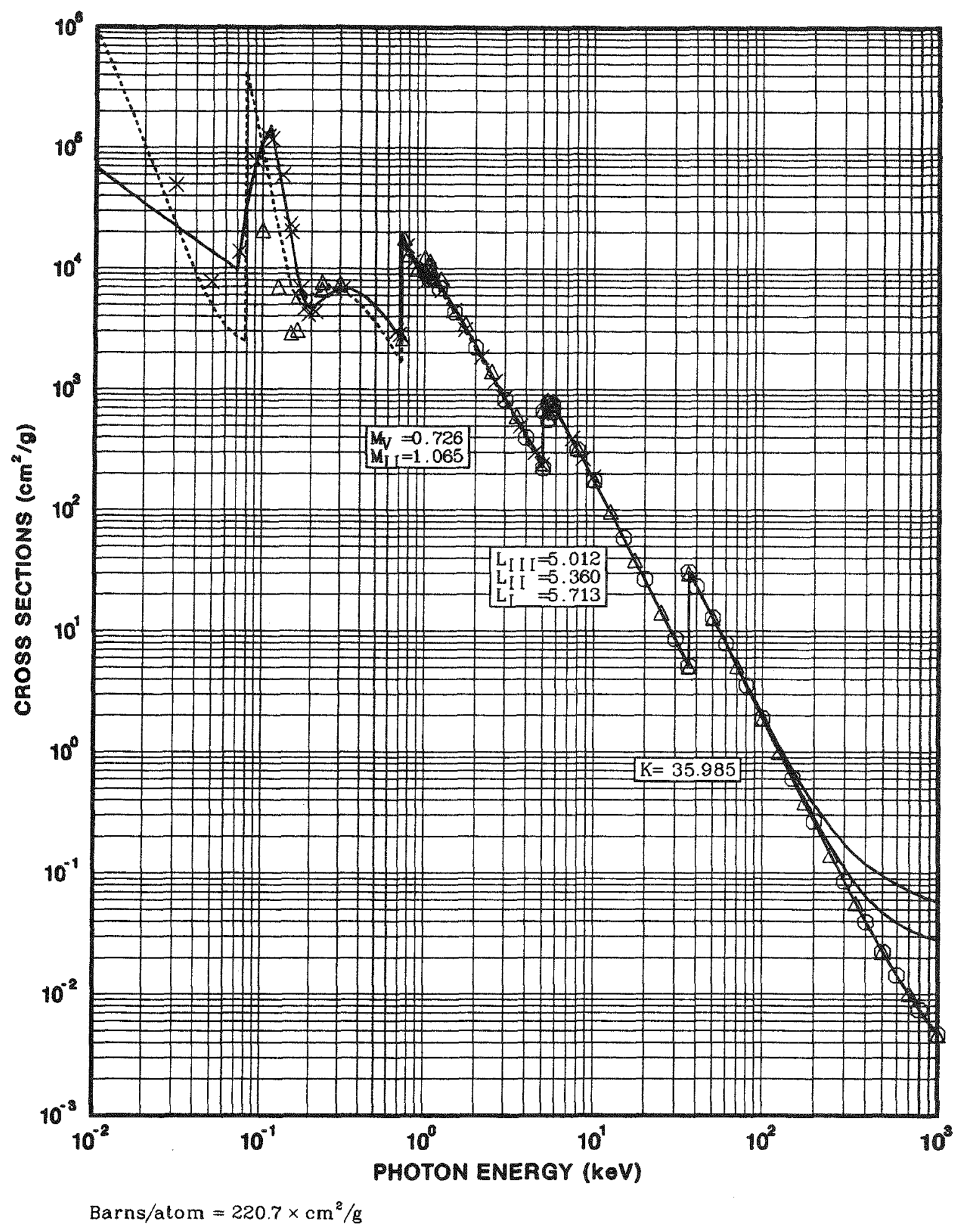


BARIUM 56

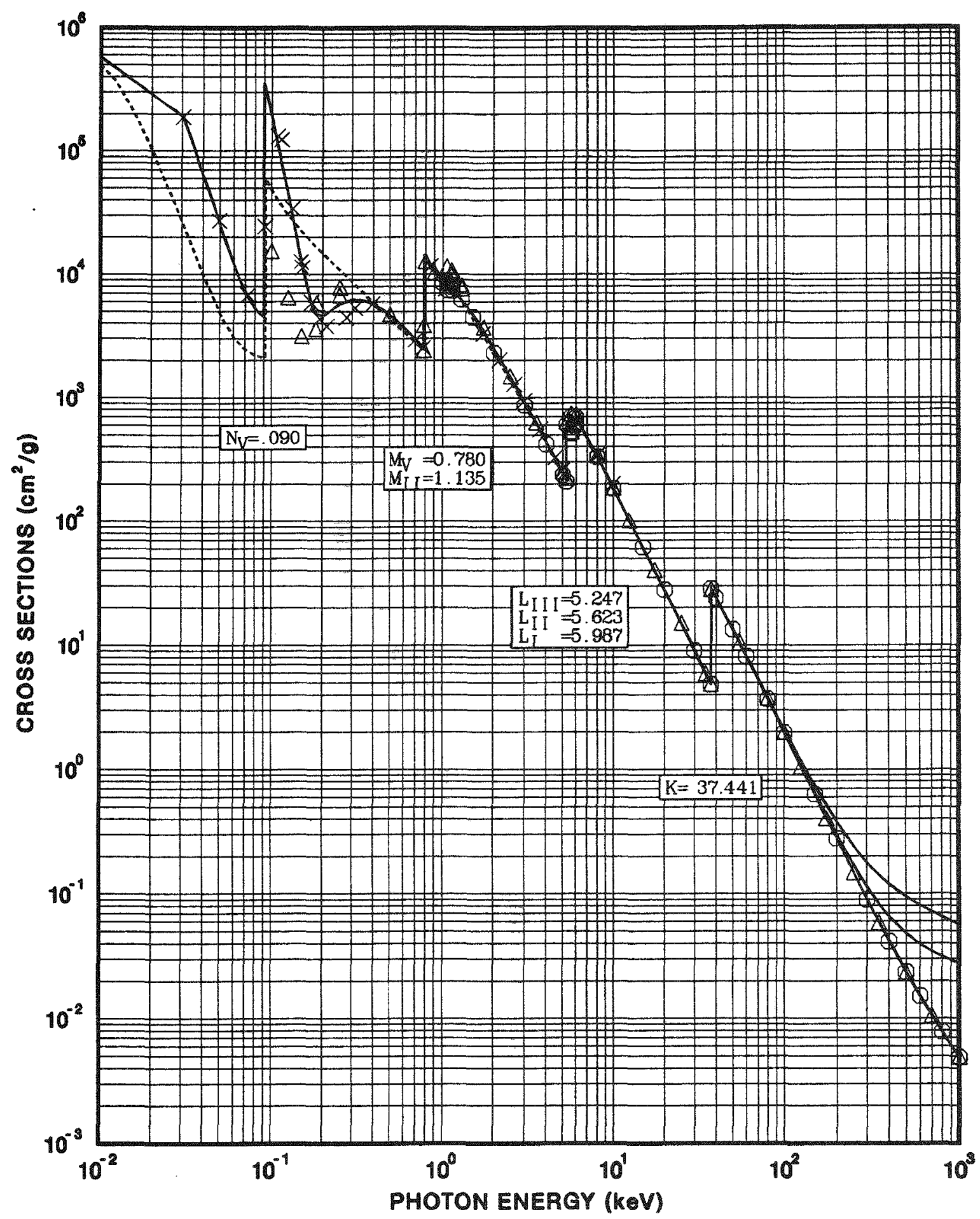

Barns/atom $=228.0 \times \mathrm{cm}^{2} / \mathrm{g}$ 


\section{LANTHANUM 57}

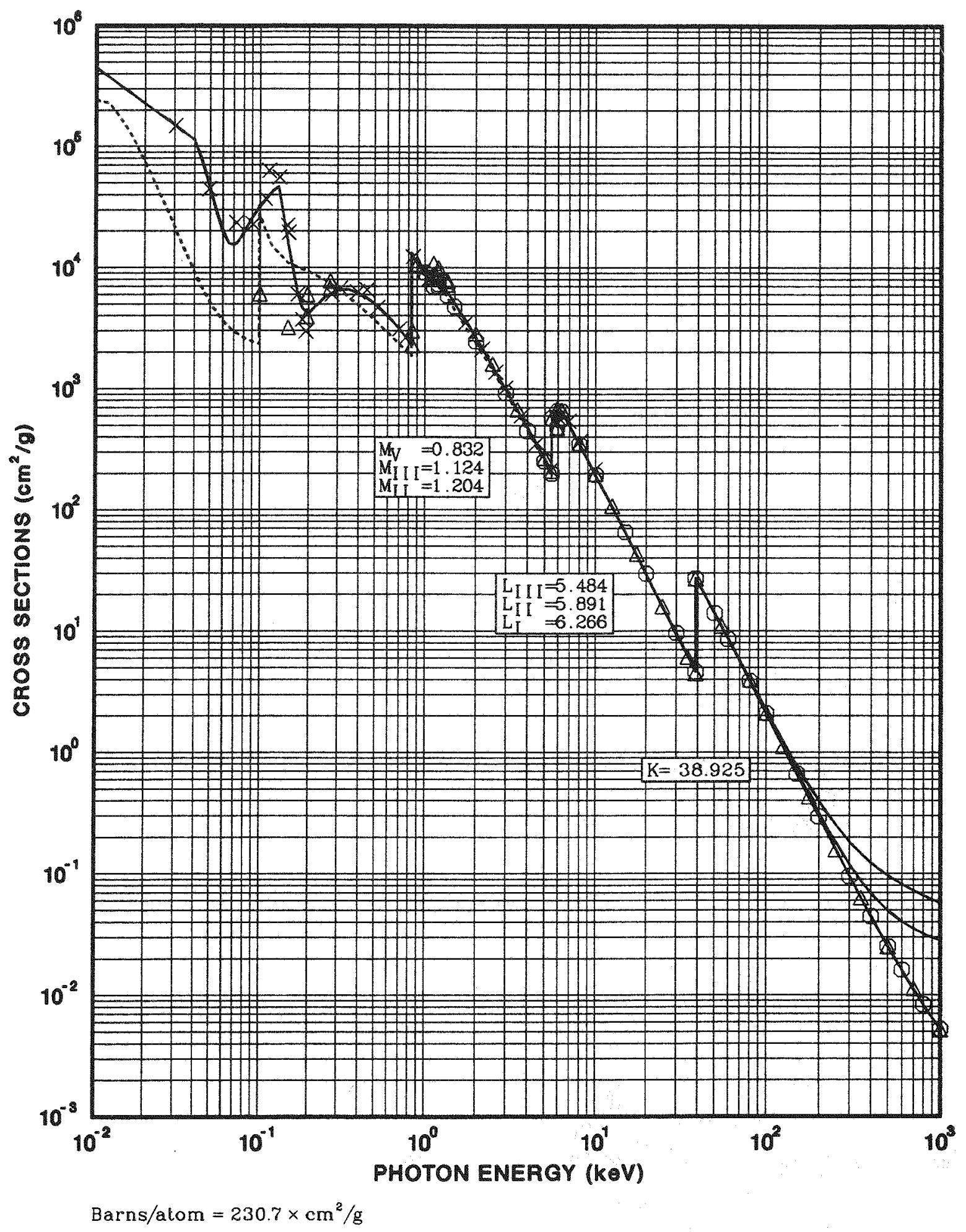


CERIUM 58

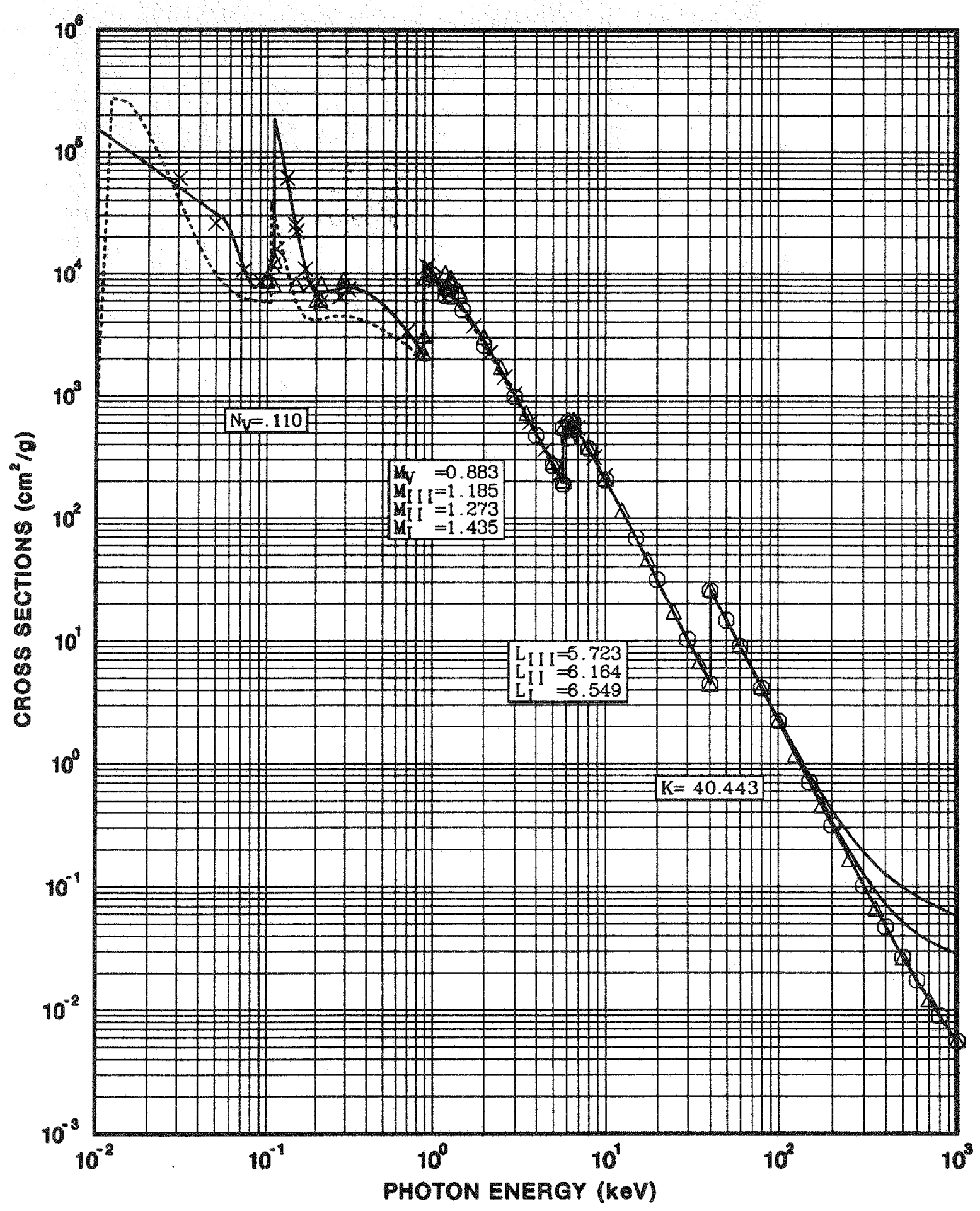

Barns/atom $=232.7 \times \mathrm{cm}^{2} / \mathrm{g}$ 
PRASEODYMIUM 59

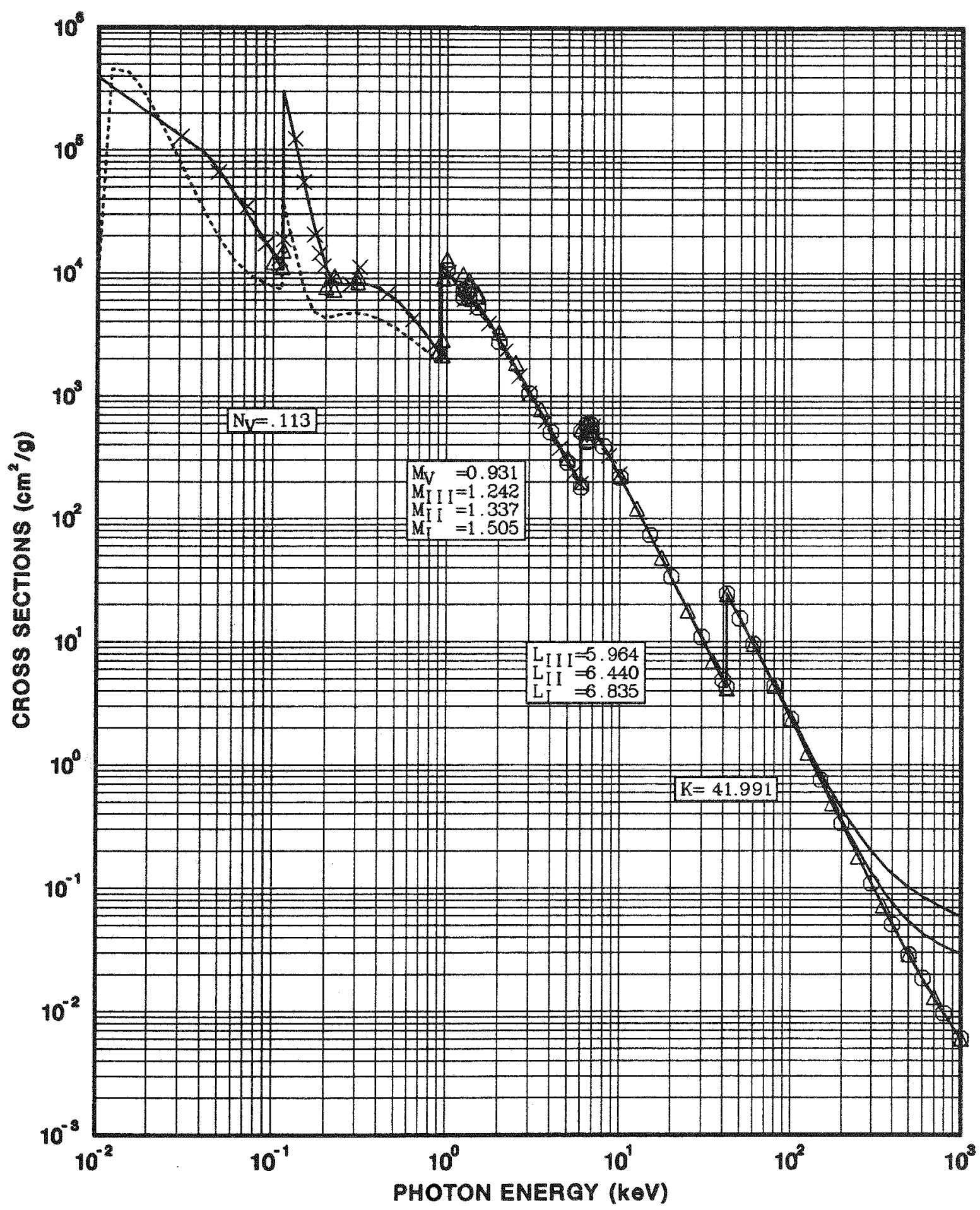

Barns/atom $=234.0 \times \mathrm{cm}^{2} / \mathrm{g}$ 


\section{NEODYMIUM 60}

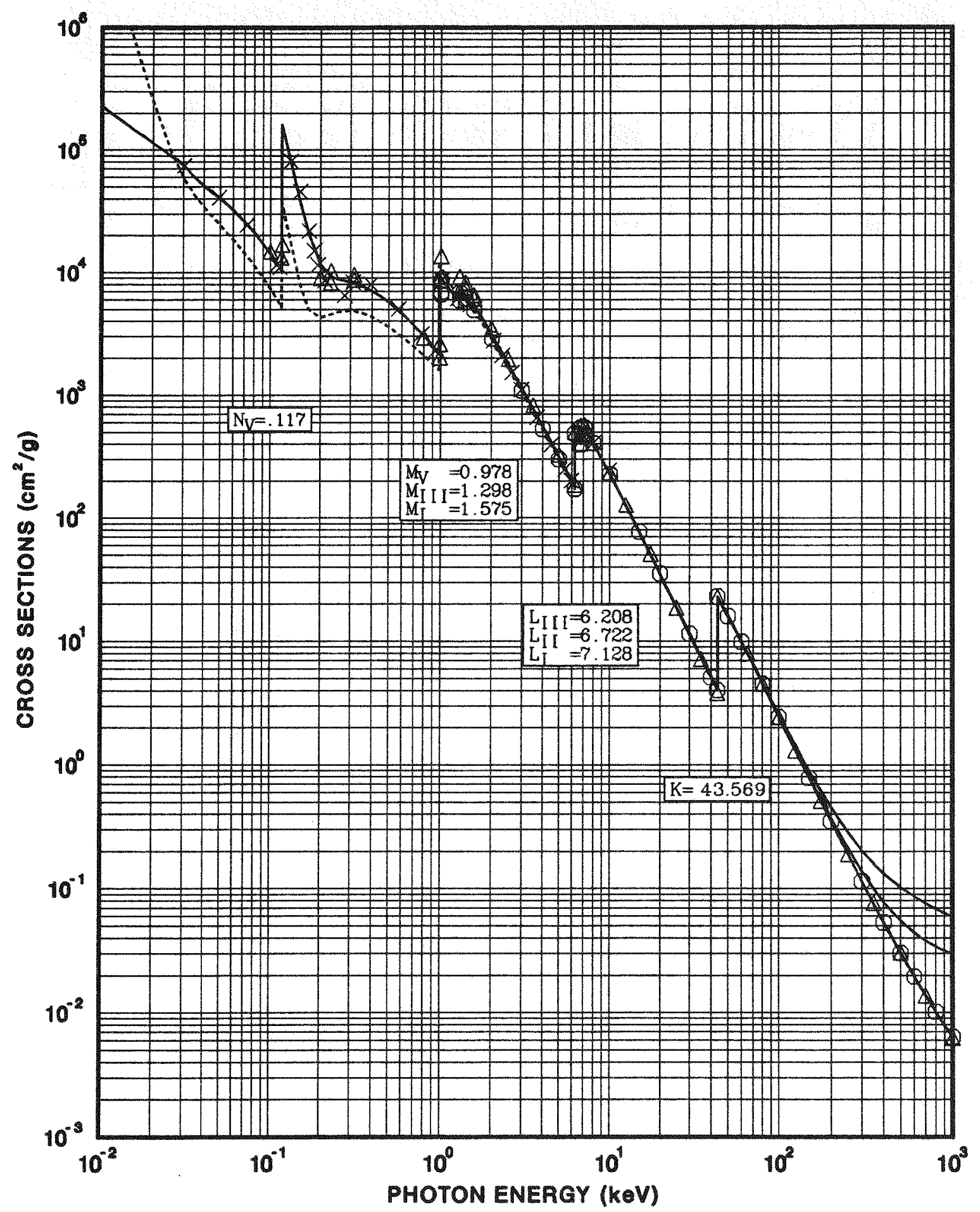

Barns/atom $=239.5 \times \mathrm{cm}^{2} / \mathrm{g}$ 
PROMETHIUM 61

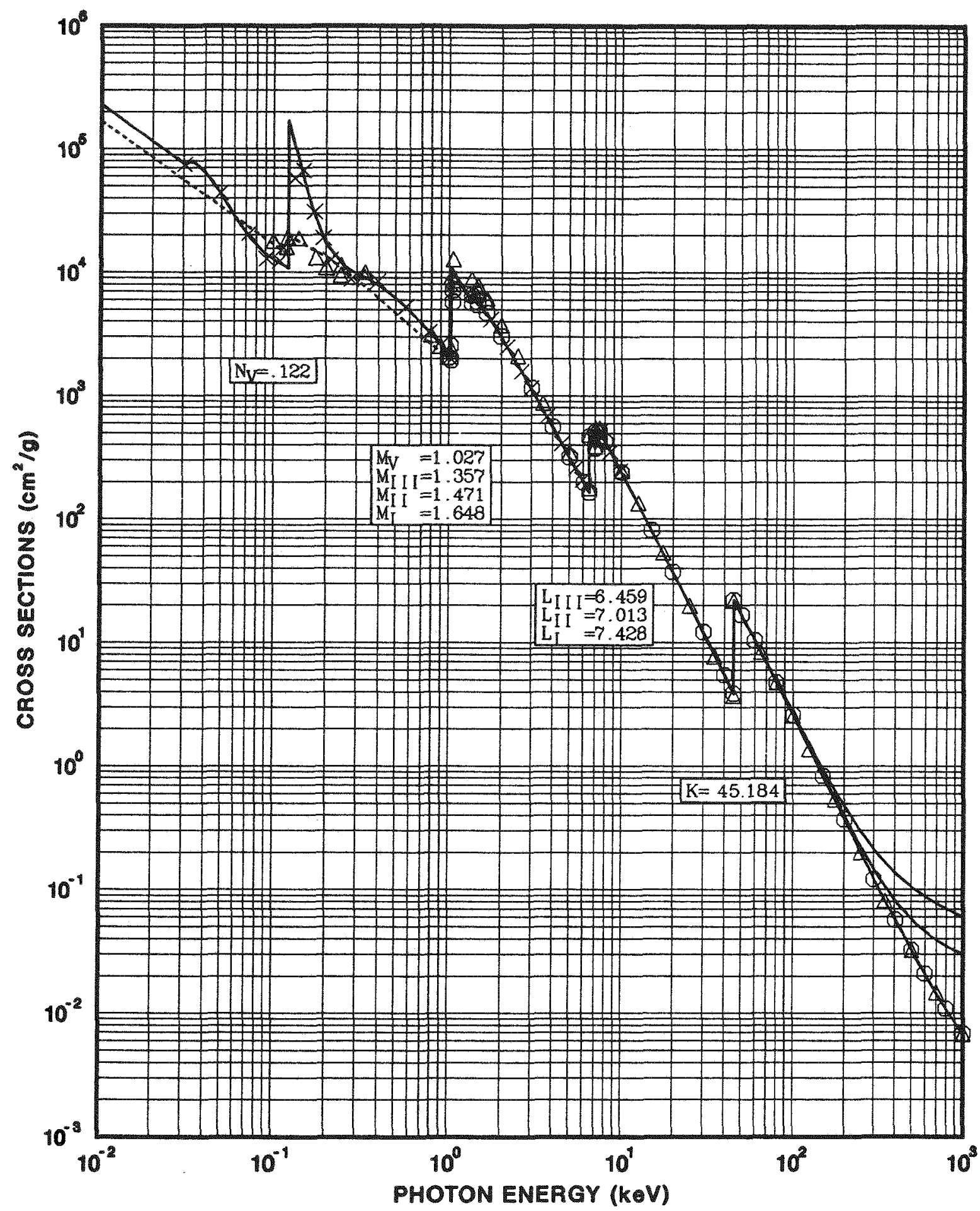

Barns/atom $=240.8 \times \mathrm{cm}^{2} / \mathrm{g}$ 


\section{SAMARIUM 62}

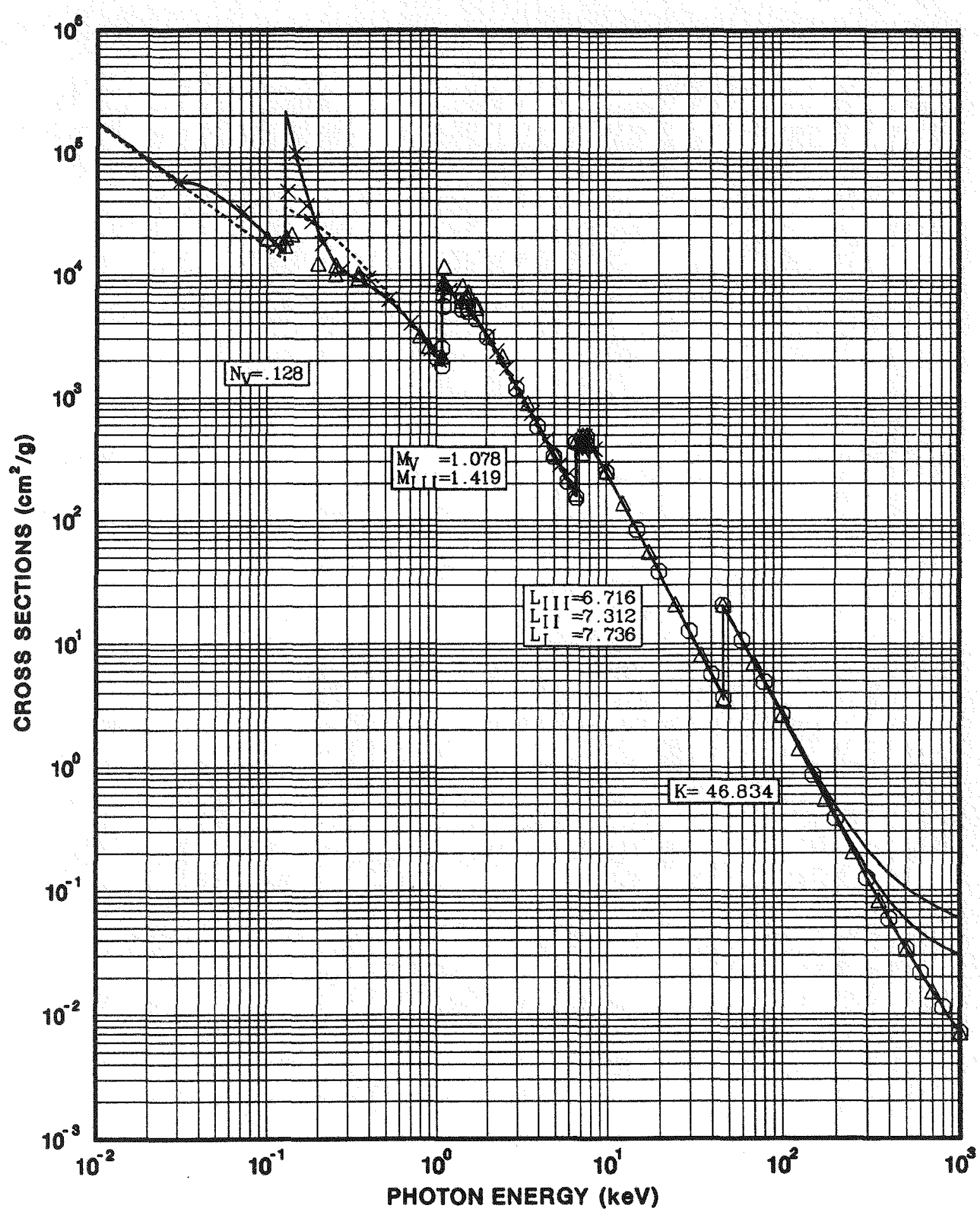

Barns/atom $=249.7 \times \mathrm{cm}^{2} / \mathrm{g}$ 


\section{EUROPIUM 63}

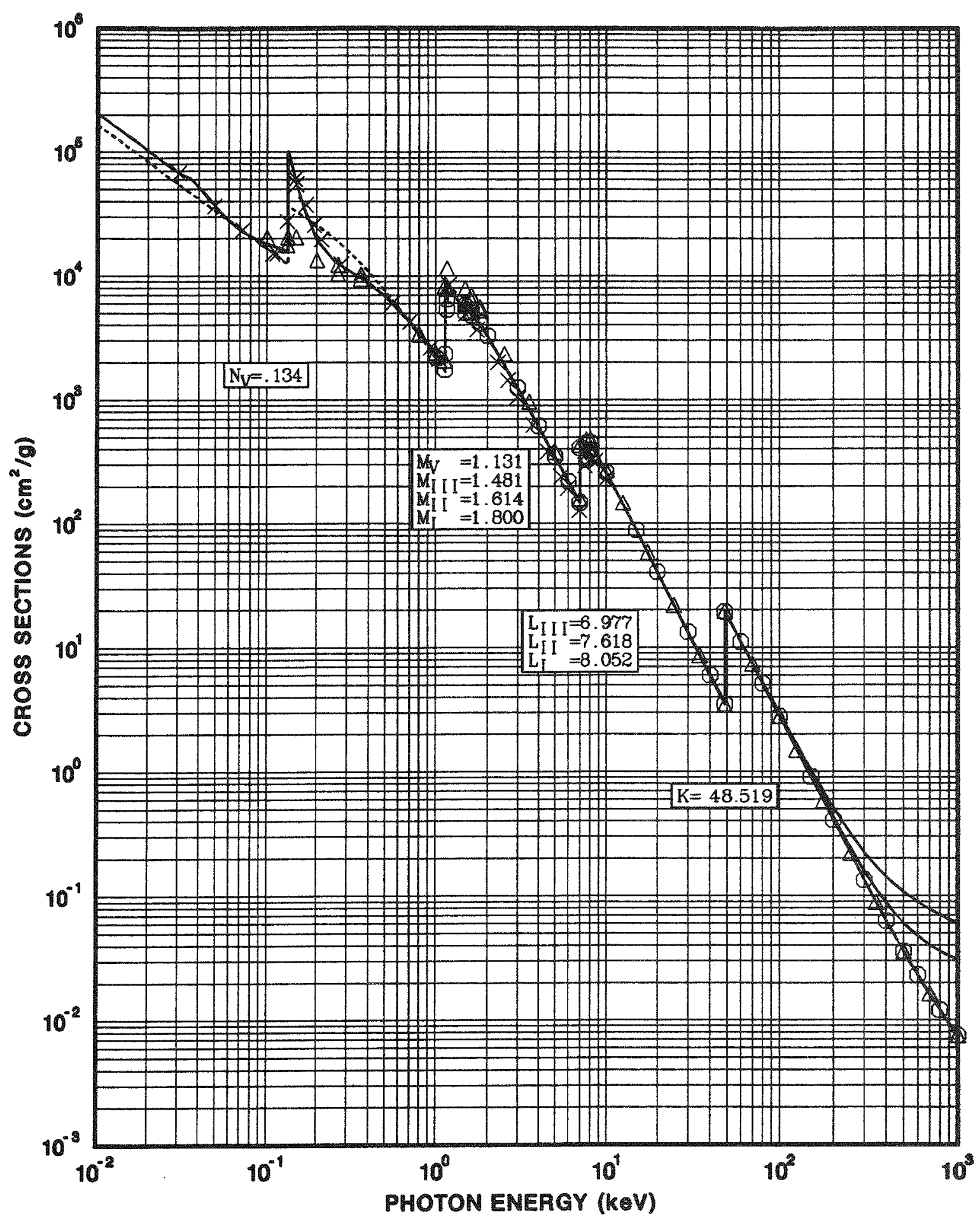

Barns/atom $=252.3 \times \mathrm{cm}^{2} / \mathrm{g}$ 


\section{GADOLINIUM 64}

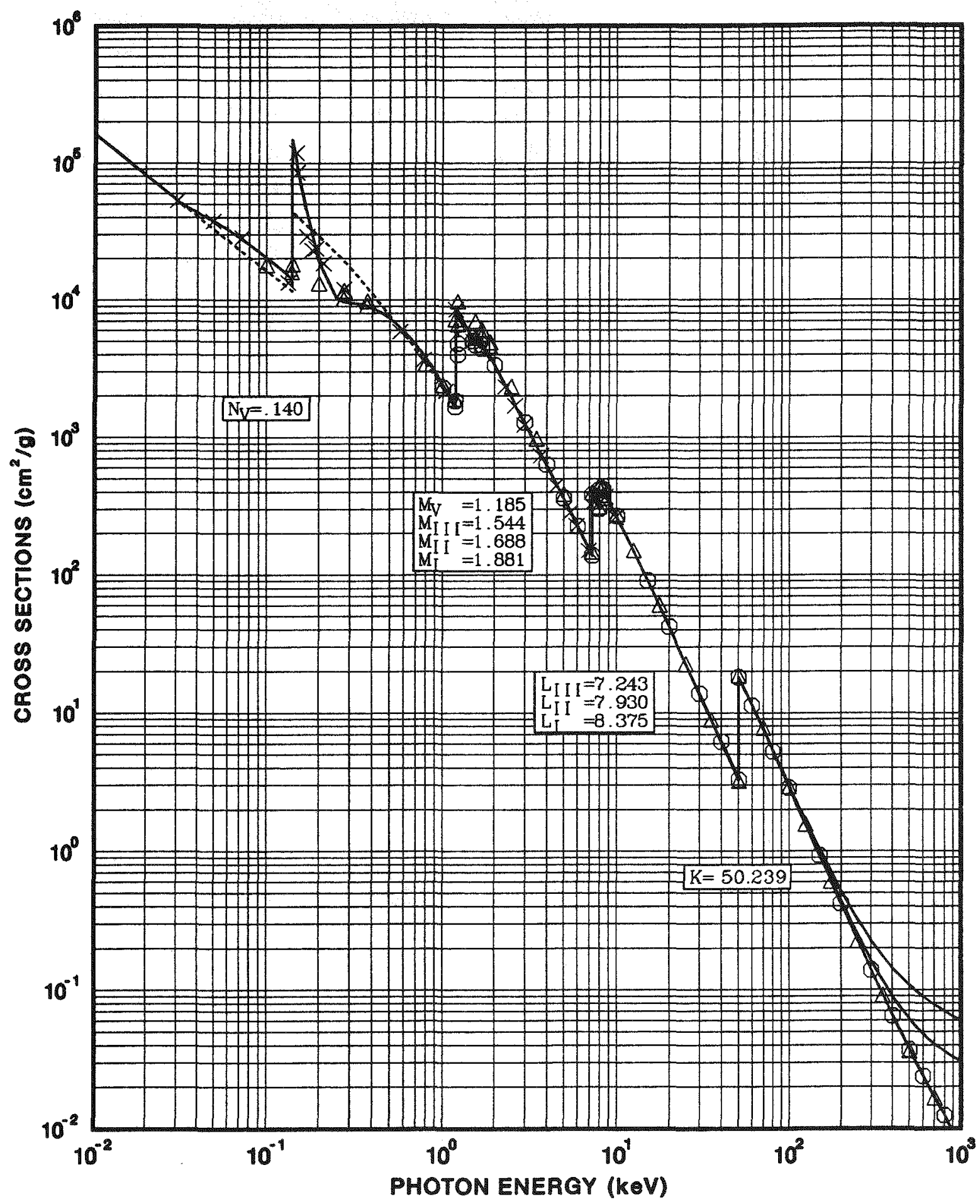

Barns/atom $=261.1 \times \mathrm{cm}^{2} / \mathrm{g}$ 
TERBIUM 65

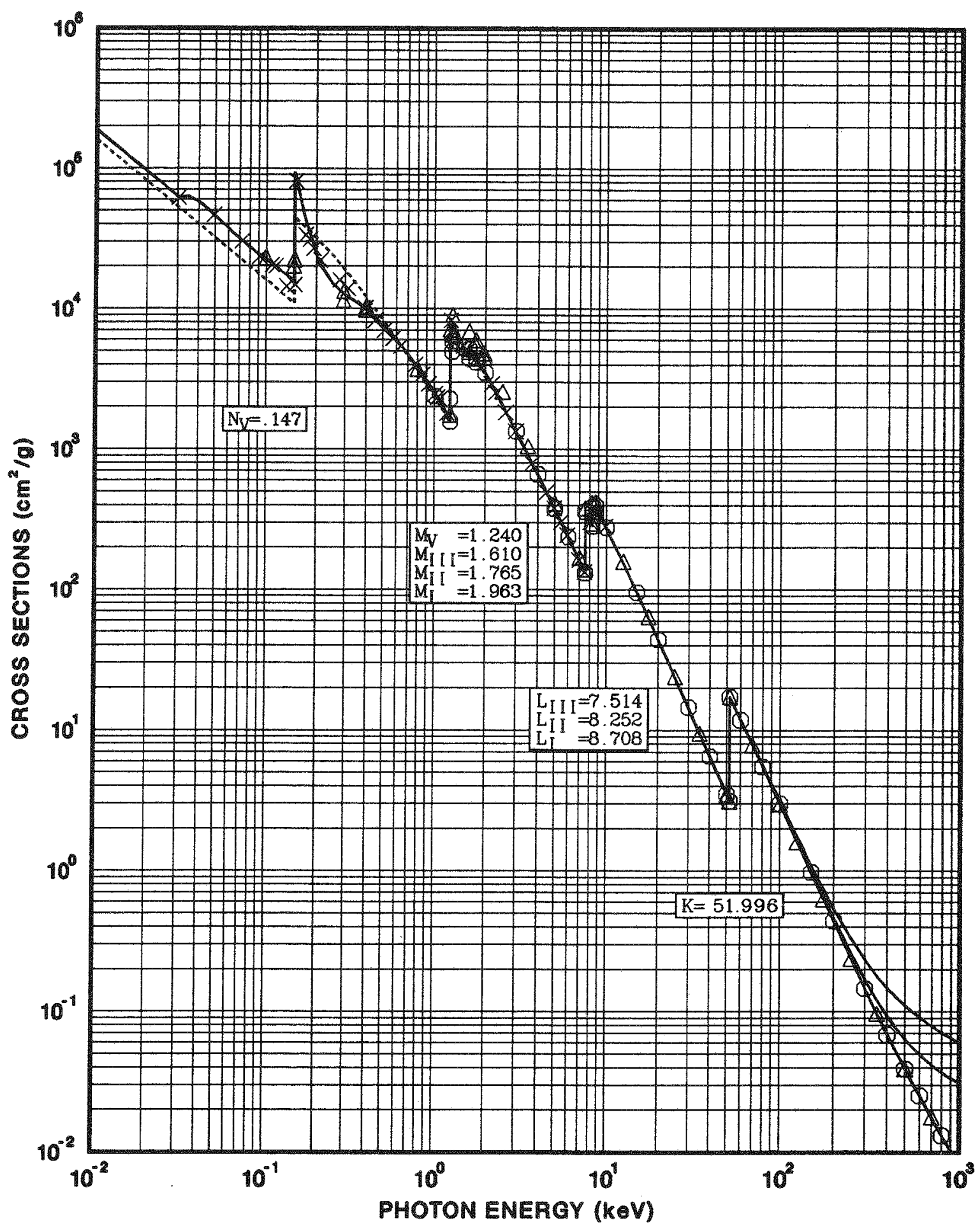

Barns/atom $=263.9 \times \mathrm{cm}^{2} / \mathrm{g}$ 
DYSPROSIUM 66

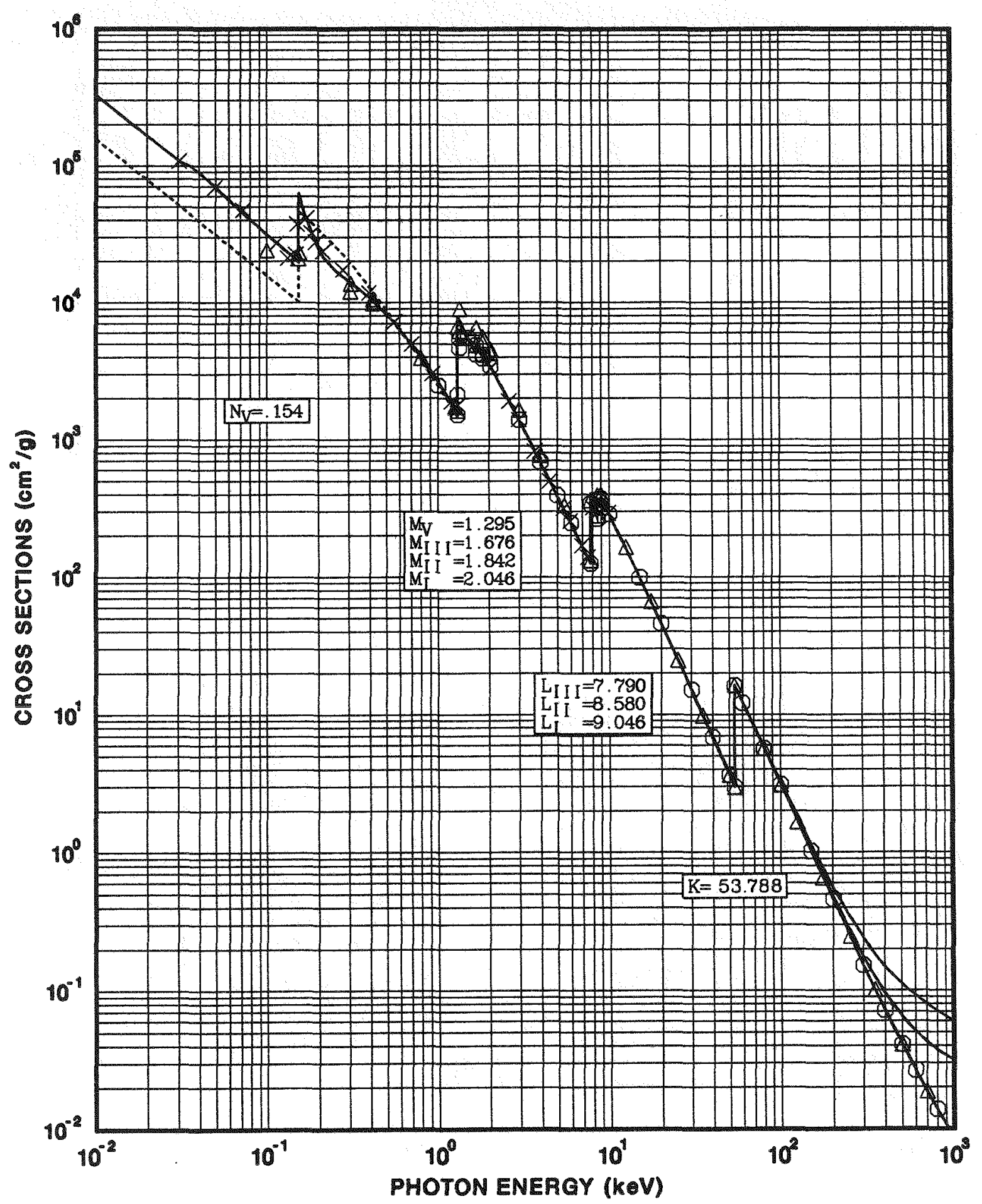

Barns/atom $=269.8 \times \mathrm{cm}^{2} / \mathrm{g}$ 


\section{HOLMIUM 67}

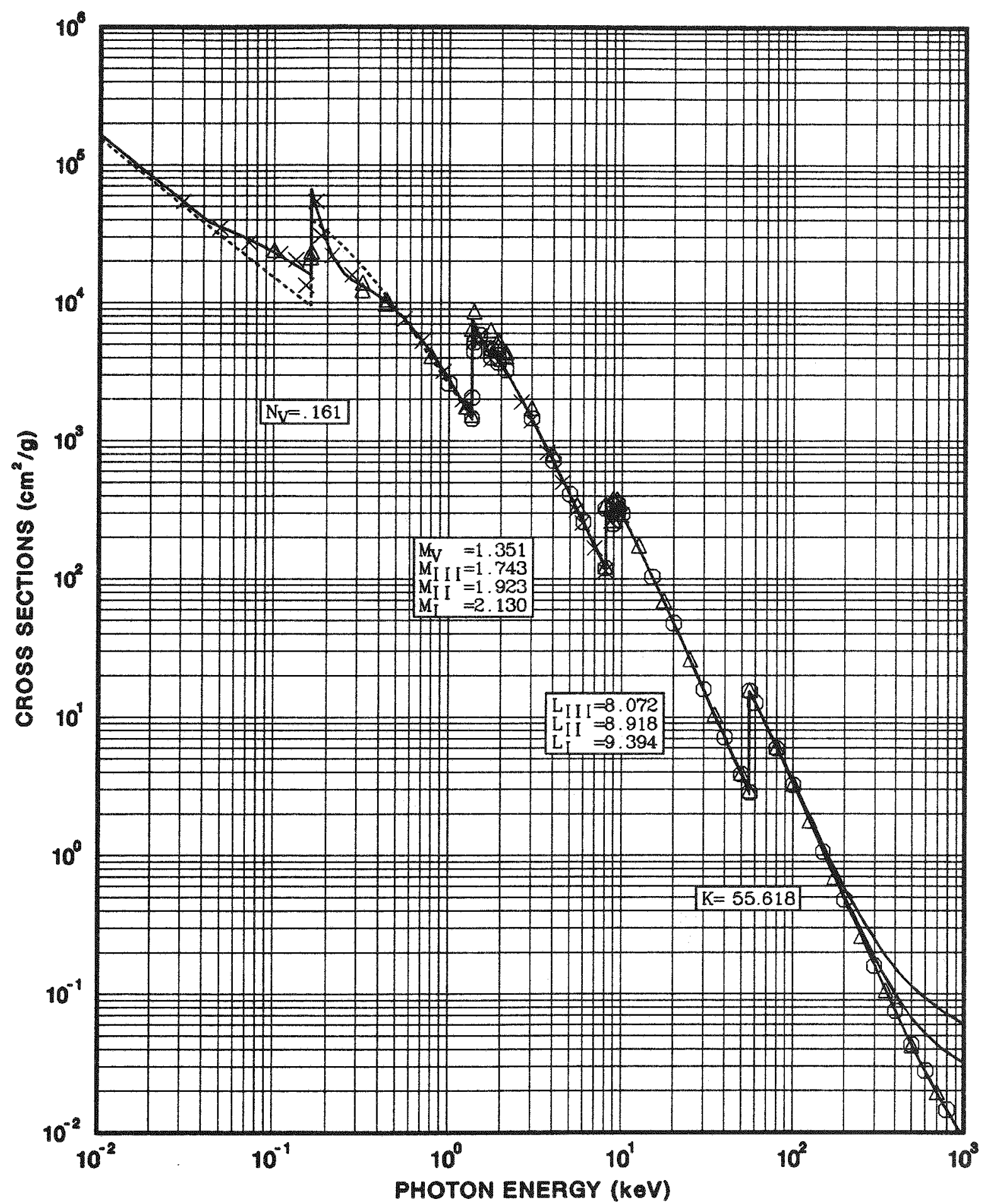

Barns/atom $=273.9 \times \mathrm{cm}^{2} / \mathrm{g}$ 


\section{ERBIUM 68}

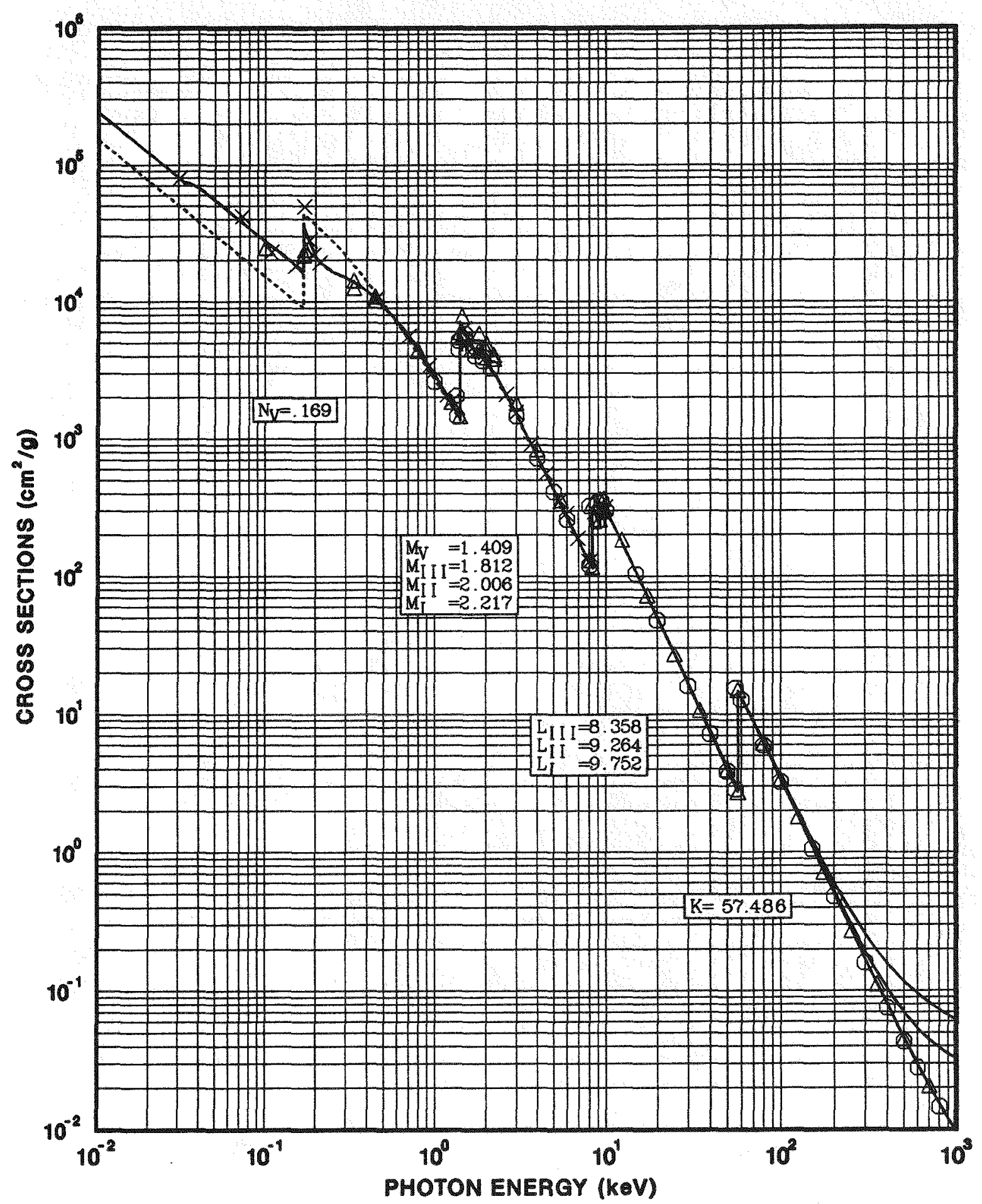

Barns/atom $=277.7 \times \mathrm{cm}^{2} / \mathrm{g}$ 
THULIUM 69

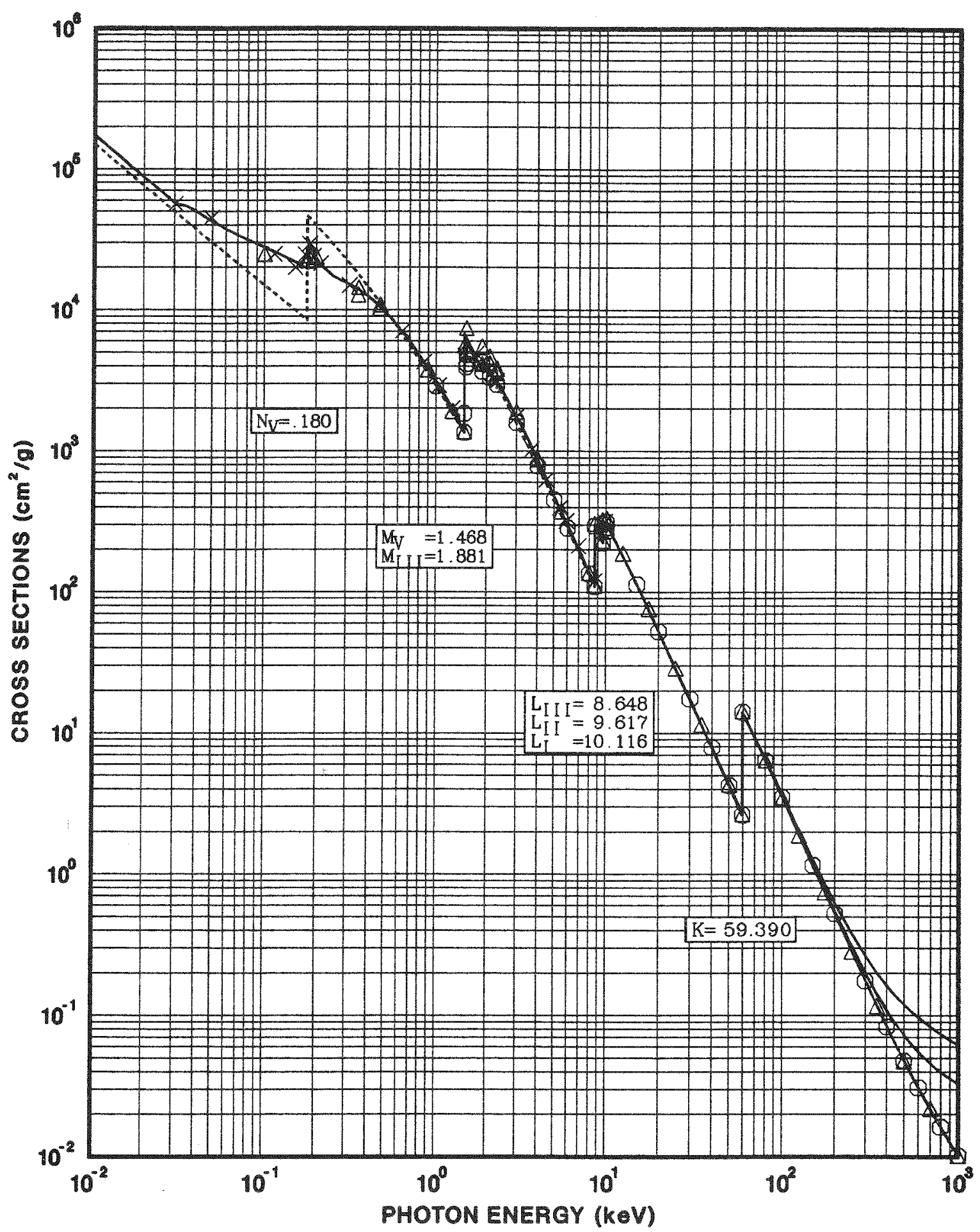

Barns/atom $=280.5 \times \mathrm{cm}^{2} / \mathrm{g}$ 


\section{YTTERBIUM 70}

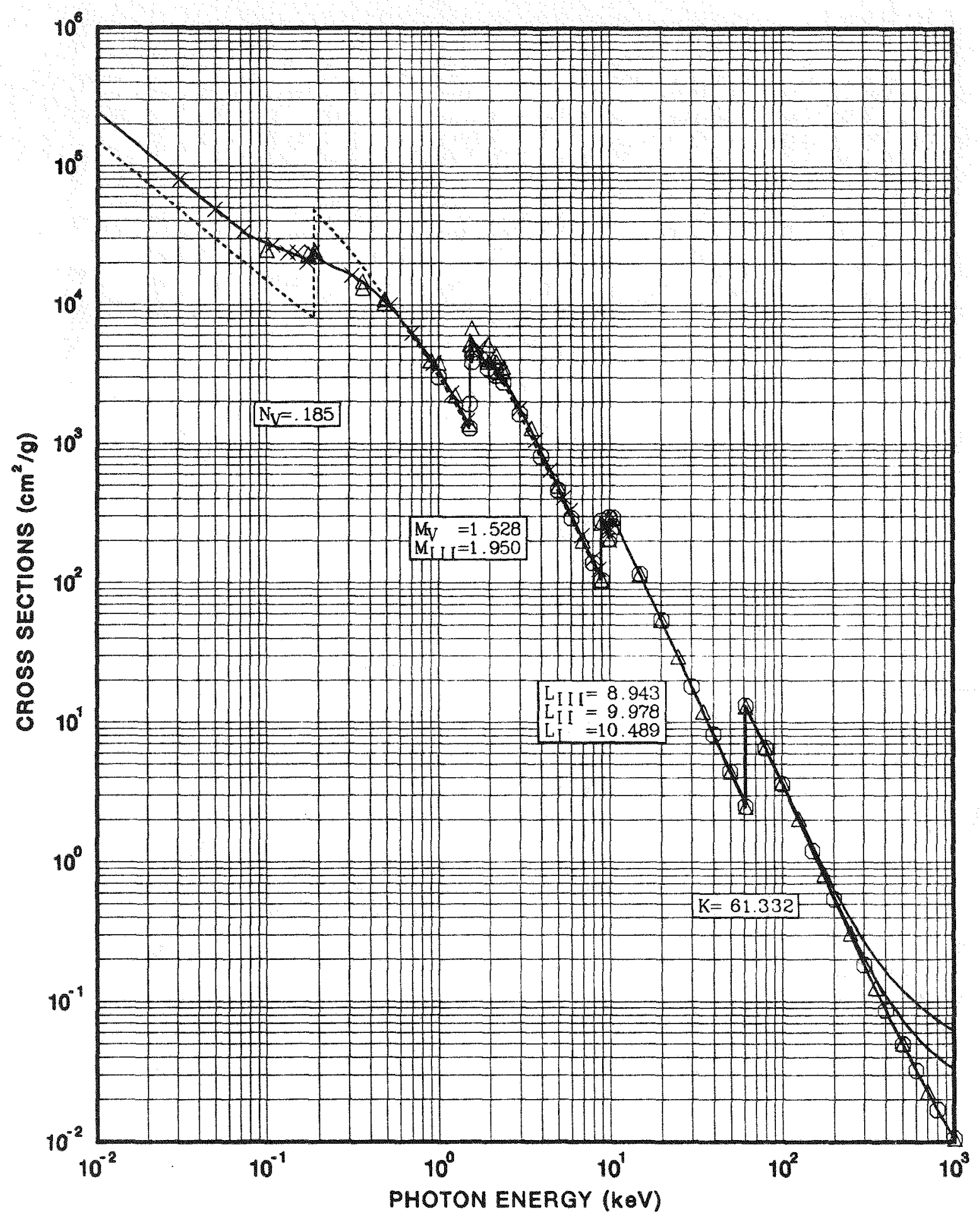

Barns'atom $=287.3 \times \mathrm{cm}^{2} / \mathrm{g}$ 


\section{LUTETIUM 71}

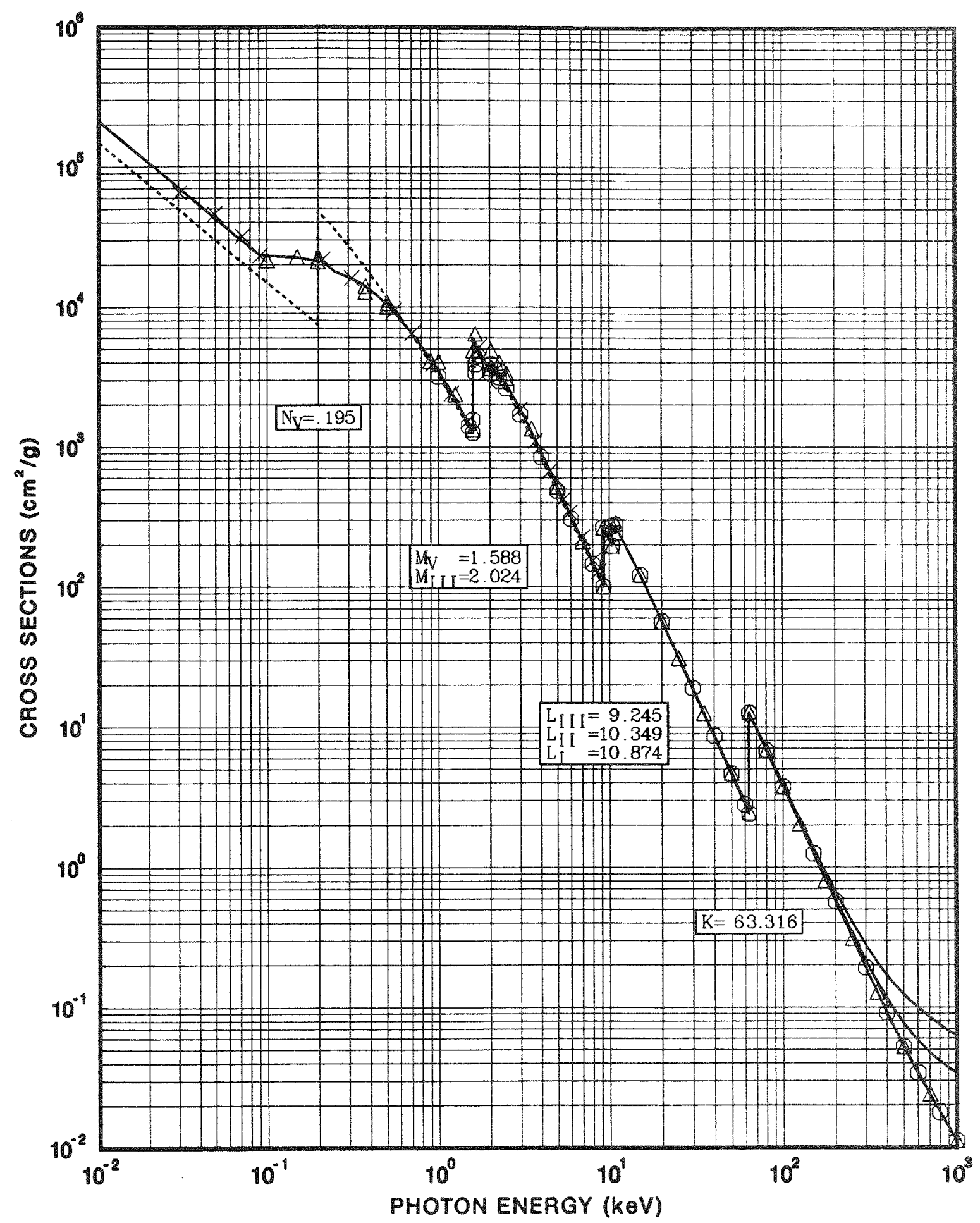

Barns/atom $=290.5 \times \mathrm{cm}^{2} / \mathrm{g}$ 


\section{HAFNIUM 72}

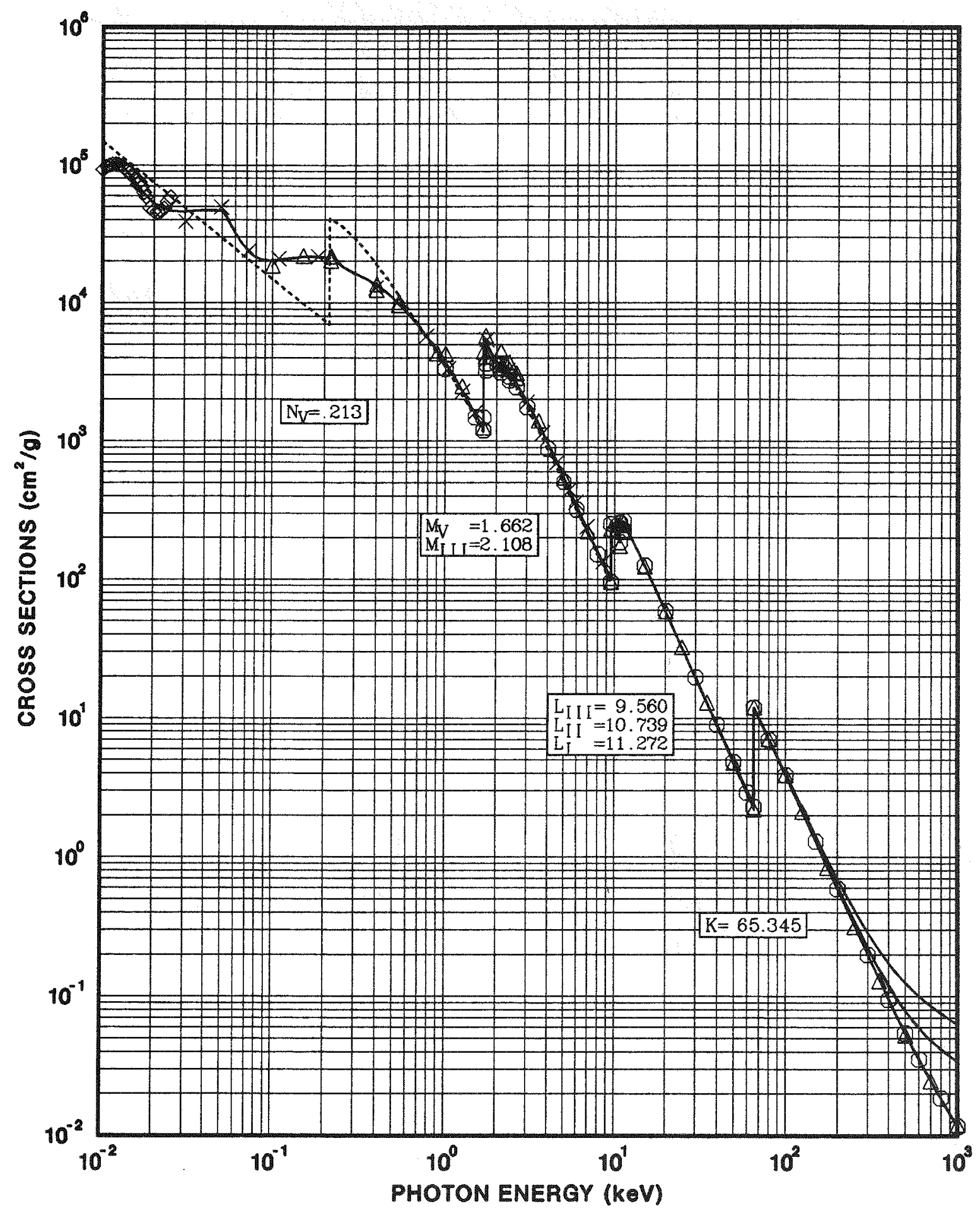

Barns/atom $=296.4 \times \mathrm{cm}^{2} / \mathrm{g}$ 
TANTALUM 73

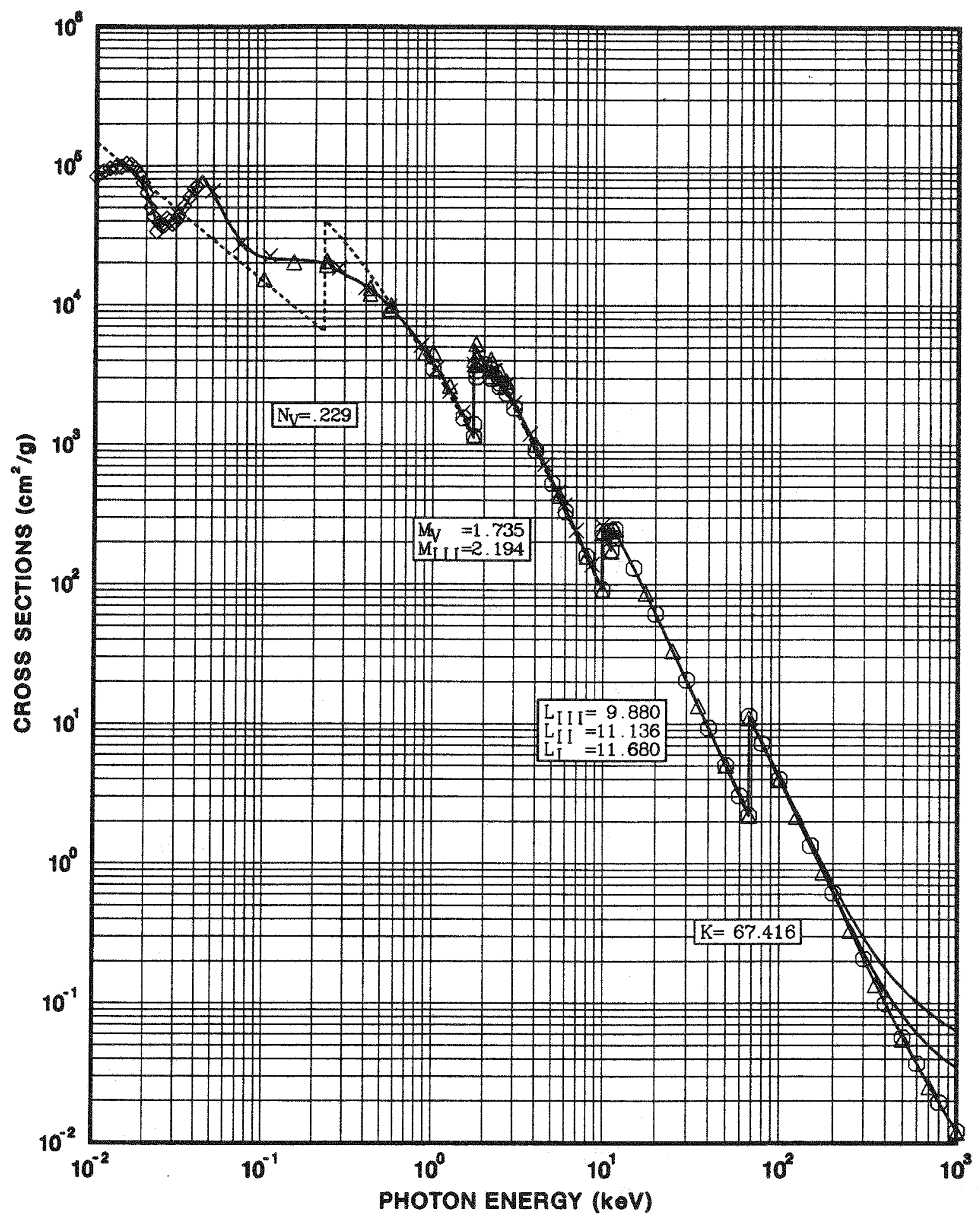

Barns/atom $=300.5 \times \mathrm{cm}^{2} / \mathrm{g}$ 


\section{TUNGSTEN 74}

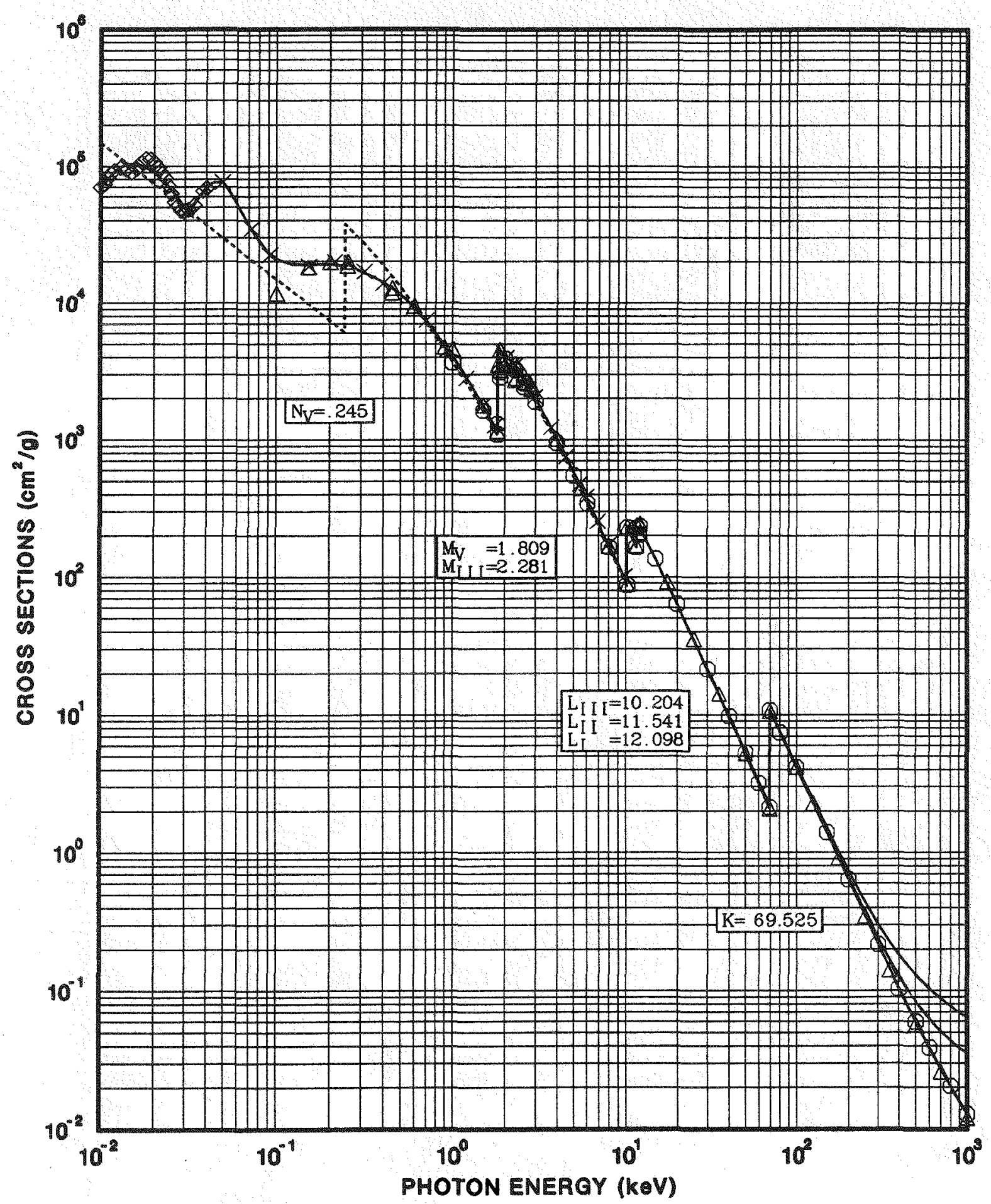

Barns/atom $=305.3 \times \mathrm{cm}^{2} / \mathrm{g}$ 
RHENIUM 75

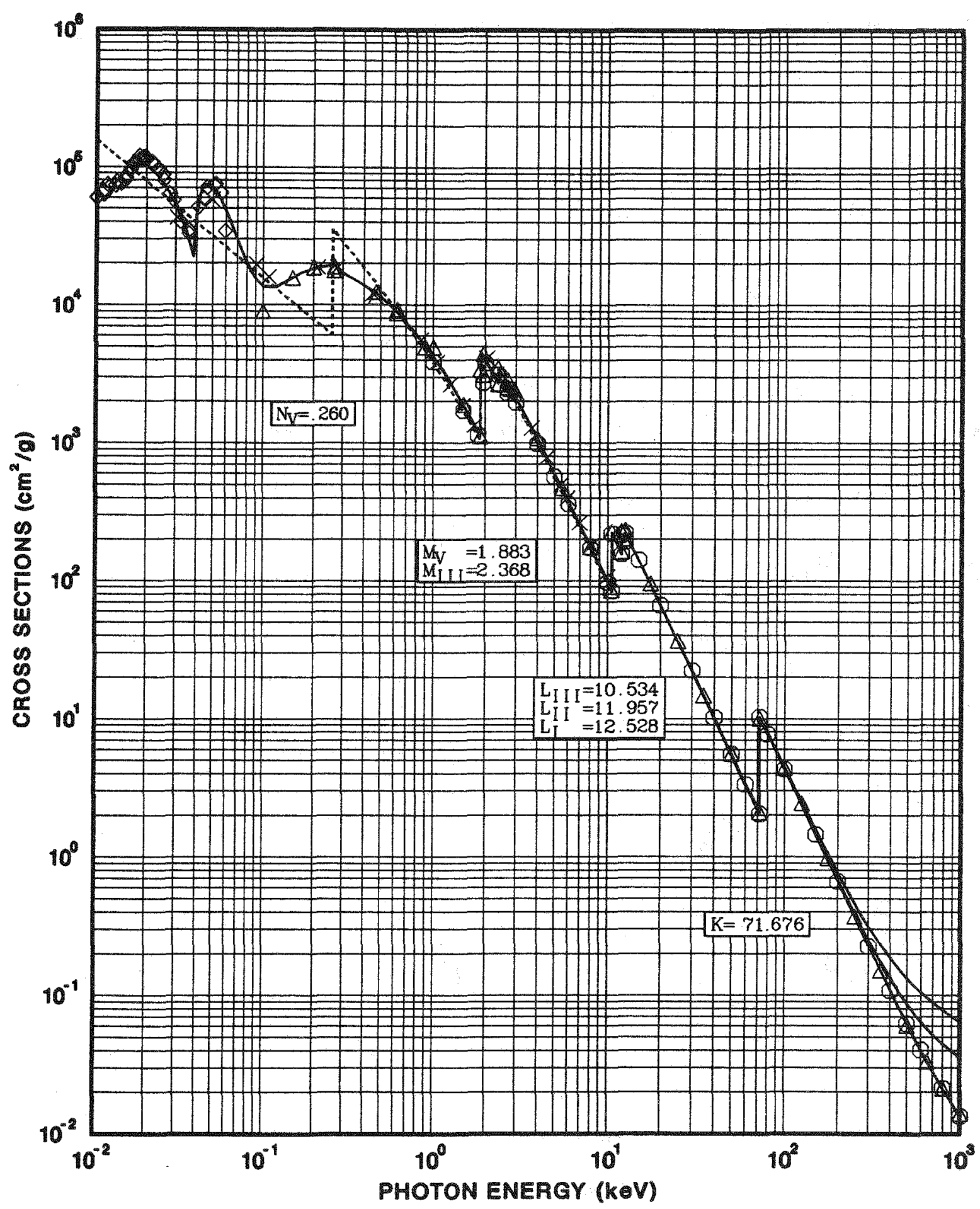

Barns/atom $=309.2 \times \mathrm{cm}^{2} / \mathrm{g}$ 
OSMIUM 76

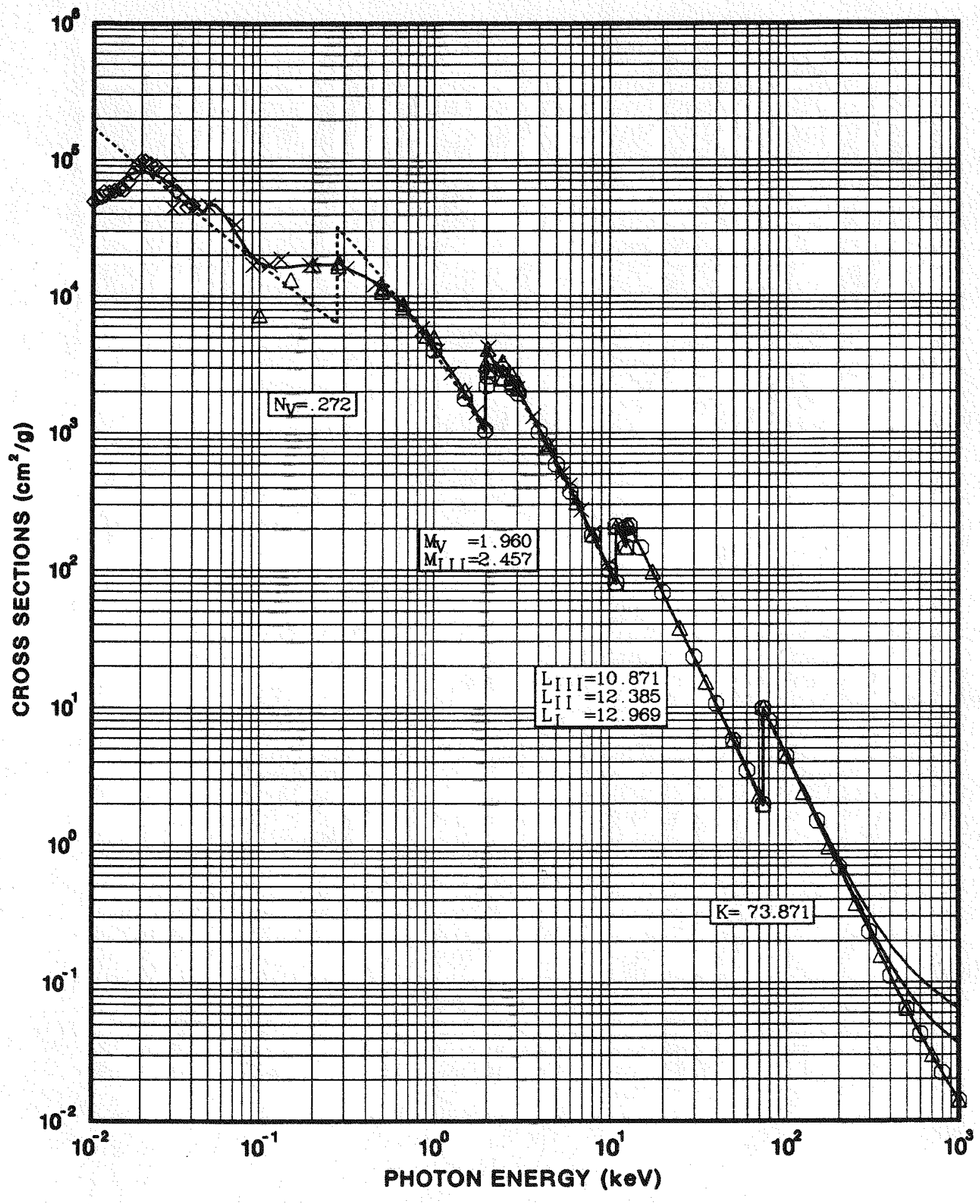

Barns/atom $=315.8 \times \mathrm{cm}^{2} / \mathrm{g}$ 
IRIDIUM 77

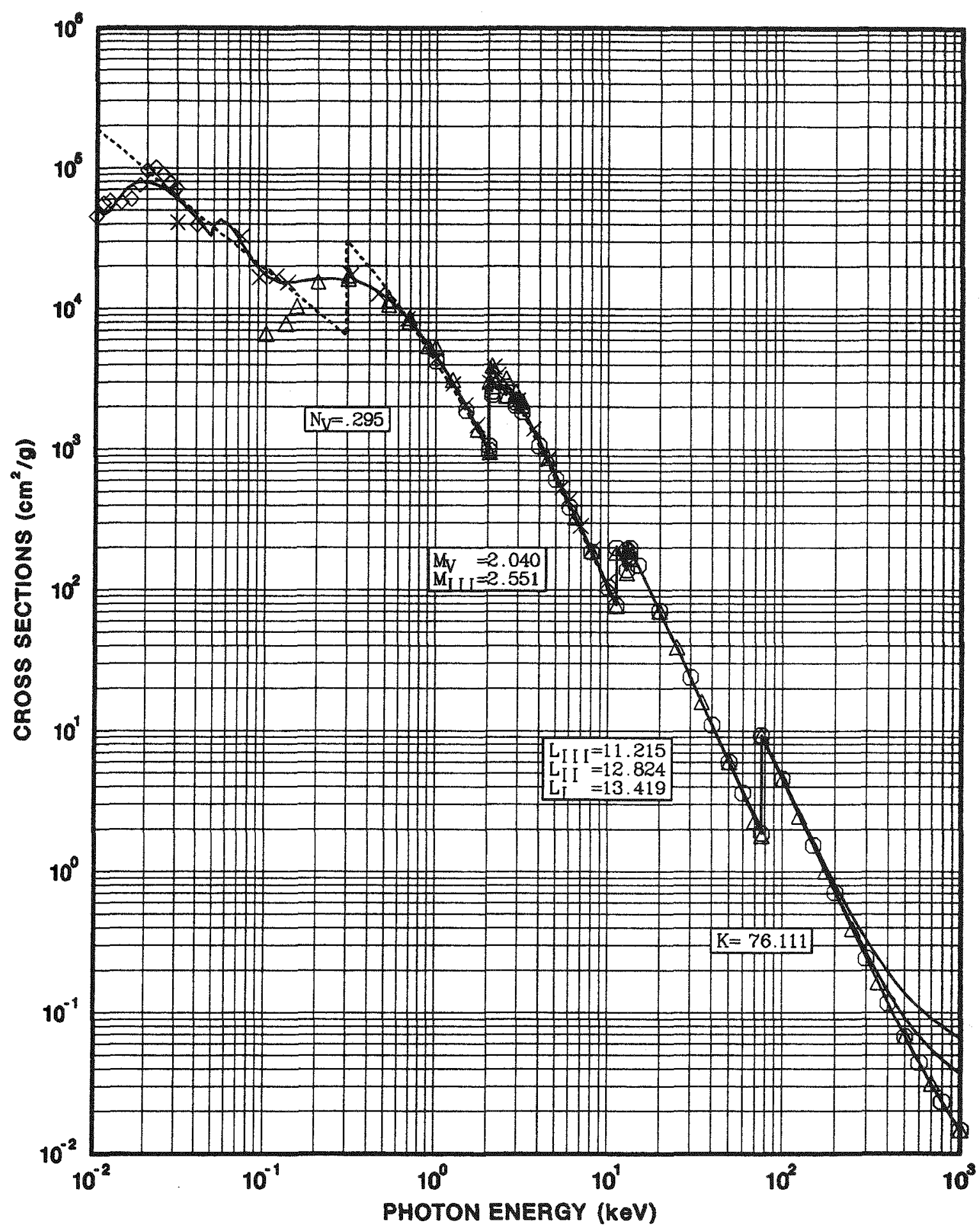

Barns/atom $=319.2 \times \mathrm{cm}^{2} / \mathrm{g}$ 
PLATINUM 78

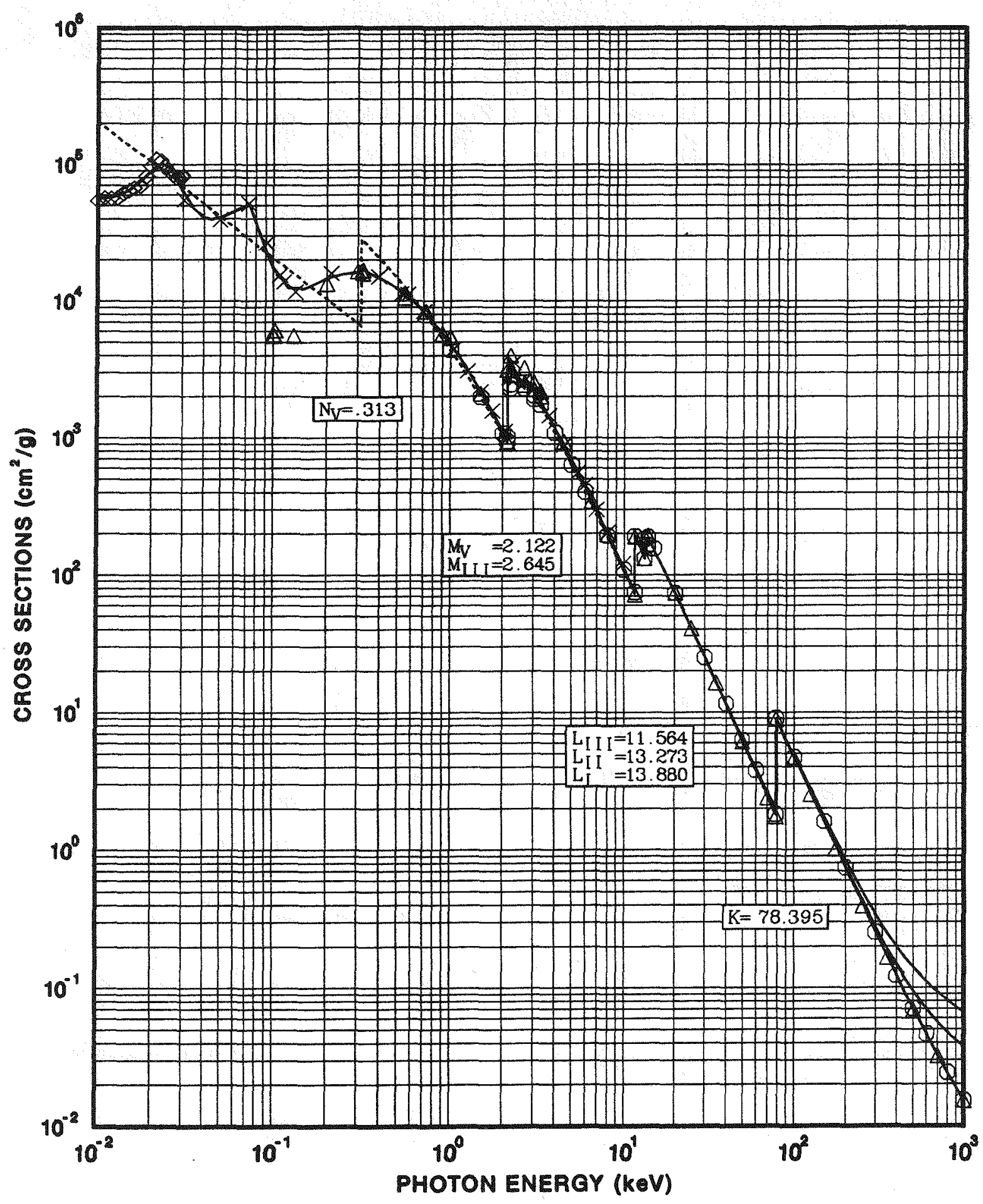

Barns/atom $=323.9 \times \mathrm{cm}^{2} / \mathrm{g}$ 
GOLD 79

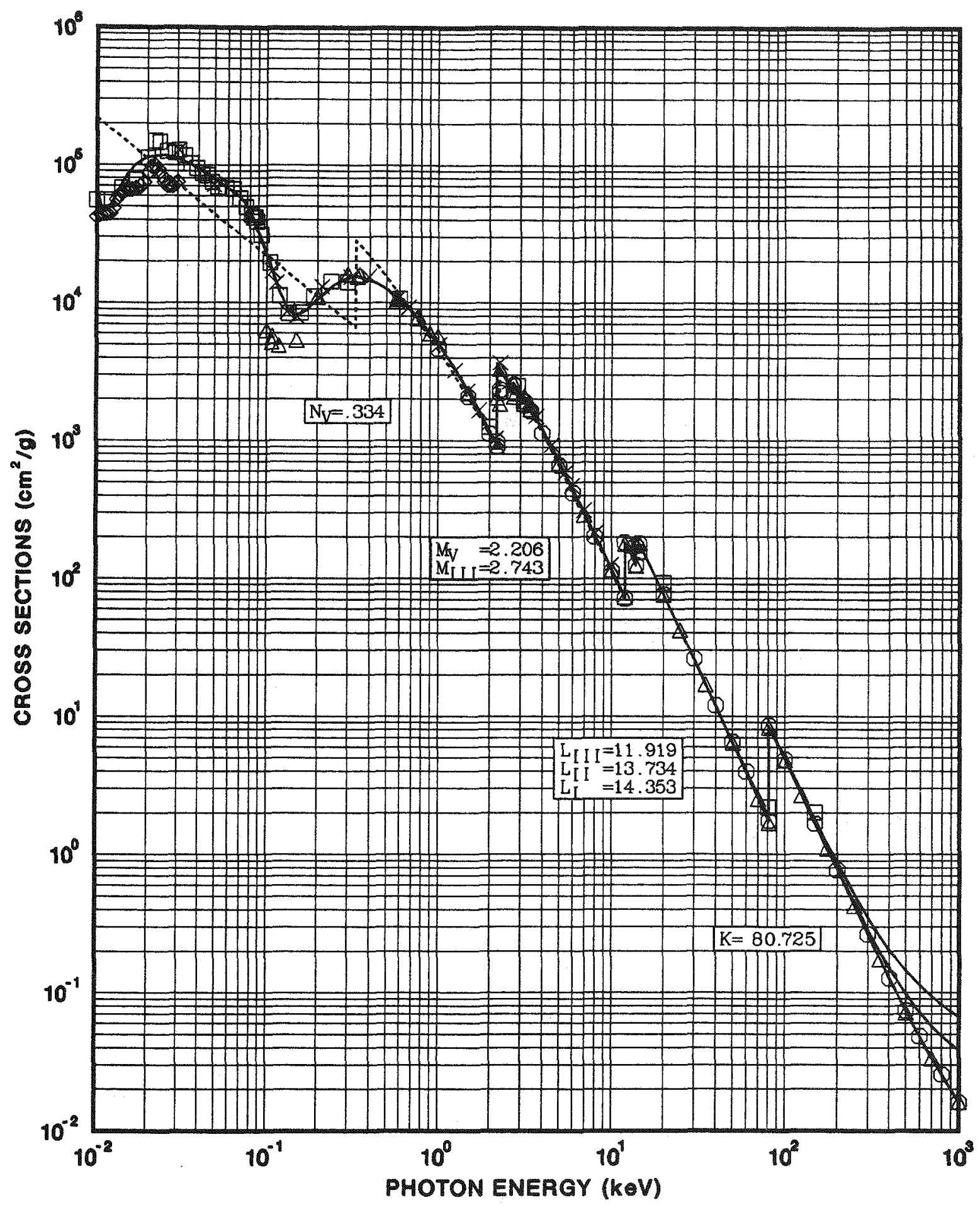

Barns/atom $=327.1 \times \mathrm{cm}^{2} / \mathrm{g}$ 


\section{MERCURY 80}

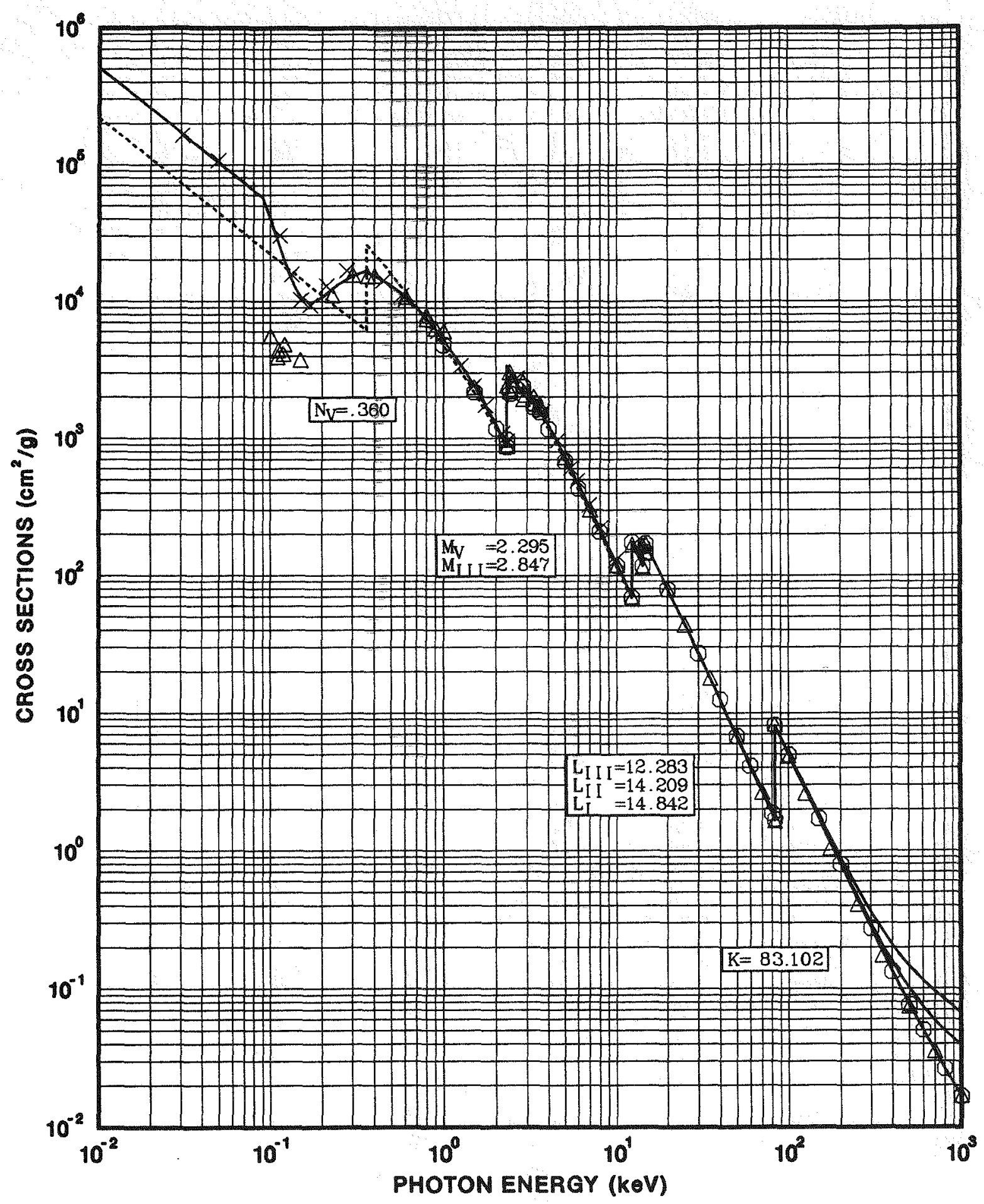

Barns/atom $=333.1 \times \mathrm{cm}^{2} / \mathrm{g}$ 
THALLIUM 81

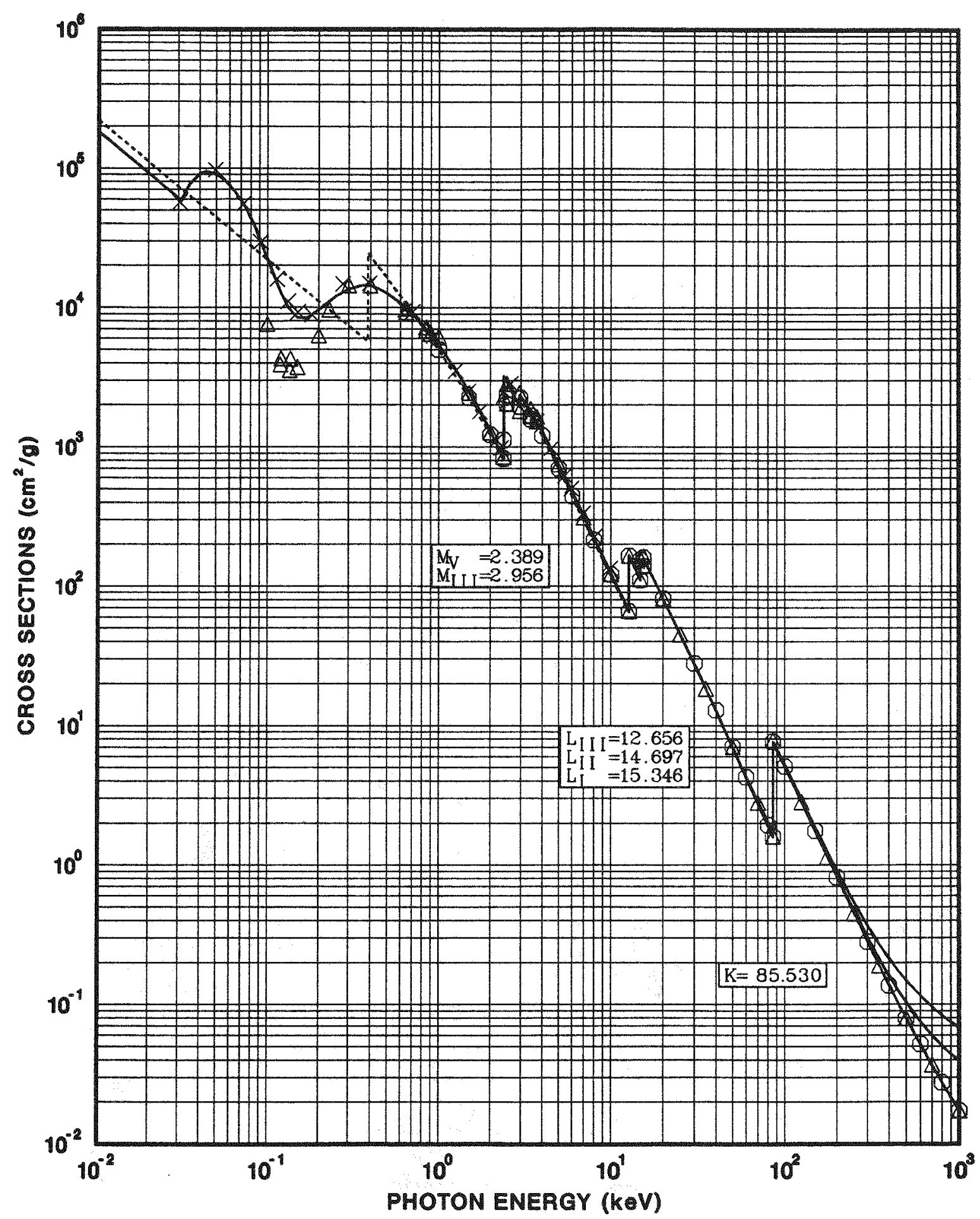

Barns/atom $=339.4 \times \mathrm{cm}^{2} / \mathrm{g}$ 
LEAD 82

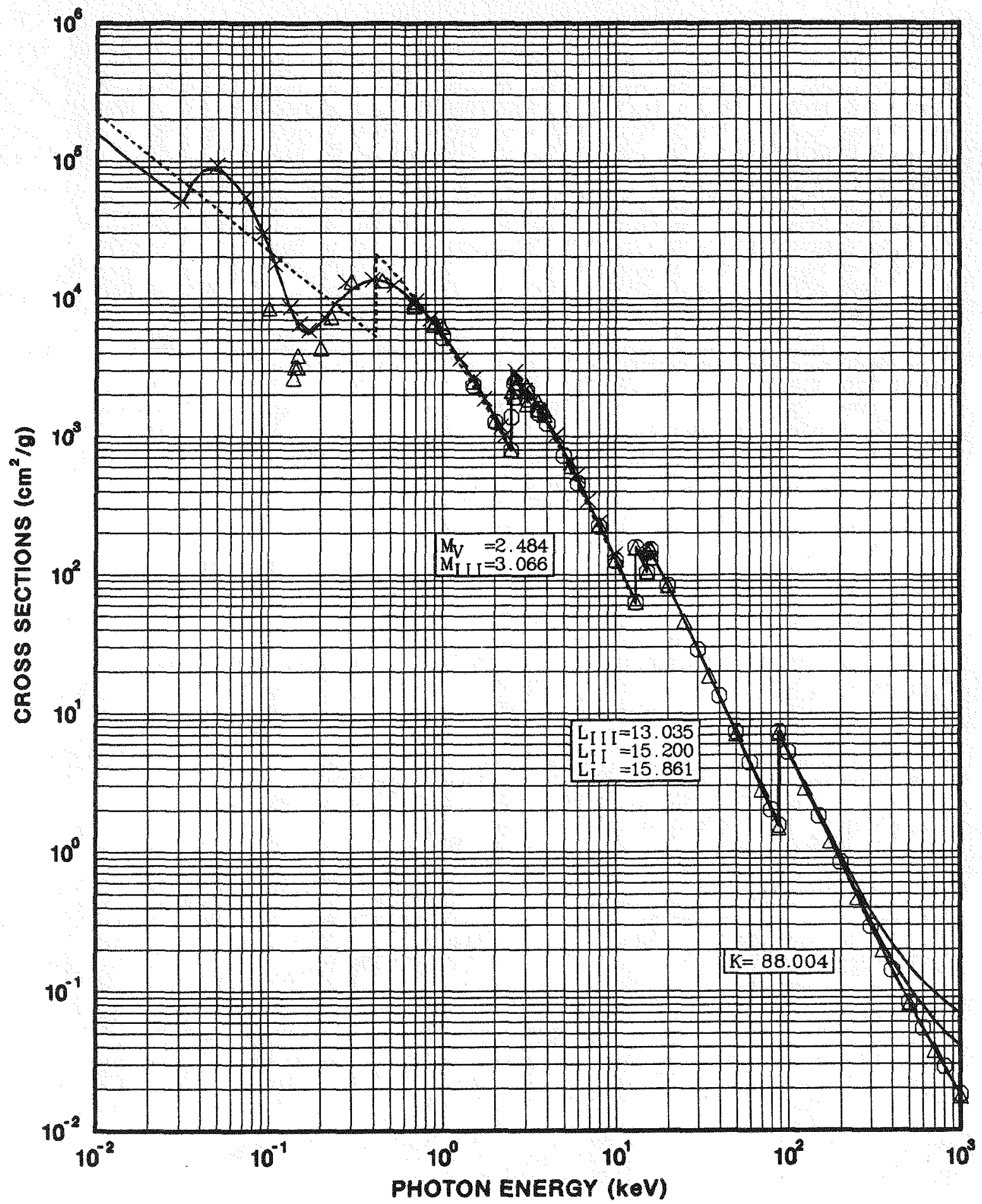

Barns/atom $=344.1 \times \mathrm{cm}^{2} / \mathrm{g}$ 


\section{BISMUTH 83}

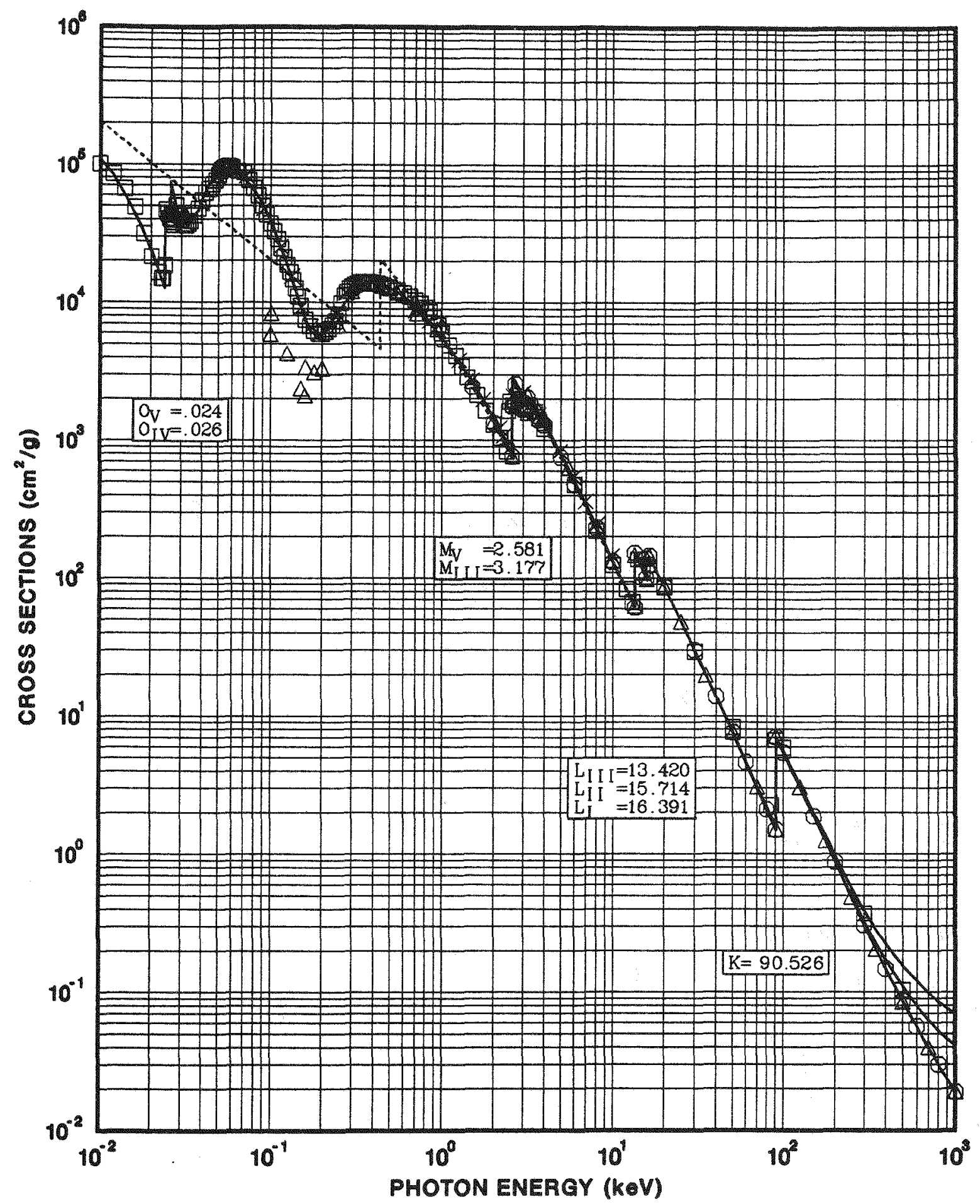

Barns/aton $=347.0 \times \mathrm{cm}^{2} / \mathrm{g}$ 
POLONIUM 84

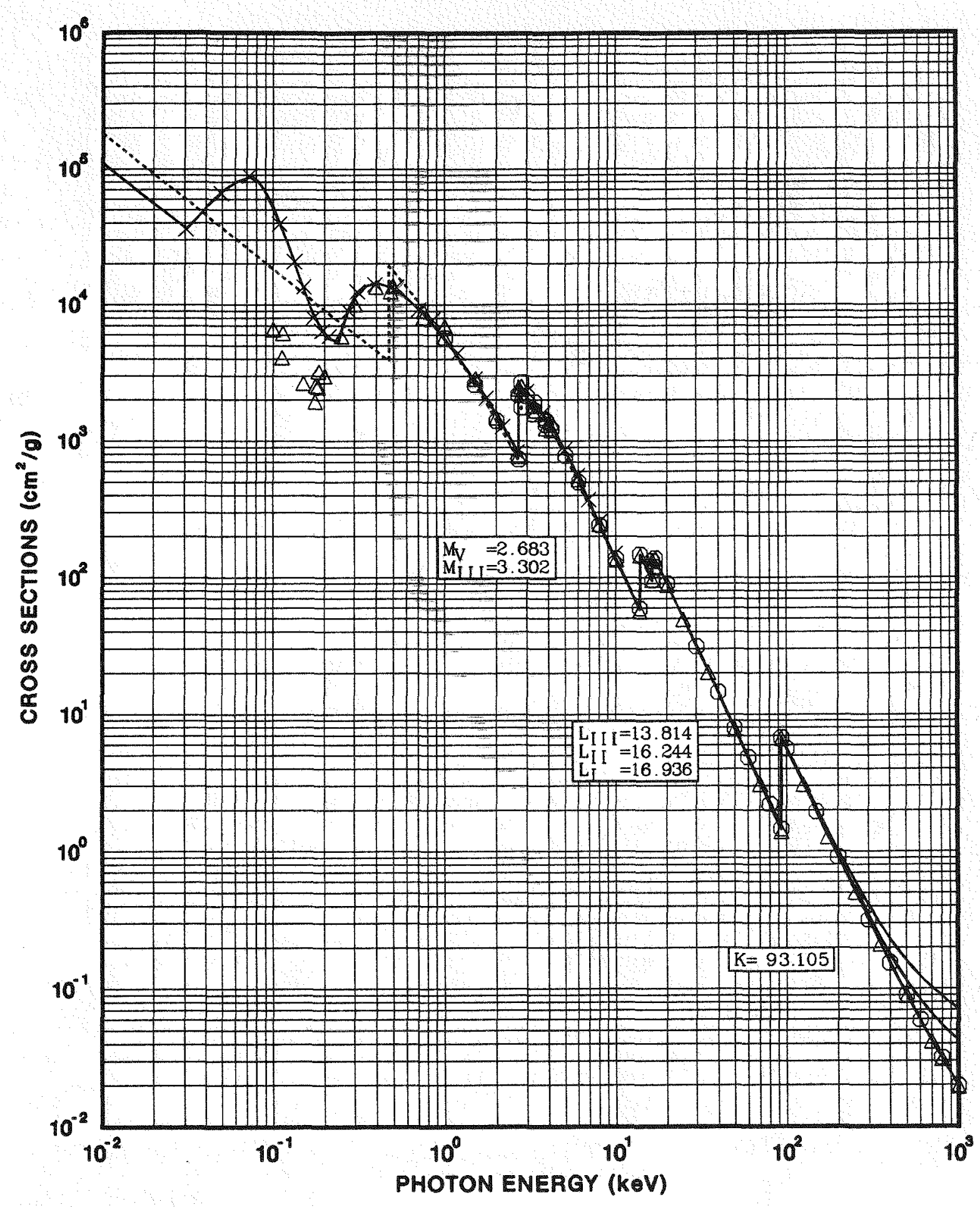

Barns/atom $=347.1 \times \mathrm{cm}^{2} / \mathrm{g}$ 


\section{ASTATINE 85}

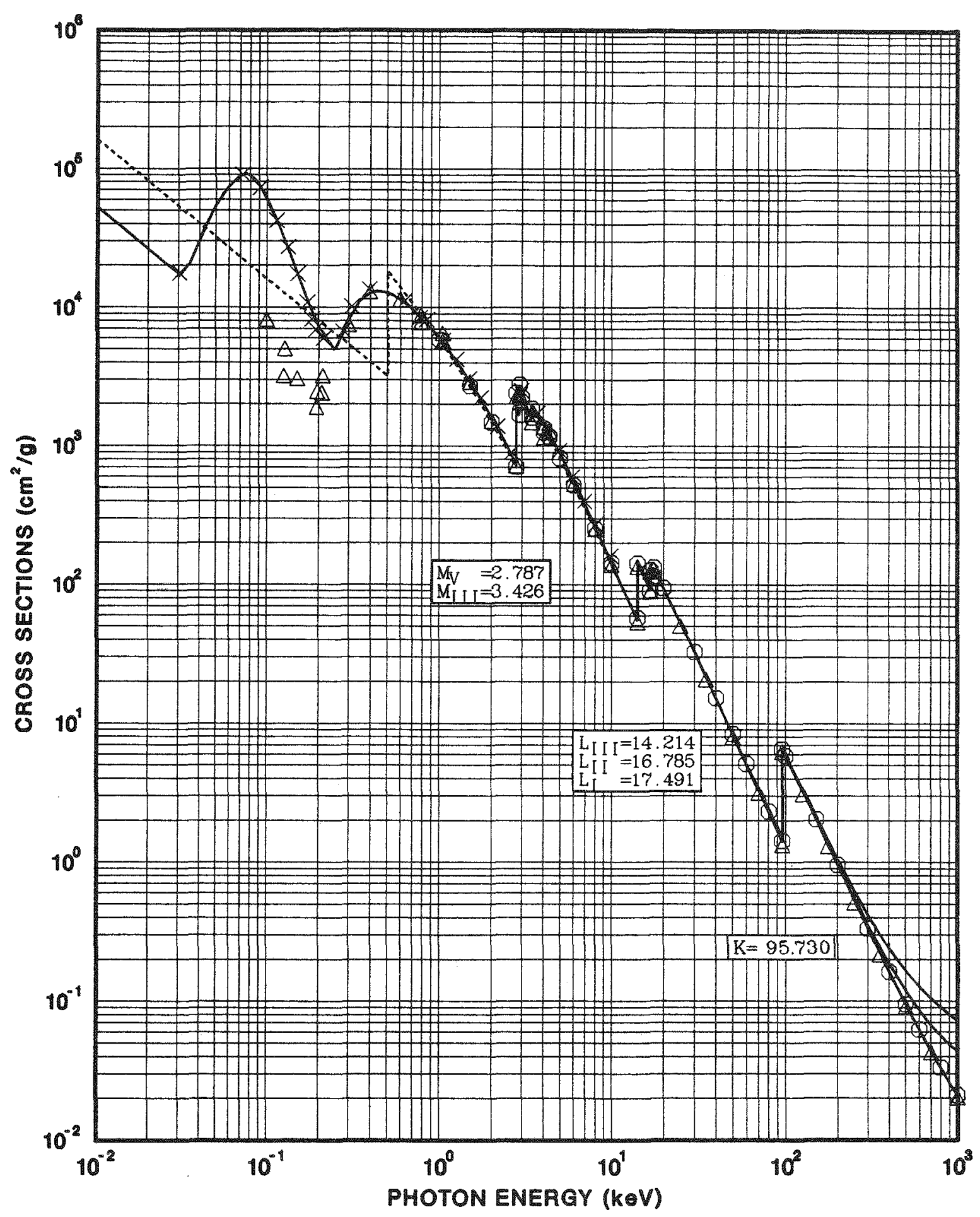

Barns/atom $=348.7 \times \mathrm{cm}^{2} / \mathrm{g}$ 
RADON 86

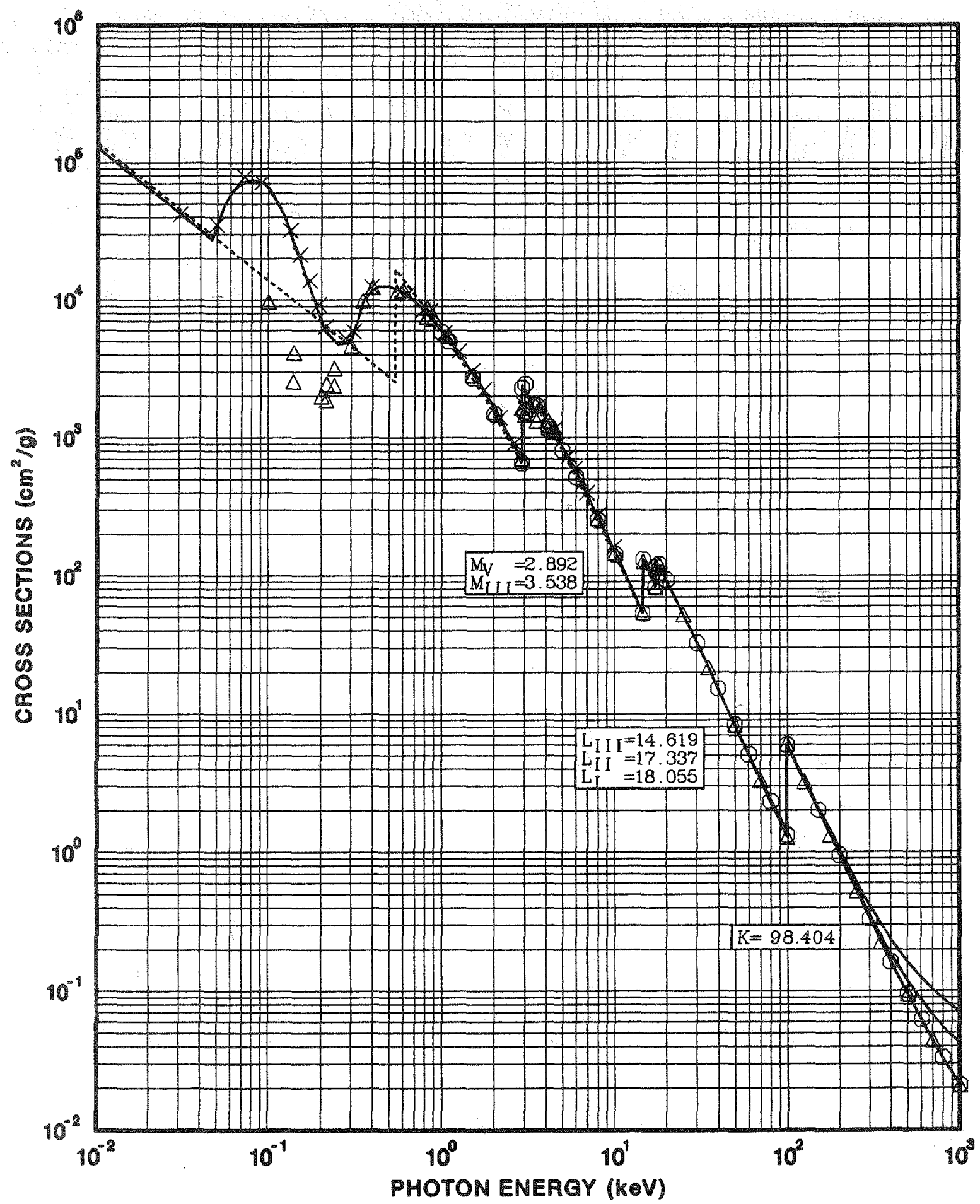

Barns/atom $=368.6 \times \mathrm{cm}^{2} / \mathrm{g}$ 
FRANCIUM 87

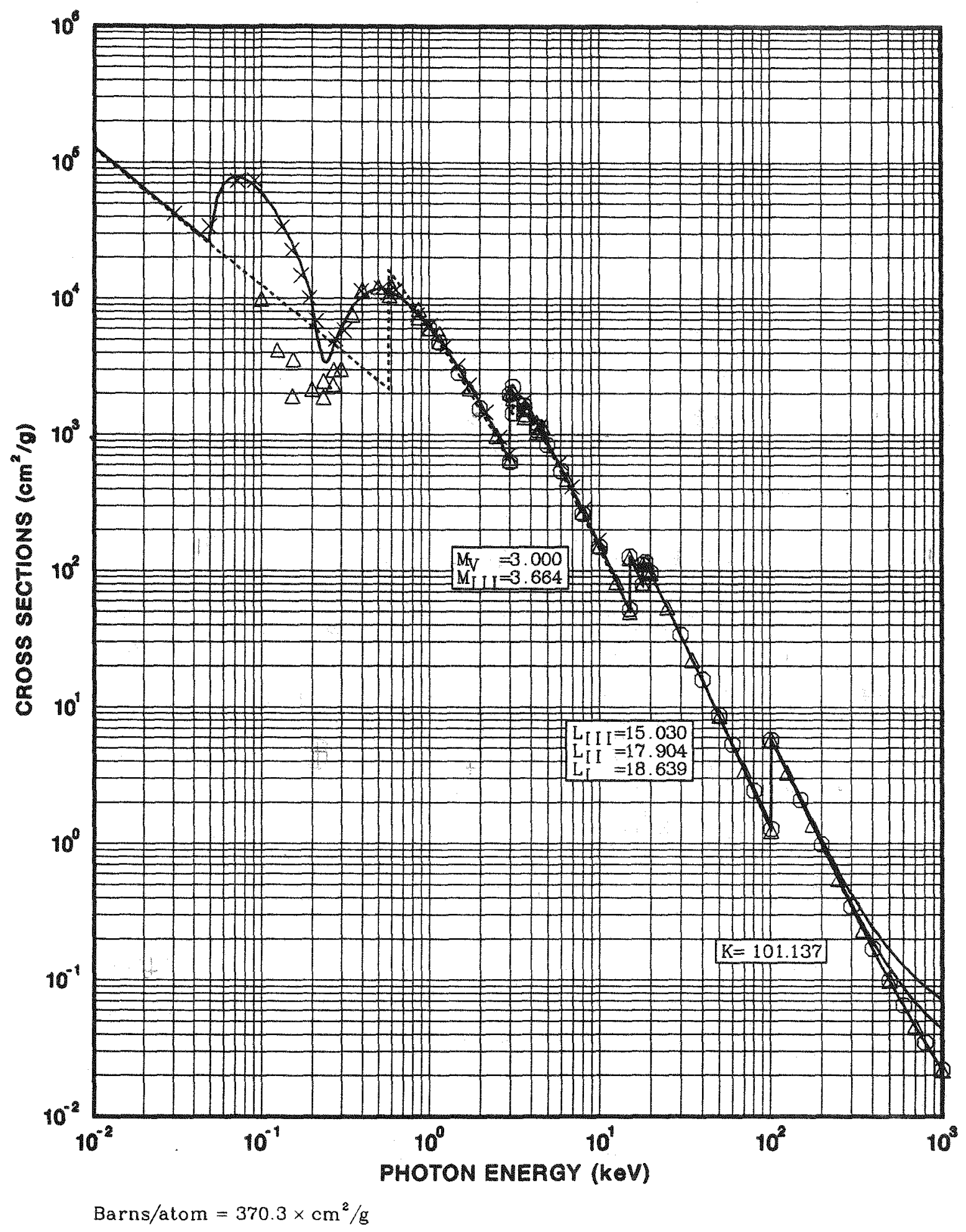


RADIUM 88

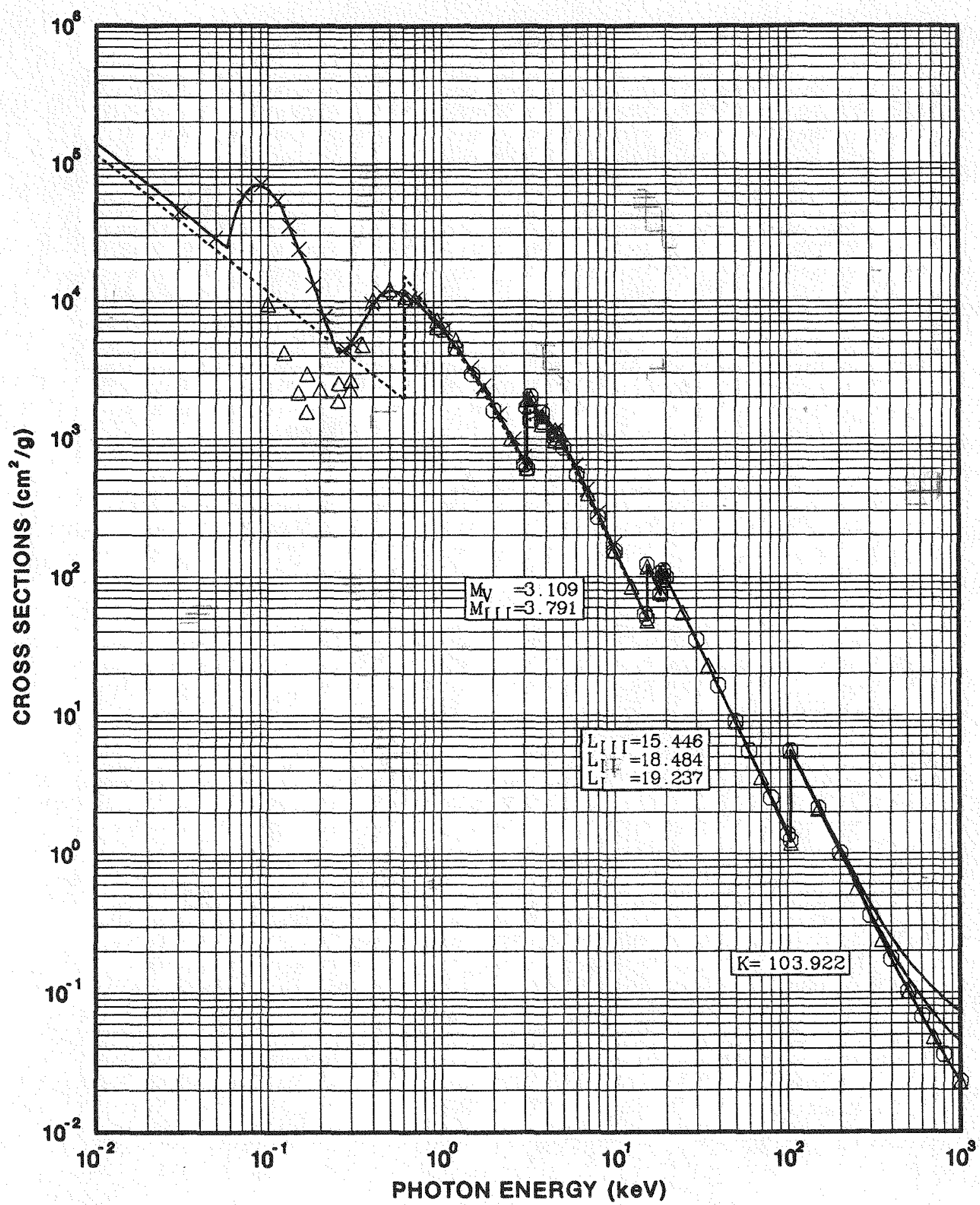

Barns/atom $=375.3 \times \mathrm{cm}^{2} / \mathrm{g}$ 


\section{ACTINIUM 89}

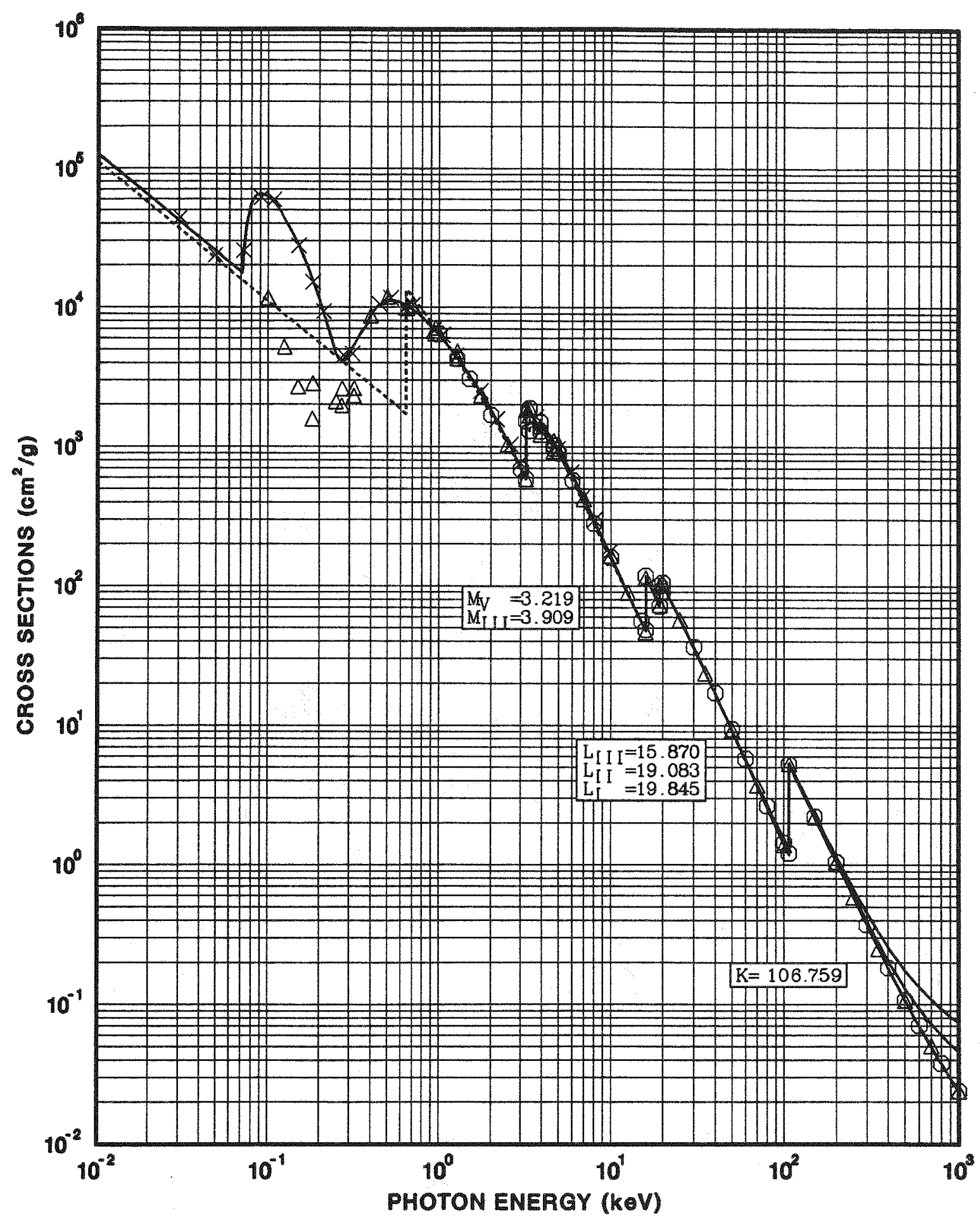

Barns/atom $=377.0 \times \mathrm{cm}^{2} / \mathrm{g}$ 
THORIUM 90

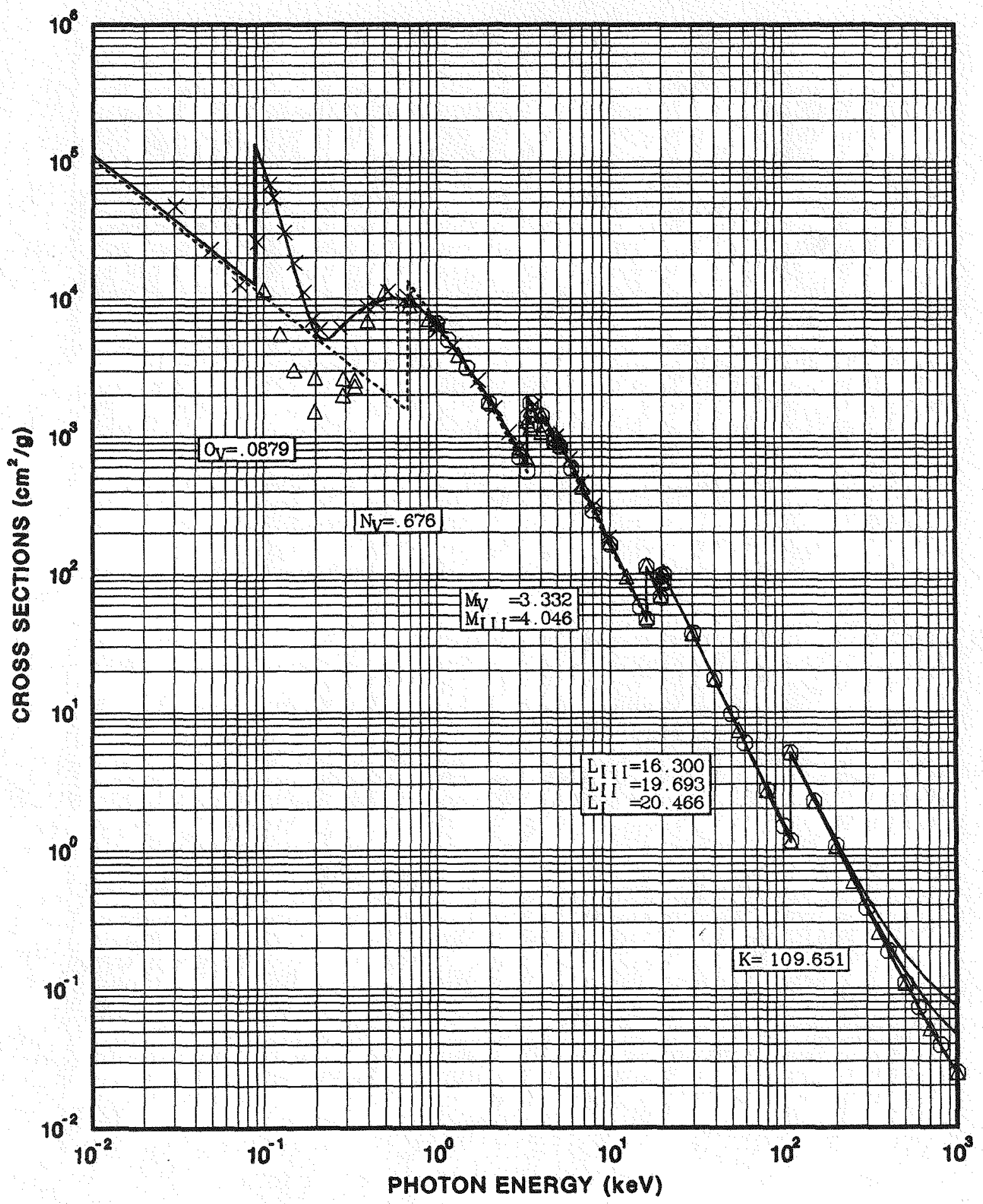

Barns/atom $=385.3 \times \mathrm{cm}^{2} / \mathrm{g}$ 
PROTACTINIUM 91

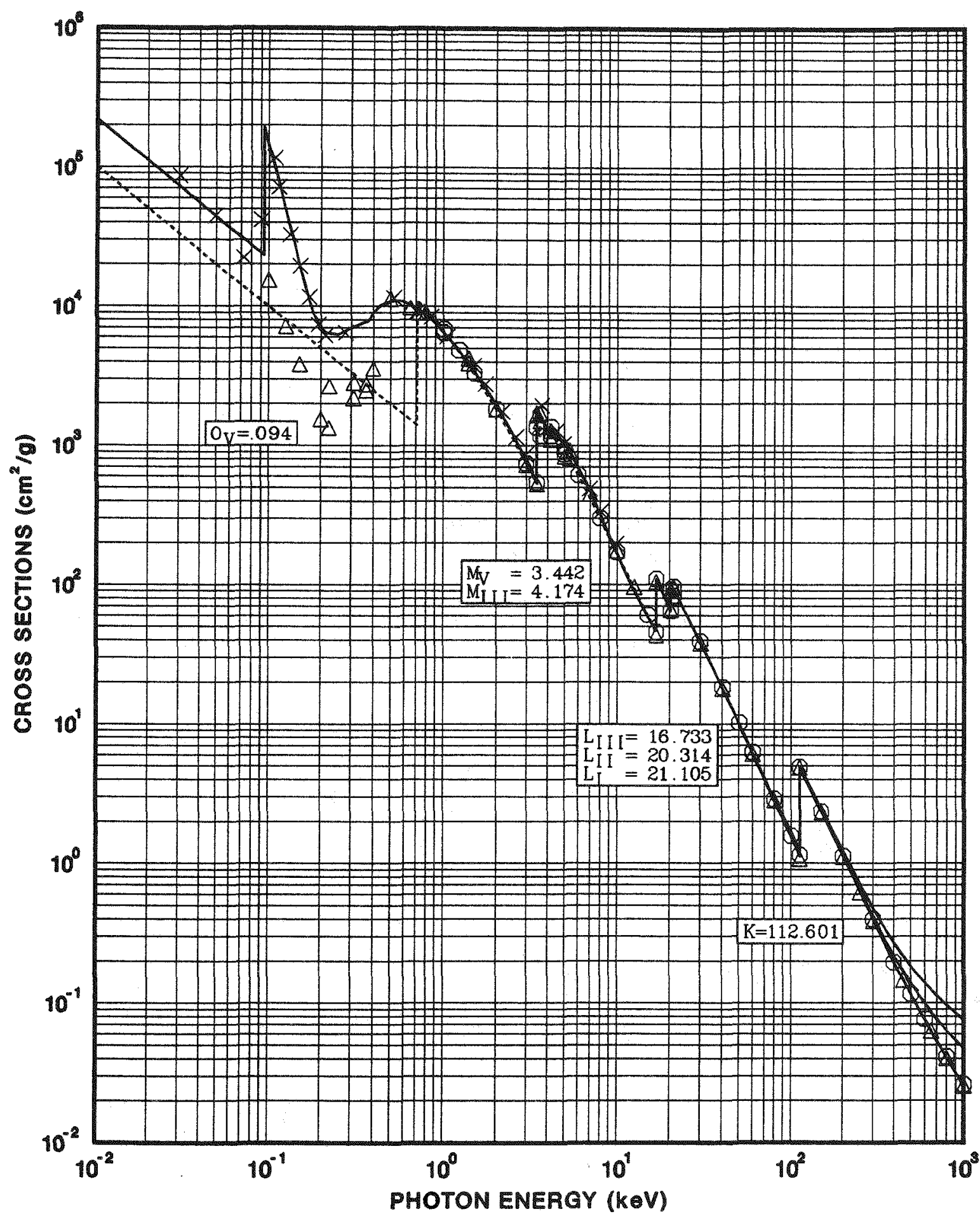

Barns/atom $=383.7 \times \mathrm{cm}^{2} / \mathrm{g}$ 
URANIUM 92

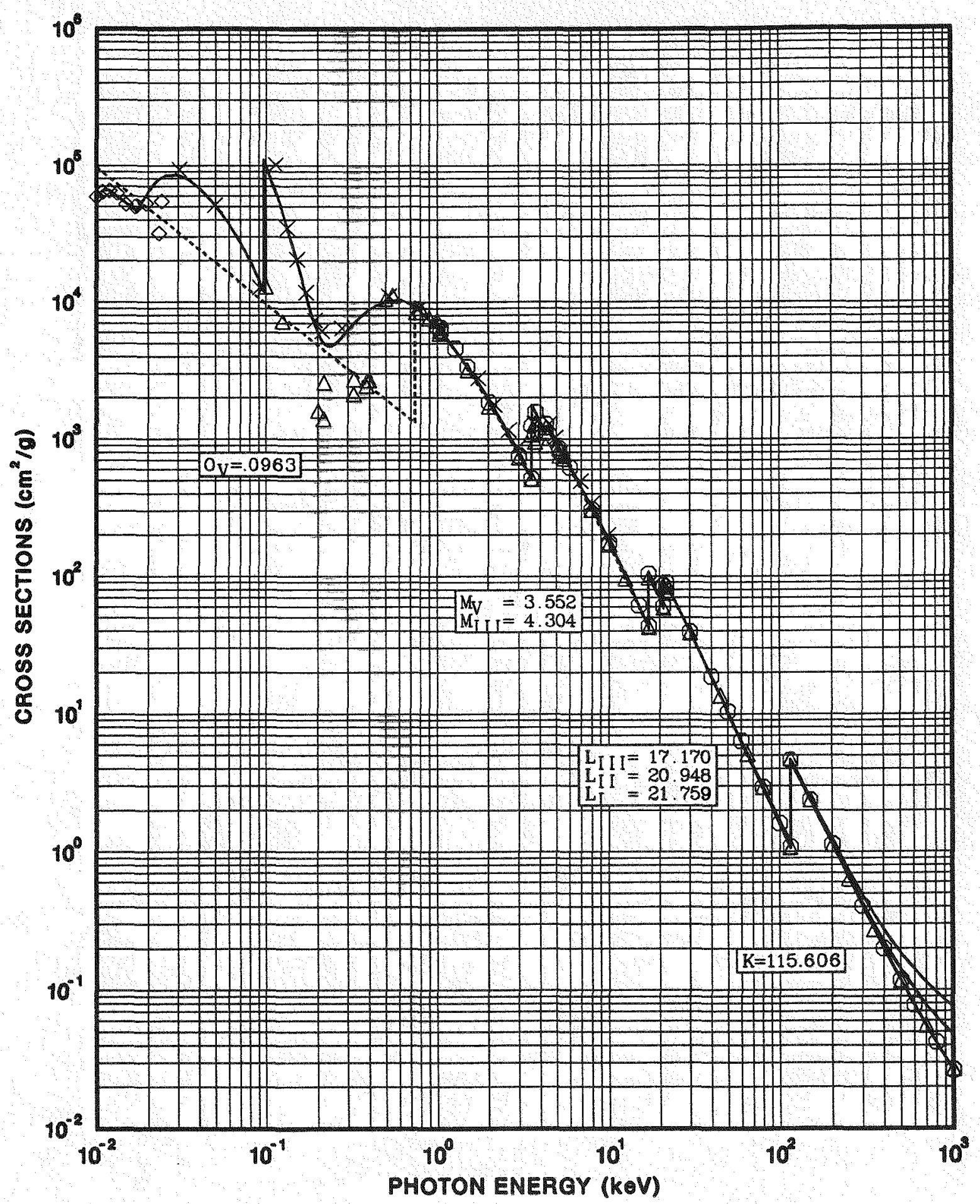

Barns/atom $=395.3 \times \mathrm{cm}^{2} / \mathrm{g}$ 


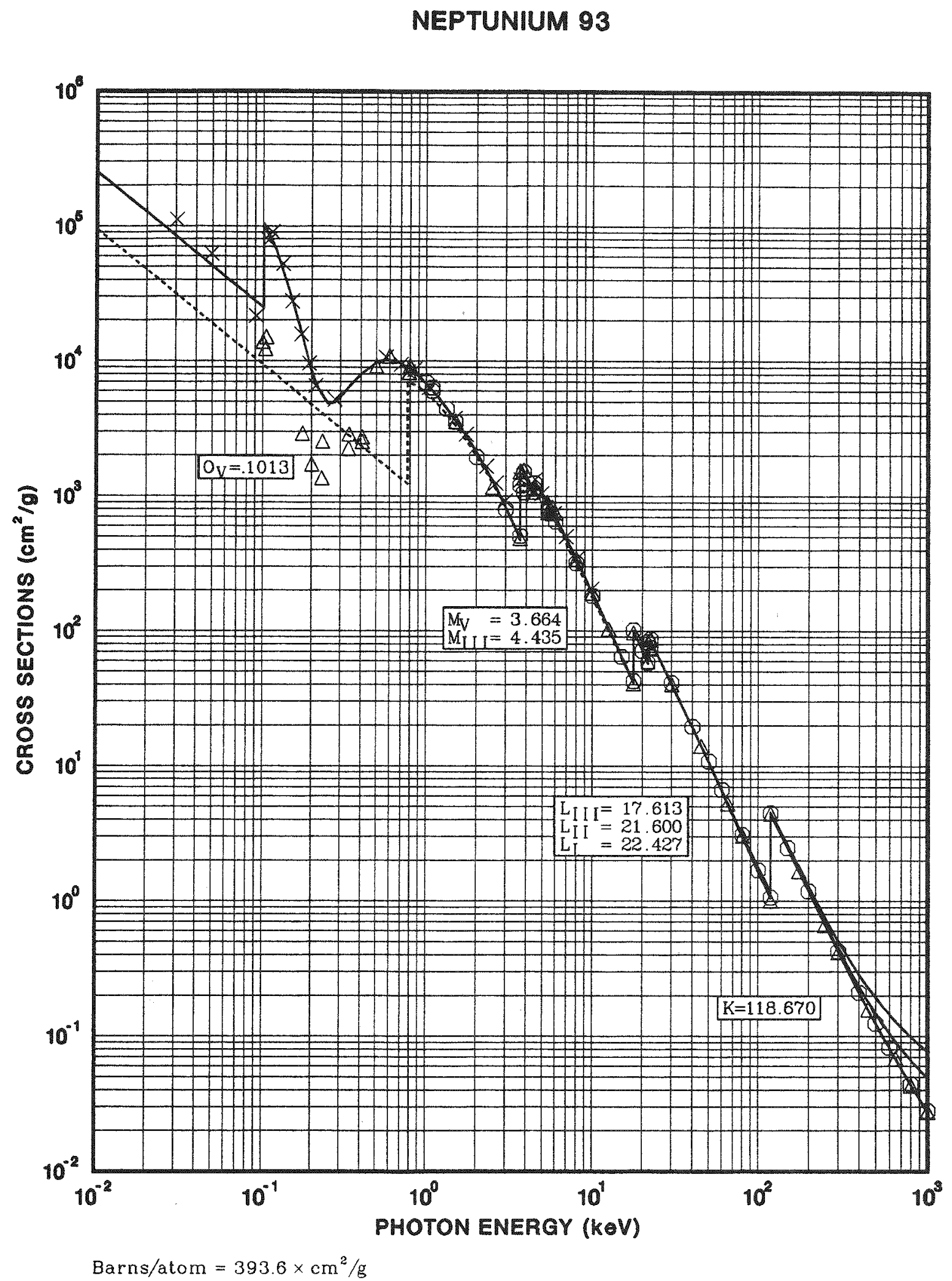




\section{PLUTONIUM 94}

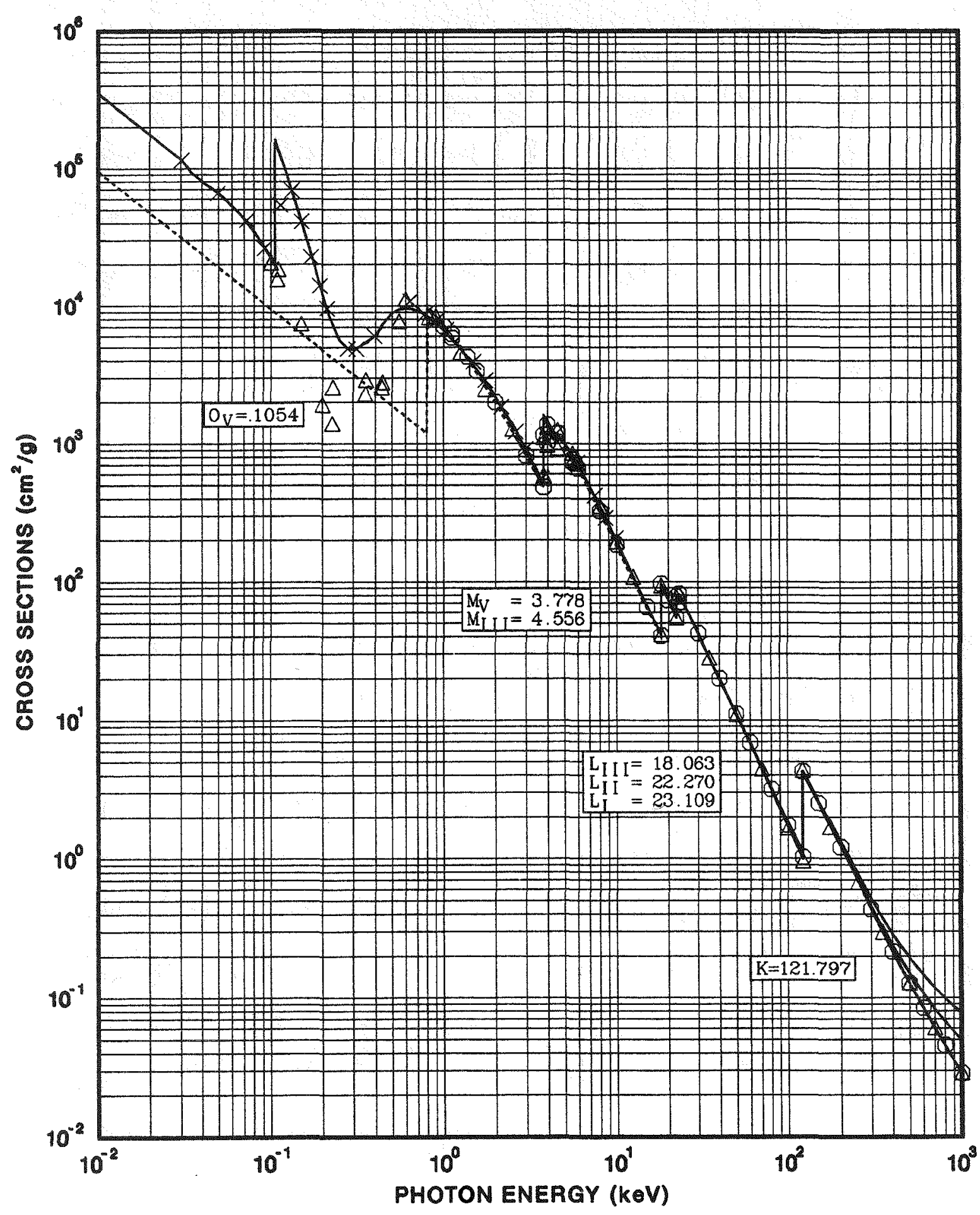

Barns/atom $=405.2 \times \mathrm{cm}^{2} / \mathrm{g}$ 


\section{AMERICIUM 95}

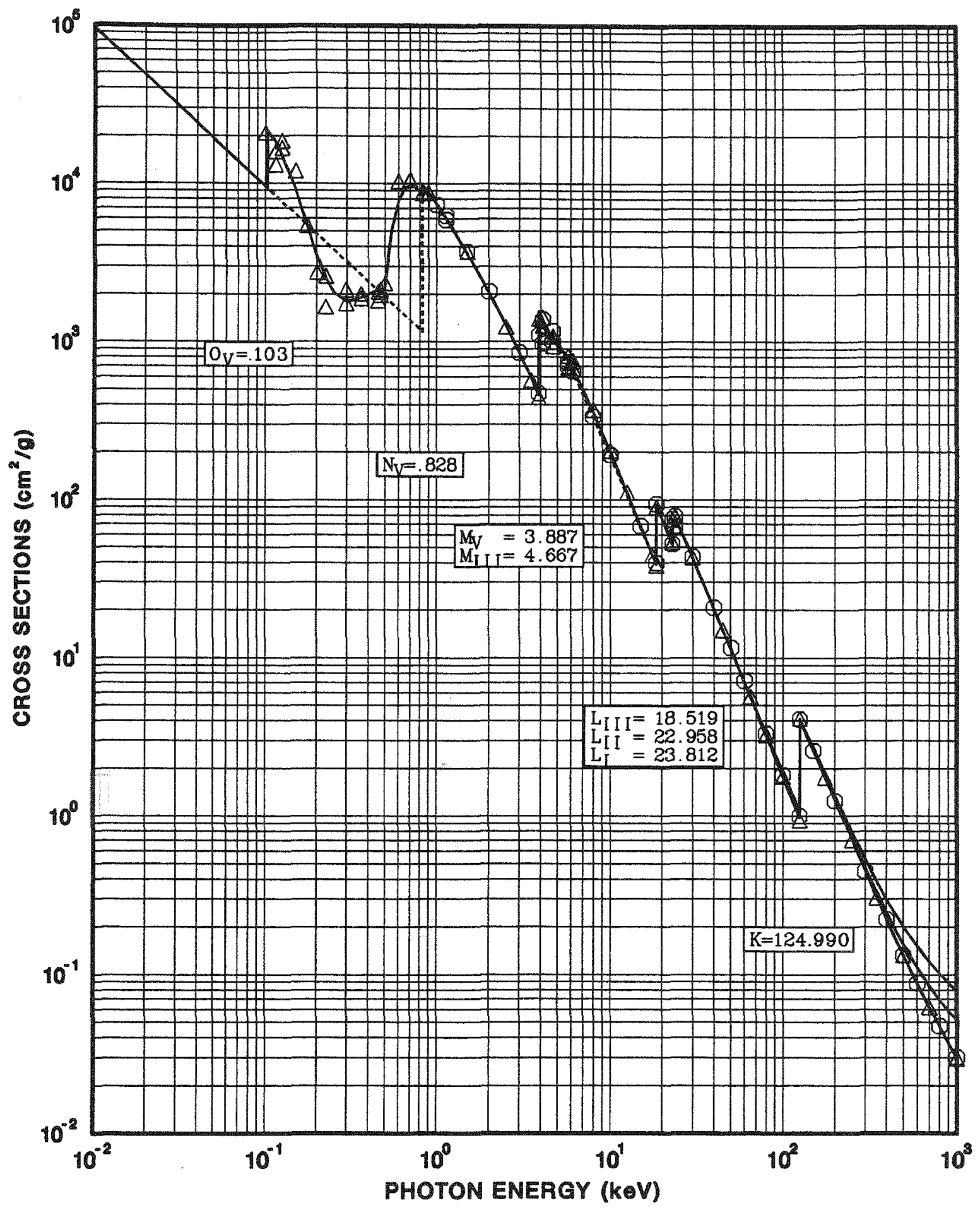

Barns/atom $=403.5 \times \mathrm{cm}^{2} / \mathrm{g}$ 
CURIUM 96

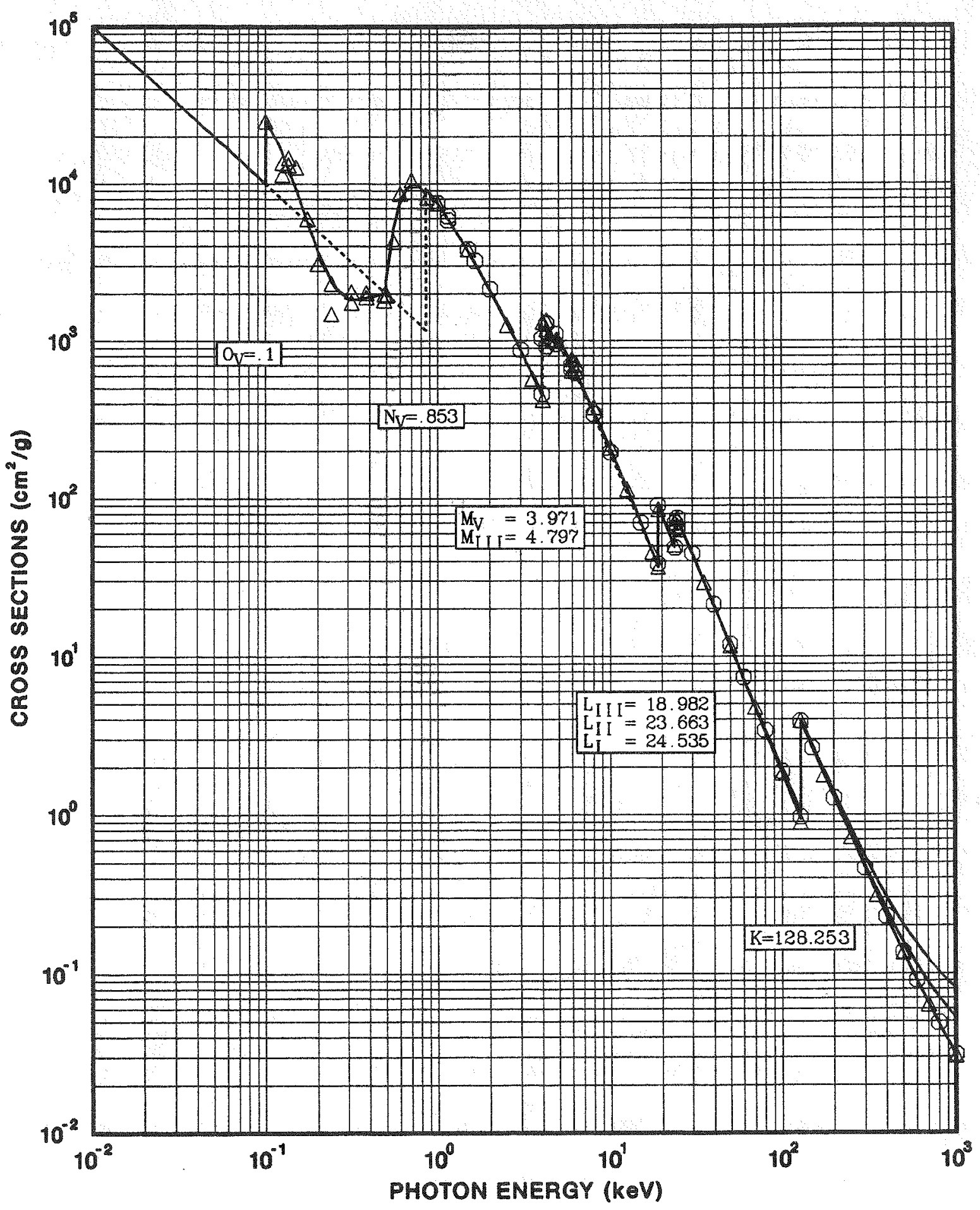

Barns $/$ atom $=410.2 \times \mathrm{cm}^{2} / \mathrm{g}$ 
BERKELIUM 97

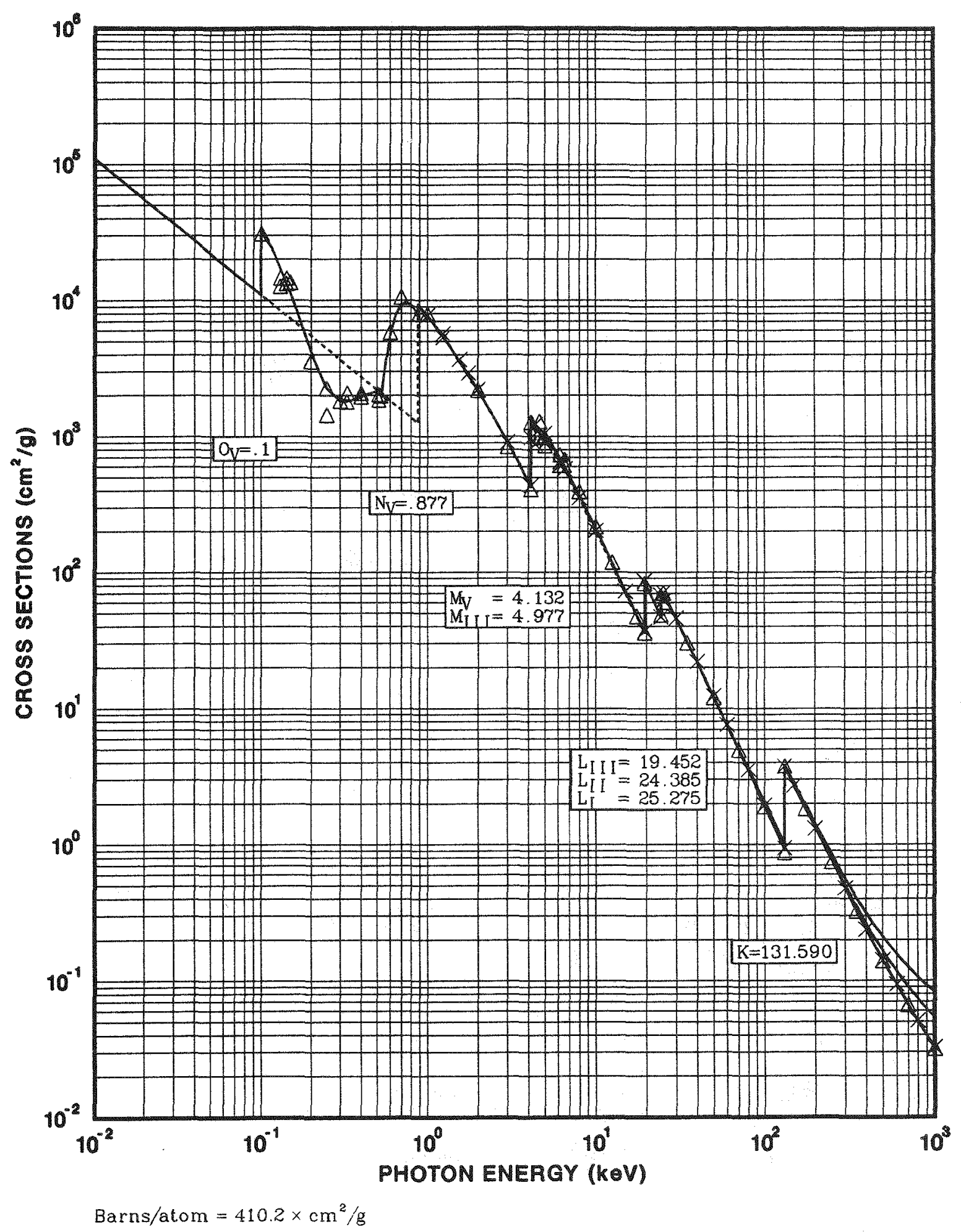




\section{CALIFORNIUM 98}

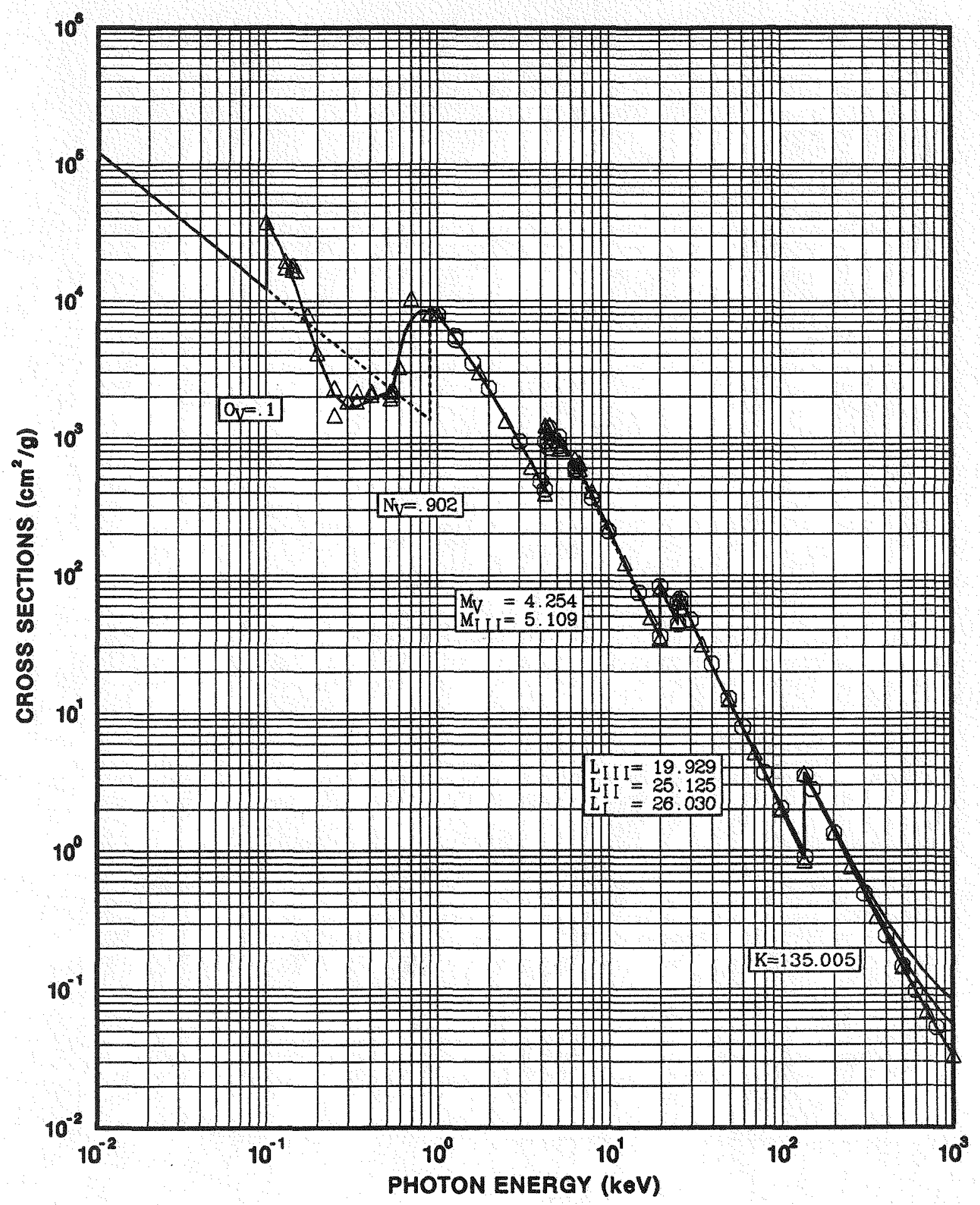

Barns/atom $=416.8 \times \mathrm{cm}^{2} / \mathrm{g}$ 


\section{EINSTEINIUM 99}

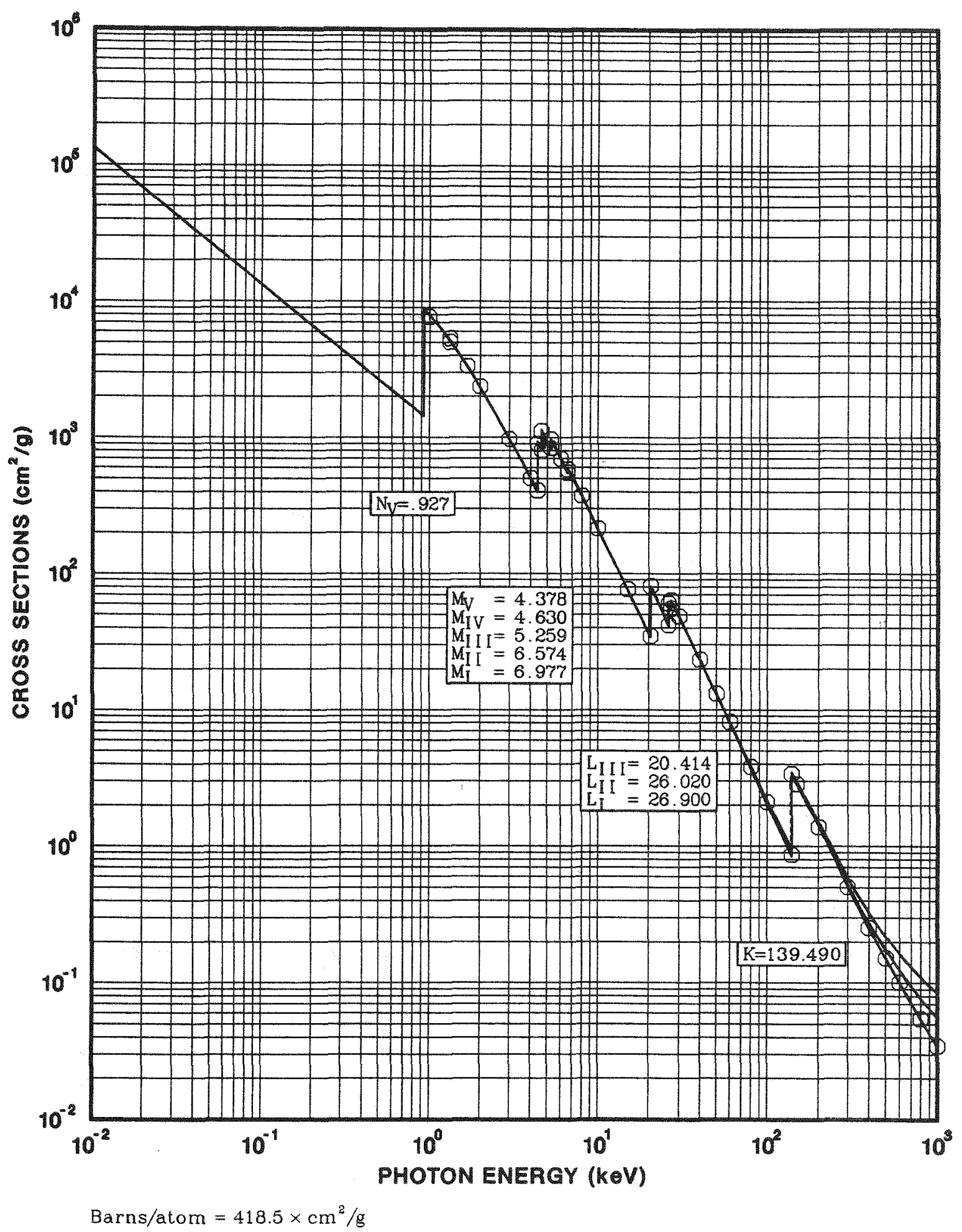


FERMIUM 100

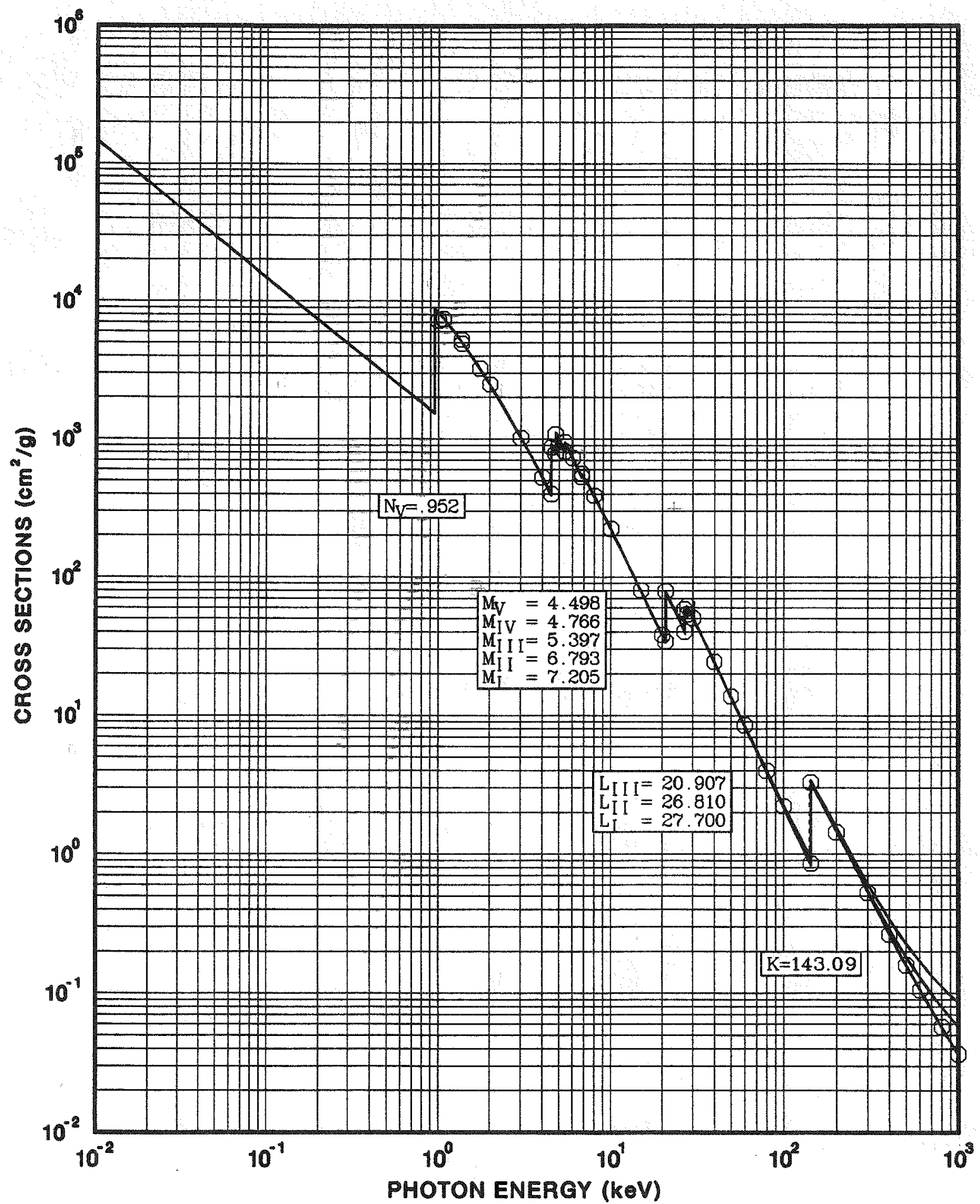

Barns/atom $=426.8 \times \mathrm{cm}^{2} / \mathrm{g}$ 


\section{References}

${ }^{1}$ F. Biggs and R. Lighthill, Analytical Approximations for X-Ray Cross Sections II, SC-RR-71 0507 (Albuquerque, NM: Sandia National Laboratories, December 1971).

${ }^{2}$ F. Biggs and R. Lighthill, Analytical Approximations for X-Ray Cross Sections, SC-RR-66-452 (Albuquerque, NM: Sandia National Laboratories, February 1967).

${ }^{3}$ R. C. Weast, Ed-in-Chief, CRC Handbook of Chemistry and Physics, 67th Ed (Boca Raton, FL: CRC Press, Inc., 1986-1987).

${ }^{4}$ D. Evans, Handbuch der Physik, Vol XXXIV (Berlin: Springer-Verlag, 1958) p 218.

${ }^{5}$ B. L. Henke, P. Lee, T. J. Tanaka, R. L. Shimabukuro, and B. K. Fujikawa, "Low-Energy X-Ray Interaction Coefficients: Photoabsorption, Scattering, and Reflection," Atomic Data and Nuclear Data Tables, Vol 27, Number 1 (New York, NY: Academic Press, January 1982).

${ }^{6}$ E. F. Plechaty, D. E. Cullen, and R. J. Howerton, Tables and Graphs of Photon-Interaction Cross Sections From $0.1 \mathrm{keV}$ to $100 \mathrm{MeV}$ Derived from the LLL EvaluatedNuclear-Data Library, UCRL-50400, Vol 6, Rev 2 (Livermore, CA: Lawrence Livermore National Laboratory, December 7, 1978).
${ }^{7}$ R. W. Roussin, J. R. Knight, J. H. Hubbell, and R. J. Howerton, Description of the DCL-99/HUGO Package of Photon Interaction Data in ENDF/B-V Format, ORNL/RSIC-46 (ENDF-335) (Oak Ridge, TN: Oak Ridge National Laboratory, December 1983).

${ }^{8}$ H. J. Hagemann, W. Gudat, and C. Kunz, Optical Constants from the Far Infrared to the X-Ray Region: $M g$, $\mathrm{Al}, \mathrm{Cu}, \mathrm{Ag}, \mathrm{Au}, \mathrm{Bi}, \mathrm{C}$, and $\mathrm{Al}_{2} \mathrm{O}_{3}, \mathrm{DESY}$ SR-74/7 (W. Germany, Deutsches Elektronen-Synchrotron DESY, May 1974).

${ }^{9}$ J. H. Weaver, C. Krafka, D. W. Lynch, and E. E. Koch, Optical Properties of Metals, Pt. I: The Transition Metals ( $0.1 \leq h \nu \leq 500 \mathrm{eV})$, Physik Daten, ISSN 0344-8401 NR.18-1 (Federal Republic of Germany: Faqchinformationszentrum, 1981).

${ }^{10}$ J. H. Weaver, C. Krafka, D. W. Lynch, and E. E. Koch, Optical Properties of Metals, Pt. II: Noble Metals, Aluminum, Scandium, Yttrium, the Lanthanides and the Actinides $(0.1 \leq h \nu \leq 500 \mathrm{eV})$, Physik Daten, ISSN 03448401 NR. 18-2 (Federal Republic of Germany: Fachinformationszentrum, 1981).

${ }^{11}$ D. Y. Smith, E. Shiles, and M. Inokuti, The Optical Properties and Complex Dielectric Function of Metallic Aluminum Form 0.04 to $10^{4} \mathrm{eV}$, ANL-83-24 (Chicago, IL: Argonne National Laboratory, March 1983). 


\section{DISTRIBUTION:}

Field Command (6)

Defense Nuclear Agency

Attn: Capt. R. B. Daniel, TTTT

W. J. Summa, TDTT

Maj. J. B. Ashbaugh, TTUT

Maj. C. Smythe, TTUT

Maj. A. E. Exten, NVTO

Commander J. Gogolin, TTTT

Kirtland AFB, NM 87115-5000

Lawrence Berkeley Lab (2)

Attn: J. Davis

B. L. Henke

1 Cyclotron Rd., MS 80-101

Berkeley, CA 94720

National Bureau of Standards

Attn: J. H. Hubbell

Bldg. Radiation Physics

Gaithersburg, MD 20899

Lawrence Livermore National Laboratory

Attn: J. Schofield, L-296

Livermore, CA 94550-0622

Los Alamos National Laboratory (3)

Attn: G. Doolan

J. E. Morel, MS B226

C. McInteer, Group NL

Los Alamos, NM 87545

S-Cubed

Attn: C. E. Wilson

PO Box 1620

LaJolla, CA 92038

Kaman Sciences Corporation (2)

Attn: D. Steele R. Zambola

PO Box 7463

Colorado Springs, CO 80933

Lockheed Missiles and Space Co.

Attn: J. Cayot, MS 0/8163

PO Box 504

Sunnyvale, CA 94086
SPIRE Corp.

Attn: J. Johnson

Patriots Park

Bedford, MA 01730

Lockheed Palo Alto Research Laboratory

Attn: S. Salisbury, Dept. 91-10, Bldg. 203

3251 Hanover St.

Palo Alto, CA 94304

SAI

Attn: K. Sites

PO Box 19057

Las Vegas, NV 89119-0057

Schlumberger Well Services

Attn: D. C. McKeon

PO Box 2175

5000 Gulf Freeway

Houston, TX 77252-2175

Argonne National Laboratories

Attn: D. Y. Smith, Bldg. 223

Materials Science Tech Division

Argonne IL 60439

Lockheed Missiles and Space Co.

Attn: R. Hess

PO Box 504

Sunnyvale, CA 94086

Ktech Corp.

Attn: F. Smith

901 Pennsylvania NE

Albuquerque, NM 87110

Applied Physics, Inc. (3)

Attn: R. L. Knight

R. W. Holland

C. Lee

5353 Wyoming NE, Ste. 3

Albuquerque, NM 87109

1113 M. A. Butler

1126 M. E. Riley 
DISTRIBUTION (Continued):

\begin{tabular}{|c|c|c|c|}
\hline 1151 & T. A. Green & 2531 & J. P. Anthes \\
\hline 1230 & J. E. Powell & 2531 & R. F. Carson \\
\hline 1231 & J. R. Lee & 2533 & W. J. Kass \\
\hline 1231 & J. A. Halbleib & 5151 & M. E. Senglaub \\
\hline 1231 & L. J. Lorence & 5152 & J. L. Duncan \\
\hline 1231 & T. W. Sanford & 5152 & F. F. Dean \\
\hline 1232 & W. Beezhold & $5160 \mathrm{~A}$ & J. F. Cuderman \\
\hline 1233 & L. M. Choate & 6400 & D. J. McCloskey \\
\hline 1234 & R. J. Leeper & 6418 & R. K. Cole \\
\hline 1234 & J. E. Bailey & 6420 & J. V. Walker \\
\hline 1234 & G. T. Baldwin & 6427 & M. Berman \\
\hline 1234 & D. L. Fehl & 6440 & D. A. Dahlgren \\
\hline 1235 & J. M. Hoffman & 6452 & J. W. Bryson \\
\hline 1261 & E. J. McGuire & 6454 & G. L. Cano \\
\hline 1265 & T. A. Mehlhorn & 6454 & J. G. Kelly \\
\hline 1265 & M. A. Sweeny & 6512 & P. J. McDaniel \\
\hline 1273 & M. K. Matzen & 7100 & C. D. Broyles \\
\hline 1273 & R. B. Spielman & 7110 & J. D. Plimpton \\
\hline 1422 & D. E. Amos & 7111 & J. R. Banister \\
\hline 1531 & S. L. Thompson & 7112 & C. R. Mehl \\
\hline 2300 & J. L. Wirth & 7112 & F. Biggs (50) \\
\hline 2320 & J. H. Renken & 7112 & A. J. Chabai \\
\hline 2321 & E. F. Hartman & 7112 & K. M. Glibert \\
\hline 2321 & A. Badruzzaman & 7112 & J. Harris \\
\hline 2321 & T. F. Calocci & 7112 & L. D. Kissel \\
\hline 2321 & C. Drumm & 7112 & R. E. Lighthill (25) \\
\hline 2321 & T. A. Haill & 7112 & M. A. Palmer \\
\hline 2321 & J. J. Hohlfelder & 7112 & T. J. Tanaka \\
\hline 2321 & D. C. Iwan & 7112 & D. R. Waymire \\
\hline 2321 & L. D. Posey & 7112 & A. Widman \\
\hline 2321 & D. M. Tanner & 7116 & W. H. Barrett \\
\hline 2322 & G. J. Scrivner & 7124 & P. L. Nelson \\
\hline 2322 & W. C. Fan & 7553 & M. E. Morris \\
\hline 2322 & S. B. Roeske & 8233 & J. M. Harris \\
\hline 2322 & C. N. Vittitoe & 9111 & T. S. Prevender \\
\hline 2334 & J. L. Wilcoxen & 9112 & S. A. Dupree \\
\hline 2342 & R. M. Delsner & 9122 & M. J. Forrestal \\
\hline 2361 & D. K. Yearout & 9231 & C. J. MacCallum \\
\hline 2363 & G. E. Clark & 9241 & J. M. Holovka \\
\hline 2500 & D. B. Hayes, Actg. & 8524 & P.W. Dean \\
\hline 2514 & L. L. Bonzon & 3141 & S. A. Landenberger (5) \\
\hline 2514 & R. F. LeBlanc & 3151 & W. I. Klein (3) \\
\hline 2515 & P. D. Wilcox & $3154-1$ & For DOE/OSTI (8) \\
\hline
\end{tabular}

